\title{
Spektroskopische Erfassung der Gastemperatur im Brennraum von Ottomotoren
}

\author{
DISSERTATION \\ zur Erlangung des mathematisch-naturwissenschaftlichen Doktorgrades \\ „Doctor rerum naturalium“ \\ der GeOrG-August-Universität Göttingen
}

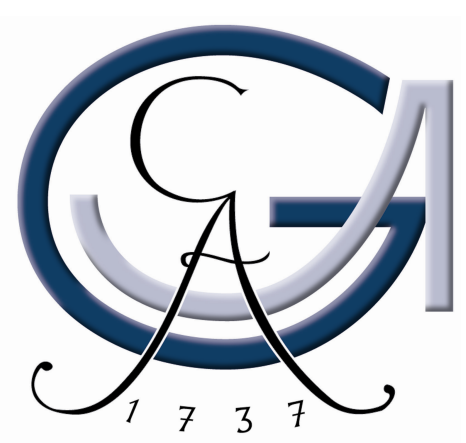

vorgelegt von:

RALF MÜLLER

(Diplom-Physiker)

aus

Alfeld/Leine

Göttingen 2009 
D7

Referent: Prof. Dr. Werner Lauterborn

Korreferent: Prof. Dr. Gerd Marowsky

Tag der mündlichen Prüfung: 17.12.2009 


\section{Inhaltsverzeichnis}

1 Einleitung 1

2 Motivation 3

3 Grundlagen ottomotorischer Verbrennung 7

3.1 Ottomotorische Brennverfahren . . . . . . . . . . . . 7

3.1.1 Gemischbildung . . . . . . . . . . . . . . . 7

3.1.2 Zündung und Verbrennung . . . . . . . . . . . . . . . . 12

3.1.3 Homogene Kompressionszündung . . . . . . . . . . . . . . . . 14

4 Wissenschaftliche Grundlagen des Messverfahrens $\quad 17$

4.1 Spektroskopische Verfahren in der Motordiagnostik . . . . . . . . . . 17

4.2 Laserinduzierte Fluoreszenz . . . . . . . . . . . . . . . . . . . . . . . . . . . 28

4.3 Emissionsspektroskopie von Molekülen . . . . . . . . . . . . 23

4.4 Reaktionskinetik der Verbrennung . . . . . . . . . . . . . . . . 25

4.5 Eigenschaften des Formaldehyds . . . . . . . . . . . . . . . . . 26

4.5.1 Verfahrenspezifische Eigenschaften . . . . . . . . . . 30

5 Technologische Grundlagen des Messverfahrens 33

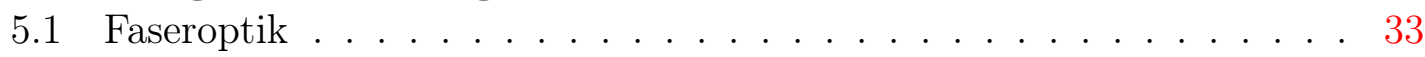

5.2 Laserstrahlhomogenisierung . . . . . . . . . . . . . . . . . 39

5.3 CCD-Kamera . . . . . . . . . . . . . . . . . . . 40

5.4 Spektrometer . . . . . . . . . . . . . . . . . . 44

5.5 Spektrale Filter . . . . . . . . . . . . . . . . . . 46

5.6 Optische Messverfahren an Motoren . . . . . . . . . . . . . . . . 47

5.7 Sensorsysteme für motorische Messungen . . . . . . . . . . . . . . . . 49

5.7 .1 Sensor 1. Generation . . . . . . . . . . . . . . . 51

5.7 .2 Sensor 2. Generation . . . . . . . . . . . . . . 53

6 Untersuchungen des Messverfahrens 55

6.1 Experimenteller Aufbau . . . . . . . . . . . . . . . . . 55

6.2 Spektroskopische Voruntersuchungen . . . . . . . . . . . . . 58

6.2.1 Fluoreszenzanregung von Formaldehyd . . . . . . . . . . 58

6.2.2 Fluoreszenzlebensdauermessungen . . . . . . . . . . . . . 60

6.2.3 Thermalisierung von Formaldehyd . . . . . . . . . . . . . . 61 
6.2.4 Fluoreszenzstabilität von Formaldehyd . . . . . . . . . . . 63

6.3 Baugruppenspezifische Einflussgrößen . . . . . . . . . . . . . . . . . 64

6.4 Statische Untersuchungen des Verfahrens . . . . . . . . . . . . . . . 65

6.4.1 Präparation der untersuchten Gemische . . . . . . . . . . . 65

6.4.2 Auswertung der Messergebnisse . . . . . . . . . . . . . 67

6.4.3 Statische Messungen an der Druckkammer . . . . . . . . . . 72

6.4.4 Sensormessungen an der Druckkammer . . . . . . . . . . . 76

6.5 Sensormessungen am Motor . . . . . . . . . . . . . . . . . 81

6.5.1 Physikalische Größen des Motors . . . . . . . . . . . . 81

6.5.2 Stöchiometrie der Gemische . . . . . . . . . . . . . . . 83

6.5.3 Temperaturbestimmung nach Arrhenius . . . . . . . . . . . 85

6.5.4 Abschließende Betrachtung . . . . . . . . . . . . . . 86

$\begin{array}{lll}7 & \text { Zusammenfassung } & 89\end{array}$

8 Ausblick 93

A Technische Zeichnungen des Sensorsytems 95

A.1 Konstruktionszeichungen des Sensors der 1. Generation . . . . . . . . 95

A.2 Konstruktionszeichungen des Sensors der 2. Generation . . . . . . . . 95

$\begin{array}{ll}\text { B Quellcode der Auswertung } & 109\end{array}$

$\begin{array}{ll}\text { Abbildungsverzeichnis } & 113\end{array}$

$\begin{array}{ll}\text { Literaturverzeichnis } & 115\end{array}$ 


\section{Kapitel 1}

\section{Einleitung}

Als Gottlieb Daimler zusammen mit Wilhelm Maybach 1883 den ersten benzinbetriebenen Verbrennungsmotor nach dem Prinzip von Nikolaus August Otto als Patent angemeldet hatte, lösten sie damit eine folgenreichende Entwicklung aus. Der erste Einzylinder-Viertaktmotor war nun so kompakt, dass der Geburtsstunde des Automobils nichts mehr im Wege stand. Diese Erfindung ermöglicht der Menschheit bis heute eine große, individuelle Bewegungsfreiheit. Über einen langen Entwicklungszeitraum ist diese Technologie zu einem festen Bestandteil des Alltags herangewachsen und in der heutigen Gesellschaft zu einem wichtigen Faktor des Privatlebens und der Wirtschaft geworden. Der von Carl Benz entwickelte und damals von der Öffentlichkeit als „Wagen ohne Pferde“ belächelte Patent-Motorwagen-Nummer 1 hat mit den heutigen Automobilen keine Ähnlichkeit mehr. Die Technik hat immense Fortschritte gemacht und den antriebswirksamen Prozess immer wieder optimiert. Trotz der heute zur Verfügung stehenden Erkenntnisse in der Motorentechnologie existiert weiterhin das Problem der Umweltbelastung durch Schadstoffe, die bei der Verbrennung von fossilen Kraftstoffen entstehen. Des Weiteren sind die Ressourcen zur Gewinnung des Kraftstoffes nur begrenzt verfügbar. Ein stetig ansteigender globaler Bedarf an Kraftfahrzeugen erhöht somit im gleichen Maße die ökologischen Folgen und minimiert den Rohstoffbestand. Die Tatsache, dass sich diese bewährte Antriebstechnologie gut in die Gesellschaft integrieren lässt und der stark wachsende Wunsch nach Flexibilität, verleiht ihr auch weiterhin eine Zukunft. Um der Problematik entgegen zu wirken, werden Umweltgesetze erlassen, die immer strengere Grenzwerte für Schadstoffemissionen beinhalten. Die Motorenentwicklung hat somit das klare Ziel, diese Anforderungen zu erfüllen und den Kraftstoffverbrauch von Verbrennungsmotoren weiter zu reduzieren. Diese Erhöhung des Wirkungsgrades und die damit einhergehende verminderte Schadstoffentstehung kann nur Erfolg haben, wenn die innermotorischen, physikalischen und chemischen Vorgänge genau bekannt und kontrollierbar sind. Um die Verbrennung verstehen und darauf Einfluss nehmen zu können, müssen schon sehr viel früher die Untersuchungen einsetzen und zwar in der Gemischbildung. Sie wirkt sich entscheidend auf die Verbrennungsqualität aus. Neben der Frage, wie die ideale Verteilung des Kraftstoff-Luft-Gemisches im Brennraum aussieht, hat sich der Fokus der Motorenforschung auf eine andere physikalische Größe gerichtet, nämlich auf die Temperatur des Gemisches. 
Die anfangs als Störfaktor wirkende Selbstzündung wird in neueren Konzepten wie dem sogenannten HCCI-Motor (engl. „Homogeneous Charge Compression Ignition“, Homogene Kompressionszündung) nutzbar gemacht und soll als kontrollierbarer Initiator der technischen Verbrennung dienen. Gerade die Erfoschung dieses Konzepts offenbart den starken Einfluss der Temperatur auf die ablaufenden chemischen Reaktionen. Somit ist die Kenntnis dieser thermodynamischen Größe ein essentieller Bestandteil, wenn man diesen physikalisch-chemischen Vorgang technisch nutzen möchte. Nun ist die Verbrennungskraftmaschine ein komplexes und dynamisches System, bei dem im Laborversuch nur schwer Untersuchungen unter realen Bedingungen durchführbar sind. Eine geeignete Methode bietet die laserunterstützte Spektroskopie, die sich schon oft in der Motordiagnostik bewährt hat.

Diese Arbeit beinhaltet die Erfassung der Temperatur in motorischen Systemen. Mit Hilfe der berührungslosen optischen Messtechnik ist ein minimal-invasives Sensorsystem entwickelt worden, welches die thermodynamischen Eigenschaften eines Motors im geringsten Maße beeinflusst. Zuvor fanden statische Messungen an einer Druckkammer statt, um motorische Bedingungen zu generieren. Das Prinzip beruht auf der Zwei-Linien-Thermometrie, bei der durch laserinduzierter Fluoreszenz ein Tracermolekül die Temperaturinformation liefert. Es fanden spektroskopische Untersuchungen von Formaldehyd statt, um die molekülspezifischen Eigenschaften auf die Eignung für das Messverfahren zu evaluieren. Im nächsten Schritt entstand ein anwendungsangepasster Ansatz des Temperaturmessverfahrens durch die Entwicklung eines Sensorsystems. Dabei sind zwei technische Umsetzungen auf die motorische Applikation realisiert worden. Die innerhalb der Testmessungen des ersten Sensorsystems gemachten Erkenntnisse wurden in die Konstruktion des zweiten Sensors einbezogen. Die vorgestellten Resultate aus den statischen Messungen beider Sensoren unterstrichen die Anwendbarkeit auf motorische Systeme. Mit Hilfe des zweiten entwickelten Sensorsystems fanden Messungen am Motorprüfstand des Laser-Laboratoriums Göttingen statt. Die dabei gewonnenen Ergebnisse und Erkenntnisse schließen diese Arbeit ab. 


\section{Kapitel 2}

\section{Motivation}

Ein in der Gesellschaft wichtiger Aspekt ist der Wunsch nach individueller Mobilität. Diese wichtige und nicht mehr wegzudenkende Größe kann zur Zeit von den Verbrennungskraftmaschinen am besten realisiert werden. Diese bereits alltägliche Technik, welche in den Automobilen zu finden ist, basiert auf Kraftstoffe in flüssiger Form, die sich gut transportieren lassen und somit der Verbrenungskraftmaschine ihre Mobilität verleiht. Dem Verlangen nach räumlicher Flexibilität steht die hohe ökologische Belastung durch Schadstoffe gegenüber, die durch die Kraftstoffverbrennung hervorgerufen wird. Des Weiteren sind die Ressourcen, die zur Gewinnung von Kraftstoff herangezogen werden, nur begrenzt verfügbar. Aufgrund des global zunehmenden Bedarfs an Automobilen und der damit steigenden Umweltbelastungen, sowie schwindenden Rohstoffen, wurden gesetzliche Rahmenrichtlinien geschaffen, um diese gegensätzliche Entwicklung einzudämmen. Innerhalb der EU wird dies zum Beispiel durch die sogenannte Euro-Norm durchgesetzt, die 1992 in Kraft getreten ist und Grenzwerte für die Schadstoffemission vorgibt, deren Richtlinien immer strengere Grenzwerte erhebt (siehe Abb.2.1). In der Abgasnorm wird ein stetiges Absinken vor allem des schädlichen Kohlenmonoxids (CO) diverser Kohlenstoffverbindungen ( $\mathrm{HC}$, engl. hydrocarbons) und von Stickstoffoxiden $\left(\mathrm{NO}_{\mathrm{x}}\right)$ gefordert. Aufgrund der mageren Verbrennung entsteht vor allem bei Dieselmotoren partikelförmiger Kohlenstoff. Daher ist für diesen Motortyp ein Grenzwert zur Partikelemission eingeführt worden. Da bei der ottomotorischen Verbrennung vor allem Kohlenwasserstoffverbindungen im Abgas zu finden sind, wird hier ein separater Grenzwert für diesen Schadstofftyp festgelegt. Ab der Euro-6-Norm werden erstmals sogenannte Nichtmethankohlenwasserstoffe (NMHC) in die Schadstoffliste aufgenommen.

Um diese und andere Umweltgesetze zu erfüllen, ist die Reduktion des Kraftstoffverbrauchs, was gleichzeitig eine Reduzierung der Schadstoffemission bewirkt, Ziel der Weiterentwicklung der heutigen Motorentechnologie. Neben den alternativen Antriebstechnologien, wie zum Beispiel in Erdgas- oder Elektrofahrzeuge realisiert, ist aufgrund der bestehenden Infrastruktur der Diesel- und Ottomotor weiterhin ein Antrieb mit Zukunft. Daher wird weltweit in den Forschungslaboren die Verbesserung des innermotorischen Brennverfahrens vorangetrieben und eine Erhöhung des Wirkungsgrades angestrebt. 


\section{Grenzwerte für Schadstoffemissionen von Diesel- und Benzin-PKW}

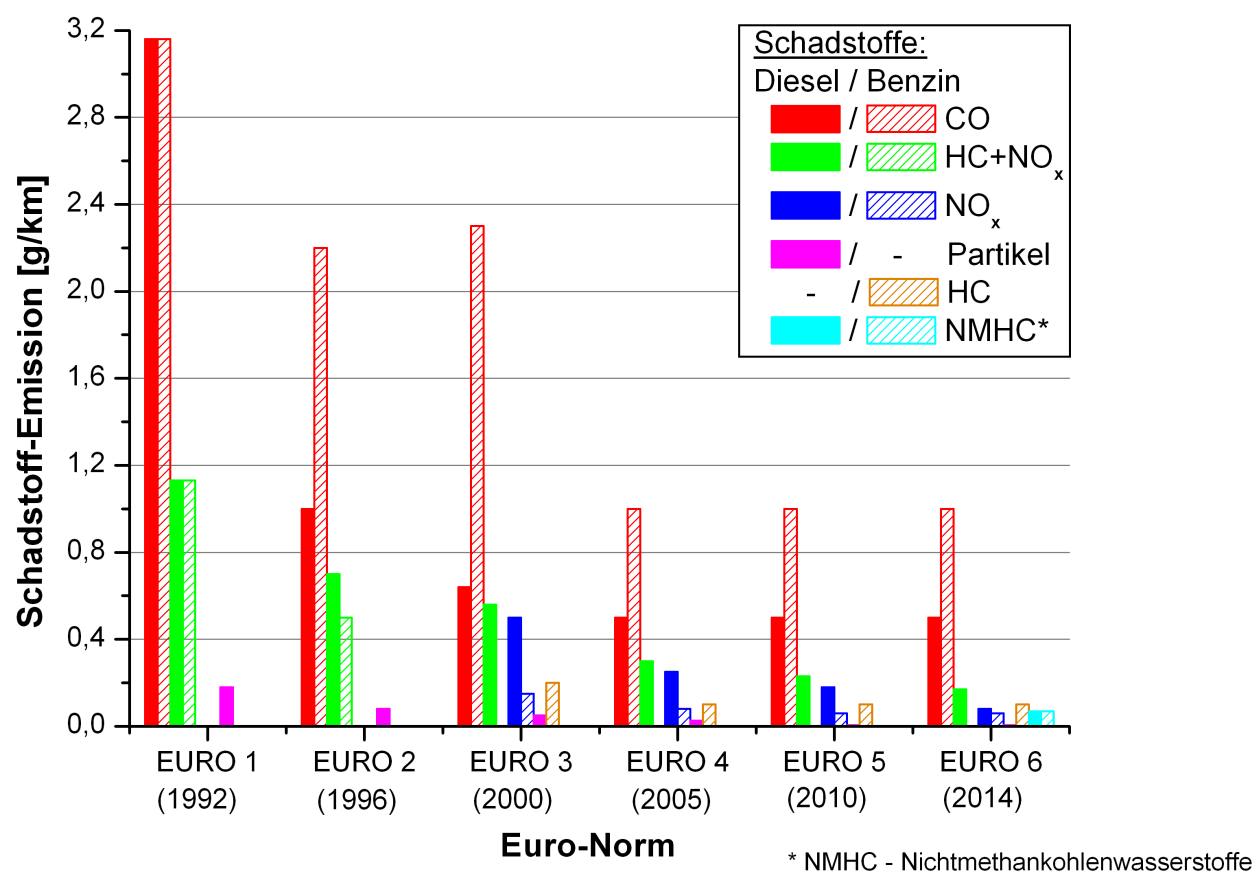

Abbildung 2.1: Entwicklung der EU-Abgasnorm für Diesel- und BenzinFahrzeuge. Es wird ein Grenzwert für die Konzentration von Kohlenwasserstoffen und Stickoxiden angegeben $\left(\mathrm{HC}+\mathrm{NO}_{\mathrm{x}}\right)$. Für $\mathrm{NO}_{\mathrm{x}}$ hingegen existiert eine Höchstgrenze. (Datenquelle: Umweltbundesamt)

Seit der Inbetriebnahme des ersten Ottomotors (1876) durch seinen Erfinder Nicolaus August Otto ([1],[2]) und später des Dieselmotors (1897) von Rudolf Diesel ([3],[4]) sind viele Erkenntnisse über diese Technologie gesammelt worden. Dieses über Jahrzehnte angesammelte Wissen ist in den heutigen modernen Otto- und Dieselfahrzeugen integriert. Beide Verfahren haben Vorteile, um eine weitere Verringerung sowohl des Kraftstoffverbrauchs als auch der Schadstoffemission zu erzielen. Daher arbeitet die Forschung seit einigen Jahren daran, diese beiden Technologien zu vereinigen. Kern dieser vielversprechenden Alternative stellt die sogenannte homogene Selbstzündung dar. Analog zum herkömmlichen Ottomotor wird ein homogenes Kraftstoff-Luft-Gemisch generiert und verdichtet. Bei ausreichender Kompressionsendtemperatur tritt hierbei eine Selbstzündung ein. Das als HCCI (engl. „Homogeneous Charge Compression Ignition") bekannte Verfahren hat einen niedrigen Kraftstoffverbrauch bei sehr geringer Schadstoff- und Rußemission. Diese Verminderung der Schadstoffentwicklung wird durch eine Vermischung des Gemisches mit Abgas erzielt. Dieser Sachverhalt hat den Effekt, dass eine aufwendige und kostspielige Abgasnachbehandlung wie bei den Diesel- und Ottomotoren nicht mehr benötigt wird. 
Ein weiterer Vorteil des HCCI-Motors ist seine hohe Effizienz.

Wichtig ist hierbei allerdings, dass die Verbrennung kontrolliert abläuft. Bei Teillast, bei der eine stöchiometrische Verbrennung stattfindet, funktioniert das HCCIKonzept recht gut. Schwierigkeiten stellen sich aber bei Variation der Motorgeschwindigkeit bzw. Lastwechsel, in der Kaltstartphase oder unter Vollast ein. Generell ist die extrem schnelle Verbrennung im HCCI-Motor ein großes Problem für die dynamische Steuerung der Selbstzündung. Herrscht im Zylinder eine hohe Temperatur, so kann es passieren, dass das Gemisch an vielen Orten gleichzeitig zündet. Bei einer vorzeitigen Verbrennung hat das heiße Gas zu langen Kontakt mit den Zylinderwänden und es treten zu hohe Wärmeverluste auf. Eine spätere Verbrennung führt dagegen zu Leistungsverlusten.

Gerade diese Problematik der Steuerung der Selbstzündung hat bewiesen, dass die Temperatur ein wichtiger Einflussparameter ist, da sie ein entscheidender Faktor bei der Einleitung der Verbrennung darstellt. Trotz der immensen Forschungstätigkeiten im Bereich der Motorenentwicklung ist es immer noch eine große Herausforderung, die innermotorische Verbrennung kontrolliert ablaufen zu lassen. Besonders die Entwicklung des HCCI-Brennverfahrens lenkte erneut den Fokus der Forschung auf die Temperatur des Kraftstoff-Luft-Gemisches vor der Zündung. In dieser Arbeit wird ein physikalisches Messverfahren entwickelt, welches sich dieser Herausforderung stellt. Diese auf laserinduzierter Fluoreszenz basierte und tracerunterstützte Zwei-Linien-Methode ermöglicht eine berührungslose Temperaturmessung. Das hierbei verwendete Tracermolekül Formaldehyd hat neben der Tatsache, dass es ein Zwischenprodukt der Verbrennung darstellt, den weiteren Vorteil, der guten Auflösung einzelner Vibrationsübergänge in dessen Fluoreszenzspektrum. Durch die Integration dieser laserunterstützen und berührungslosen Methode in ein faseroptisches Sensorsystem steht zudem eine minimal invasive Technologie zur Verfügung, die die thermodynamischen Eigenschaften von Serienmotoren so gut wie nicht verändert und damit eine Temperaturinformation unter realen Bedingungen liefert. Somit wird eine Möglichkeit vorgestellt, wie diese für die zukünftige Motorenentwicklung wichtige physikalische Größe an realen Motoren erfasst werden kann. 



\section{Kapitel 3}

\section{Grundlagen der ottomotorischen Verbrennung}

\subsection{Ottomotorische Brennverfahren}

Dieser Abschnitt gibt eine kurze Einführung in die Technologie der Verbrennungskraftmaschinen. Dabei handelt es sich hier um eine Kraftmaschine, innerhalb derer ein Wärmeprozess und ein Verbrennungsprozess gekoppelt ablaufen. Im Verlaufe dieses Prozesses wird die im Kraftstoff chemisch gebundene Energie durch Verbrennung in Form von thermischer Energie freigesetzt und in mechanische Energie umgewandelt. Die Kraftmaschine erzeugt eine vortriebswirksame Leistung. Diese Leistungsentfaltung durch Kolbenmaschinen findet in Form von Verbrennungsmotoren eine weite Verbreitung in der Gesellschaft. Die Vorteile der Verbrennungsmotoren liegen in der Möglichkeit des Betriebs mit flüssigem Kraftstoff hoher Energiedichte, welcher einen ortsunabhängigen Einsatz erlaubt. Weiterhin haben diese Maschinen einen relativ guten Wirkungsgrad und decken einen großen Leistungsbereich ab. Unter der Vielzahl von Motorarten soll hier hauptsächlich der Ottomotor beschrieben werden.

\subsubsection{Gemischbildung}

Der Ottomotor ist eine Kolbenmaschine mit innerer Verbrennung, welche sowohl mit einer äußeren als auch mit einer inneren Gemischbildung arbeitet. In älteren Motoren findet die äußere Gemischbildung durch den Vergaser statt, welcher den Kraftstoff streng genommen nur zerstäubt und in den vom Kolben erzeugten Ansaugluftstrom leitet. Mit Hilfe einer Drosselklappe wird bei diesem Prinzip die Abgabeleistung des Motors reguliert, indem die Luftzufuhr der benötigten Kraftstoffmenge über die Klappenöffnung angepasst wird. Aufgabe des Gemischbildungsverfahrens ist eine optimale Gemischverteilung für alle Lastbereiche zu erzeugen. Für die äußere Gemischbildung bedeutet dies eine Zuführung eines homogenen Gemisches aus Kraftstoff und Luft. Zunächst muss der flüssige Kraftstoff vollständig verdampfen und sich mit der Verbrennungsluft vermischen. Die Gemischbildung findet während des Ansaug- und Verdichtungstaktes statt. Diese Takte sind die ersten beiden Teil- 
takte des Arbeitszyklus eines modernen Viertakt-Motors, dessen Prinzip in Abbildung 3.1 verdeutlicht wird. Diese Motoren haben neben den eben Genannten noch den Arbeits- und den Ausstoßstakt.Während des Ansaugtaktes findet zunächst eine großräumige Mischung zwischen Luft und Kraftstoff statt, wobei kleine Tropfen (Durchmesser $\approx 20 \mu \mathrm{m}$ ) vollständig verdampfen. Im anschließenden Verdichtungstakt erfolgt eine intensivere Mischung, bei der auch große Tropfen (Durchmesser $\approx 200 \mu \mathrm{m}$ ) verdampfen. Um eine vollständige Verbrennung zu generieren, muss das Luftverhältnis des Kraftstoff-Luft-Gemisches gleich 1 sein. Das dem Motor tatsächlich zugeführte Verhältnis aus Luftmenge $m_{\text {Luft }}$ je Kraftstoffmenge $m_{K}$ zu der zur vollständigen Verbrennung dieser Kraftstoffmenge theoretisch benötigten Luftmenge

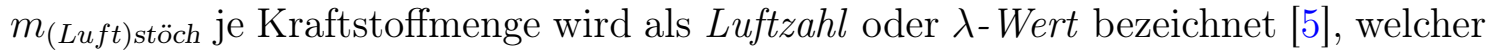
definiert ist als

$$
\lambda=\frac{m_{\text {Luft }} / m_{K}}{\left(m_{\text {Luft }} / m_{K}\right)_{\text {stöch }}}=\frac{m_{\text {Luft }} / m_{K}}{m_{\text {Luft }, \text { stöch }} / m_{K}}=\frac{m_{\text {Luft }}}{m_{\text {Luft,stöch }}}
$$

Bei Werten $\lambda<1$ liegt ein Luftmangel vor und es bleibt unverbrannter Kraftstoff übrig. Hier wird von einem ,fetten“ Gemisch gesprochen. Für $\lambda>1$ hingegen ist ein Luftüberschuss vorhanden und man bezeichnet dieses Gemisch als „mager“. Eine vollständige und somit stöchiometrische Verbrennung findet bei $\lambda=1$ statt. Der Zündbereich für Kraftstoff-Luft-Gemische befindet sich bei $0,4<\lambda<1,4$ [6]. Oberund unterhalb dieser Zündgrenzen ist ein Gemisch nicht zündfähig. Dieser Bereich muss bei der Gemischbildung erreicht werden. Innerhalb verschiedener Betriebszustände sorgen jedoch physiklalische Randbedingungen für Kraftstoffverluste und nehmen so Einfluss auf die Verbrennung. Diese Tatsache hat zur Folge, dass beim sogenannten Kaltstart mehr Kraftstoff eingespritzt wird, da aufgrund von niedrigen Wand- und Ansauglufttemperaturen eine schlechtere Verdampfung eintritt. Weiterhin wird eine Anreicherung bei Vollast durchgeführt, bei der die Drosselklappe voll geöffnet ist und eine erwünschte Erhöhung des Drehmoments erfolgt. Stöchiometrische Gemische hingegen werden meist bei betriebswarmen Motoren unter Teillast oder Leerlauf generiert.

Eine Weiterentwicklung der äußeren Gemischbildung ist die sogenannte Saugrohreinspritzung. Hierbei wird der Kraftstoff direkt im Saugrohr der Luft zugeführt. Dies geschieht zwischen Drosselklappe und Einlassventil. Neben der Reduzierung des Verbrauchs und der Schadstoffemission zeichnet diese Technologie eine genauere Kraftstoffmengenkontrolle zu den Betriebs- und Lastpunkten des Motors, sowie ein schnelleres Ansprechverhalten aus.

Nachteile der äußeren Gemischbildung entstehen zum einen bei Lastwechsel. Da der Zustand der Teillast durch Drosselung der Luftzufuhr eingeleitet wird, liegt ein verminderter Druck im Saugrohr bzw. im Zylinder vor. Der ansaugende Kolben, dessen Bewegungsenergie von der vorangegangen Verbrennung gewonnen wurde, muss aufgrund des Unterdrucks zusätzliche Arbeit verrichten. Dies bewirkt eine 
Verschlechterung des Wirkungsgrades des Ottomotors bei Lastwechsel [7]. Um einen dissipativ wirkenden Unterdruck zu verhindern, gibt es die Möglichkeit, unabhängig vom Lastzustand die gleiche Menge Luft anzusaugen. Das bedeutet aber, dass eine stöchiometrische Zusammensetzung des Kraftstoff-Luft-Gemisches bei Teillast nicht mehr möglich ist und eine Verbrennung bei hohem Luftüberschuss stattfindet. Da eine Zündung nur bis zu einem Luftverhältnis von $\lambda \approx 1,3$ sicher erfolgt [8], ist eine Ladungsschichtung erforderlich. Eine Ladungsschichtung hat im Gegensatz zum homogenen Gemisch keinen konstanten $\lambda$-Wert, sondern einen ortsabhängigen Wert. Hierbei läuft die Verbrennung in einer relativ fetten Zone nahe der Zündkerze ab, während in anderen Bereichen das Luftverhältnis entsprechend höher ist [8]. Eine solche inhomogene Kraftstoffverteilung im Brennraum zu erzielen, ist mit den genannten Verfahren nur bedingt möglich.

Eine bessere Lösung bietet eine Benzindirekteinspritzung mit geschichteter Zylinderladung [9]. Die Vorteile sind neben dem reduzierten Kraftstoffverbrauch und der Schadstoffemission ein besseres Ansprechverhalten bei Lastwechsel, sowie in der Kaltstart- und Warmlaufphase. Daher hat sich diese Technologie im PKW-Bereich durchgesetzt und ist fast ausschließlich in allen heutigen Automobilen zu finden. Bei diesem Verfahren handelt es sich um eine innere Gemischbildung, bei der der Kraftstoff unter konstanter Luftzufuhr direkt in den Brennraum eingespritzt wird. Bei der Benzindirekteinspritzung bestimmt der Zeitpunkt des Einspritzvorgangs den Zeitraum der Gemischbildung, wobei drei Betriebsarten unterschieden werden. Homogen stöchiometrische, homogen magere und geschichtete Betriebsart. Bei homogen stöchiometrischer Betriebsweise wird der Kraftstoff während des Ansaugtaktes eingespritzt und hat so in der Regel genügend Zeit, vollständig zu verdampfen. Die Gemischzusammensetzung erfolgt unter Lambdavorgabe und wird mit Hilfe der Drosselklappe geregelt. Auch im homogen mageren Betrieb wird der Kraftstoff innerhalb des Ansaugtaktes zugeführt und wird ebenfalls lambdagesteuert. Hierbei ist ein Wert von $\lambda>1,3$ sinnvoll, da sonst bei der Verbrennung eine hohe Konzentration von Stickoxiden, sogenannte $\mathrm{NO}_{\mathrm{x}}$, entsteht. Denh eigentlichen Vorteil der direkten Einspritzung bildet aber der Schichtbetrieb. Der Kraftstoff wird während des Kompressionshubs zeitlich kurz vor der Zündung eingespritzt. Ziel ist es, im Bereich der Zündkerzenelektrode ein zündfähiges Gemisch zu erzeugen, während der restliche Brennraum mit Luft und Abgas aus innerer und externer Abgasrückführung gefüllt ist (mageres Gemisch). In dieser Betriebsart wird die Drosselklappe nicht definiert geöffnet, was einen beinahe drosselfreien Ansaugvorgang zur Folge hat. Somit werden Ladungswechselverluste minimiert. Weiterhin sinken die Wandwärmeverluste aufgrund der lokal begrenzten Verbrennung.

Die Gemischvorbereitung bei den direkteinspritzenden Ottomotoren wird durch drei Verfahren generiert. Beim strahlgeführten Verfahren bewirkt ausschließlich der Kraftstoffstrahl die Gemischbildung ohne gezielte Unterstützung der Ladungsbewegung. Hierbei liegen die Einspritzdüse und die Zündelektrode dicht beieinander, was auch bei kleinen Einspritzmengen ein zündfähiges Gemisch garantiert. Dieses Verfahren 
kann homogene Gemische erzeugen, indem während der Kolbenabwärtsbewegung eingespritzt wird. Im Gegensatz dazu entsteht eine Ladungsschichtung bei einer späten Einspritzung kurz vor Erreichen des sogenannten oberen Totpunktes und liefert eine zündfähige Ladungsschicht am Zündort. Der obere Totpunkt (OT) bezeichnet den Punkt der höchsten Kompression und ist in der Motorentechnik der Bezugspunkt für die Kolbenstellung. Diese wird über die Winkelstellung des Kurbelzapfens der Kurbelwelle zum OT in Grad Kurbelwinkel angegeben ( $\left.{ }^{\circ} \mathrm{KW}\right)$. Man spricht z.B. von $20^{\circ} \mathrm{KW}$ vor OT, also die Stellung des Kolbens bei einem Winkel von $20^{\circ}$ bevor der obere Totpunkt erreicht ist. Die zweite Betriebsart ist das wandgeführte Verfahren, bei der eine Gemischbildung mit Hilfe der Brennraumwand erfolgt. In den meisten Fällen übt eine speziell geformte Kolbenmulde diese Funktion aus. Einspritzwinkel und Kolbenbewegung unterstützen hierbei eine zündfähige Gemischkonzentration in der Nähe der Zündkerze. Beim luftgeführten Verfahren handelt es sich um die dritte Möglichkeit der Direkteinspritzung. Der Kraftstoff soll hier durch eine kontrollierte, einlassgenerierte Ladungsbewegung zur Zündelektrode geführt werden. Dabei soll es keine Wandberührung mit dem Kraftstoff geben, sondern eine direkte Vermischung mit der Luft. Die Brennraumwand hat nur eine stabilisierende Funktion der im Ansaugvorgang erzeugten Strömung. Einspritzdüse und Zündkerze haben hier einen größeren Abstand. 

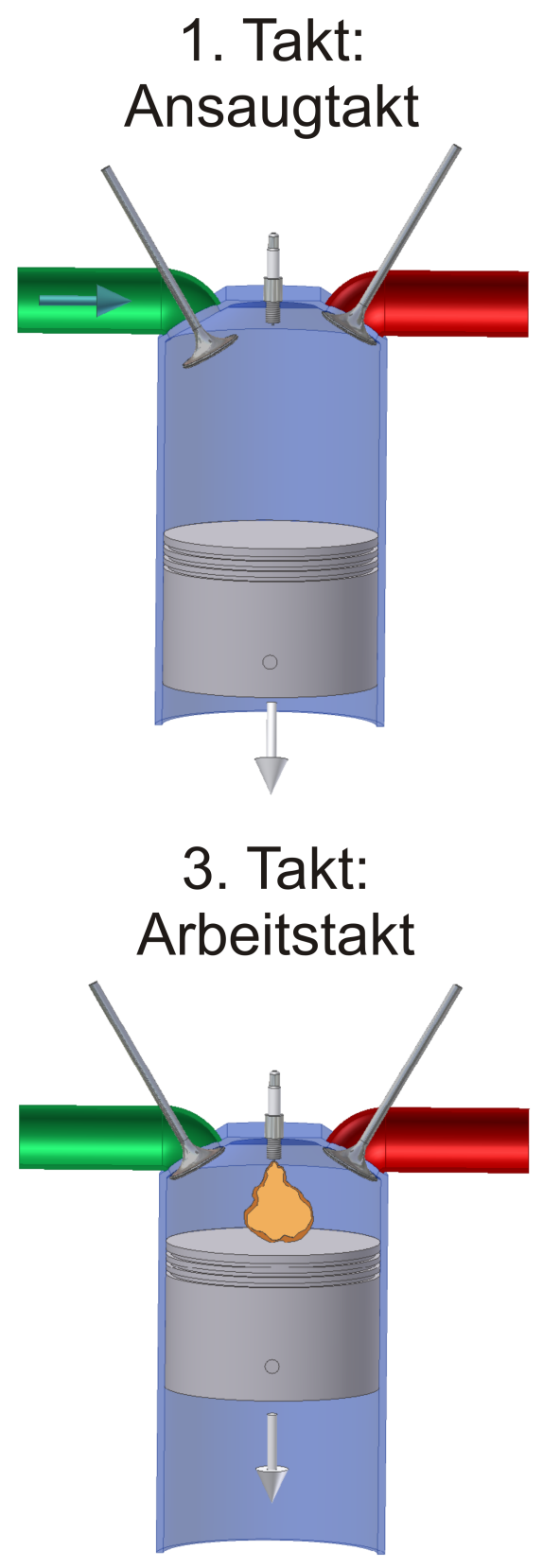

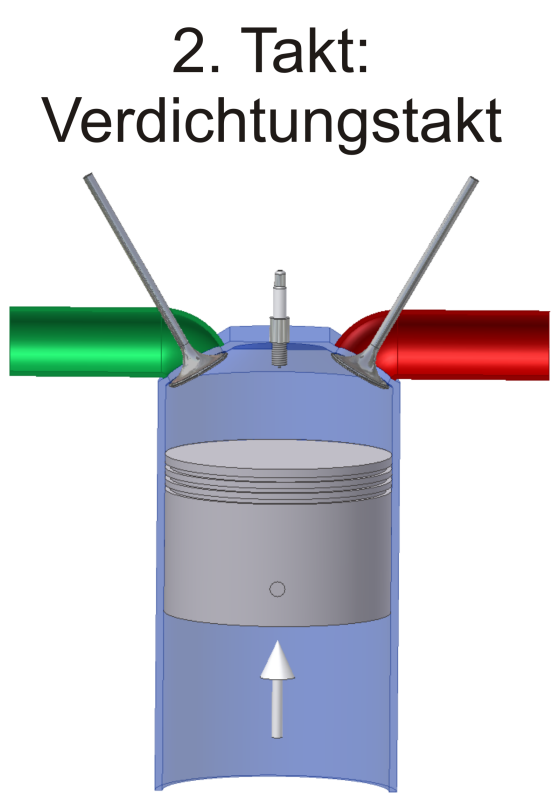

4. Takt: Ausstoßtakt

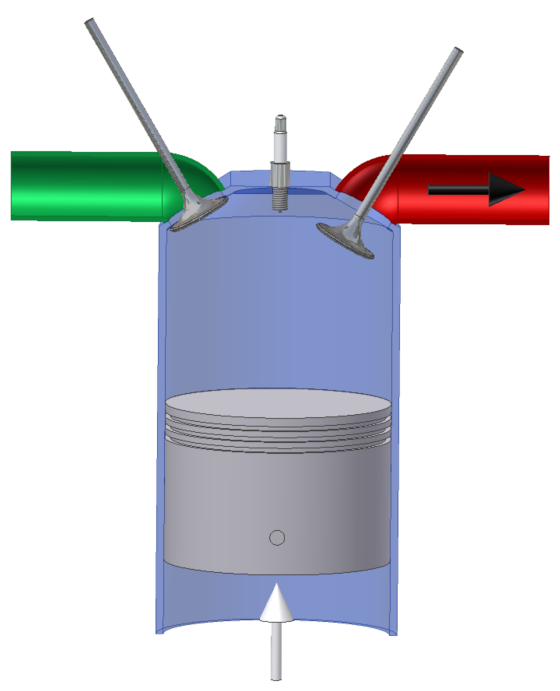

Abbildung 3.1: Grundprinzip des Viertakt-Ottomotors: Der Arbeitszyklus beginnt mit der Abwärtsbewegung des Kolbens. Der dabei entstehende Unterdruck saugt bei geöffnetem Einlassventil (links) das Gemisch über das Saugrohr (grün) in den Brennraum. Bei geschlossenen Ventilen wird das Gemisch durch die Aufwärtsbewegung des Kolben verdichtet und im Arbeitstakt zur Zündung gebracht. Aufgrund der frei werdenden Energie wird auf den Kolben Arbeit übertragen. Bei der nächsten Aufwärtsbewegung wird das Auslassventil geöffnet und das Abgas über das Auslassrohr (rot) ausgestoßen. 


\subsubsection{Zündung und Verbrennung}

Die motorische Verbrennung beschreibt eine schnelle Oxidation der reagierenden Stoffe unter Flammenerscheinung [9]. Eine stöchiometrische Verbrennung von IsoOktan verläuft nach folgender Reaktion:

$$
\mathrm{C}_{8} \mathrm{H}_{18}+12,5 \mathrm{O}_{2} \longrightarrow 8 \mathrm{CO}_{2}+9 \mathrm{H}_{2} \mathrm{O}
$$

Dabei wird ersichtlich, dass als Produkte einer Verbrennung Wasser und Kohlendioxid entsteht. Bei Verwendung von Kraftstoff und Luft werden allerdings weitere Produkte generiert. Dabei handelt es sich um verschiedene Kohlenwasserstoff- $(\mathrm{CH})$, Stickoxid- $\left(\mathrm{NO}_{\mathrm{x}}\right)$ und Schwefelverbindungen $\left(\mathrm{SO}_{\mathrm{x}}\right)$. Voraussetzung für eine rasche Verbrennung sind eine feine Kraftstoffzerstäubung und eine gleichmäßige Vermischung mit der Luft, um eine große Berührungsfläche zwischen den reagierenden Stoffen zu generieren. In Ottomotoren erfolgt die Zündung durch Fremdzündung mittels einer Zündkerze. Ein Zündfunken liefert die für die Verbrennung benötigte Aktivierungsenergie. Am Zündort entstehen durch Spaltung der Kohlenwasserstoffverbindungen freie Radikale, welche wiederum aktive Zentren für weitere anschließende Reaktionen bilden. Somit entsteht im Brennraum eine exotherme Kettenreaktion, die sich vom Zündort ausbreitet und Wärmeenergie freisetzt, die in mechanische Energie umgewandelt wird. Auf den Verlauf der Verbrennung haben allerdings verschiedene Faktoren einen entscheidenden Einfluss. Die Abhängigkeit der Zündtemperatur vom eingesetzten Kraftstoff-Luft-Gemisch entscheidet über eine schadstoffarme Verbrennung. Weicht der $\lambda$-Wert vom stöchiometrischen Verhältnis ab, so steigt die Zündtemperatur, da die überschüssigen Reaktionsstoffe nicht mehr reagieren. Eine Zündbereichserweiterung eines solchen Gemisches läßt sich nur durch eine Erhöhung der Zündtemperatur realisieren.

Eine Vergrößerung des Zündfunkens und dessen Lebensdauer ermöglicht eine Verbrennung von sehr mageren Gemischen. In diesem Fall werden aber höhere Zündenergien benötigt. Der Zündzeitpunkt ist ein weiterer Einflussfaktor für den Verbrennungsverlauf. Dieser ist abhängig von der Drehzahl, dem Druck, der Gemischtemperatur und dem sogenannten Verbrennungsluftverhältnis $\lambda_{V}$. Hier ist zu berücksichtigen, dass $\lambda_{V}$ ein momentanes Verbrennungsluftverhältnis ist, welches sich auf die innere Gemischbildung bezieht. Grundlage des Unterschieds zum zuvor definierten $\lambda$-Wert ist die Annahme, dass bei der äußeren Gemischbildung $\lambda$ aufgrund des homogen angesaugten Gemisches sowohl orts- als auch zeitunabhängig ist. Bei der inneren Gemischbildung hingegen variiert das Luftverhältnis je nach Lastbereich und Zustand der Motorbedingungen (kalter oder warmer Motor) und ist somit eine orts- und zeitabhänige Größe [7].

Eine weiterer wichtiger Faktor ist die Flammengeschwindigkeit, welche eng mit der Lage des Zündzeitpunktes verbunden ist. So ist z.B. ein guter Wirkungsgrad erreicht, wenn eine volle Entflammung des Gemisches kurz nach dem oberen Totpunkt vorliegt. Desweiteren spielt die Gemischzusammensetzung eine dominante Rolle für 
die Flammengeschwindigkeit. Um eine geringe Schadstoffemission zu erhalten, werden möglichst magere Gemische in den Brennraum eingespritzt. Bei Luftüberschuss $\left(\lambda_{V}>1\right)$ verringert sich die Flammengeschwindigkeit, was eine Vorverlegung des Zündzeitpunktes nach sich zieht. Ein fettes Gemisch hingegen erfordert einen späteren Zündzeitpunkt. Die Flammengeschwindigkeit ist bei $\lambda_{V}=0,9$ am größten [6]. Hier liegt auch die maximale Leistung des Motors. Der Kraftstoffverbrauch hingegen nimmt mit steigendem Luftverhältnis ab und hat sein Minimum im Bereich von $\lambda_{V}=1,2$. Darüber steigt der Verbrauch wieder wegen der geringeren Flammengeschwindigkeit, was zu einer verschleppten Verbrennung oder sogar Aussetzern führen kann. Im „fetten“ Bereich entsteht aufgrund des Luftmangels ebenfalls eine unvollständige Verbrennung. Auch die Schadstoffemission von Kohlenwasserstoffen hat das Minimum bei $\lambda \approx 1,1$ und steigt bei höherem Luftanteil wieder an. Dies ist darin begründet, dass die Brennraumwand eine Abkühlung des Gemisches hervorruft und somit die Flamme erlischt (unvollständige Verbrennung). Auch unterhalb von $\lambda_{V}=1,1$ steigt die CH-Emission wegen Luftmangels an. Ein anderes Verhalten zeigt Stickoxid. Die $\mathrm{NO}_{\mathrm{x}}$-Emission nimmt mit steigender Sauerstoffkonzentration $\left(\mathrm{O}_{2}\right)$ und maximaler Verbrennungstemperatur zu. Bei $\lambda=1,1$ hat die $\mathrm{NO}_{\mathrm{x}}$-Emission ein Maximum, da hier eine hohe $\mathrm{O}_{2}$-Konzentration, aber vor allem eine höhere Spitzentemperatur vorherrscht. Bei mageren Gemischen steigt zwar der $\mathrm{O}_{2}$-Anteil, aufgrund des Absinkens der Spitzentemperatur durch Verdünnung des Gemisches hingegen fällt die $\mathrm{NO}_{\mathrm{x}}$-Emission wieder ab.

Der Verlauf einer gewünschten normalen ottomotorischen Verbrennung beginnt mit der Homogenisierung und Verdichtung des Gemisches. Danach wird kurz vor Erreichen des OT die Verbrennung mit Hilfe eines Zündfunkens eingeleitet. Innerhalb des Brennverfahrens entstehen allerdings auch anormale Verbrennungen. Das heißt, es finden Verbrennungen statt, die nicht durch die im Brennraum ausbreitende Flammenfront initiiert werden. Zwei dieser Vorgänge sind das Klopfen und die Glühzündung. Eine klopfende Verbrennung entsteht durch zu starke Druckschwankungen im Brennraum, welche zu einer spontanen Selbstzündung des Restgases (Gas, welches noch nicht von der Flammenfront erfasst wurde) führen. Die ausbreitende Flamme übt auf das umliegende Restgas eine Kompression aus und erhöht somit dessen Temperatur. Diese übersteigt dabei die Zündgrenztemperatur und löst die Selbstzündung aus. Die Selbstzündung generiert eine annähernd isochore Restgasverbrennung und erzeugt dabei hohe Druckgradienten, welche sich in Form von Druckwellen ausbreiten und als klopfendes Geräusch wahrgenommen werden kann. Starken Einfluss auf das Klopfen hat die Zusammensetzung des Kraftstoffs. Die Neigung zur Selbstzündung eines Kraftstoffes wird über die sogenannte Oktan-Zahl (OZ) beschrieben. Diese Maßzahl liegt im Bereich von 0 (klopffreudiger Kraftstoff, wie z.B. n-Heptan) bis 100 (klopffester Kraftstoff, Iso-Oktan). So hat z.B. Superbenzin eine Oktan-Zahl von 95 und Normalbenzin $91^{1}$. Dieses charakteristische Merkmal eines Kraftstoffes

\footnotetext{
${ }^{1}$ laut DIN EN 228
} 
wird in der Literatur als Klopffestigkeit bezeichnet.

Bei der Glühzündung lösen extrem heiße Zonen, sogenannte „hot spots“, eine Fremdverbrennung aus. Diese heißen Zonen werden meist durch Verbrennungsrückstände (Rußablagerungen) gebildet, welche sich an den Auslassventilen und den Kolbenböden abgelagert haben. Deren Temperatur ist deutlich höher als die Selbstentzündungstemperatur und damit Auslöser der Selbstzündung. Auch hier treten hohe Druckwellen auf. Beide Brennverläufe sind unerwünscht, da sie eine hohe thermische und mechanische Belastung für die brennraumbildenden Bauteile sind.

\subsubsection{Homogene Kompressionszündung}

Um eine weitere Verringerung sowohl des Kraftstoffverbrauchs als auch der Schadstoffemission zu erzielen, werden die oben erwähnten Technologien stetig weiterentwickelt. Eine vielversprechende Alternative stellt die homogene Selbstzündung dar, welche 1949 erstmals Hermann Teegen [10, 11] in Form des Lohmann-Hilfsmotors realisierte. Später wurde dieses Konzept von Onishi et al. [12] wieder aufgegriffen und als active thermal atmosphere combustion (ATAC) bezeichnet.

In der Entwicklung des Ottomotors ist eine Selbstzündung durch das oben erwähnte Klopfen bekannt. Basierend auf den damit einhergehenden mechanischen Belastungen, war es für die Entwickler das vorrangige Ziel, eine Selbstzündung zu vermeiden. Die in diesem Verfahren gewollte Selbstzündung unterscheidet sich jedoch einerseits durch eine erhebliche Verdünnung des Gemisches, was eine Verringerung der lokalen Wärmefreisetzung hervorruft. Des Weiteren findet, im Gegensatz zum Klopfen, eine gleichmäßige, im gesamten Zylinder auftretende Selbstzündung statt. Die beim Klopfen entstehenden Druckwellen bleiben somit aus. Das als HCCI (engl. „Homogeneous Charge Compression Ignition") bekannte Verfahren arbeitet mit einem homogenen Kraftstoff-Luft-Gemisch. Die durch das Verdichten ansteigende Temperatur löst dabei eine Selbstzündung aus. Die Zündwilligkeit des Gemisches wird mit Hilfe einer Abgasrückführung unterstützt. Durch einen zugeführten Anteil an heißem Abgas wird die Gemischtemperatur schon vor der Kompression erhöht. Desweiteren liegen im Abgas von der Vorverbrennung übrig gebliebene hochreaktive Zwischenprodukte, sogenannte Radikale, vor. In Wechselwirkung mit dem Frischgas beschleunigen diese Radikale die Zündung des klopffesten Kraftstoffes und lösen so die Radikalkettenreaktion aus. Aufgrund der Abgasrückführung entsteht ein sehr mageres Gemisch, wodurch ein niedriger Kraftstoffverbrauch realisiert wird. Durch das magere und mit Abgas verdünnte Gemisch ist die Verbrennungstemperatur niedrig, was zu der geringen $\mathrm{NO}_{\mathrm{x}}$-Bildung führt. Auch die Rußemission wird mit der guten Vormischung deutlich reduziert. Dieser Sachverhalt hat den Effekt, dass eine aufwendige und kostspielige Abgasnachbehandlung nicht mehr benötigt wird. Ein weiterer Vorteil des HCCI-Motors ist die hohe Effizienz, welche durch die hohe Kompressionsrate begründet ist, die für die Selbstzündung benötigt wird.

Wichtig ist hierbei, dass die Verbrennung kontrolliert abläuft. Bei Teillast, bei der ei- 
ne stöchiometrische Verbrennung stattfindet, funktioniert das HCCI-Konzept recht gut. Schwierigkeiten stellen sich aber bei Variation der Motorgeschwindigkeit bzw. Lastwechsel, in der Kaltstartphase oder unter Vollast ein. Bei einem zu fetten Gemisch erfolgt eine zu rasche Verbrennung und löst das unerwünschte Klopfen aus. Liegt ein zu mageres Gemisch vor, so entsteht eine unvollständige Verbrennung und es kommt zu Fehlzündungen. Generell ist die extrem schnelle Verbrennung im HCCI-Motor ein großes Problem für die dynamische Steuerung der Selbstzündung. Herrscht im Zylinder eine hohe Temperatur, so kann es passieren, dass das Gemisch an vielen Orten gleichzeitig zündet. Idealerweise sollte das Gemisch im Motor zwischen $15^{\circ} \mathrm{KW}$ und $10^{\circ} \mathrm{KW}$ vor OT beginnen zu zünden und die Verbrennung bis nach dem OT andauern. Bei einer vorzeitigen Verbrennung hat das heiße Gas zu langen Kontakt mit den Zylinderwänden und es treten zu hohe Wärmeverluste auf. Eine spätere Verbrennung hat Leistungsverluste zur Folge. Aus diesen Gründen ist es schwierig, einen alltagstauglichen Motor mit reinem HCCI-Brennverfahren zu generieren. Daher sind heutige HCCI-Motoren eine Kombination der verschiedenen Verbrennungsverfahren [13]. Um beim Ottomotor eine Selbstzündung zu gewährleisten, ist eine gezielte Menge an zurückgeführtem Abgas notwendig. In Kombination mit einer Direkteinspritzung läßt sich am besten ein homogenes Gemisch in der Kompressionsphase zum richtigen Zeitpunkt zur Selbstzündung bringen. Eine frühe Einspritzung in der Ansaugphase bildet beim Ottomotor den idealen Zeitbereich. Aufgrund des auf den Teillastbereich (niedriger Last- und Drehzahlbereich) beschränkten HCCI-Betriebes, wird der Ottomotor außerhalb dieses Bereiches konventionell mit Fremdzündung betrieben. 

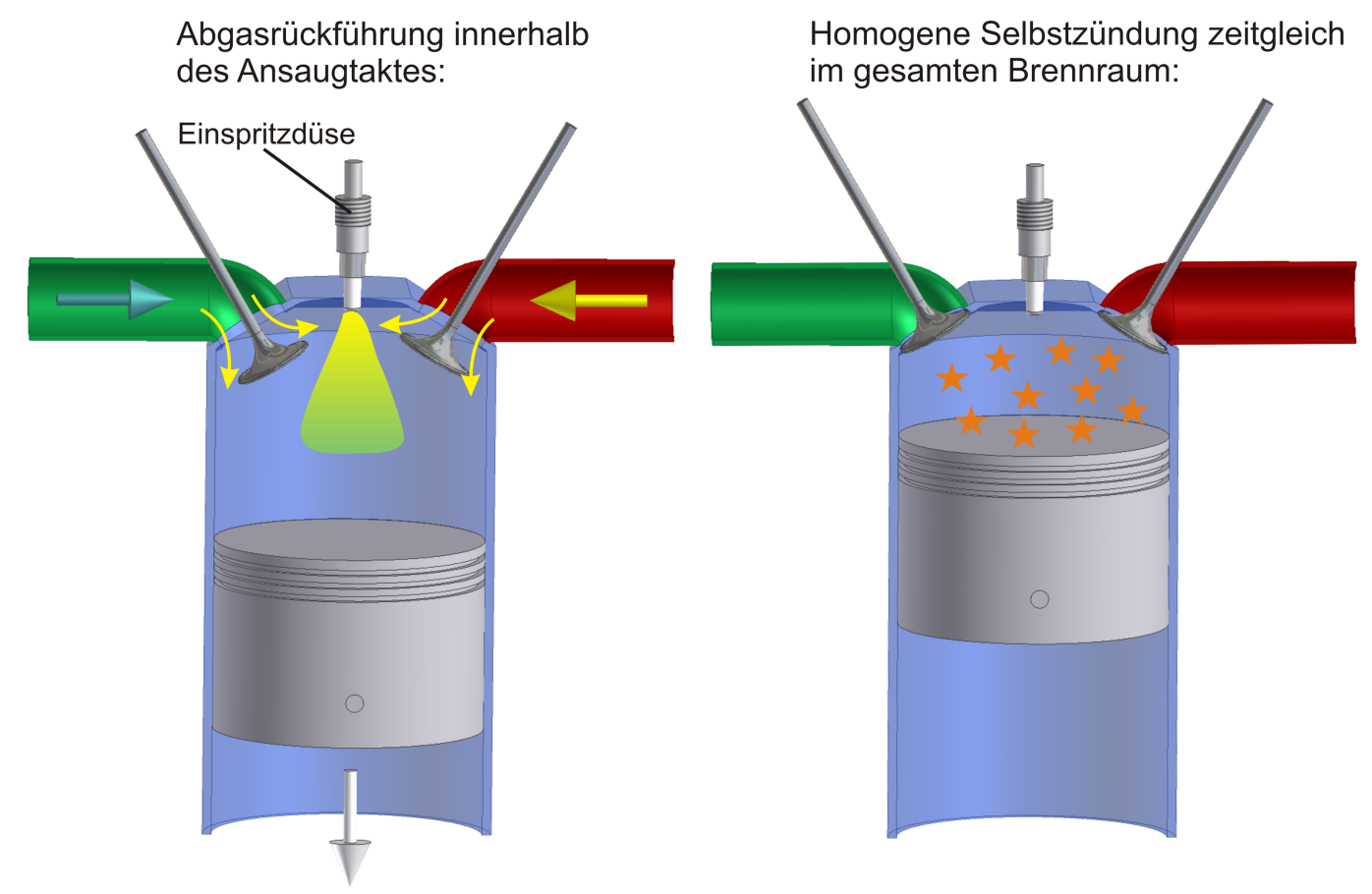

Abbildung 3.2: Der im Ansaugtakt zurückgeführte Abgasanteil (gelbe Pfeile Auslaßseite) erhöht die Gemischtemperatur und beschleunigt durch Radikalreste die Selbstzündung. Diese findet im Brennraum gleichmäßig statt. 


\section{Kapitel 4}

\section{Wissenschaftliche Grundlagen des angewandten Messverfahrens}

In diesem Kapitel werden sowohl physikalische Phänomene als auch molekülspezifische Eigenschaften vorgestellt, mit deren Hilfe ein Messverfahren zur Temperaturbestimmung in der Motordiagnostik entwickelt wurde. Die Erfassung physikalischer Größen von Verbrennungsvorgängen ist eine Herausforderung, da diese zum einen sehr dynamisch sind, und zum anderen sich der Zugang zu diesen Informationen aufgrund der motorischen Umgebung als recht schwierig erweist. Nur ein spektroskopisches Verfahren kann hier herangezogen werden, um minimal invasiv zu wirken und Messungen unter realen Bedingungen zu ermöglichen. Parallel zu den physikalischen Vorgängen vollziehen sich chemische Reaktionen innerhalb der Verbrennung, welche in die Betrachtungen miteinbezogen werden müssen. Weiterhin gilt es, dass angewandte Verfahren mit den Eigenschaften des Moleküls, welches die spektroskopischen Informationen liefert, abzustimmen. Die Vereinigung dieser einzelnen Bereiche bildet die wissenschaftliche Basis für das in dieser Arbeit vorgestellte Meßverfahren.

\subsection{Spektroskopische Verfahren in der Motordiagnostik}

Dieses Kapitel soll einen Überblick über die spektroskopischen Verfahren geben, die zur Temperaturbestimmung herangezogen wurden. Schulz et al. [14] entwickelte ein Verfahren, welches auf der Rayleigh-Streuung basiert. Ein Nachteil ist hierbei, dass eine geringe Intensität des gestreuten Laserlichts vorliegt. Durch Streuung an den Brennraumwänden und Kraftstofftröpfchen bzw. Staubpartikeln entsteht ein starkes Hintergrundsignal, welches ein sehr geringes Signal-zu-Rausch-Verhältnis produziert und somit eine Anwendung erschwert. Zuvor wurde von Zur Loye et al. [15] die Raman Spektroskopie an Verbrennungsmotoren angewandt. Auch hier stellt die geringe Intensität ein großes Problem dar und des Weiteren erfordern die geringen Abstände der Stokes- und Antistokes-Linien eine hohe spektrale Auflösung. Palmer et al. [16] nutzte Laserinduzierte Fluoreszenz (LIF) von NO-Radikalen, um 
im Stoßrohr die Temperatur zu bestimmen. Arnold et al. [17] untersuchte ebenfalls mit LIF OH-Radikale in Flammen. Diese Radikale entstehen jedoch erst während des Verbrennungsvorgangs, so dass mit diesen Verfahren die Gemischtemperatur vor der Zündung nicht erfasst werden kann. Die Temperaturbestimmung auf Basis der gleichzeitigen Abfrage der Besetzung zweier Energieniveaus des elektronischen Grundzustands macht den gleichzeitigen Einsatz von zwei Lasern notwendig und ist experimentell sehr aufwendig. Einecke et al. [18] nutzte dieses Verfahren unter Verwendung von 3-Pentanon und erzielte eine gute Verifizierung der Temperatur. Für die Sensorentwicklung ist dies zum einen konstruktiv recht komplex und aufgrund des zweiten Lasers entstehen auch höhere Kosten. Neben der herkömmlichen Abfrage der thermisch angeregten Zustandsbesetzungen im elektronischen Grundzustand gibt es auch die Möglichkeit, eine Zwei-Linien-Fluoreszenzmethode anzuwenden, die auf der Messung der Vibrationszustandsbesetzungen eines Tracermoleküls basiert. Die in dieser Arbeit vorgenommenen Voruntersuchungen an einer Zelle unter motorrelavanten Bedingungen [19] gaben eine gute Übereinstimmung zwischen dieser zuletzt genannten Methode und den in dieser Arbeit gestellten Ziele.

\subsection{Laserinduzierte Fluoreszenz}

Als spektroskopische Methode für das vorgestellte Messverfahren erweist sich die Laserinduzierte Fluoreszenz als geeignet. Dieses Phänomen gewährleistet ausreichende Signalintensitäten, um auch geringe Konzentrationen zu detektieren. Dies ist ein großer Vorteil gegenüber zum Beispiel der Ramanspektroskopie, welche sehr viel geringere Intensitäten generiert. Besonders in der recht komplexen Umgebung eines Brennraums ist dieses sensitive Verfahren ein vielversprechendes Hilfsmittel, um Einblicke in die technische Verbrennung zu bekommen.

Durch Bestrahlung einer Substanz mit einem Laser kann es geschehen, dass das Photon des Laserstrahls von dieser absorbiert wird und ein Übergang in ein elektronisch höheres Niveau stattfindet. Bedingung hierfür ist, dass die Energie des absorbierten Photons die Energiedifferenz des Grundzustands und des angeregten Zustands ist. Der nachfolgende Übergang zurück in den Grundzustand unter spontaner Emission eines Photons wird als Fluoreszenz bezeichnet. Die Fluoreszenz ist eine Unterkategorie der Lumineszenz, welche noch die Phosphoreszenz beinhaltet. Bei den Übergängen handelt es sich in den meisten Fällen um einen Singulett-Singulett-Übergang vom Grundzustand $S_{0}$ in den nächsthöheren angeregten Zustand $S_{1}$. Das Gesamtspinmoment ist in diesem Fall $S=0$, was gleichbedeutend ist mit der Antiparallelität der Spins der bindenden Elektronen (das angeregte Elektron hat entgegengesetzten Spin zum Bindungspartner im Grundzustand). Dieser Relaxationsprozess konkurriert mit anderen Prozessen. Beim Übergang in ein höheres Niveau ist die Ähnlichkeit des Schwingungniveaus im Grundzustand $\nu^{\prime \prime}$ mit dem Schwingungsniveau $\nu^{\prime}$ im angeregten Zustand entscheidend dafür, in welches Schwingungsniveau innerhalb von 
$S_{1}$ das Elektron übergeht. Dies wird durch das Franck-Condon-Prinzip beschrieben und quantenmechanisch durch das Überlappungsintegral

$$
q_{\nu^{\prime} \nu^{\prime \prime}}=\int \Psi_{\nu^{\prime}} \cdot \Psi_{\nu^{\prime \prime}} d r
$$

mit den Wellenfunktionen $\Psi_{\nu^{\prime}}$ und $\Psi_{\nu^{\prime \prime}}$ ausgedrückt. Abbildung 4.1 veranschaulicht diese Übergangsbedingung.

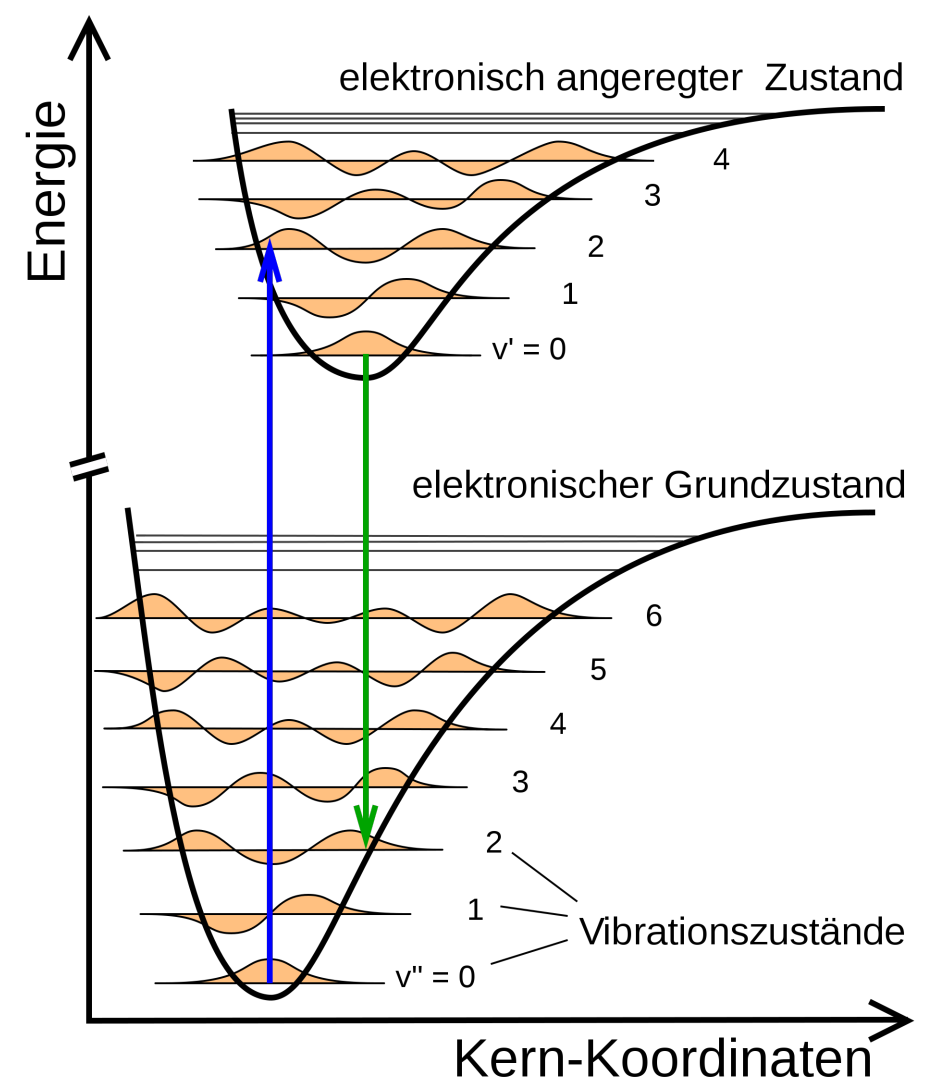

Abbildung 4.1: Die blaue Linie stellt den Übergang vom Grundzustand in einen angeregten Zustand dar. In welchem Vibrationsniveau die Anregung stattfindet, hängt davon ab, welche Wellenfunktionen (bei nahezu konstantem Kernabstand) die größte Überlappung bilden. Dieselbe Bedingung herrscht beim Übergang zurück in den elektronischen Grundzustand unter Aussendung eines Photons (grüne Linie).

Wenn nun in ein höheres Schwingungsniveau angeregt wird, so erfährt das Elektron eine strahlungslose Relaxation in den Schwingungsgrundzustand in $S_{1}$ durch Stöße mit benachbarten Molekülen. Wenn es diesen Zustand erreicht hat, findet 
der Übergang in den Grundzustand unter Aussendung eines Photons statt. Weitere Relaxationsprozesse sind das sogenannte Internal Crossing (IC oder innere Umwandlung), oder Intersystem Crossing (ISC oder Interkombination). Beim Internal Crossing entsteht ein Übergang direkt aus einem höheren Schwingungsniveau im elektronisch angeregten Zustand in einen niederenergetischen Zustand oder in den Grundzustand ohne Umkehr des Elektronenspins. Das Intersystem Crossing ist ein Singulett-Triplett-Übergang $\left(S_{1} \rightarrow T_{1}\right)$ ausgehend von einem angeregten SingulettZustand. Hierbei ist das Gesamtspinmoment $T_{1}=1$, also zwei ungepaarte Spins. Dieser Übergang ist strahlungslos und es wird somit keine Energie an die Umgebung abgegeben. Stattdessen wechselt das Elektron unter Spinumkehr in einen metastabilen Zustand, den Triplettzustand. Innerhalb dieses Zustands entsteht wiederum eine Relaxation in den Schwingungsgrundzustand. Ein Übergang in den SingulettZustand ist allerdings nur unter einer erneuten Spinumkehr möglich. Genau wie die erste Spinumkehr ist diese quantenmechanisch verboten. Dass dieses Phänomen trotzdem mit einer gewissen Wahrscheinlickeit auftritt, basiert auf Spin-BahnWechselwirkungen. Der Übergang $T_{1} \rightarrow S_{0}$ erfolgt unter Emission eines Photons und stellt die zuvor erwähnte Phosphoreszenz dar. Aufgrund der verbotenen Spinumkehr hat dieser Zustand eine längere Verweildauer, wodurch die Energie eines Ensembles nur allmählich abgegeben wird. Daher resultiert das bekannte lange Nachleuchten bei der Phosphoreszenz.

Ein weiteres für die Fluoreszenzuntersuchungen wichtiges physikalisches Phänomen ist die Fluoreszenzlöschung durch artverwandte oder reaktive Moleküle. Dieser auch als Quenching bekannte Vorgang beruht auf einer strahlungslosen Energieabgabe an einen molekularen Stoßpartner. Besonders Sauerstoff ist ein hochreaktiver Stoßpartner und kann zu einer erheblichen Verkürzung der Lebensdauer des angeregten Zustands beitragen. Dies offenbart sich in der sogenannten Fluoreszenzlebensdauer $\tau_{f}$. Dies ist die Zeit, in der sich ein Molekül im oberen Niveau befindet. Die Lebendauer dieses Zustandes liegt im Bereich von $10^{-10}$ bis $10^{-5}$ s. Diese quantenmechanischen Phänomene können mit Hilfe eines sogenannten Jablonski-Diagramms (siehe Abbildung 4.2) dargestellt werden. Aufgrund der Relaxationsprozesse entsteht eine Rotverschiebung des Fluoreszenzspektrums gegenüber dem Absoptionsspektrum. Dieses Phänomen wird auch als Stokes-Shift bezeichnet. 


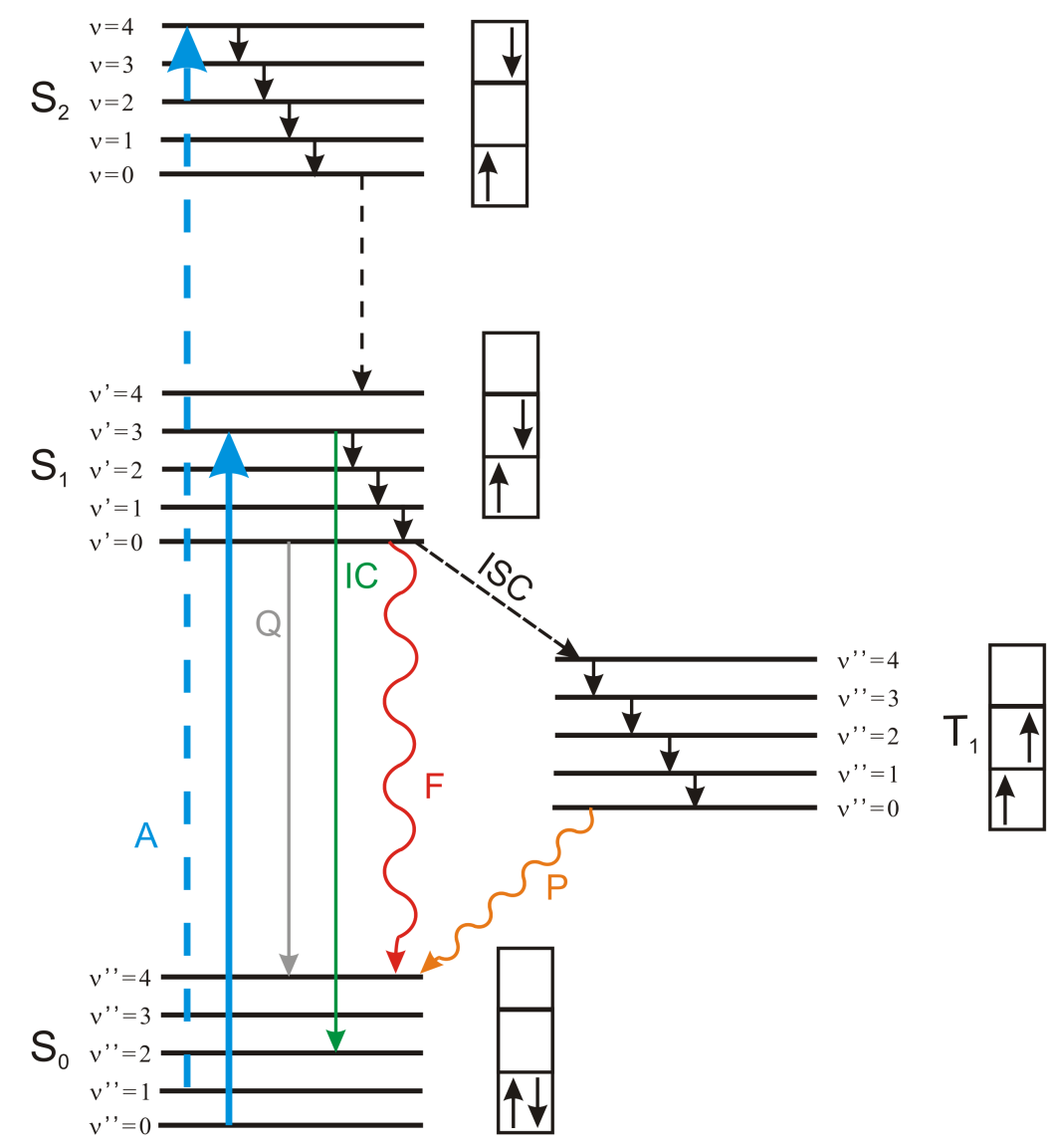

Abbildung 4.2: Mit Hilfe des Jablonski-Diagramms können die unterschiedlichen quantenmechanischen Ereignisse zusammengefasst werden. Die Absoption $A$ kann auch in höhere elektronische Niveaus stattfinden. Neben der Fluoreszenz $F$ und der Phosphoreszenz $P$ existieren auch strahlungslose Prozesse. Dazu gehören das Quenchen $Q$ oder das Internal Crossing $I C$. Ein weiterer strahlungsloser Übergang ist das Intersystem Crossing ISC, bei dem sich die Spinrichtung ändert. Der Spin des angeregten Elektrons ist am jeweils rechten Pfeil zu erkennen. 
Diese Wechselwirkung von elektromagnetischer Strahlung mit einem Atom oder Molekül kann näherungsweise über ein Zwei-Niveau-Modell beschrieben werden. Für ein von einem Laser bestrahlten Molekül hängt die Wahrscheinlichkeit der Absorption $W_{12}$ des Laserphotons und die stimulierte Emission $W_{21}$ von verschiedenen Faktoren ab. Neben dem zeitlichen Verlauf des Laserpulses $I(t)$ und dem in Gleichung 4.1 beschriebenen Überlappungsintegral $q_{\nu^{\prime} \nu^{\prime \prime}}$ hat das spektrale Profil des Laserstrahls und die spektrale Breite des Absorptionsspektrums einen entscheidenden Einfluss. Diese Wahrscheinlichkeiten werden mit den sogenannten Einsteinkoeffizienten für die Absoption $B_{12}$ und die stimulierte Emission $B_{21}$ durch

$$
\begin{aligned}
& W_{12}=B_{12} \cdot I(t) \cdot q_{\nu^{\prime} \nu^{\prime \prime}} \\
& W_{21}=B_{21} \cdot I(t) \cdot q_{\nu^{\prime} \nu^{\prime \prime}}
\end{aligned}
$$

beschrieben. Die spontane Emission ist unabhängig vom äußeren Strahlungsfeld und durch den Einsteinkoeffizienten $A_{21}$ gegeben. Für die Gesamtbesetzungsdichte $N$ gilt

$$
N=N_{1}(t)+N_{2}(t)
$$

mit den Besetzungszahlen $N_{1}$ für den Grundzustand und $N_{2}$ für den angeregten Zustand. Hierbei soll die Bedingung

$$
N_{2}(t=0)=0
$$

gelten. Unter der Einbeziehung der Quenchingrate $Q_{21}$ ergibt sich für den angeregten Zustand

$$
\frac{d N_{2}(t)}{d t}=W_{12} N_{1}(t)-N_{2}(t)\left[W_{21}(t)+A_{21}+Q_{21}\right]
$$

Bei einer Laseranregung mit angenommenen Rechteckpuls und einer zeitlichen Länge von $\tau$ besteht für die zeitliche Entwicklung des angeregten Zustands während der Laseranregung $(0<t<\tau)$ folgender Zusammenhang

$$
N_{2}(t)=\frac{N \cdot W_{12}}{A_{21}+Q_{21}}\left(1-e^{-\left(A_{21}+Q_{21}\right) t}\right)
$$

wobei $W_{12}$ und $W_{21} \ll\left(A_{21}+Q_{21}\right)$ und die Bedingung aus Gleichung 4.4 gilt. Nach Abklingen des Laserpulses ergibt sich für die Besetzung

$$
N_{2}(t)=N(\tau) \cdot e^{\left(A_{21}+Q_{21}\right)(t-\tau)}
$$

und für die gesamte Fluoreszenzintensität $F$ aus einem Volumen $V$ 


$$
\begin{aligned}
F= & \int_{0}^{\infty} N_{2}(t) \cdot A_{21} \cdot V d t \\
= & \int_{0}^{\tau} A_{21} \frac{N \cdot W_{12}}{A_{21}+Q_{21}}\left[1-e^{-\left(A_{21}+Q_{21}\right) t}\right] V d t \\
& +\int_{\tau}^{\infty} A_{21} \cdot N_{2}(\tau) \cdot e^{-\left(A_{21}+Q_{21}\right)(t-\tau)} V d t .
\end{aligned}
$$

Der Term $\Phi=A_{21} /\left(A_{21}+Q_{21}\right)$ beschreibt die Quantenausbeute der Fluoreszenz. Unter der Betrachtung, dass die Laserintensität einem Rechteckpuls mit $E=I \cdot \tau$ folgt, erhält man nach Integration für die gesamte Fluoreszenzintensität

$$
S_{f}=B_{12} \cdot E \cdot q_{\nu^{\prime} \nu^{\prime \prime}} \cdot N \frac{A_{21}}{A_{21}+Q_{21}}
$$

\subsection{Emissionsspektroskopie von Molekülen}

Moleküle bestehen aus einer Anzahl von $N$ Atomen und besitzen somit mehrere Freiheitsgrade. Für lineare Moleküle (Moleküle, deren Atome auf einer Molekülachse liegen) existieren 2 Rotations- und $3 N-5$ Schwingungsfreiheitsgrade. Nichtlineare Moleküle haben 3 Rotations- und $3 N-6$ Schwingungsfreiheitsgrade. Diese Freiheitsgrade unterscheiden sich in ihrer Energie und können in Spektren identifiziert werden. Da die Rotationsfreiheitsgrade energetisch sehr nah beieinander liegen, ist für ein Rotationsspektrum eine sehr hohe Auflösung notwendig. Auf diese wird aber nicht weiter eingegangen, da sie auf das vorgestellte Verfahren keinen entscheidenden Einfluss haben. Der Vibrationszustand der Moleküle besteht aus unterschiedlichen Arten von Schwingungen, zum Beispiel symmetrische und antisymmetrische Streckschwingungen (Valenzschwingungen) und Deformationsschwingungen, und wird durch die in Kapitel 4.2 eingeführten Schwingungsniveaus beschrieben. Bei einer Anregung aus einem elektronischen Grundzustand findet ein Übergang nach dem Franck-Condon-Prinzip statt. Bei Molekülen entsteht innerhalb dieses angeregten Zustands eine schnelle Umverteilung der Energie auf andere Schwingungsmoden. Dieser strahlungslose Prozess wird als intramolekulare Schwingungsumverteilung (engl. intramolecular vibrational redistribution, IVR) bezeichnet und die Besetzung der einzelnen Moden ist eine von der Molekülstruktur abhängige Größe. Nach der Umverteilung der Energie hat das Molekül einen thermodynamischen Gleichgewichtszustand erreicht. Die Zeit, die benötigt wird, um den Gleichgewichtszustand herzustellen, liegt im Pikosekunden- oder sogar im Femtosekunden-Bereich [20]. Aufgrund der längeren Fluoreszenzlebensdauer im Nanosekunden-Bereich, findet die Emission aus dem umverteilten, angeregten Zustand statt. Eine Bevölkerung der einzelnen Schwingungsniveaus im thermodynamischen Gleichgewicht ist von der 
Temperatur abhängig. Aus den Besetzungen der Schwingungsniveaus, die nach der statistischen Physik durch eine Boltzmann-Verteilung beschrieben werden kann, ist es somit möglich, eine Temperaturinformation zu extrahieren.

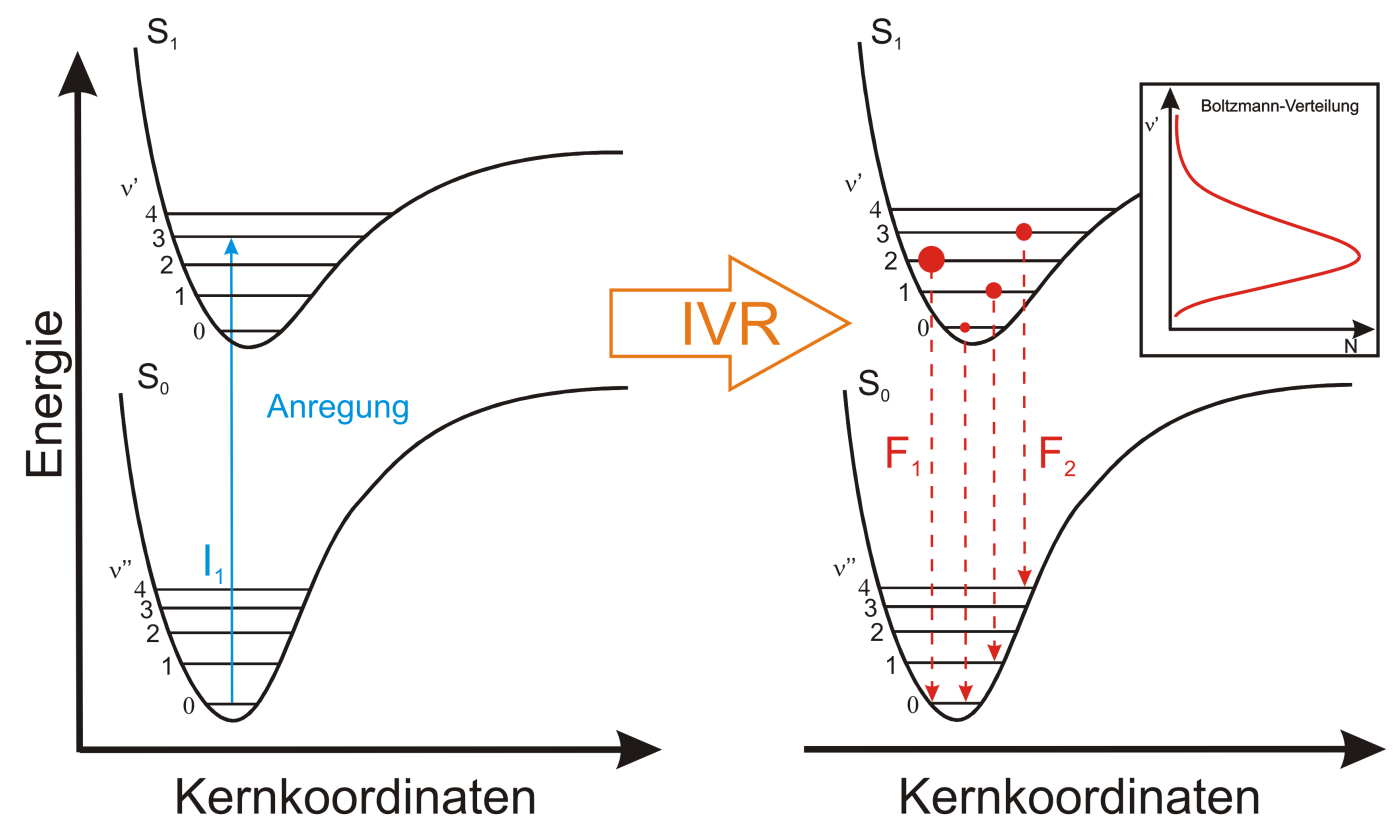

Abbildung 4.3: Nach der Anregung wird die Energie auf einzelne Schwingungsniveaus umverteilt (IVR). Diese ist deutlich kürzer als die Fluoreszenzlebensdauer. Die unterschiedlichen Besetzungen der Schwingungsniveaus ist in der Abbildung durch Kreise mit verschiedenen Radien schematisch dargestellt. Aus diesen Niveaus heraus kann ein elektronischer Übergang unter Emission von Fluoreszenz entstehen.

Die Moleküle besitzen durch die genannte Besetzung der Schwingungsniveaus somit ein Elektronen-Bandenspektrum, welches für die Temperaturbestimmung herangezogen werden kann. Hierbei wird ausgenutzt, dass die Intensität der einzelnen Banden die Besetzung der Schwingungsniveaus widerspiegelt. Die detektierte Fluoreszenzintensität $F$ eines bestimmten Schwingungszustandes ist durch die Gleichung

$$
F=c \cdot I \cdot V \cdot \sigma \cdot \Phi \cdot N_{0} \cdot e^{-\frac{E}{k_{B} T}}
$$

beschrieben. Bei den Faktoren handelt es sich um einen Faktor $c$, welcher die geometrischen und optischen Eigenschaften des Versuchsaufbaus beschreibt (auch als Setup-Faktor bezeichnet), die Laserintensität $I$, das Detektionsvolumen $V$, den Absorptionskoeffizienten $\sigma$, die Quantenausbeute $\Phi$, die Teilchenzahl im Grundzustand $N_{0}$, die Energie $E$, der Boltzmann-Faktor $k_{B}$ und die Temperatur $T$. 


\subsection{Reaktionskinetik der Verbrennung}

Die chemischen Reaktionsmechanismen der Verbrennung sind sehr komplex. Die Oxidation organischer Verbindungen vollzieht sich in der Regel in einer Aneinanderreihung von mehreren Elementarreaktionen. In der ersten Stufe der Kettenreaktion werden aus den stabilen Ausgangsprodukten aktive Zwischenprodukte, sogenannte Radikale, gebildet. Aufgrund der Stabilität wird eine hohe Aktivierungsenergie benötigt, um diesen Prozess zu initiieren. Daher reagiert ein Gemisch aus Kohlenstoffen und Sauerstoff nur sehr langsam bei Temperaturen unterhalb von $200^{\circ} \mathrm{C}$ [21]. Bei höheren Temperaturen erfolgt dann die Oxidation mit den Endprodukten CO, $\mathrm{H}_{2} \mathrm{O}$ und $\mathrm{CO}_{2}$. Da bei der Verbrennung allerdings keine stöchiometrische Reaktion vorherrscht, entstehen auch noch andere Produkte wie zum Beispiel Stickoxide $\left(\mathrm{NO}_{\mathrm{x}}\right)$ und Schwefeloxide $\left(\mathrm{SO}_{\mathrm{x}}\right)$.

Die Radikalbildung ist eine reversible Reaktion, das bedeutet, dass Radikale gebildet und abgebaut werden. Eine Kettenabbruchreaktion liegt vor, wenn sich ein Gleichgewicht zwischen Bildung und Abbau eines Produkts einstellt. Vor allem das gebildete OH-Radikal ist ein entscheidender Initiator für die Oxidation der Kohenstoffverbindungen. So wird zum Beispiel $\mathrm{CO}$ zu $\mathrm{CO}_{2}$ oxidiert

$$
\mathrm{CO}+\mathrm{OH} \leftrightarrow \mathrm{CO}_{2}+\mathrm{H}
$$

Ein stark verkürzter Oxidationsmechanismus des Modellkraftstoffs Methanol soll einen übersichtlichen Einblick in diese weitaus komplexere Kettenreaktion geben. Für eine Startreaktion mit dem OH-Radikal ergibt sich folgendes Reaktionsschema

$$
\mathrm{CH}_{3} \mathrm{OH}+\mathrm{OH} \rightarrow \mathrm{CH}_{2} \mathrm{OH}+\mathrm{H}_{2} \mathrm{O}
$$

Das durch das $\mathrm{OH}$-Radikal entstandene $\mathrm{CH}_{2} \mathrm{OH}$ wird mit Hilfe eines H-Radikals in Formaldehyd $\mathrm{CH}_{2} \mathrm{O}$ umgewandelt.

$$
\mathrm{CH}_{2} \mathrm{OH}+\mathrm{H} \rightarrow \mathrm{CH}_{2} \mathrm{O}+\mathrm{H}_{2}
$$

Von diesem stabilen Formaldehyd-Molekül wird ein weiteres Wasserstoffatom abgespalten. Dies kann entweder durch ein H- oder ein OH-Radikal abstrahiert werden.

$$
\begin{gathered}
\mathrm{CH}_{2} \mathrm{O}+\mathrm{H} \rightarrow \mathrm{CHO}+\mathrm{H}_{2} \\
\mathrm{CH}_{2} \mathrm{O}+\mathrm{OH} \rightarrow \mathrm{CHO}+\mathrm{H}_{2} \mathrm{O}
\end{gathered}
$$

Eine Überführung des entstandenen CHO-Radikals in das stabile Zwischenprodukt $\mathrm{CO}$ entsteht durch die Reaktion mit Radikalen oder inerten Stoßpartnern M.

$$
\mathrm{CHO}+\mathrm{H} \rightarrow \mathrm{CO}+\mathrm{H}_{2}
$$




$$
\mathrm{CHO}+\mathrm{M} \rightarrow \mathrm{CO}+\mathrm{H}+\mathrm{M}
$$

Interessant ist hierbei, dass Formaldehyd als Zwischenprodukt während des Oxidationsvorgangs entsteht. Innerhalb diverser Kettenfortpflanzungsreaktionen, wie es auch die Reaktion R13 ist, tritt Formaldehyd in Erscheinung. Bei NiedertemperaturOxidationen handelt es sich um Reaktionen, die in Serienmotoren vorkommen und bei Temperaturen im Bereich von 800 bis 900K aufgrund von Wandwärmeverlusten auftreten. Unter diesen Umständen können lokale Zündzentren, die in Kapitel 3.1.2 beschriebenen hot spots, auftreten und eine Selbstzündung auslösen. Dabei entsteht Formaldehyd im Endgas vor der Flammenfront und nach der Selbstzündung verschwindet dieses Zwischenprodukt wieder durch die vollständige Verbrennung. Dies nutzte Bäuerle et al. [22] und brachte den experimentellen Nachweis von hot spots. Dieser Umstand macht das spektroskopisch gut nachweisbare Molekül Formaldehyd zu einer geeigneten Tracersubstanz für das spektroskopische Verfahren zur Temperaturbestimmung.

Die Reaktionskinetik verdeutlicht durch die Beschreibung der einzelnen Elementarreaktionen recht gut die Bedeutung der Temperatur für die Verbrennung. Der Ablauf einer Reaktion wird durch die sogenannte Reaktionskonstante bzw. den Geschwindigkeitskoeffizienten $k$ beschrieben.

$$
\mathrm{A}+\mathrm{B} \stackrel{\mathrm{k}}{\rightarrow} \mathrm{C}+\mathrm{D}
$$

Ob eine Reaktion der Edukte A und B zu den Produkten C und D stattfindet, hängt von diesem Geschwindigkeitskoeffizienten ab und kann mit dem Arrhenius-Ansatz

$$
k=A \cdot \exp \left(-\frac{E_{a}}{R T}\right)
$$

beschrieben werden. $A$ ist der präexponentielle Faktor, $E_{a}$ die Energieschwelle, die zum Ablauf der Reaktion überwunden werden muss. $R$ bildet die molare Gaskonstante und $T$ die Temperatur.

Um eine Verbrennung zu optimieren, kommt man um die Gesetze der Reaktionskinetik nicht herum, welche aber über die Temperatur beinflussbar sind.

\subsection{Eigenschaften des Formaldehyds}

Formaldehyd ist ein vieratomiges, nichtlineares Molekül mit der Summenformel $\mathrm{CH}_{2} \mathrm{O}$ und der IUPAC-Name ist Methanal. Formaldehyd ${ }^{1}$ ist das einfachste Aldehyd, welches eine Carbonyl-Gruppe CHO enthält. Unter Normalbedingungen ist

\footnotetext{
${ }^{1}$ Der Trivialname des Aldehyds leitet sich von lat. formica, Ameise ab, da Methanal durch Oxidation in Ameisensäure (Methansäure) überführt werden kann.
} 
Formaldehyd gasförmig, wird als stechender Geruch wahrgenommen und reizt die Schleimhäute [23].

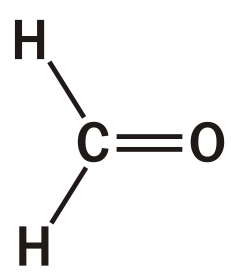

Abbildung 4.4: Strukturformel des Formaldehyds. Im Grundzustand bilden die CH-Verbindungen einen Winkel von 116,18 . Die Abstände der Verbindungen sind nach [24] für die CH-Verbindungen $r_{C H}=$ $1,1005 \cdot 10^{-10} \mathrm{~m}$ und für die Doppelbindung $r_{C O}=1,2033 \cdot 10^{-10} \mathrm{~m}$.

Formaldehyd ist ein Zwischenprodukt bei der Verbrennung und entsteht durch Oxidation von Kohlenwasserstoffen. Es wird hauptsächlich durch das Methylradikal $\mathrm{CH}_{3}$ gebildet und durch Reaktion mit $\mathrm{H}, \mathrm{O}$ und $\mathrm{OH}$ über das Formylradikal $\mathrm{CHO} z u$ Kohlenmonoxid CO abgebaut. Typischerweise tritt Formaldehyd in der sogenannten Vorreaktionszone auf und wird bei der Zündung in der Flammenfront wieder abgebaut. Ein weiterer interessanter Aspekt ist, dass bei der motorischen Kompression des Gemisches eine Temperatur- und Druckerhöhung entsteht, welche die chemische Vorreaktion beschleunigt, und somit verstärkt Formaldehyd gebildet wird. Aufgrund dieser Tatsachen wurden einige LIF-Untersuchungen an der Flamme durchgeführt [25, 26, 27, 28, 29]. Die Detektion von Formaldehyd leistet einen entscheidenden Beitrag zur Entschlüsselung der Reaktionskinetik der Verbrennung und ist somit ein geeignetes Tracer-Molekül für die Verbrennungsdiagnostik.

Die spektroskopischen Eigenschaften des Formaldehyds stellen gute Bedingungen für die quantenmechanische Analyse dieses Moleküls dar. Daher ist Formaldehyd eines der größten Moleküle, bei dem das UV-Absorptionsspektrum detailliert aufgeklärt werden konnte [30]. Die Ursache für diese guten Bedingungen ist zum einen die Tatsache, dass $\mathrm{CH}_{2} \mathrm{O}$ in seinem Grundzustand sehr stabil ist [31], was zur Folge hat, dass die Linienbreiten der rotatorischen Übergänge extrem klein sind und bei kleinen Drücken nur durch die natürliche Doppler-Verbreiterung begrenzt sind. Die zu Vibrationsbändern überlappenden Rotationslinien können gerade noch so weit spektral getrennt werden, dass bei Atmosphärendruck noch die Rotationsstruktur aufgelöst werden kann [32, 27]. Dieser Sachverhalt ist bei einem vieratomigen Molekül überraschend. Eine gute Aufklärung des Vibrationsspektrums liefern die sechs Schwingungsfreiheitsgrade. Diese Vibrationsmoden sind in Abbildung 4.5 dargestellt und die dazugehörigen Frequenzen in Tabelle 4.1 aufgelistet. 


\begin{tabular}{|c|c|c|c|}
\hline Vibration & Bezeichnung & {$\left[\mathrm{cm}^{-1}\right]$} & {$\left[\mathrm{cm}^{-1}\right]$} \\
& $($ Symmetrie $)$ & $\tilde{\mathrm{X}}^{1} \mathrm{~A}_{1}$ & $\tilde{\mathrm{A}}^{1} \mathrm{~A}_{2}$ \\
\hline Symmetrische C-H-Streckschwingung & $\nu_{1}\left(a_{1}\right)$ & 2728,5 & 2846 \\
C-O-Streckschwingung & $\nu_{2}\left(a_{1}\right)$ & 1746,5 & 1183 \\
Symmetrische $\mathrm{CH}_{2}$-Biegeschwingung & $\nu_{3}\left(a_{1}\right)$ & 1500,2 & 1293,1 \\
Biegeschwingung aus der Ebene & $\nu_{4}\left(b_{1}\right)$ & 1167,3 & 1429,3 \\
Antisymmetrische C-H-Streckschwingung & $\nu_{5}\left(b_{2}\right)$ & 2843,3 & 2968,3 \\
$\mathrm{CH}_{2}$-Verdrehung & $\nu_{6}\left(b_{2}\right)$ & 1249,1 & 904 \\
\hline
\end{tabular}

Tabelle 4.1: Frequenzen der sechs Schwingungsmoden im elektronischen Grundzustand $\tilde{\mathrm{X}}^{1} \mathrm{~A}_{1}$ und im angeregten Zustand $\tilde{\mathrm{A}}^{1} \mathrm{~A}_{2}[24]$.
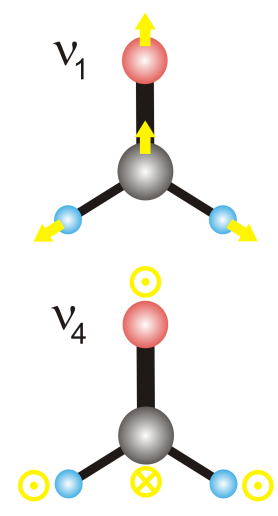
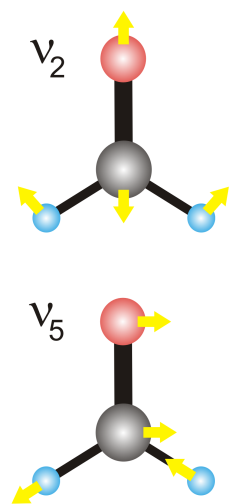
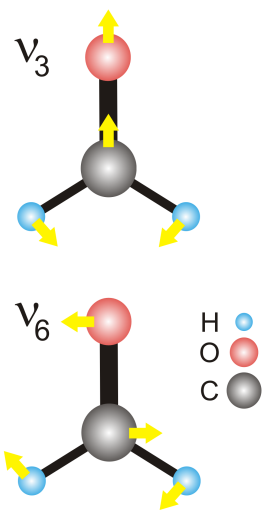

Abbildung 4.5: Sechs Eigenmoden von Formaldehyd nach Herzberg [33]. Die CODoppelbindung ist durch eine größere Strichstärke verdeutlicht.

Im Grundzustand $\left(\tilde{\mathrm{X}}^{1} \mathrm{~A}_{1}\right)$ ist das Molekül planar und hat eine Y-Form, wobei die $\mathrm{C}=\mathrm{O}$-Bindung die Symmetrieachse bildet (siehe Abbildung 4.4). Eine Rotation kann um diese Doppelbindung zwischen dem Sauerstoff- und dem Kohlenstoffatom erfolgen, wo sich auch nach den Daten von Clouthier [24] der Schwerpunkt des Moleküls befindet. Des Weiteren gibt es zwei Rotationsfreiheitsgrade senkrecht zur $\mathrm{C}=\mathrm{O}$-Bindung. Aufgrund des hohen Trägheitsmoments, vor allem senkrecht zur Doppelbindung, entsteht ein geringer Abstand der benachbarten Rotationslinien, so dass bei höheren Drücken keine linienhafte Rotationsstruktur aufgrund des starken Überlapps mehr vorhanden ist. Das UV-Bandenspektrum der Absoption resultiert aus kombinierten elektronischen und vibronischen Übergängen, bei denen die Rotationsstruktur aufgeprägt ist [31]. Die ausgeprägte Bandenstruktur des Absorptionsspektrums im nahen UV ist trotz des komplizierten Aufbaus, wie schon erwähnt, gut aufgeklärt. Abbildung 4.6 zeigt dieses Spektrum. 


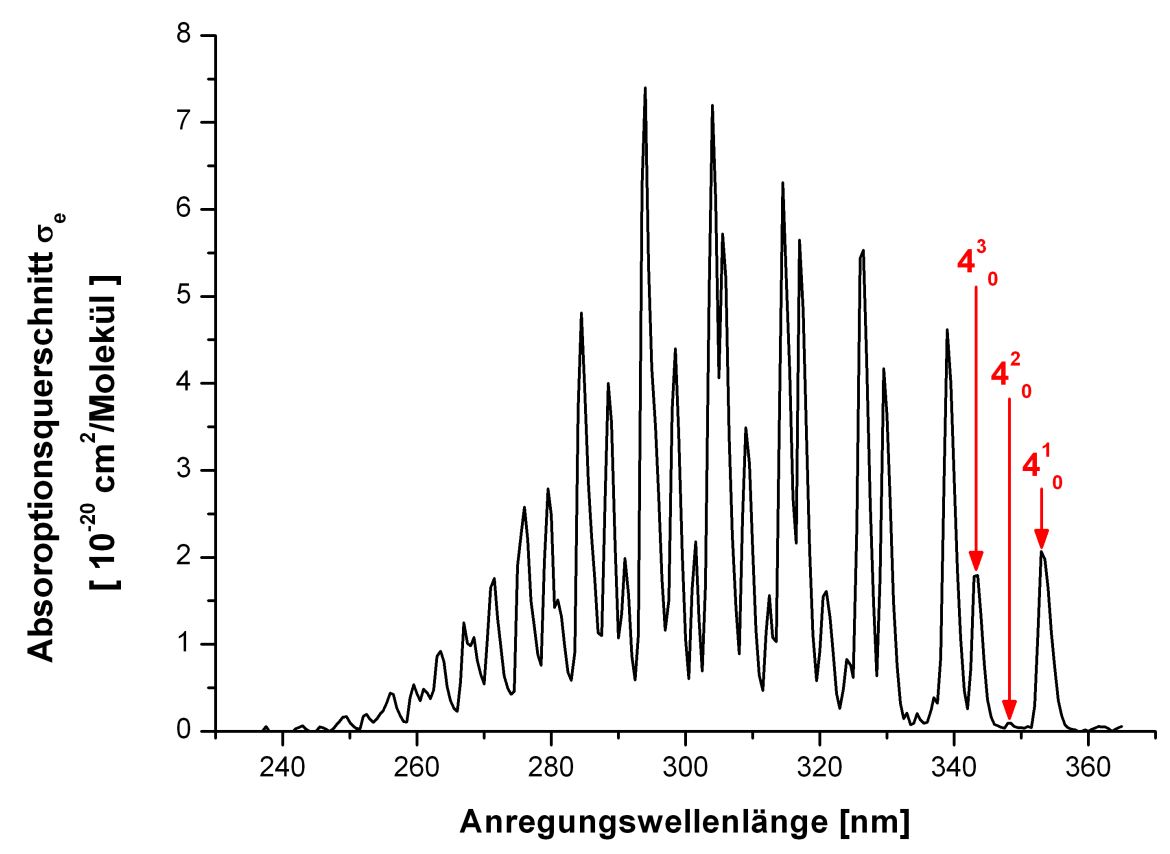

Abbildung 4.6: Absorptionsspektrum von Formaldehyd. Die Schwingungsbande $4_{0}^{1}$ entsteht durch den kombinierten Übergang $\mathrm{A}^{1} \mathrm{~A}_{2} \rightarrow \mathrm{X}^{1} \mathrm{~A}_{1}$. Für diesen $2_{0}^{n} 4_{0}^{1}$-Übergang ist die Schwingungsquantenzahl $n=0$. (Datenquelle: [34])

Das Emissionsspektrum der Fluoreszenz des Formaldehyds weist ebenfalls eine ausgeprägte Vibrationsbandenstruktur im Wellenlängenbereich von 380 bis 520nm auf. In Abbildung 4.7 ist dieses Emissionsspektrum zu sehen.

Für eine genaue Beschreibung der optischen Übergänge wird eine Nomenklatur mit folgender Grundstruktur verwendet:

$$
n_{v^{\prime \prime}}^{v^{\prime}}
$$

Der Parameter $n$ beschreibt den Index $i$ der Fundamentalschwingung $\nu_{i}$, während $v^{\prime \prime}$ und $v^{\prime}$ die Anzahl der angeregten Quanten der entsprechenden Schwingung im unteren und oberen elektronischen Zustand darstellt. Bei Kombinationsschwingungen werden mehrere Symbole einfach aneinander gereiht. In den Spektren in Abbildung 4.6 und 4.7 sind solche Übergänge zu erkennen. Das Emissionsspektrum zeigt dabei Übergänge von Kombinationsschwingungen. 


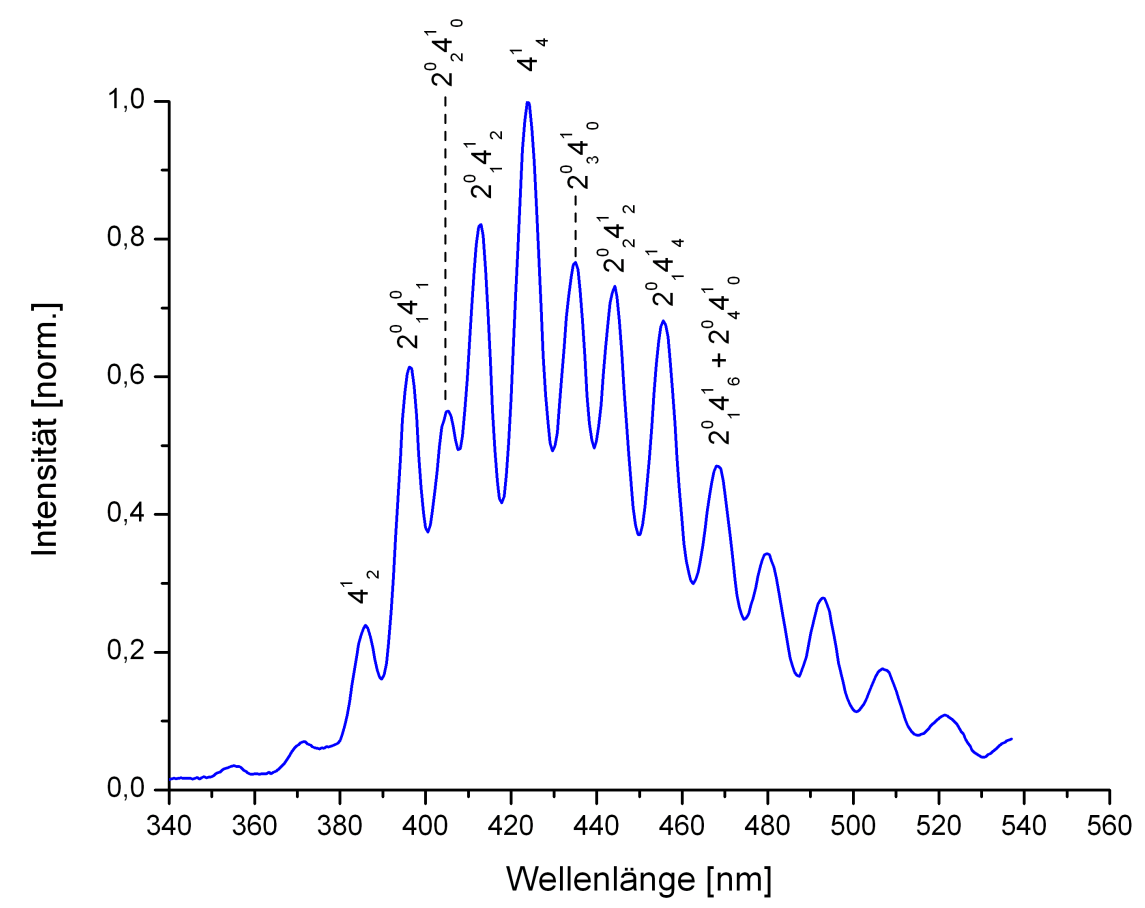

Abbildung 4.7: Gemessenes Emissionsspektrum von Formaldehyd (gemessen mit dem Laserfluorimeter der Umweltgruppe am Laser-Laboratorium Göttingen). Eingezeichnet sind einige Übergänge, die auch für diese Arbeit verwendet wurden (siehe Kapitel 6.2).

\subsubsection{Verfahrenspezifische Eigenschaften}

Neben der Tatsache, dass Formaldehyd ein Bestandteil im Verbrennungsprozess ist, besitzt diese Substanz spektroskopische Eigenschaften, die einen positiven Einfluss auf das hier vorgestellte Verfahren haben. Der in Abbildung 4.6 markierte Übergang $4_{0}^{1}$ hat den Vorteil, dass dieser experimentell recht einfach angeregt werden kann. Brackmann et al. [35] untersuchten mit Hilfe eines frequenzverdreifachten Nd:YAG-Lasers (Laserwellenlänge 355nm) die Anregung von Formaldehyd auch unter motorischen Bedingungen mit Erfolg. Wie das Absorptionsspektrum zeigt, ist dieser Übergang eher schwach gegenüber den anderen Übergängen. Dies hat aber den Vorteil, dass keine Sättigung der Fluoreszenz trotz der hohen Energieleistungen eines Nd:YAG-Lasers eintritt.

Für das in dieser Arbeit vorgestellte Temperaturmessverfahren muss die Bedingung erfüllt sein, das die Thermalisierung des Moleküls im angeregten Zustand innerhalb der Fluoreszenzlebensdauer abgeschlossen ist. Hier haben die strahlungslosen Konkurrenzprozesse einen entscheidenden Einfluss und wurden von Weisshaar et al. [36] für kleine Drücke untersucht. Metz et al. [37] untersuchten diese spektroskopischen 
Größen unter motorrelevanten Bedingungen und erhielten Fluoreszenzlebensdauern von 33 bis $25 \mathrm{~ns}$ bei einer Temperatur von $295 \mathrm{~K}$ und Drücken von 1 bis 5 bar und bei einer Temperatur von $770 \mathrm{~K}$ Lebendauern zwischen 9 und 5,9 ns. Des Weiteren ermittelten sie eine Thermalisierungszeit von 7,5 bis 120 ps. Diese Resultate erfüllen die oben genannten notwendigen Bedingungen, um eine Temperaturinformation von Formaldehyd zu erhalten.

Die für das Verfahren untersuchten Übergänge befinden sich im Wellenlängenbereich von 390 bis $420 \mathrm{~nm}$ und sind Kombinationsübergänge der beiden Fundamentalschwingungen $\nu_{2}$ und $\nu_{4}$. Gemäß der Nomenklaturregel 4.20 sind dies $2_{1}^{0} 4_{1}^{0}(395 \mathrm{~nm})$, $4_{3}^{0}(405 \mathrm{~nm})$ und $2_{1}^{0} 4_{2}^{1}(413 \mathrm{~nm})$. Die Besetzungen der angeregten Zustände dieser Übergänge wird mit Hilfe der Fluoreszenzintensität erfasst. Mit Hilfe der Gleichung 4.10 ergibt sich die Bildung des folgenden Verhältnisses

$$
\frac{F_{1}}{F_{2}}=\frac{c \cdot I_{1} \cdot V \cdot \sigma_{1} \cdot \Phi_{1} \cdot N_{0} \cdot e^{-\frac{E_{1}}{k_{B} T}}}{c \cdot I_{2} \cdot V \cdot \sigma_{2} \cdot \Phi_{2} \cdot N_{0} \cdot e^{-\frac{E_{2}}{k_{B} T}}}
$$

Da bei diesem Verfahren mit nur einem Laser angeregt wird gilt $I_{1}=I_{2}$. Des Weiteren wird aufgrund der Ein-Laser-Anregung in einen höheren Zustand des Moleküls angeregt, was zur Folge hat, dass nur ein Absorptionskoeffizient vorliegt und somit $\sigma_{1}=\sigma_{2}$ ist. Dadurch vereinfacht sich Gleichung $4.21 \mathrm{zu}$

$$
\frac{F_{1}}{F_{2}}=\frac{\Phi_{1} \cdot e^{-\frac{E_{1}}{k_{B} T}}}{\Phi_{2} \cdot e^{-\frac{E_{2}}{k_{B} T}}}
$$

Für die Temperaturmessung basierend auf dem Intensitätsverhältnis muss das Verhältnis der Quantenausbeuten konstant sein. Die Quantenausbeuten sind dabei von den Reaktionskonstanten des strahlungsaktiven Prozesses $k_{r a d}$, dem strahlungslosen Prozess $k_{n r a d}$, der Fluoreszenzlöschung $k_{Q i}$ und dem Partialdruck $p_{i}$ der Komponente $i$ abhängig.

$$
\Phi=\frac{k_{\text {rad }}}{k_{\text {gesamt }}}=\frac{k_{\text {rad }}}{k_{\text {rad }}+k_{n r a d}+\sum_{i} k_{Q_{i}} \cdot p_{i}}=\frac{k_{\text {rad }}}{k_{s}+\sum_{i} k_{Q_{i}} \cdot p_{i}}
$$

Nach Burkert et al. [38] gilt für die Anregung das Verhältnis der Quantenausbeuten der Zustände $4^{1}$ und $4^{0}$ des Formaldehyds

$$
\frac{\Phi\left(4^{1}\right)}{\Phi\left(4^{0}\right)}=\frac{k_{\text {rad }}\left(4^{1}\right)}{k_{\text {rad }}\left(4^{0}\right)} \cdot \frac{k_{Q M}\left(4^{1}\right) \cdot\left[p_{M}\right]+k_{Q p_{i}}\left(4^{1}\right) \cdot\left[p_{p_{i}}\right]+\ldots+k_{s}\left(4^{1}\right)}{k_{Q M}\left(4^{0}\right) \cdot\left[p_{M}\right]+k_{Q p_{i}}\left(4^{0}\right) \cdot\left[p_{p_{i}}\right]+\ldots+k_{s}\left(4^{0}\right)}=\text { konst } .
$$

Die Indizes $M$ stehen für Formaldehyd und $P_{i}$ für das Puffergas $i$. Shibuya et al. [39], [40] haben im Rahmen der Messgenauigkeit folgende Werte ermittelt: 


$$
\begin{aligned}
\frac{k_{r a d}\left(4^{1}\right)}{k_{r a d}\left(4^{0}\right)} & =1,48 \pm 0,27 \\
k_{s}\left(4^{1}\right)=k_{s}\left(4^{0}\right) & =1,22 \cdot 10^{7} s^{-1}
\end{aligned}
$$

Die Untersuchungen von Shibuya zeigten auch, dass von den folgenden Relationen $k_{Q M}\left(4^{0}\right)=k_{Q M}\left(4^{1}\right)$ und $k_{Q p_{i}}\left(4^{0}\right)=k_{Q p_{i}}\left(4^{1}\right)$ ausgegangen werden kann. Das bedeutet, dass beide Fluoreszenzquantenausbeuten $\Phi\left(4^{0}\right)$ und $\Phi\left(4^{1}\right)$ in gleicher Weise von den Gaszusammensetzungen abhängen und damit das Verhältnis von dieser Zusammensetzung unabhängig ist. Durch Einsetzen der folgenden Teilchenzahlen $N_{1}$ und $N_{2}$ mit

$$
\begin{aligned}
& N_{1}=N_{0} \cdot e^{-\frac{E_{1}}{k_{B} T}} \\
& N_{2}=N_{0} \cdot e^{-\frac{E_{2}}{k_{B} T}}
\end{aligned}
$$

in Gleichung 4.21 erhält man die temperaturabhängige Arrhenius-Funktion

$$
f(T)=\frac{F_{1}}{F_{2}}=\frac{N_{1}}{N_{2}}=e^{-\frac{\Delta E}{k_{B} T}} .
$$




\section{Kapitel 5}

\section{Technologische Grundlagen des angewandten Verfahrens}

Dieses Kapitel gibt einen Überblick über angewandte technische Komponenten, die zur Realisierung des vorgestellten Messverfahrens herangezogen werden. Ziel der technischen Umsetzung ist es, ein minimal invasives Sensorsystem zu entwickeln. Eine Miniaturisierung gewährleistet nahezu unveränderte physikalische Eigenschaften des zu untersuchenden Motors. Mit einem solchen Verfahren können Temperaturwerte erfasst werden, die auch in Serienmotoren vorherrschen. Generell kann das angepasste Sensorsytem für gering modifizierte Motoren Verwendung finden. Somit entfällt ein komplexer und kostspieliger Umbau zu einem speziellen Forschungsmotor. Weiterhin wird durch minimale Abmessung eine hohe Mobilität des Messverfahrens ermöglicht.

Da es sich bei der motorischen Applikation um eine Technik handelt, bei der starke Schwingungen und ein fluktuierendes Milieu im Brennraum die Messungen erschweren, müssen diverse kontruktive Abweichung vom standardisierten Laboraufbau vollzogen werden. Die zu dieser Anpassung verwendeten optischen Bauelemente und Erfassungssysteme werden in diesem Kapitel besprochen. Zusätzlich werden die heutigen wissenschaftlichen Methoden zur Motorenforschung dargestellt. Zum Schluß wird das in dieser Arbeit entwickelte Sensorsytem vorgestellt.

\subsection{Faseroptik}

Die optische Faser besteht im wesentlichen aus einen hochtransparenten Kern mit der Brechzahl $n_{K}$ (engl. „core“) und einem ihn umgebenden Mantel mit einer Brechzahl $n_{M}$ (engl. „cladding“"). Abbildung 5.1 zeigt den prinzipiellen Aufbau einer Faser.

Das Signal wird durch mehrfache Totalreflexion zwischen den Grenzflächen des Kern- und Mantelmaterials durch die Faser geführt. Der entscheidende Vorteil ist, dass bei einer gekrümmten Faser das Licht dieser Krümmung folgt. Um die Bedingungen für eine Totalreflexion zu erfüllen, muss das Kernmaterial eine größere Brechzahl haben als das Mantelmaterial, es gilt also $n_{K}>n_{M}$. Beim Übergang eines Lichtstrahles von einem optisch dichten (größere Brechzahl $n_{1}$ ) in ein optisch 


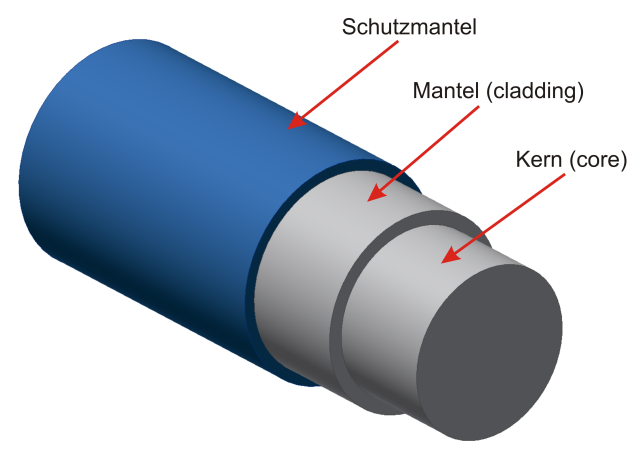

Abbildung 5.1: Struktur der Faser mit einem äußeren Schutzmantel. Das Kernmaterial hat einen höheren Brechungsindex als das Mantelmaterial.

dünneres Medium (kleinere Brechzahl $n_{2}$ ) wird dieser vom Lot weggebrochen (siehe Abbildung 5.2). Der Ausfallswinkel $\alpha_{2}$ ist somit größer als der Einfallswinkel $\alpha_{1}$. Dieses Phänomen wird durch das Snelliussche Brechungsgesetz beschrieben:

$$
n_{1} \cdot \sin \alpha_{1}=n_{2} \cdot \sin \alpha_{2}
$$

Wird der Einfallswinkel kontinuierlich vergrößert, tritt bei einem Winkel $\alpha_{t}$ das Licht nicht mehr in das dünnere Medium ein, sondern verläuft entlang der Grenzschicht zwischen den beiden Medien. Bei einer weiteren Vergrößerung des Winkels wird der Lichtstrahl an der Grenzfläche total reflektiert und verläßt das Medium nicht mehr. Bei dem Grenzfall mit dem Einfallswinkel $\alpha_{t}$ wäre der Ausfallswinkel $\alpha_{t g}=90^{\circ}$. Für den Grenzwinkel der Totalreflexion gilt somit

$$
\sin \alpha_{t}=\frac{n_{2}}{n_{1}}
$$

Der Grenzwinkel der Totalreflexion innerhalb einer Faser ist somit von den Brechungsindizes des Kern- und Mantelmaterials abhängig.

$$
\sin \alpha_{t}=\frac{n_{M}}{n_{K}}
$$

Aufgrund des Winkels $\alpha_{t}$ ergibt sich ein maximaler Einstrahlwinkel für die in eine Faser einzukoppelnden Lichtstrahlen, welche im Kern durch Totalreflexion geführt werden können. Dieser faserspezifische Öffnungswinkel wird auch Akzeptanzwinkel $\Theta_{\max }$ genannt und ergibt sich durch Anwendung des Brechungsgesetzes an der Einkoppelfläche und durch Einsetzen von $\alpha_{t}$ :

$$
\sin \Theta_{\max }=\frac{1}{n_{0}} \cdot \sqrt{n_{K}^{2}-n_{M}^{2}}
$$

Wobei $n_{0}$ die Brechzahl des Mediums ist, aus dem der Lichtstrahl in die Faser eintritt. In den meisten Fällen ist dies Luft $\left(n_{0}=1\right)$. Man spricht hierbei auch von 


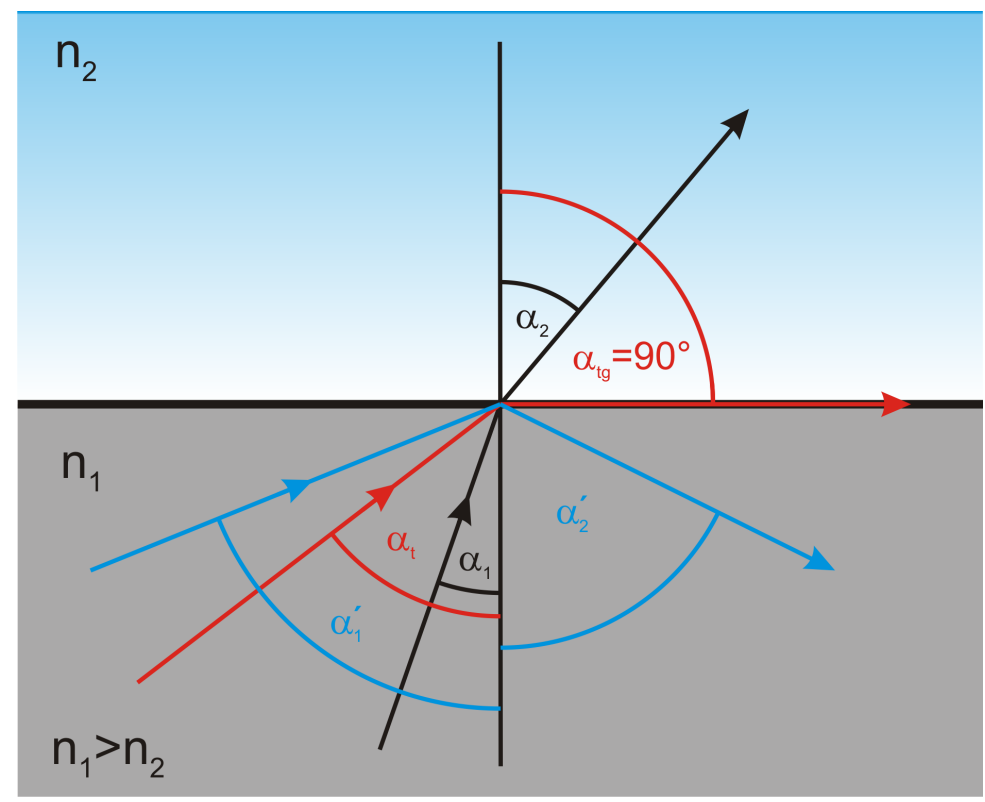

Abbildung 5.2: Bedingungen für den Übergang von einem optisch dichteren zu einem dünneren Medium. Bei allen Einfallswinkeln, die größer als der Grenzwinkel $\alpha_{t}$ sind, entsteht Totalreflexion.

der Numerischen Apertur NA, welche ein qualitatives Maß für die Fähigkeit einer Faser ist, Licht zu sammeln.

$$
N A=n_{0} \cdot \sin \Theta_{\max } \quad \text { oder für Luftumgebung } \quad N A=\sin \Theta_{\max }
$$

Mit Gleichung 5.4 wird ersichtlich, dass die numerische Apertur von Brechzahldifferenz des Kern- und Mantelmaterials abhängt. Durch eine Dotierung des Kernmaterials kann der Brechungsindex im Vergleich zum Mantelmaterial erhöht werden, was wiederum die numerische Apertur vergrößert. Zu beachten ist allerdings, dass aufgrund des Dotierungsmaterials höhere Streuverluste einhergehen. Eine Quarzglasfaser hat zum Beispiel einen NA-Standardwert von 0,22. Sogenannte Low-NA-Fasern liegen im Bereich von 0,1-0,12 und High-NA-Fasern haben eine NA von 0,28-0,3 ${ }^{1}$. Mit den oben geschilderten Gesetzen der geometrischen Optik kann die Lichtausbreitung in Lichtleitern gut beschrieben werden, dessen Durchmesser groß im Vergleich zur Wellenlänge des zu führenden Lichtes ist. Befindet sich der Durchmesser des Lichtleiters hingegen in der Größenordnung der Lichtwellenlänge, so muss der Wellencharakter des Lichtes berücksichtigt werden und die Ausbreitung verhält sich analog zur Ausbreitung von Mikrowellen in Wellenleitern. Deshalb wird auch von Lichtwellenleitern (LWL) gesprochen.

Unter diesen Bedingung werden nur Wellenfronten im Lichtleiter geführt, die sich

\footnotetext{
${ }^{1}$ Faserdaten der Firma CeramOptec
} 

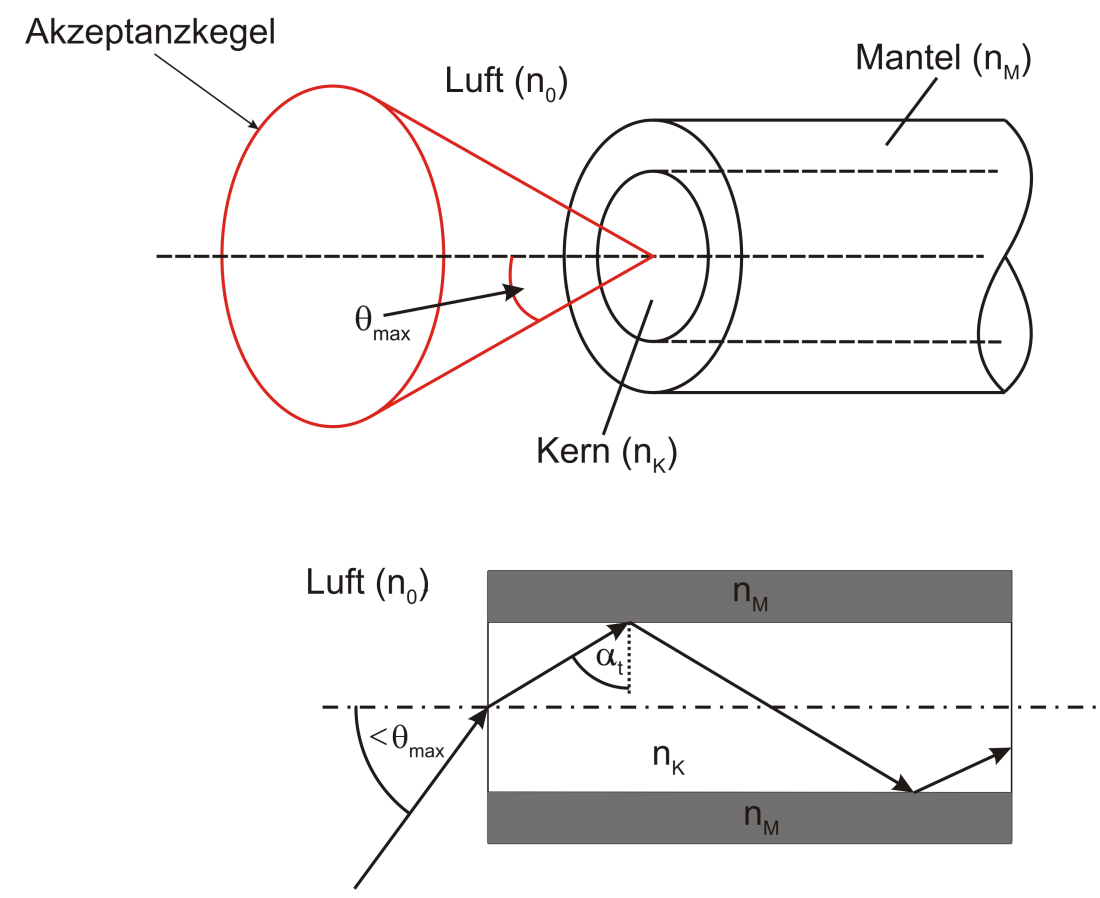

Abbildung 5.3: Bedingungen für die Lichteinkopplung in Fasern (hier in Luftumgebung). Die Lichtstrahlen müssen innerhalb des Akzeptanzkegels auftreffen, damit diese auch innerhalb des Grenzwinkels $\alpha_{t}$ der Totalreflexion verlaufen.

nach der Reflexion an der Grenzfläche konstruktiv überlagern. Eine elektromagnetische Welle, die diese Bedingungen erfüllt, wird auch als Mode bezeichnet. Eine Betrachtung von Moden hat starken Einfluss auf die Lichtleiterstruktur. Die eingangs beschriebene Struktur einer Faser besteht aus einem Kernmaterial mit einer konstanten Brechzahl $n_{K}$ und ist ummantelt mit einem Material, welches ebenfalls eine konstante Brechzahl $n_{M}$ besitzt. Man spricht hier von einer Stufen-Index-Faser, da sich der Brechungsindex von innen nach außen stufenförmig ändert. Bei diesem Fasertyp entstehen unterschiedliche Laufzeiten der einzelnen Moden, da achsnahe Moden (auch als low-order-modes oder axiale Moden bezeichnet) weniger reflektiert werden als die sogenannten high-order-modes. Abbildung 5.4 verdeutlicht dieses Phänomen. Aufgrund dieser Modendispersion verschmieren Signale auf langen Übertragungsstrecken. Das läßt sich verhindern, indem man sogenannte GradientenIndex-Fasern verwendet. Hierbei liegt kein konstanter Brechungsindex innerhalb des Kernmaterials vor. Dieser nimmt von innen nach außen kontinuierlich ab. Dadurch verlaufen die Strahlen in leicht gekrümmten Bahnen um die Längsachse der Faser. Aufgrund des höheren Brechungsindexes breiten sich die achsnahen Strahlen langsamer aus. Somit werden die unterschiedlichen Laufzeiten der verschiedenen Moden ausgeglichen. 


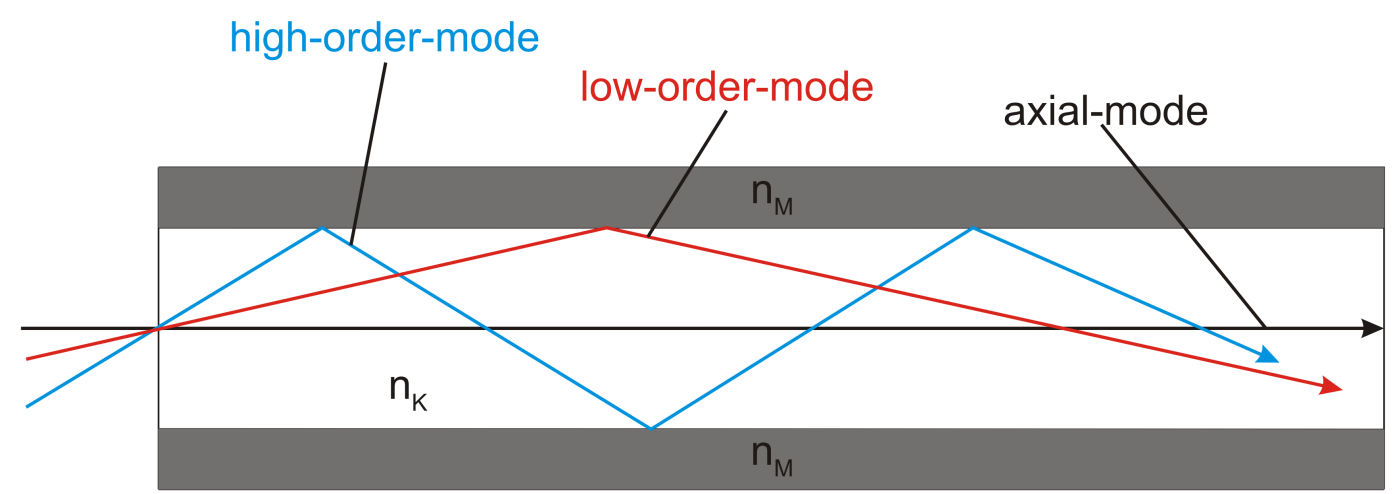

Abbildung 5.4: Unterschiedliche Laufzeiten der Moden durch achsnahen Verlauf (wenig Reflexionen) gegenüber den häufig reflektierten Moden (highorder-mode).

Eine weitere Möglichkeit zur Verhinderung von Modendispersionen ist der Einsatz von sogenannten Single-Mode-Fasern. Der Unterschied zu den oben beschriebenen Multi-Mode-Fasern ist ihr sehr kleiner Kerndurchmesser, so dass sich nur eine Mode ausbreiten kann. In Abbildung 5.5 sind diese drei Fasertypen schematisch dargestellt. Die Anzahl $N$ der geführten Moden in einer Faser können näherungsweise

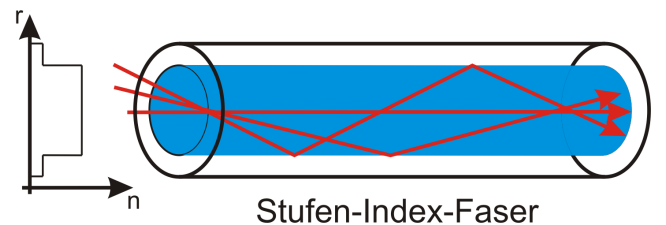

Stufen-Index-Faser
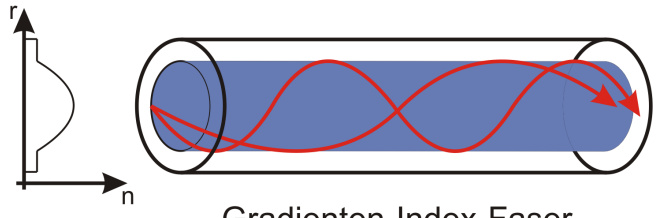

Gradienten-Index-Faser

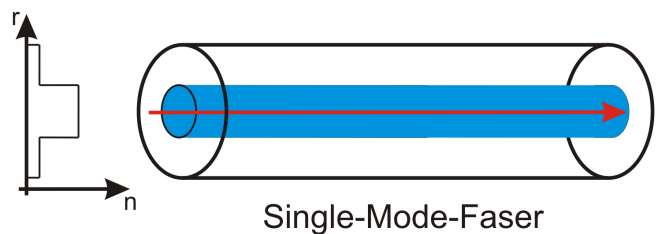

Single-Mode-Faser

Abbildung 5.5: Lichtführung in den verschiedenen Fasertypen mit unterschiedlichen Brechzahlprofilen.

durch

$$
N \approx \frac{1}{2} \cdot \frac{g}{g+2} \cdot V^{2}
$$

beschrieben werden [41]. Dabei beschreibt der Profilexponent $g$ die Brechzahlverteilung innerhalb der Faser und $V$ ist die sogenannte Faserkenngröße zur Bestimmung der Modenzahl (auch als normierte Frequenz bekannt). Letztere ist abhängig vom 
Kernradius $a$, der Wellenlänge $\lambda$ des eingekoppelten Lichts, der numerischen Apertur $N A$ und ist definiert als

$$
V=2 \pi \cdot \frac{a}{\lambda} \cdot N A
$$

Für eine Stufen-Index-Faser gilt $g \rightarrow \infty$ und es ergibt sich für die Modenanzahl $N \approx \frac{1}{2} \cdot V^{2}$. Bei einem parabolischen Profil, wie es bei einer Gradienten-Index-Faser vorliegt, ist $g=2$, womit sich bei diesem Fasertyp eine Modenanzahl von $N \approx \frac{1}{4} \cdot V^{2}$ einstellt.

Neben den Brechungsindexprofilen sind weitere Unterscheidungsmerkmale von Fasern die verwendeten Kern- und Mantelmaterialien. Vor allem in der Datenkommuniktation finden Kunststoff-Fasern wie zum Beispiel Polymerfasern sehr häufig Anwendung. Diese sind kostengünstig zu produzieren und lassen sich relativ leicht bearbeiten. Ein Nachteil dieser Fasern ist ihr geringerer Transmissionsgrad gegenüber Glasfasern. Zur Herstellung von Glasfasern werden reines Quarzglas oder dotierte Gläser verwendet. Reine Quarzglasfasern eignen sich besonders für die Lichtleitung von UV-Licht, da diese die besten Transmissionswerte für diesen Spektralbereich liefern. Dabei ist sowohl der Kern als auch der Mantel aus Quarzglas. Um eine Brechzahldifferenz zu generieren, ist der Mantel zusätzlich mit Fluor dotiert, was gleichzeitig zu höheren Transmissionsverlusten im UV-Bereich führt. Dieser Sachverhalt ist eine Ursache dafür, dass sich Gradienten-Index-Fasern nicht für UV-Anwendungen eignen. Das in eine Faser eingekoppelte Licht wird nur zu einem bestimmten Teil übertragen, da Verluste aufgrund von Streuung und Absorption enstehen. Des Weiteren kann die Transmission eines Lichtleiters durch längere Einwirkung von UV-Licht vermindert werden, oder sogar vollständig undurchlässig werden. Dieser Effekt wird als Solarisation bezeichnet und basiert auf einer molekularen Strukturänderung innerhalb des Glases. Heutige Fasern sind so hergestellt, dass sie für den UV-Bereich „solarisationsarm“ sind. Das heißt, der Prozess wird in der Faser verlangsamt. Die Dämpfung des Lichtsignals kann mit Hilfe des Lambert-Beer'schen Gesetzes beschrieben werden. Ausgehend von einer eingestrahlten Lichtintensität $I_{0}$, eines vom Kernmaterial abhängigen Dämpfungsfaktor $\epsilon$ und der Faserlänge $l$ erhält man

$$
I=I_{0}^{-\epsilon l}
$$

Die Dämpfung einer Faser wird typischerweise logarithmisch dargestellt. Es ergibt sich somit

$$
\epsilon_{d B}=-10 \cdot \log \frac{I}{I_{0}}
$$

für die Dämpfung einer Faser mit der Einheit Dezibel. Das hat den Vorteil, dass sich verschiedene Dämpfungswerte einfach addieren lassen. Üblicherweise wird die Faserdämpfung in $\mathrm{dB} / \mathrm{km}$ angegeben. 


\subsection{Laserstrahlhomogenisierung}

Die Einkopplung von Laserlicht in eine Faseroptik stellt insofern ein Problem dar, dass eine hohe Laserenergie die Faser zerstören kann. Da bei dem in dieser Arbeit verwendeten Lasersystem kein in der Intensität gleichmäßig verteiltes Laserstrahlprofil vorliegt, entstehen sogenannte hot spots. Dabei handelt es sich um Bereiche mit höherer Energiedichte innerhalb des Strahlprofils, was zu einer lokalen Zerstörung des Fasermaterials und zur Unbrauchbarkeit der Faser führen kann. Eine gleichmäßige Lichtverteilung kann mit Hilfe eines Mikrolinsenarrays (LA1) und einer sphärischen Fourierlinse (FL) realisiert und somit die Entstehung von hot spots unterbunden werden [42]. Dieser optische Aufbau wird auch als Homogenisierer bezeichent, wobei man unter einen nicht-abbildenden und einen abbildenden Homogenisierer unterscheidet. Ersterer zeigt Abbildung 5.6. Beim abbildenden Homogenisierer wird ein zweites Linsenarray zwischen dem ersten Array und der Linse eingesetzt. Die

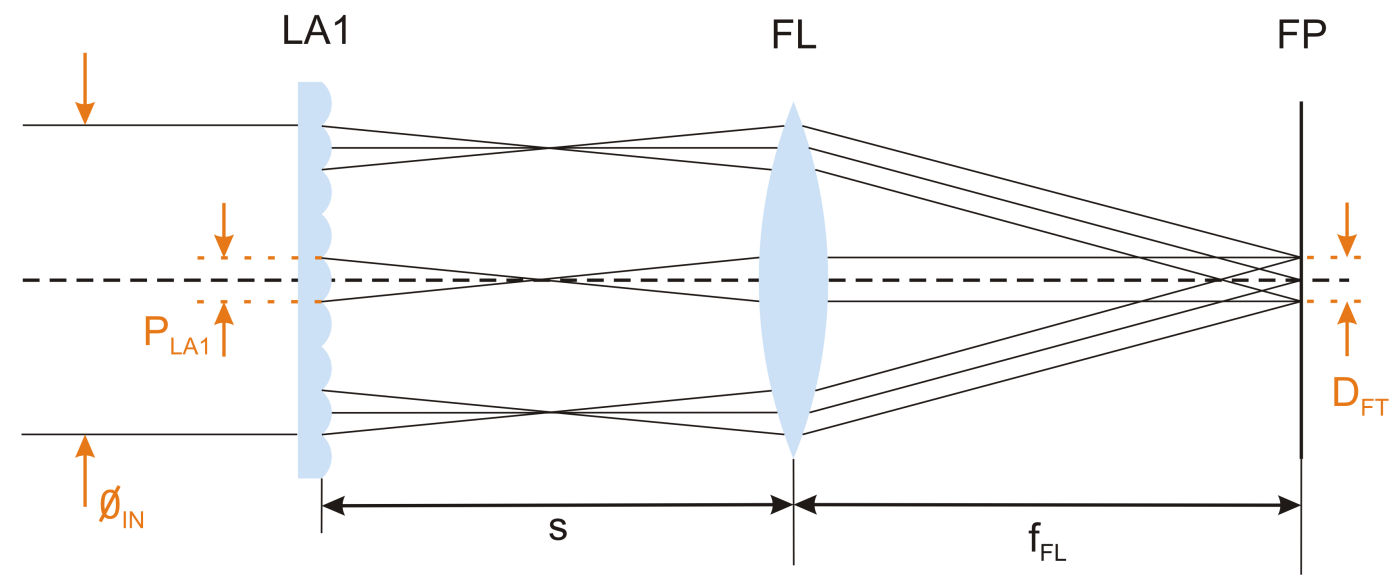

Abbildung 5.6: Strahlhomogenisierung mit Hilfe eines nicht-abbildenden Homogenisators. $\emptyset_{I N}$ ist der Durchmesser des Laserstrahls

Wahl zwischen diesen beiden Varianten hängt stark von der Lichtquelle und der Anwendung ab. Eine Orientierungshilfe bietet hierbei die Fresnelzahl FN, die für Mikrolinsenhomogenisierer definiert ist als

$$
F N \approx \frac{P_{L A 1} \cdot D_{F T}}{4 \cdot \lambda \cdot f_{F L}}
$$

mit $P_{L A 1}$ als Pitch oder Mittenabstand des Mikrolinsenarrays, $D_{F T}$ ist der Durchmesser des Flat-Tops FT (Rechteckprofil), $\lambda$ die Lichtwellenlänge und der Brennweite $f_{F L}$ der Linse FL. Abbildung 5.6 verdeutlicht diese Größen. Nicht-abbildende Homogenisierer weisen häufig starke Beugungseffekte aufgrund der Fresnelbeugung am Mikrolinsenarray auf. Bei Fresnelzahlen FN>10, oder besser FN>100, kommt es 
zu gleichmäßigen Intensitätsverteilungen. Wegen der Proportionalität von Fresnelzahl und Flat-Top eignen sich nicht-abbildende Homogenisierer für die Ausleuchtung großer Flächen. Für kleine Fresnelzahlen $\mathrm{FN}<10$ oder sehr gleichmäßige Verteilungen hingegen ist der abbildende Homogenisierer vorzuziehen.

Das Mikrolinsenarray besteht aus einem Wabenkondensor, dessen Multipupillensystem durch ein Linsenarray realisiert wird. Dieses Array besteht entweder aus gekreuzten Zylinderlinsen oder aus quadratischen Mikrolinsen. Mit der Arrayoptik wird der einfallende Lichtstrahl in eine Vielzahl von mikroskopischen Teilstrahlen zerlegt, welche wiederum mit Hilfe der sphärischen Fourierlinse in deren Brennebene überlagert werden und ein homogen ausgeleuchtetes Feld, das Flat-Top FT, ergeben. Der Durchmesser dieses Flat-Tops ergibt sich mit Hilfe der Brennweite $f_{L A 1}$ des Mikrolinsenarrays aus

$$
D_{F T}=\left|\frac{P_{L A 1} \cdot f_{F L}}{f_{L A}}\right|
$$

In dieser Arbeit wurde ein Mikrolinsenarray von der Firma Suss MicroOptics SA verwendet (CC-Q-300S, 10x10mm), welches folgende Daten besitzt:

- Mittenabstand des Mikrolinsenarrays (Pitch): $P_{L A 1}=0,3 \mathrm{~mm}$

- Brennweite des Linsenarrays: $f_{L A 1}=4,33 \mathrm{~mm}$

Die verwendete Laserwellenlänge beträgt 355nm und die Faser-Einkoppelfläche hat einen Durchmesser von 2,4mm. Letzterer Durchmesser kann mit dem Durchmesser des Flat-Top gleichgesetzt werden, also $D_{F T}=2,4 \mathrm{~mm}$. Mit Gleichung 5.11 ergibt sich damit eine Brennweite $f_{F L}=40 \mathrm{~mm}$ für die Fourierlinse. Durch Einsetzen dieser Komponentendaten in Gleichung 5.10 ergibt sich eine Fresnelzahl von

$$
F N \approx \frac{0,3 \mathrm{~mm} \cdot 2,4 \mathrm{~mm}}{4 \cdot 355 \cdot 10^{-6} \mathrm{~mm} \cdot 40 \mathrm{~mm}} \approx 12,7
$$

Somit wurde für diese Arbeit ein nicht-abbildender Homogenisator für die Lichteinkopplung in die Faser aufgebaut.

\subsection{CCD-Kamera}

Mit Hilfe einer bildverstärkten CCD-Kamera wurde die Detektion des zu analysierenden Fluoreszenzsignals durchgeführt. Kernstück dieses Kameratyps ist das Charge Coupled Device (CCD). Dieser CCD-Sensor besteht aus einer Anordnung von MOS-Kondensatoren (Metall-Elektrode, $\mathrm{SiO}_{2}$ und Siliziumsubstrat), wobei ein auftreffendes Photon in eine elektrische Ladung umwandelt wird. Um das Signal verarbeiten zu können, findet ein Ladungstransport jedes einzelnen Bildpunktes in ein 
Schieberegister statt. Die Information wird von dort über ein Ausleseregister ausgelesen und an den Rechner zur weiteren Verarbeitung weitergeleitet. Neben den Photonen können auch Elektronen im Halbleiterelement aufgrund von thermischen Schwingungen ein Signal erzeugen. Dieses Rauschsignal wird auch als Dunkelstrom bezeichent und erstreckt sich als Dunkelbild über das gesamte Halbleiterelement, welches sich zum Nutzsignal addiert. Abweichend vom Idealfall ist das Dunkelrauschen jedoch nicht gleichmäßig über das Halbleiterelement verteilt, sondern variiert in folge von herstellungsbedingten örtlichen Inhomogenitäten über der Sensorfläche. Um dieses Rauschen zu minimieren, wird das CCD-Element in Kameras generell gekühlt. Da das Dunkelrauschen nicht vollständig eliminiert werden kann, wird ein Dunkelbild aufgenommen und diese feste Dunkelstruktur vom Nutzbild abgezogen. Der Dunkelstrom eines CCD-Bildsensors ist ein wichtiger Einflussfaktor für die Sensitivität $(S)$ einer Kamera, da diese das Verhältnis von Signal-Rauschverhältnis $S / N$ zu Quantenausbeute $Q E$ darstellt.

$$
S=\frac{S / N}{Q E}
$$

Das Verhältnis $S / N$ beinhaltet die Anzahl der Signal-Elektronen $n_{\text {Signal }}$ und die Anzahl der Rausch-Elktronen $n_{\text {noise }}$ wie folgt

$$
\frac{S}{N}=\frac{n_{\text {Signal }}}{n_{\text {noise }}}
$$

Die Quantenausbeute ist ein Maß, das den prozentualen Anteil der aus den einfallenden Photonen gebildeten elektronischen Ladungen angibt

$$
Q E=\frac{n_{\text {Photon }}}{n_{\text {Ladung }}}
$$

Dabei ist $Q E$ spektral keine konstante Größe, sondern von der Wellenlänge abhängig.

Eine bildverstärkte CCD-Kamera besteht aus zwei Baugruppen. Dem oben beschriebenen CCD-Element ist ein Bildverstärker vorgeschaltet, der direkt mit dem CCD verbunden, oder extern mit Hilfe einer Linsenoptik angekoppelt werden kann. Die Verstärkung kommt zustande, indem Photonen innerhalb einer Photokathode weitere Photoelektronen erzeugen. Die Wahrscheinlichkeit dieses Ereignisses ist durch die Quanteneffizienz gegeben. Durch eine geeignete Wahl der Photokathode kann die spektrale Empfindlichkeit einer Kamera entscheidend beeinflusst werden. So wurden in dieser Arbeit bildverstärkte CCD-Kameras verwendet, die mit einer MultialkaliPhotokathode vom Typ S20 ausgestattet sind und somit eine höhere Empfindlichkeit im UV-Bereich gewährleisten. Diese Photokathode wurde von Varma und Hallensleben ([43],[44]) untersucht und charakterisiert. Die neu erzeugten Photoelektronen gelangen direkt in eine Anordnung von nur wenige Mikrometer langen Kanälen, dem 
sogenannten Multichannelplate (MCP). An diesen Kanälen liegt eine einstellbare Spannung an, die eine Beschleunigung der Photoelektronen hervorruft und dadurch abhängig von der angelegten Spannung Sekundärelektronen generiert werden. Damit findet eine Vervielfachung der eintreffenden Elektronen statt. Die Sekundärelektronen treffen wiederum auf einen Phosphorschirm und führen zu Leuchtereignissen, die in ihrer Intensität proportional zum Eingangssignal sind. Mit Hilfe eines Faserbündels ist der CCD-Sensor direkt an den Phosphorschirm gekoppelt und sammelt das emittierte Licht auf (siehe Abbildung 5.7). Bei den in dieser Arbeit verwendeten bildverstärkten Kameras von der Firma LaVision kann die erwähnte Spannung am MCP über ein Potentiometer eingestellt werden. Diese als gain bezeichnete Größe gibt die Empfindlichkeit der Kamera vor.

Will man Signale aus einem Spektralbereich detektieren, der außerhalb der spek-

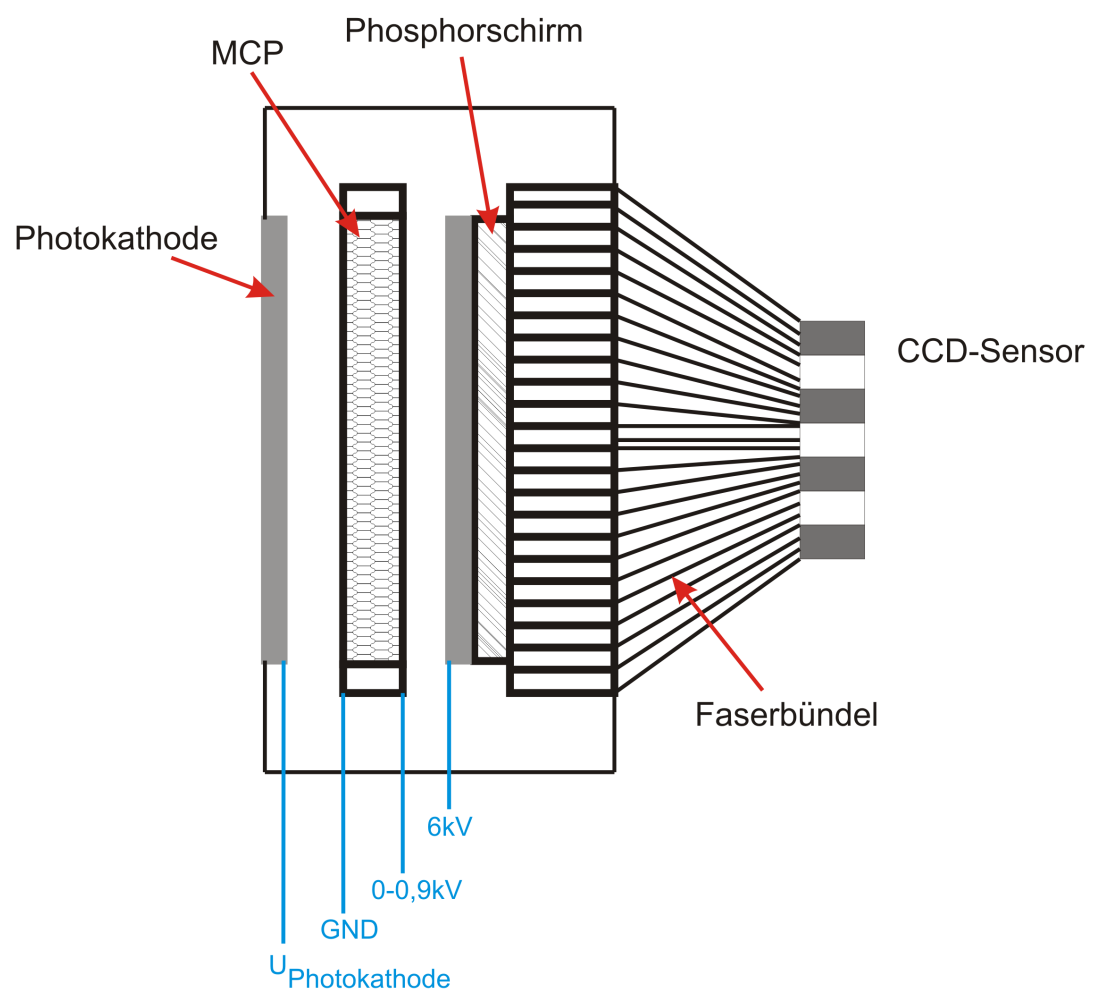

Abbildung 5.7: Schematischer Aufbau einer bildverstärkten CCD-Kamera. Durch Variation der elektrischen Spannung am MCP (hier: 0-0,9kV) kann die Empfindlichkeit der Kamera eingestellt werden.

tralen Empfindlichkeit der CCD-Kamrea liegt, so kann ein Bildverstärker als Wellenlängenkonverter genutzt werden. Die einfallenden, kurzwelligen Photonen werden in langwelligere transformiert, wobei deren spektrale Position vom verwendeten Phosphor bestimmt wird. Dadurch kann eine optimale Anpassung der spektralen 
Empfindlichkeit der nachgeschalteten CCD-Kamera erreicht werden. Eine weitere Aufgabe des Bildverstärkers ist in der Aufnahme von sehr schwachen Signalen zu sehen. Die Vervielfachung der Primärelektronen innerhalb des MCPs führt zu einer effektiven Verstärkung, die eine Leuchterscheinung auf dem Phosphorschirm auslöst. Diese hat eine so starke Leuchtkraft, dass sich das auf den direkt angekoppelten CCD-Sensor aufgenommene Signal deutlich vom Hintergrundrauschen abhebt.

Ein weiterer Vorteil des Bildverstärkers ist, dass dieser sehr kurze Belichtungszeiten ermöglicht im Gegensatz zum CCD-Sensor. Letzterer benötigt eine minimale Belichtungszeit von ungefähr $1 \mu \mathrm{s}$. Sehr kurze Ereignisse wie zum Beispiel Fluoreszenzabklingzeiten, bei denen ein zeitlich konstantes und hell leuchtendes Hintergrundsignal vorliegt, sind so nicht erfassbar. Nur mit Hilfe der kurzen Belichtungszeiten des Bildverstärkers, die sich im ns-Zeitbereich befinden, können solche Phänomene detektiert werden.

Es wird ersichtlich, dass das Zeitfenster des Bildverstärkers die eigentliche Messzeit der CCD-Kamera darstellt. Um keine Verzögerung der Reaktionszeit des CCDSensors zu erhalten, liegt das Zeitfenster des Bildverstärkers innerhalb des Zeitfensters des CCD-Sensors. Dies ermöglicht neben der Einzelaufnahme auch eine on-chipIntegration, bei der ein sehr langes Zeitfenster des CCD-Sensors eine Vielzahl von Verstärker-Zeitfenstern beinhaltet. Dabei findet eine Aufsummierung eines schwachen Meßsignals statt, was eine Erhöhung des $S / N$-Signals hervorruft. Abbildung 5.8 zeigt den zeitlichen Verlauf der unterschiedlichen Zeitfenster.

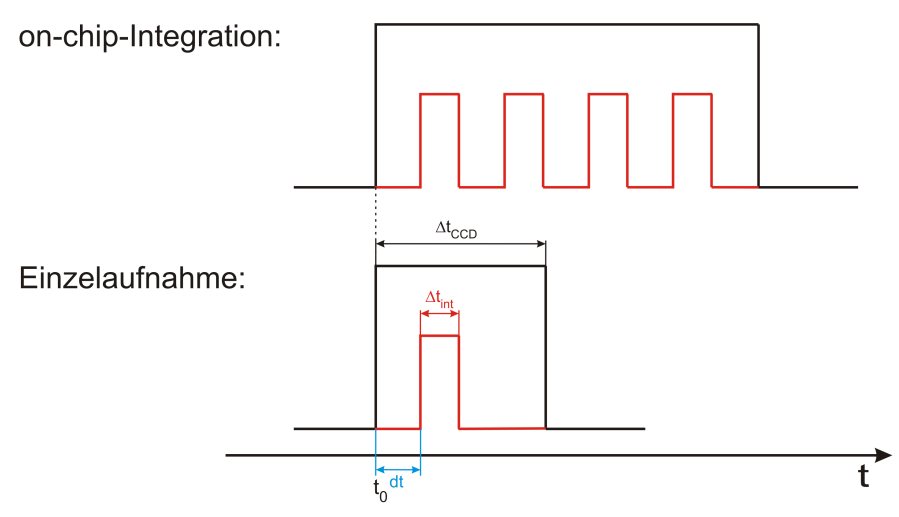

Abbildung 5.8: Zeitlicher Verlauf der Zeitfenster. Der CCD-Sensor wird bei $t_{0}$ aktiviert (schwarz) und bleibt bis zur Zeit $\Delta t_{C C D}$ offen. Der Bildverstärker (rot) ist mit einer Verzögerung von $d t$ aktiv und liegt innerhalb des Zeitfensters des CCD-Sensors. Die eigentliche Messzeit wird durch $\Delta t_{i n t}$ bestimmt. Neben der Einzelaufnahme kann auch eine on-chip-Integration durchgeführt werden. Dabei liegen mehrere Verstärker-Zeitfenster innerhalb des Sensorfensters. 


\subsection{Spektrometer}

Das von dem Faserbündel detektierte Fluoreszenzsignal wurde mit Hilfe eines Spektrometers erfasst und spektral aufgelöst. Der prinzipielle Aufbau des Gerätes ist in Abbildung 5.9 ersichtlich. Es handelt sich dabei um einen Czerny-Turner-Monochromator, bei dem das durch den Eintrittsspalt eintreffende Licht mit Hilfe eines Gitters spektral zerlegt und auf der Austrittsspaltebene wieder abgebildet wird. Die spektrale Isolierung erfolgt durch den Austrittsspalt selbst. Mit Hilfe eines sphärischen Spiegels (Spiegel 1) wird das Licht kollimiert und auf ein Gitter gelenkt, an dem die spektrale Auflösung stattfindet. Ein zweiter sphärische Spiegel (Spiegel 2) fokussiert das spektral aufgelöste Licht auf die Spaltebene des Austritts. Um das gesamte

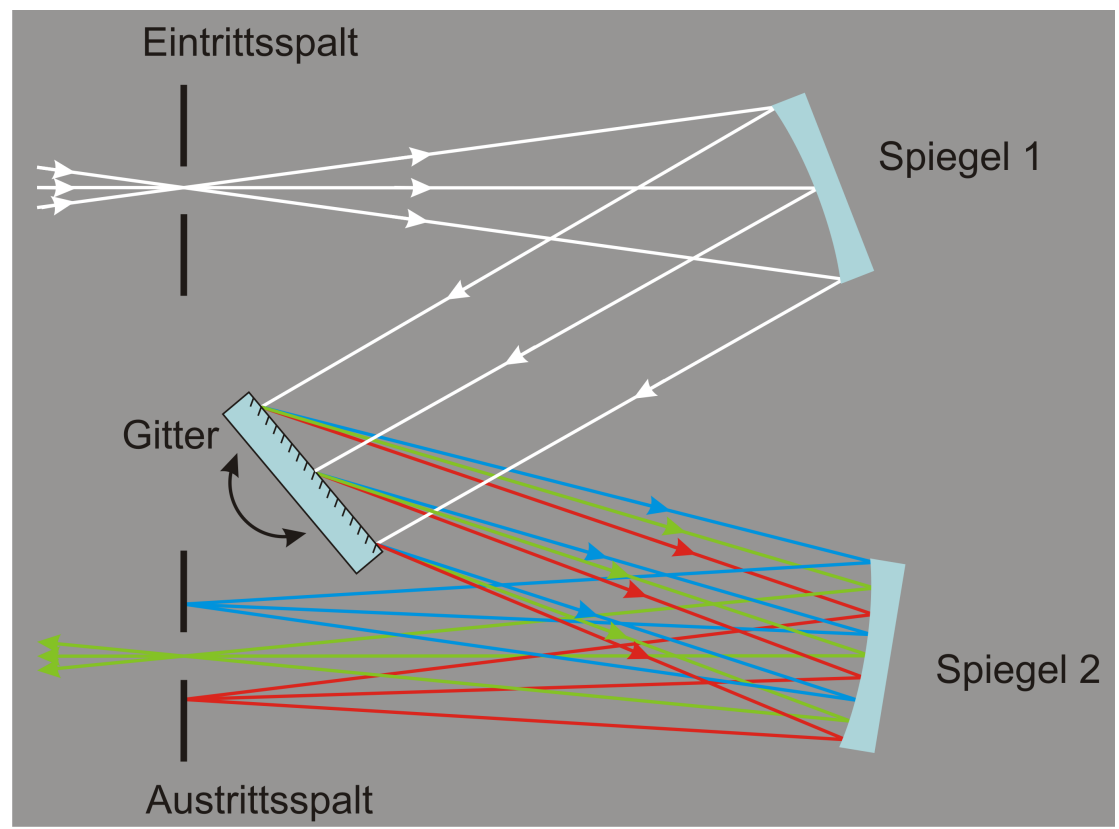

Abbildung 5.9: Das im Eintrittsspalt fokussierte Licht wird mit Hilfe eines sphärischen Spiegels kollimiert und auf ein drehbares Gitter gelenkt. Dort wird durch Beugung das Licht spektral aufgelöst und mit einem zweiten Spiegel in der Ebene des Austrittsspalts fokussiert. Die durch den Spalt durchgelassene Wellenlänge wird durch den Drehwinkel des Gitters eingestellt.

Licht zur spektralen Analyse zur Verfügung zu haben, muss dieses einen Teil oder die Gesamtfläche des Gitters ausleuchten. Ein Überstrahlen würde zu Lichtverlusten führen. Um diesen Verlust zu minimieren, findet eine Anpassung der detektierten Lichtquelle an das Spektrometer durch die sogenannte F-Zahl statt. Diese auch als $f$ /\# bezeichnete Größe tritt ebenfalls in der Fotografie auf und wird als Blendenzahl bezeichnet. Sie ist dort definiert als der Quotient aus der Brennweite $f$ der 
Objektivlinse und dem Durchmesser $D$ der Eintrittspupille.

$$
f / \#=\frac{f}{D}
$$

Streng genommen gilt dies nur für paraxiale Strahlen. Unter Verwendung der in Abbildung 5.10 definierten Größen und bei Annahme einer Luftumgebung mit dem Brechungsindex $\mathrm{n}=1$ ergibt sich

$$
f / \#=\frac{f}{D}=\frac{1}{2 \cdot n \cdot \sin \Theta}
$$

Gemäß Gleichung 5.5 ensteht eine Verknüpfung zwischen der F-Zahl und der numerischen Apertur NA

$$
f / \#=\frac{1}{2 \cdot N A}
$$

Mit Hilfe von Gleichung 5.18 kann eine Anpassung durchgeführt werden. Das in

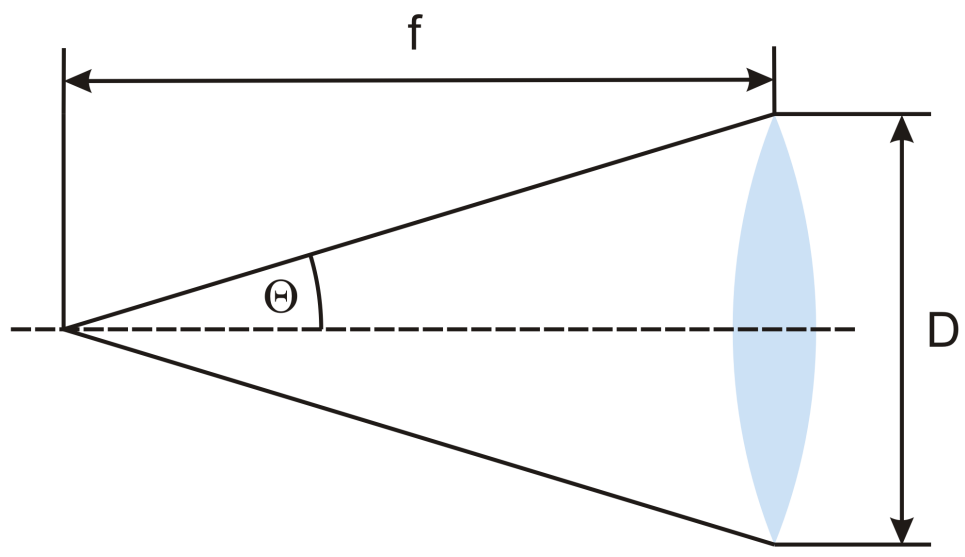

Abbildung 5.10: Verknüpfung zwischen F-Zahl und numerischer Apertur. Die

F-Zahl beschreibt die Fähigkeit einer Linse, Licht bis zu einem bestimmten

Winkel $\Theta$ zu sammeln.

dieser Arbeit verwendete Spektrometer der Firma Acton Research vom Typ SpectraPro $275 i$ hat eine Spiegelbrennweite von $f=275 \mathrm{~mm}$ und eine $f / \#$ von 3,8 und somit eine NA von 0,13. Das bedeutet, dass das Gitter bei diesem $f / \#$-Wert gerade genau ausgeleuchtet wird. Innerhalb dieser Arbeit wird die Einkopplung mit Hilfe von Fasern durchgeführt (siehe Abbildung 5.11). Idealerweise sollten die Fasern eine NA von 0,13 haben. Quarzglasfasern sind zwar mit solchen NA-Werten erhältlich, allerdings wird durch das Verschmelzen der Faserenden die NA wieder erhöht, aufgrund des Einsatzes einer abschließenden Glasplatte. Daher hat das eingesetzte Faserbündel eine numerische Apertur von $N A=0,37 \mathrm{bzw}$. eine $f / \#$ von 1,35. Diese starke Differenz der F-Zahl könnte mit einem Zwei-Linsen-System theoretisch korrigiert werden. Die dabei entstehenden langen Brennweiten würden allerdings weitere 


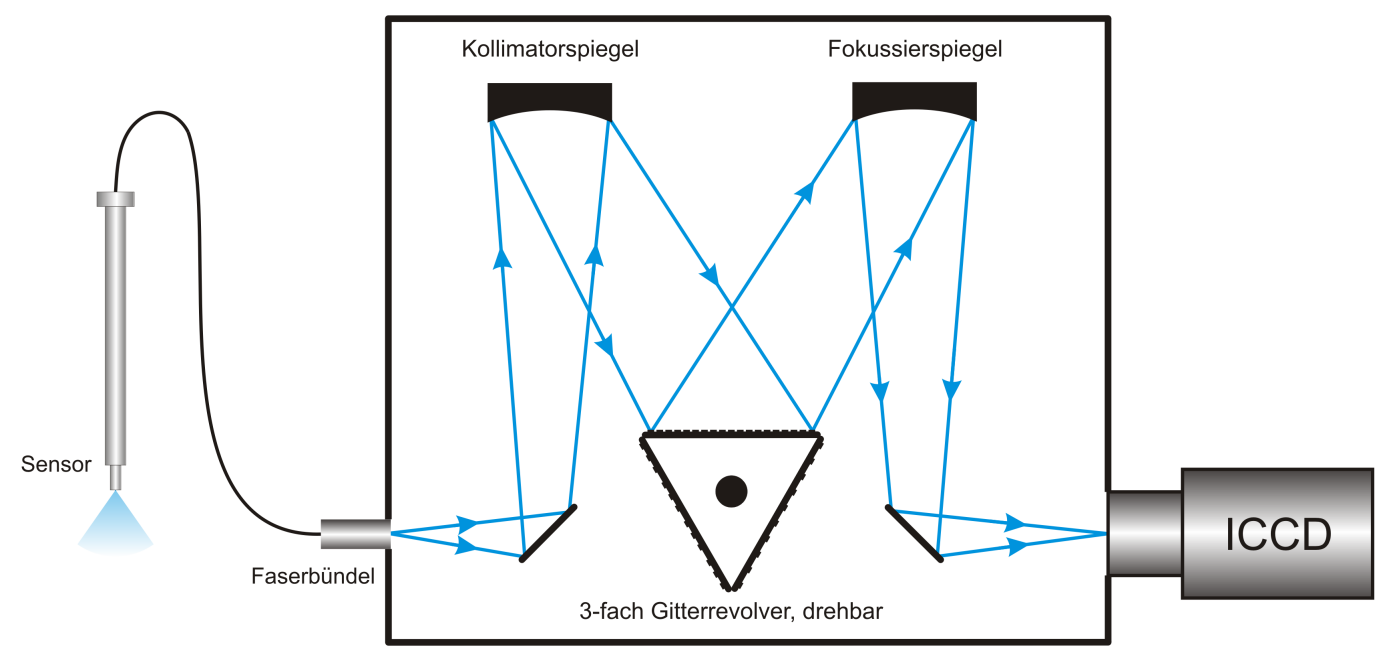

Abbildung 5.11: Schematischer Aufbau des Detektorsystems. In der Ebene des Eintrittsspalts ist das Faserbündel platziert. Die Positionierung des CCDSensors in der Austrittsspaltebene läßt die simultane Detektion mehrerer Wellenlängen zu. Das Spektrometer kann mit drei verschiedenen Gittern ausgestattet werden, welche durch den Gitterrevolver schnell auswechselbar sind.

Verluste nach sich ziehen. Diverse Linsenaufbauten zeigten im Experiment keine Verbesserung der Signalstärke. Daher wurde der Signalverlust aufgrund der deutlichen Überstrahlung des Gitters akzeptiert, da das detektierte Signal ausreicht, um die Realisierbarkeit des physikalischen Verfahrens zu überprüfen. Die Verwendung von Low-NA-Fasern, oder eines Spektrometers mit entsprechender $f$ /\# wäre der sinnvollste Schritt zur Optimierung des Detektorsystems. Sofern eine Möglichkeit besteht, sollte dies in weiterführenden Experimenten miteinbezogen werden.

\subsection{Spektrale Filter}

Mit Hilfe von spektralen Filtern wird eine Separation des Meßsignals von anderen Signalen erreicht. So ist es notwendig, das zu detektierende Fluoreszenzsignal von störenden Größen wie zum Beispiel dem vom Laser erzeugten Steulicht zu trennen. Um das emittierte Fluoreszenzlicht selektieren zu können, wurden verschiedene Langpaßfilter der Firma Schott anhand der Datenblätter ([45]) vorselektiert und der am besten geeignete Filter aufgrund von Transmissionsmessungen am Spektrometer Perkin Elmer Spectrometer Lambda 19 bestimmt. 


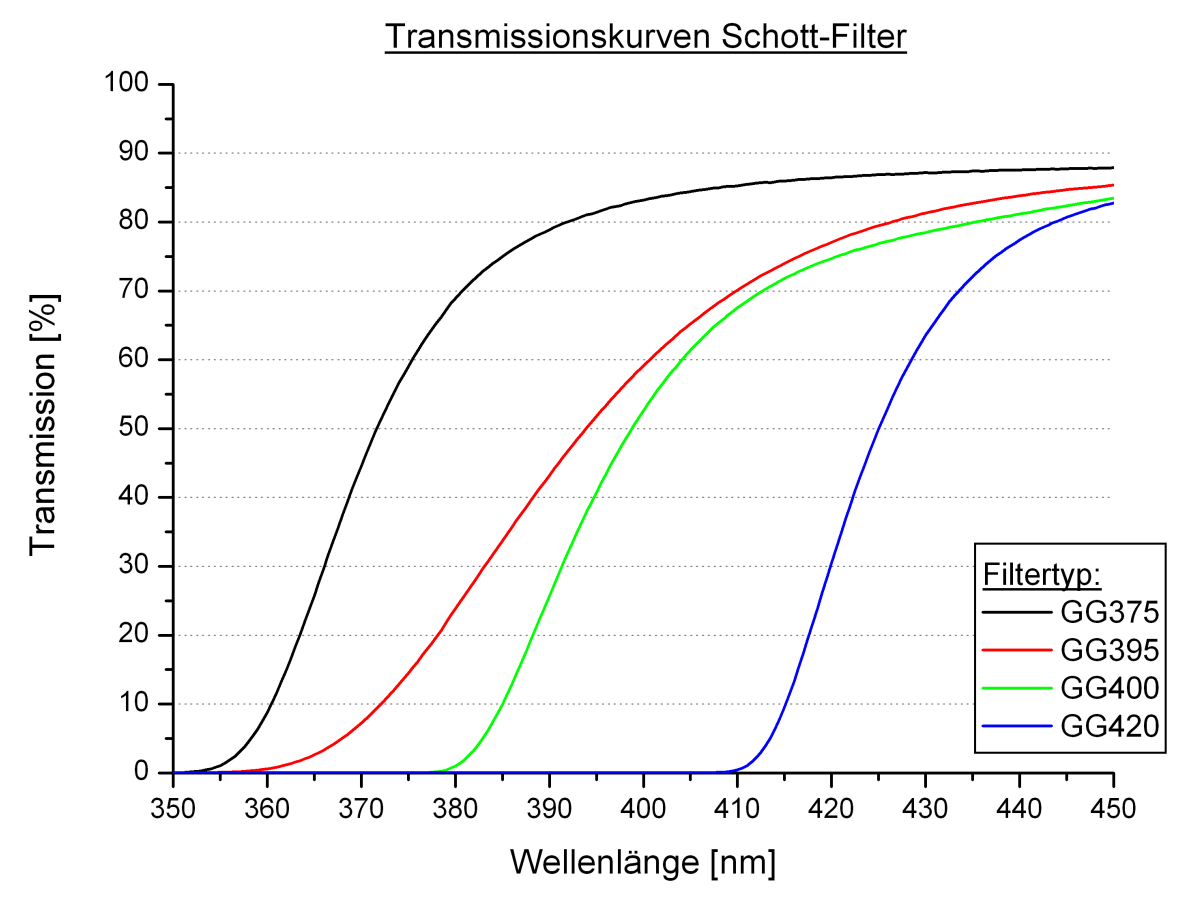

Abbildung 5.12: Gemessene Transmissionskurven diverser Langpaßfilter zur Separation des Fluoreszenzlichts. Für die vorgestellten Messungen wurde der Filter GG375 verwendet.

Die gemessenen Transmissionskurven in Abbildung 5.12 zeigen, dass der Filtertyp GG375 eine hohe Transmission für den spektral relevanten Bereich von 400-420nm besitzt, bei nahezu vollständiger Abschirmung der Laserwellenlänge von $355 \mathrm{~nm}$. Zwar ist eine vollständige Unterdrückung bei dem Filtertyp GG395 gegeben, die deutlich besseren Transmissionswerte von GG375 liefern jedoch mehr Fluoreszenzintensität. Somit wurde der Filter GG375 in der Detektionseinheit installiert. Dieser befindet sich innerhalb des Spektrometers zwischen der Austrittsfläche des Faserbündels und dem ersten Umlenkspiegel (siehe Abbildung 5.11).

\subsection{Optische Messverfahren an Motoren}

Um physikalische und chemische Vorgänge der technischen Verbrennung untersuchen zu können, muss eine Möglichkeit geschaffen werden, optische Zugänge zum Brennraum zu erhalten. Aufgrund der konstruktiven Komplexität eines Motors ist dies eine besondere Herausforderung, die auf unterschiedliche Wege gelöst wurde. Bei einem Verbrennungsmotor handelt es sich in den meisten Fällen um ein Mehrzylindersystem mit identischen Anordnungen der einzelnen Bauteile. Im Wesentlichen 
besteht jeder Zylinder aus einem Kolben, dem Zylinderblock, dem Zylinderkopf und einem Kurbelgehäuse. Dadurch gibt es drei Möglichkeiten für einen optischen Zugang. Ein Zugang oberhalb des Brennraums über den Zylinderkopf, einen seitlich des Brennraums über die Zylinderwand und unterhalb des Brennraums durch den Kolben.

Für eine direkte Beobachtung der Verbrennung reicht ein Zugang. Für die meisten optischen Verfahren werden jedoch zwei oder mehr Zugänge benötigt. Dies ist zum Beispiel der Fall, wenn eine Lichtquelle zur Anregung benutzt wird und man die angeregte Substanz beobachten möchte. Je nach Bauart kann der Zugang nur eine örtlich begrenzte Beobachtung ermöglichen. Weiterhin besteht eine Begrenzung der Beobachtungsdauer durch ansteigender Verschmutzung aufgrund von Verbrennungsrückständen. Ein weiterer Effekt ist, dass optische Motoren meistens eine niedrigere Kompressionsrate als Serienmotoren besitzen. Um den motorischen Bedingungen standhalten zu können, gibt es nur bestimmte Materialien, welche für die optischen Fenster verwendet werden können. Für einen Wellenlängenbereich von 350 bis 2000nm ist die Glasart BK7 geeignet. Bei Untersuchungen im UV-Bereich ist es allerdings notwendig, Quarzglas zu verwenden. Das beste Material hingegen ist Saphir. Dieser aus Aluminiumoxid bestehende Kristall hat für die Anwendung hervorragende mechanische und optische Eigenschaften und bietet aufgrund der Transmission von 180 bis 5500nm eine breite spektrale Anwendung. Nachteil sind die hohen Anschaffungskosten.

Für den optischen Zugang im Zylinderkopf stellt sich die Schwierigkeit, dass in den meisten Motoren die Ansaug- und Auslasskanäle mit den entsprechenden Ventilen und zum Teil auch die Zündkerze und Einspritzung im Zylinderkopf integriert sind. Somit muss eine Modifikation des Versuchsmotors durchgeführt werden. Es wurden im Laufe der Jahre viele verschiedene Versuchsmotoren entwickelt, um diverse Phänomene wie zum Beispiel die Selbstzündung und das Klopfen zu untersuchen ([46]). Von Felton et al. [47] und Ghandhi et al. [48] wurde ein Motor verwendet, welcher sowohl mit einer äußeren Gemischbildung als auch mit Direkteinspritzung arbeitet. Auch Dieselmotoren wurden 1969 erstmals von Scott [49] zur Untersuchung herangezogen. Bei großen Dieselmotoren mit zwei Auslasskanälen wurde von Dec et al. [50] ein Auslasskanal als optischer Zugang umfunktioniert und mit Hilfe einer Mehrloch-Einspritzdüse das Spray des Kraftstoffes so umgelenkt, dass dies direkt zwischen Fenster und Kolben positioniert war. Die Einlassventile wurden nicht verändert und somit konnte die Strömungsentwicklung eines Serienmotors reproduziert werden. Aufgrund dieser Konfiguration gelang es, die Sprayausbildung und deren Verbrennung unter nahezu realen Betriebszuständen zu analysieren. Weiterhin wurde von Zhao [51] ein Einzylinder-Dieselmotor entwickelt, der im Zylinderkopf eine separate Wirbelkammer hatte, die mit dem Brennraum verbunden war und drei optische Zugänge besitzt.

Durch die oberhalb des Brennraums befindlichen Ventile und Kanäle in modernen Motoren wurde ein optimaler Zugang geschaffen, um die technische Verbrennung zu 
beobachten. Hierbei wird ein Fenster in den Kolbenboden eingelassen und mit Hilfe eines $45^{\circ}$-Spiegels, welcher sich direkt unterhalb des Fensters befindet, ein Einblick in den Brennraum generiert. Bowditch [52] realisierte 1961 als erster dieses Verfahren bei General Motors. Der Vorteil ist, dass keine Modifikationen des Zylinderkopfes erbracht werden müssen und so die Strömungseigenschaften völlig störungsfrei untersucht werden können. Zusätzlich kann hier ein großer optischer Zugang erzeugt und damit eine großflächige Untersuchung vorgenommen werden. Allerdings ist diese Untersuchungsmethode anfälliger auf Fensterverschmutzungen in Bezug auf Öl und Ruß, so dass die Beobachtungsdauer auf ein paar Zyklen beschränkt ist.

Mit der Anwendung der ortsaufösenden Laserdiagnostik wurden zusätzliche Fenster zwischen dem Zylinderkopf und dem Zylinderblock notwendig. Alternativ zu einzeln angebrachten Fenstern kann ein Glasring eingesetzt werden, wobei ein $360^{\circ}$-Einblick entsteht, der besonders für PIV-Messungen geeignet ist. Bei letzterem ist darauf zu achten, dass der Kontakt des Kolbens mit dem Ring keine Zerstörung hervorruft. Vor allem ist der Kontakt mit den harten Kolbenringen zu vermeiden. Zwischen Kolben und der Wandung ist ein leichter Ölfilm, welcher den Verschleiß hinauszögern soll. Dieser Ölfilm ist wiederum ein störender Faktor, wenn der Kolben über einen Glasring gleitet. Um diese beiden Probleme zu verhindern, kommen Kolbenringe aus speziellem Kunststoff (Polyamidimid, auch als Torlon ${ }^{\circledR}$ bekannt) zum Einsatz. Bei dieser Methode kann der Glasring fast beliebig verlängert werden, sofern ein größerer Bereich des Brennraums untersucht werden soll. Detaillierte Informationen über dieses Verfahren sind bei Bates [53] enthalten. Ein Nachteil ist, dass die thermodynamischen Eigenschaften des Motors bei der Verwendung eines Glasrings am stärksten von realen Motoren abweichen. In Abbildung 5.13 ist ein Glasringmotor schematisch dargestellt. Genauere Beschreibungen der hier erwähnten Verfahren sind in [51] zu finden.

Der Fortschritt der minimal-invasiven Technologie in den letzten Jahren ermöglicht es, die Zündkerzenöffnung immer mehr für die Motordiagnostik zu erschließen. Dieses Verfahren benötigt so gut wie keine konstruktive Veränderung des Motors, dessen thermodynamischen Eigenschaften somit erhalten bleiben. Besonders der Zündort kann örtlich exakt untersucht werden. Auch für die hier vorgestellte Arbeit wurde dieser Weg in den Brennraum gewählt und die Entwicklung eines Sensorsystems unter diesen Bedingungen vorangetrieben.

\subsection{Entwicklung eines Sensorsystems für motorische Messungen}

Neben Untersuchungen an einer druck- und temperaturregelbaren Messzelle wurde ein Sensorsytem entwickelt, welches Fluoreszenzmessungen innerhalb eines Verbrennungsmotors ermöglicht. Hierbei wurde die Zündkerzenöffnung als Zugang zum 


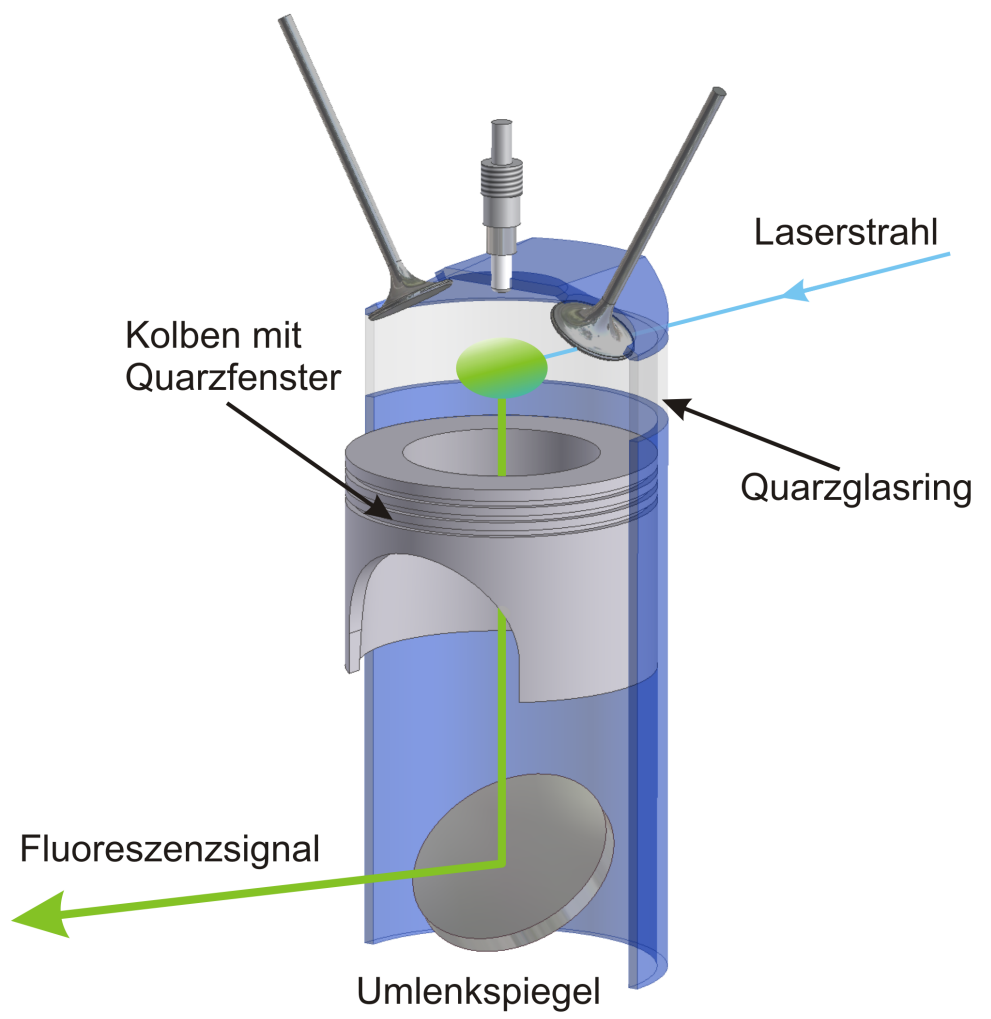

Abbildung 5.13: Prinzipieller Aufbau eines Glasringmotors Der Kolben kann wie hier gezeigt eine Aussparung haben, um den optischen Weg nicht zu beeinträchtigen. Für Messungen zur Visualisierung, wie z.B. PIV, genügt der Glasring bei orthogonaler Anordnung der Lichtquelle und der Kamera.

Brennraum gewählt. Dies hat den Vorteil, dass keine konstruktiven Veränderungen eines Serienmotors durchgeführt werden müssen und der Ort der Initialzündung recht genau untersucht werden kann. Ein Problem stellt jedoch die Entwicklung eines zündfähigen Sensors dar. Der entstehende Zündfunke darf sich nicht zu nah an den optischen Komponenten befinden, da diese sonst beschädigt werden können. Dies ist aber aufgrund der minimalen Abmaße recht schwer zu erfüllen. Weiterhin ist ein großes Problem das sehr starke Signal der Plasmazündung, welches das eigentliche Messsignal überstrahlt. Im Hinblick auf die Untersuchung des Gemischzustandes vor der Verbrennung ist hier ein zündfähiger Sensor nicht notwendig. Die diesen Zustand beeinflussenden physikalischen Parameter beschränken sich auf die Strömungscharakteristik, die Lage des Zündortes und die Kompressionsfähigkeit des untersuchten Motorsystems.

Basierend auf diesen Erkenntnissen wurde ein Sensor mit der Firma LaVision entwickelt und die ersten Untersuchungen mit Hilfe von Messungen an der temperaturkontrollierten Druckzelle durchgeführt, um die Einsatzfähigkeit des Systems zu 
prüfen. Die dabei gemachten Erfahrungen wurden mit in die Entwicklung eines zweiten Sensorsystems einbezogen.

\subsubsection{Sensor 1. Generation}

Der erste Sensor wurde in Zusammenarbeit mit LaVision entwickelt und konstruiert. Da wie schon erwähnt der Zustand vor der Verbrennung erfasst werden soll, konnte die Konstruktion des Sensors dahingehend vereinfacht werden, dass keine Zündelektronik integriert werden musste. Somit sind Messungen im geschleppten, ungezündeten Motorbetrieb realisierbar. Bei diesem Sensorkonzept wird das Anregungslicht des Lasers über einen Quarzstab mit einem Durchmesser von $d \leq 3 \mathrm{~mm}$ in den Brennraum geführt, welcher axial im Sensor angeordnet ist und in den Detektionsraum hineinragt. Dieser Quarzstab besitzt auskoppelseitig einen $45^{\circ}$-Anschliff, so dass eine orthogonale Ausstrahlung auf die verspiegelte Innenwand des Sensorkopfes entsteht. An der direkt angestrahlten Fläche befindet sich eine angewinkelte Spiegelfläche, die das Licht auf die anderen Bereiche der Innenwand reflektiert, wodurch aufgrund von Mehrfachreflexionen eine vollständige Ausleuchtung des Detektionsraumes erreicht wird. Somit wird Gas, welches sich innerhalb dieses Volumens befindet, zur Fluoreszenz angeregt. Die Detektion des Fluoreszenzlichtes erfolgt über Quarzglasfasern, die ringförmig um die Quarzstabführung angeordnet sind. Die Fasern führen das Fluoreszenzlicht zum Spektrometer zur weiteren Analyse. Abbildung 5.14 verdeutlicht noch einmal das Prinzip dieses Sensors.

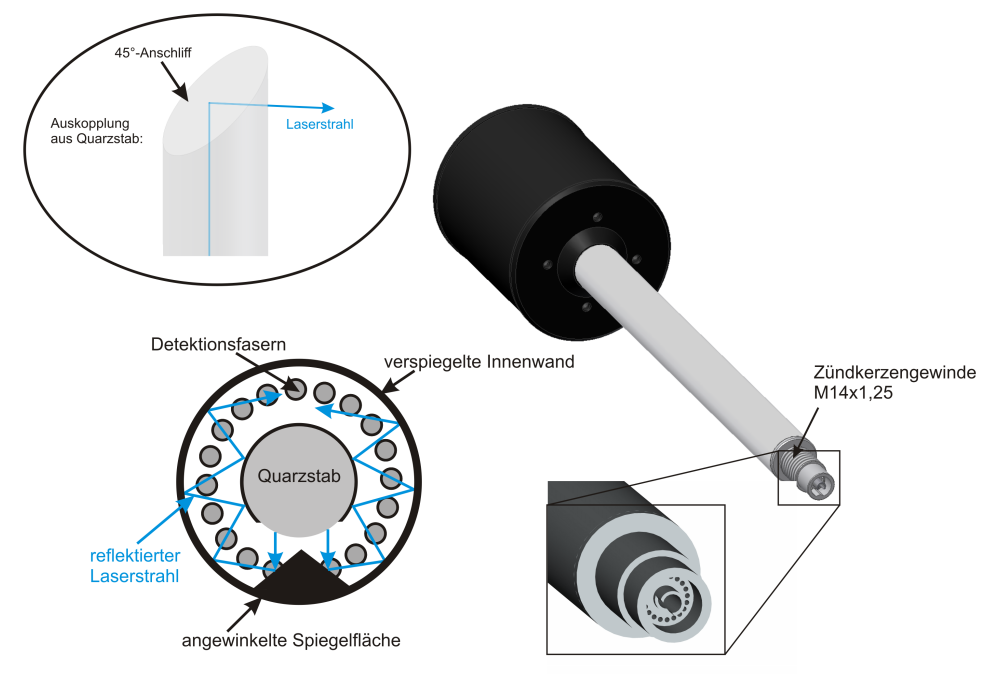

Abbildung 5.14: Aufbau des ersten Sensorkonzepts. Mit Hilfe des 45-Anschliffs wird das Laserlicht aus dem axial angeordneten Quarzstab ausgekoppelt (oben links). Durch die angewinkelte Fläche soll eine Mehrfachreflexion des Strahls zur Fluoreszenz anregen. 
Die Detektionsfasern haben einen Durchmesser von $d=600 \mu \mathrm{m}$ und haben einen Schutzmantel aus Polyamid. Jeweils fünf der insgesamt zwanzig ringförmig angeordneten Detektionsfasern werden in einem Steckersystem der Firma Spinner (Details siehe Anhang A.1) integriert und mit den zum Spektrometer weiterführenden Fasern verbunden.

Die mit diesem Sensorkonzept durchgeführten Untersuchungen dienten zur Überprüfung der Einsatzfähigkeit unter motorischen Bedingungen, welche mit Hilfe einer temperierbaren Druckzelle generiert wurden. Da hier keine motorbedingten Schwingungen auftreten, wurde die Einkopplung des Laserstrahls in Form eines Freistrahls gewählt. Das Sensorsystem ist jedoch so konzipiert, dass eine Einkopplung auch über eine Quarzglas-Faser mit einem Durchmesser von $d=1000 \mu \mathrm{m}$ über ein StandardSMA-Steckersystem möglich ist. Die laserseitige Stirnfläche der Faser ist dabei mit einer sogenannten High-Power-SMA-Kupplung LaserConnect 100 versehen worden. Diese hat ein freistehendes Faserende ohne Ummantelung. Das hat den Vorteil, dass am Faserrand auftreffende Laserstrahlung kein Mantelmaterial verdampfen kann, welches sich auf die Stirnfläche niederschlägt und dadurch die Faser blind werden läßt.

Obwohl die in Kapitel 6 vorgestellten Resultate recht vielversprechend sind, gaben die Messungen Hinweise darauf, dass dieses Konzept einige Schwachstellen bezüglich einer längerfristigen Einsatzdauer aufweist. Ein Problem war die starke Abnahme des Meßsignals innerhalb fortlaufender Messreihen gegenüber den ersten Messreihen. Bei genauerer Betrachtung des Detektionsvolumens im Sensorkopf war eine Zerstörung der angewinkelten Spiegelfläche zu erkennen. Dies kam dadurch zustande, dass die auskoppelnde Mantelfläche des Quarzstabs mit einem Durchmesser von $3 \mathrm{~mm}$ wie eine Zylinderlinse wirkte und durch den geringen Radius von 1,5mm eine Fokussierung des Laserstrahls auf der Oberfläche verursachte. Die noch recht hohe Laserenergie ließ dort ein Plasma enstehen, welches zur Zerstörung führte. Ein weiterer Aspekt ist, dass ein absolut orthogonaler Austritt des Laserlichtes gewährleistet werden muss, damit der reflektierte Strahl innerhalb des Messvolumens bleibt und sich kreisförmig um die Quarzstabführung ausbreitet. Eine kleine Abweichung dieser Orthogonalität würde das Anregungslicht aus dem Sensorkopf hinaus reflektieren. Aber gerade diese Bedingung erwies sich als schwer zu realisieren, was daran ersichtlich wurde, dass die Fasern in der Nähe der auskoppelnden Quarzstabmantelfläche den größten Anteil zum Gesamtsignal beitrugen, während schon die benachbarten Fasern kaum oder gar kein Signal mehr lieferten. Eine Änderung durch Planschliff der Mantelfläche hatte keine Verbesserung zur Folge. Aufgrund dieser Erfahrungen wurde ein verbessertes Konzept entwickelt, welches im Sensorsystem der 2. Generation implementiert wurde. 


\subsubsection{Sensor 2. Generation}

Das zweite Konzept des Sensors wurde in Zusammenarbeit mit der Firma Ceram Optec konstruiert. Hierbei wurden die Erfahrungen des vorherigen Sensors mit den konstruktiven Möglichkeiten von CeramOptec vereint. Eine Zielsetzung bei der Entwicklung war eine sehr nahe Positionierung der Detektionsfasern an die Anregungsstelle, um Absorptionsverluste gering zu halten. Somit musste ein geringer Abstand zwischen der Anregungs- und Detektionsfaser realisiert werden. Aufgrund der technischen Möglichkeit einer definierten Platzierung der einzelnen Fasern und dem Verschmelzen der Faserbündel, konnte diese Zielsetzung erfüllt werden.

Dadurch besteht das Sensorsytem im Wesentlichen aus zwei Faserbündeln. Jedes Bündel beinhaltet 140 Quarzglas-Fasern mit einem Kerndurchmesser von $189 \mu \mathrm{m}$ mit einer NA von 0,26. Die jeweiligen Endflächen bestehen aus einer Quarzglasplatte, die mit den einzelnen Fasern miteinander verschmolzen sind. Aufgrund dieser verschmolzenen Endfläche ändert sich die NA des Bündels auf den Wert 0,37. Das zur Anregung verwendete Faserbündel besitzt eine Eintrittsferrule aus Edelstahl mit einem effektiven Durchmesser von 2,4 mm und ist im Sensorkopf zusammen mit dem Detektions-Faserbündel verschmolzen. Dabei wurde eine definierte Anordnung der Anregungs- und Detektionsfasern insofern generiert, dass diese jeweils abwechselnd eine Reihe bilden (siehe auch Abbildung 5.15).

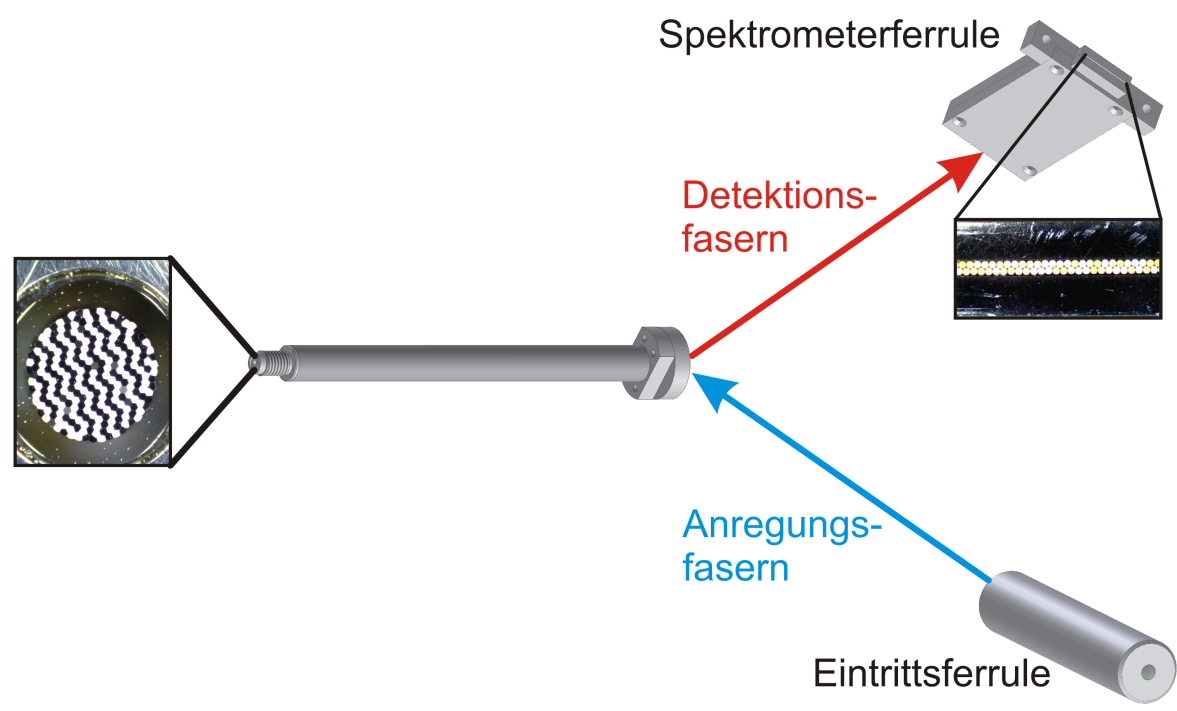

Abbildung 5.15: Struktur des Sensorsystems. In der linken Vergrößerung ist die Anordnung der Anregungs- (hell) und Detektionsfasern (dunkel) zu erkennen. Die Spektrometerferrule ist auf den Spalt des verwendeten Spektrometers angepasst. Dadurch entstanden zwei Reihen mit je 70 Fasern (Vergrößerung rechts). 
So ist gewährleistet, dass Anregungs- und Detektionsfasern nebeneinander positioniert sind. Die so gestaltete Sensorferrule besteht somit aus 280 verschmolzenen Fasern und hat einen effektiven Durchmesser von 3,4 mm. Die Endfläche des Faserbündels zur Detektion besitzt eine Spektrometerferrule, die an das Spektrometer SpectaPro $275 i$ angepasst ist. Innerhalb dieser Ferrule sind die 140 Detektionsfasern in zwei Reihen in engster Packung angeordnet, wie in Abbildung $5.15 \mathrm{zu}$ sehen ist, wobei eine Anpassung auf die Spaltmaße des Eintrittsspalts durchgeführt wurde. Die von den Fasern ausgefüllte Fläche beträgt 0,4x14 mm. Aufgrund der Möglichkeit der Faserverschmelzung sind Sensor und Bündel fest miteinander verbunden, um Verluste durch Steckverbindungen zu vermeiden. In Anhang A.1 und A.2 befinden sich detaillierte Konstruktionszeichnungen der entwickelten Sensorsysteme. Abbildung 5.16 zeigt schematisch die Lage des Sensors im Motor.

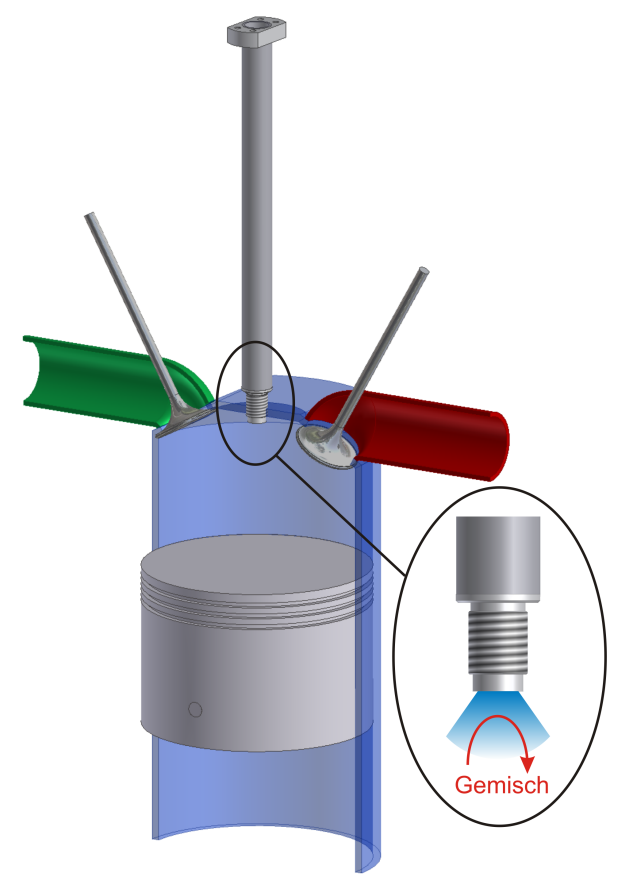

Abbildung 5.16: Einsatz der Sensors der 2. Generation im Motor (schematisch). Unterhalb des Sensors wird das vorbeiströmende Gemisch mit Hilfe der aus den Fasern austretenden Laserstrahlung zur Fluoreszenz angeregt. Die benachbarten Detektionsfasern leiten das Fluoreszenzlicht zum Spektrometer. 


\section{Kapitel 6}

\section{Experimentelle Untersuchungen des angewandten Messverfahrens}

Im folgenden Kapitel werden die experimentellen Arbeiten vorgestellt, die für die Entwicklung des Temperatur-Messverfahrens durchgeführt wurden. Die Fragestellung der ersten Untersuchungen bezog sich dabei auf die spektroskopischen Eigenschaften des Formaldehyds und dessen Eignung für das Verfahren. Dem schloss sich eine Überprüfung der verwendeten Baugruppen an, um eine Abschätzung des Einflusses auf das Messergebnis zu bekommen. Das Kernstück der Experimente stellen jedoch die Untersuchungen an der Druckkammer dar. Diese Versuche dienten als Machbarkeitsstudie für die Anwendung in der Motordiagnostik und der Entwicklung eines applikationsangepassten Sensorsystems. Den Abschluss bilden Messungen am Motor.

\subsection{Experimenteller Aufbau}

Ein großer Teil der Messungen wurde an einer druckfesten, heizbaren Edelstahlkammer durchgeführt. Die Kammer hat eine Grundfläche von 130x130mm² und eine Höhe von $180 \mathrm{~mm}$. Über drei Quarzglasfenstern ist sie großräumig optisch zugänglich. Die vierte Kammerseite ist an einem Halterungsflansch befestigt, in dem Verschraubungen für Leitungen von Gaszufuhr und Gasauslaß, sowie für ein Manometer zur Druckaufnahme integriert sind. Weiterhin beinhaltet der Halterungsflansch zwei druckdichte Thermoelement-Durchführungen zur Bestimmung der Kammerinnentemperatur. Die Thermoelemente wurden so plaziert, das sich der Messort nahe des Laserstrahls bei den Freistrahlmessungen und nahe des Sensorkopfes bei den Sensormessungen befand. Die Temperaturwerte wurden mit einem digitalen 4-KanalThermometer (VOLTCRAFT 304) erfasst. Die Kammer besitzt acht Längsbohrungen, von denen vier der Wasserkühlung dienen. In den anderen vier Bohrungen be-

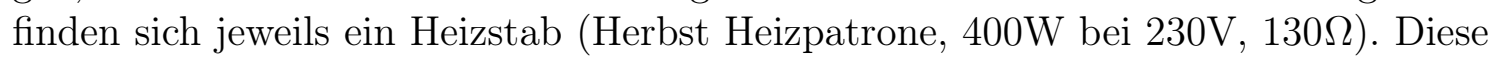
können eine maximale Temperatur von $200^{\circ} \mathrm{C}$ erzeugen. Mit Hilfes eines Trenntrafos kann die Temperatur geregelt werden. Die über Flansche montierten Quarzglasfenster haben eine Abmessung von BxHxT 66x116x31,8mm und halten einem Druck 
von bis zu 30bar stand (Abbildung 6.1). Das Fenstermaterial ist Suprasil III, welches eine hohe Festigkeit gegenüber den eingestrahlten Laserintensitäten hat und ebenfalls eine gute UV-Transmission aufweist ( $\mathrm{T}>90 \%$ für $\lambda>200 \mathrm{~nm}$ ). Über eine 50mm Bohrung können die Sensorsysteme mit Hilfe eines selbstkonstruierten Flansches in die Kammer hineinragen.

\section{Draufsicht}

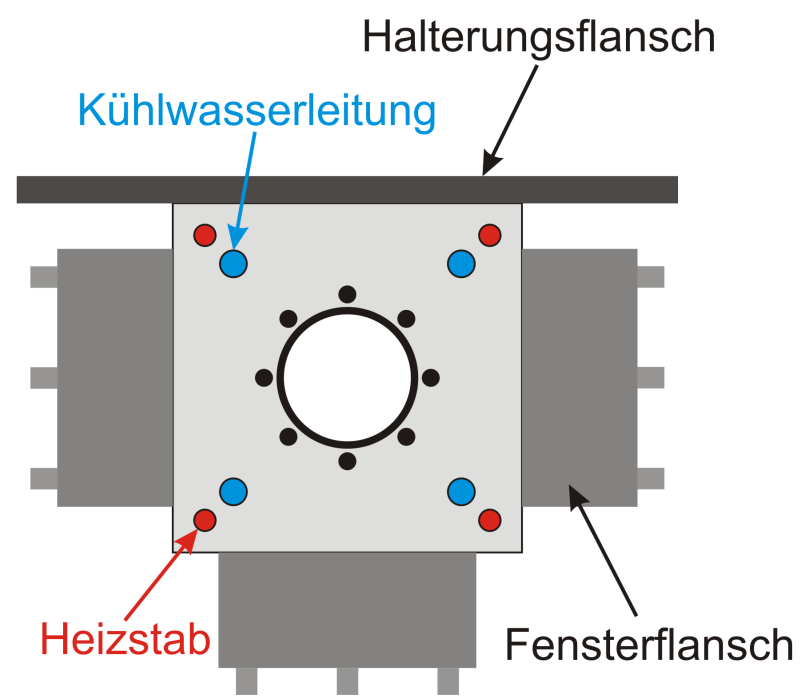

\section{Seitenansicht}

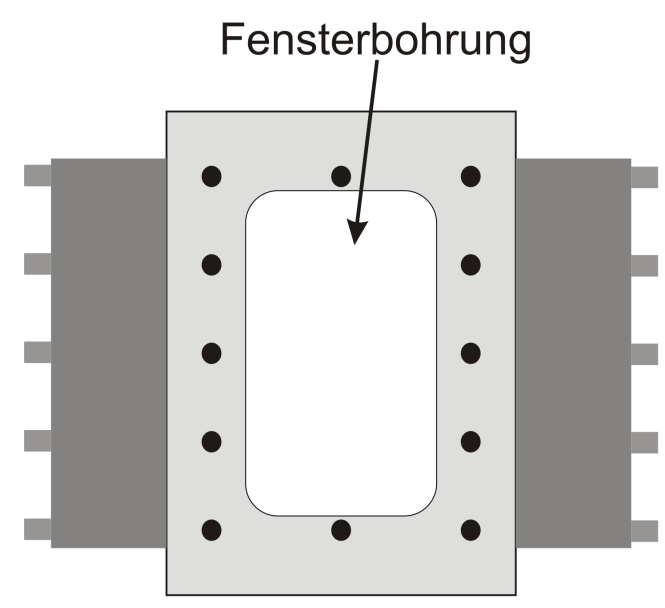

Abbildung 6.1: Schematische Darstellung der Druckkammer. In der Draufsicht befindet sich die mittige Bohrung zur Einkopplung der Sensorsysteme. Die Fensterbohrungen sind auf allen drei Seiten identisch. Am Halterungsflansch sind Gasleitungen, sowie Manometer- und Thermoelementzugänge integriert.

Zur Anregung wurde ein frequenzverdreifachter Nd:YAG-Laser vom Typ Brilliant B von der Firma Quantel verwendet. Dieser arbeitet gepulst (Pulsdauer 5ns) bei einer Frequenz von $10 \mathrm{~Hz}$ und liefert eine maximale Laserenergie von 90mJ. Die Wellenlänge beträgt 355nm und der runde Strahlquerschnitt hat einen Durchmesser von ungefähr $7 \mathrm{~mm}$. Der Laser wurde bei allen Messungen extern angesteuert. Die Triggerung erfolgte bei den Messungen an der Druckkammer mit Hilfe eines digitalen Mehrkanal-Pulsgenerators (Modell DG535, Stanford Research Systems Inc.). Bei den Messungen am Motor wurde eine PTU9-Einheit (Programmable Timing Unit) der Firma LaVision verwendet. Die PTU9 erfasst das $720^{\circ}$ - und das $1^{\circ}$-Triggersignal eines Motors über einen Winkelgeber. Unter Berücksichtigung des zu beobachtenden Kurbelwinkels berechnet dieses Gerät die zeitliche Lage für den Laser- und den Kameratrigger und synchronisiert somit den Messaufbau mit der Motordrehzahl (siehe Abbildung 6.2). 

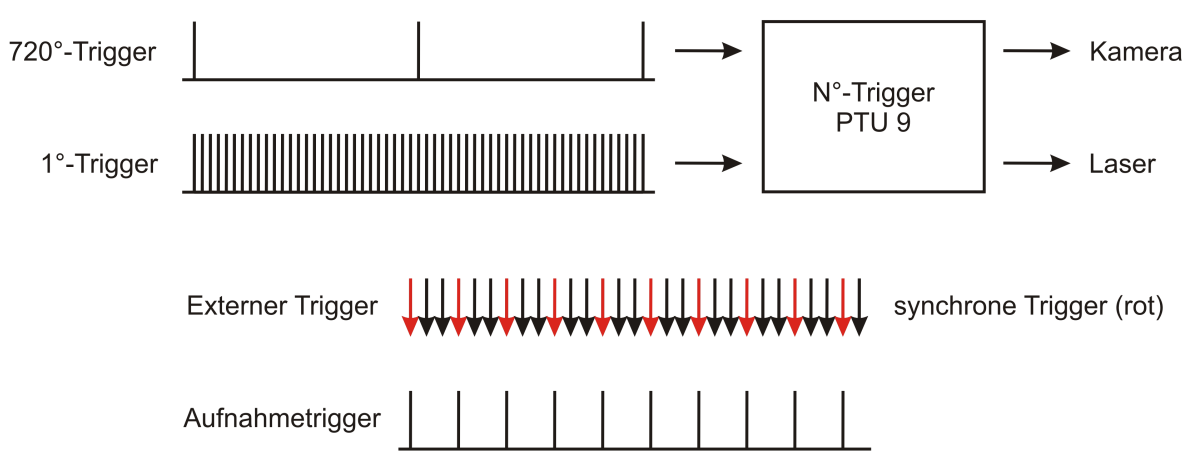

Abbildung 6.2: Triggerschema der Motormessungen. Über einen Kurbelwinkelgeber wird alle $720^{\circ}$ - und $1^{\circ}$-Kurbelwinkel ein Triggerimpuls an die PTU gesendet. Innerhalb der PTU wird eine Synchronisationsfrequenz des zu betrachtenden Kurbelwinkels und dem Kamera-Laser-System berechnet und die Signale werden an die Geräte geleitet. Je nach Drehzahl und Aufnahmefrequenz der Kamera kann ein Kurbelwinkel alle $\mathrm{n}$ Zyklen beobachtet werden. Bestenfalls kann $\mathrm{n}=1$ sein, was aber in den meisten Fällen nicht erreicht werden kann.

Die Kernelemente der Detektionseinheit der meisten Messungen bestehen aus einer bildverstärkten CCD-Kamera und einem Spektrometer. Für die ersten Messungen wurde eine 12bit-CCD-Kamera (FlameStar II, LaVision) mit einer Auflösung von 384x286 Pixel und ein Spektrometer von der Firma L.O.T.-Oriel (MultiSpec, Brennweite $125 \mathrm{~mm}, f / 3,7)$ verwendet. Das für das Spektrometer verwendete Gitter hatte eine Gitterzahl von $\mathrm{G}=2400$ Linien $/ \mathrm{mm}(\mathrm{l} / \mathrm{mm})$. Im Verlauf der Untersuchungen wurde die Detektionseinheit durch Austausch einzelner Komponenten fortwährend optimiert. Für die Verfahrensmessungen wurden Kameras eingesetzt, die aufgrund eines effizienteren Kühlsystems einen stabileren Aufnahmezustand des CCD-Chips ermöglichten und zusätzlich die Aufösung erhöhten. Bei den eingesetzten Geräten handelte es sich um zwei Kameras vom Typ FlameStar III von LaVision mit einer Auflösung von 1100x768 Pixel und 1024x1024 Pixel. Für die Messungen am Motor wurde aufgrund der schnelleren Prozesse eine Kamera mit einem höheren Dynamikbereich gewählt (FlowMaster, 1024x1280 Pixel, 14bit, LaVision). Wie schon erwähnt, handelt es sich um bildverstärkte CCD-Kameras.

Eine spektrale Optimierung des Aufbaus wurde durch den Einsatz des in Kapitel 5.4 vorgestellten Spektrometers von Acton Research erzielt. Hier kam ein Gitter mit einer Gitterzahl von 1200 l/mm zum Einsatz. Abbildung 6.3 skizziert die Grundstruktur des experimentellen Aufbaus. 


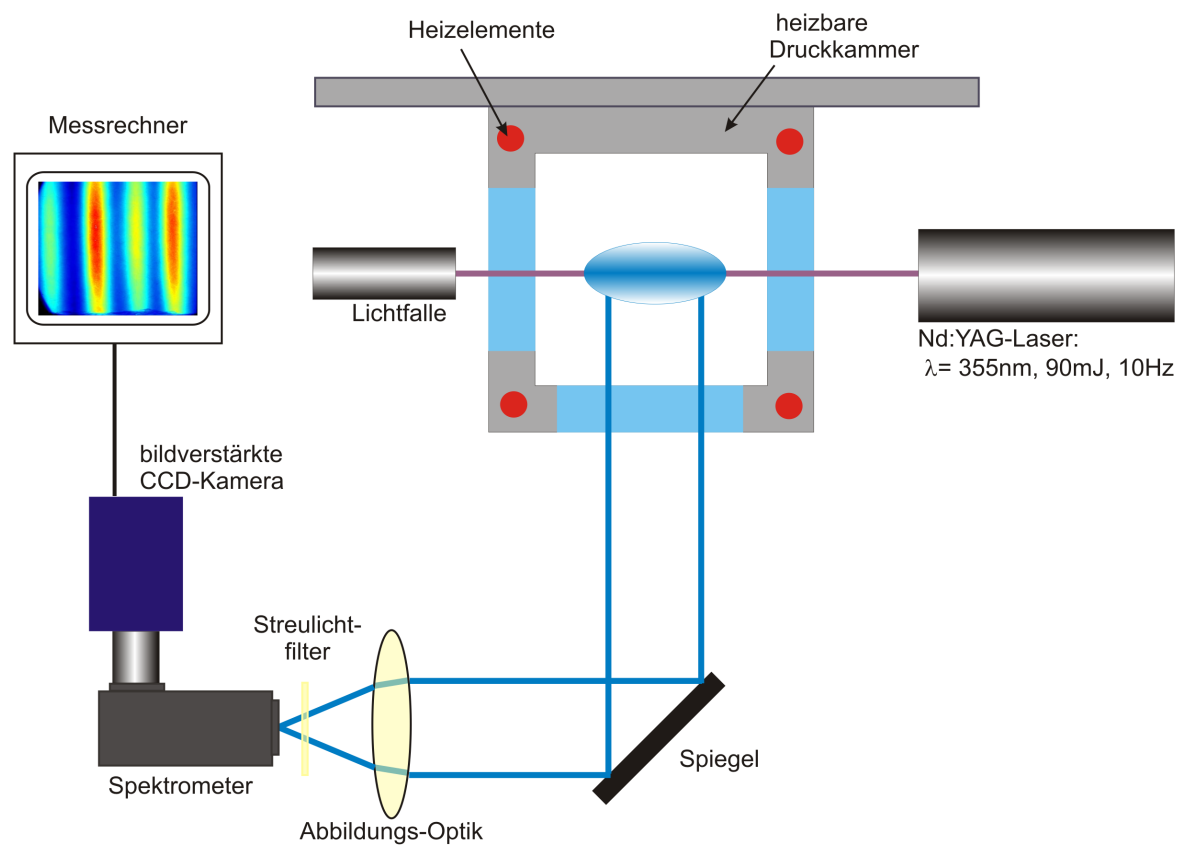

Abbildung 6.3: Schematischer Aufbau der Experimente mit der Druckkammer. Das in der Kammer befindliche Formaldehyd wird mit dem LaserFreistrahl angeregt. Innerhalb der Kammer können motorrelevante Bedingungen geschaffen werden. Mit Hilfe einer F-Zahl angepassten Quarzglaslinse wird das Fluoreszenzsignal in das Spektrometer eingekoppelt.

\subsection{Spektroskopische Voruntersuchungen}

Im ersten experimentellen Abschnitt wurde Formaldehyd auf die für das Verfahren entscheidenden spektroskopischen Größen untersucht. Hier wurde das im LaserLaboratorium entwickelte Laserfluorimeter verwendet, da mit diesem Gerät zeitaufgelöste Fluoreszenzmessungen möglich sind. Das Laserfluorimeter ist ein optischer Vielkanalanalysator und besteht aus einem Diodengepumpten Nd:YAG-Laser, der bei einer emittierenden Wellenlänge von 266nm arbeitet und einem Spektrometer von Jobin Yvon (CP140-103), mit dem der Wellenlängenbereich von 270nm bis $560 \mathrm{~nm}$ detektiert werden kann. Detaillierte Informationen sind in [54] enthalten.

\subsubsection{Fluoreszenzanregung von Formaldehyd}

In diesen spektroskopischen Voruntersuchungen wurde zunächst die Anregbarkeit von Formaldehyd mit dem frequenzverdreifachten Nd:YAG-Laser überprüft, da die- 
ser Übergang, wie in Kapitel 4.5.1 beschrieben, eine schwache Absorption beinhaltet. Daher wurde das in der Druckkammer befindliche Formaldehyd bei unterschiedlichen Temperaturen mit dem frequenzverdreifachten Nd:YAG-Laser $(355 \mathrm{~nm})$ angeregt. Somit diente das Laserfluorimeter nur als Detektor. Das Fluoreszenzsignal wurde über vier Quarzglasfasern mit einem Kerndurchmesser von $400 \mu \mathrm{m}$ erfasst und in das Fluorimeter eingekoppelt.

In einem externen Probenbehälter wurde Paraformaldehyd, welches einen Flammpunkt von $70^{\circ} \mathrm{C}$ besitzt [55], auf eine Temperatur von über $100^{\circ} \mathrm{C}$ erhitzt und somit in den gasförmigen Aggregatzustand gebracht. Dabei entsteht Formaldehyd. In die zuvor evakuierte und auf $100^{\circ} \mathrm{C}$ erhitzte Druckkammer wurde dieses Formaldehyd eingelassen, bis ein Kammerinnendruck von $80 \mathrm{mbar}$ vorlag. Über die optischen Fenster wurde mit Hilfe eines Freistrahls das Formaldehyd angeregt. Die Erfassung des Spektrums wurde bei verschiedenen Temperaturen durchgeführt, um zu prüfen, ob auch bei höheren Temperaturen die Fluoreszenzintensität für die Detektion ausreicht. Abbildung 6.4 zeigt die mit dem Laserfluorimeter gemessenen Spektren bei den Temperaturen $100^{\circ} \mathrm{C}, 120^{\circ} \mathrm{C}$ und $130^{\circ} \mathrm{C}$. Die detektierten Intensitäten erstrecken sich bei einer Temperatur von $100^{\circ} \mathrm{C}$ von 1775 counts $(413 \mathrm{~nm})$ bis 1327 counts (395nm). Dieses Ergebnis zeigt, dass eine Anregung mit dem eingesetzten Nd:YAGLaser hinreichend Fluoreszenzintensität generiert und so das Verfahren mit dem eingesetzten Lasersystem realisierbar ist.

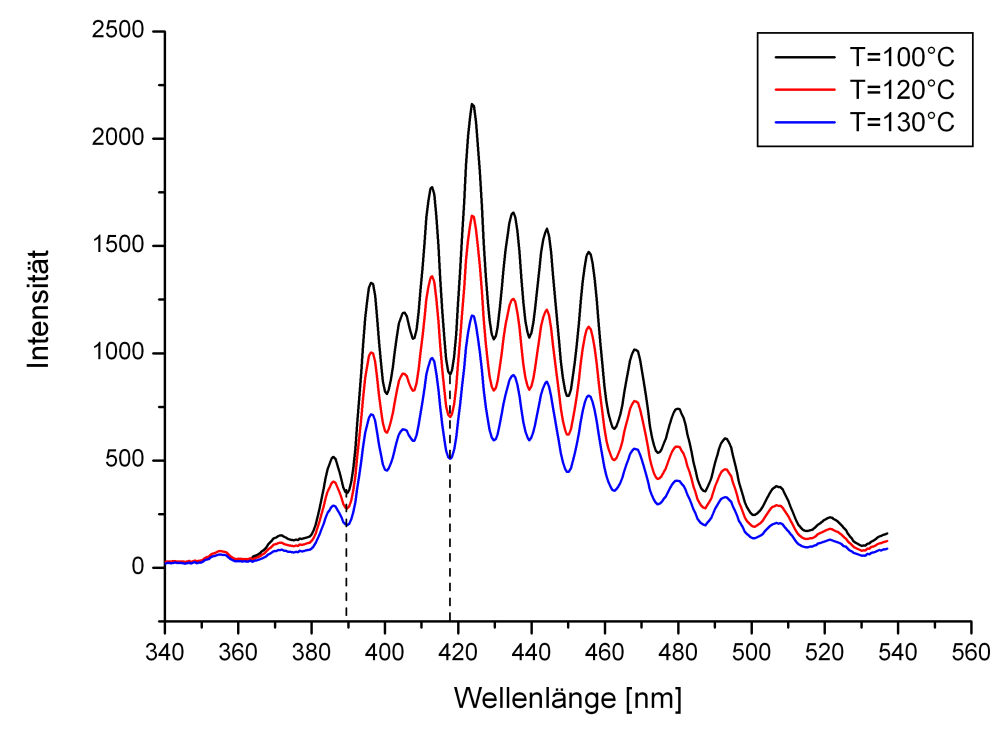

Abbildung 6.4: Emissionsspektrum von Formaldehyd bei unterschiedlichen Temperaturen. Die gestrichelten Linien grenzen den für das Verfahren verwendeten spektralen Bereich ein. 


\subsubsection{Fluoreszenzlebensdauermessungen}

Ein weiterer Hauptgrund für den Einsatz des Laserfluorimeters ist die Möglichkeit der zeitaufgelösten Fluoreszenzmessung. Parallel zur Spektrenerfassung konnte die Fluoreszenzlebensdauer von Formaldehyd gemessen werden. Diese spektroskopische Größe ist insofern von Bedeutung, als dass diese Lebensdauer länger sein muss als die Thermalisierungszeit. Die Fluoreszenz erfährt jedoch durch molekulare Stoßpartner eine Fluoreszenzlöschung (Quenchen), was sich wiederum auf die Fluoreszenzlebensdauer auswirkt. Besonders molekularer Sauerstoff, welcher bei der Verbrennung einen entscheidenden Faktor bildet, ist ein starker Quenching-Partner. Somit wurde der Effekt der Fluoreszenzlöschung auf Formaldehyd untersucht. Synthetische Luft ist ein Gemisch von molekularem Stickstoff und Sauerstoff in einem Verhältnis, wie es in der Atmosphärenluft vorliegt. Die Zusammensetzung besteht aus $80 \% \mathrm{~N}_{2}$ und $20 \% \mathrm{O}_{2}$ [56]. Stickstoff ist ein inertes Gas und hat so gut wie keinen Einfluss auf die Fluoreszenzlebensdauer und kann somit vernachlässigt werden. Innerhalb der Druckkammer wurde ein Partialdruck von 100mbar Formaldehyd erzeugt und mit synthetischer Luft ein Gesamtdruck von 1bar und 10bar geschaffen. Der Druck von 10bar simuliert motorische Bedingungen eines herkömmlichen Ottomotors, der eine Verdichtung von 1:10 erzeugt. In diesen zwei Messreihen wurde die Druckkammer von $100^{\circ} \mathrm{C}$ auf $200^{\circ} \mathrm{C}$ kontinuierlich aufgeheizt und in $10^{\circ} \mathrm{C}$-Abständen die Fluoreszenzlebensdauer gemessen. Tabelle 6.1 und Abbildung 6.5 zeigen die Resultate der beiden Messreihen.

\begin{tabular}{|c|c|c|}
\hline $\begin{array}{c}\text { Temperatur } \\
\mathrm{T}\left[{ }^{\circ} \mathrm{C}\right]\end{array}$ & $\begin{array}{c}\text { Messreihe 1 (1 bar) } \\
\tau[\mathrm{ns}]\end{array}$ & $\begin{array}{c}\text { Messreihe 2 (10 bar) } \\
\tau[\mathrm{ns}]\end{array}$ \\
\hline 100 & 17,33 & 4,58 \\
110 & 16,23 & 4,43 \\
120 & 16,03 & 4,46 \\
130 & 15,54 & 4,12 \\
140 & 15,01 & 4,14 \\
150 & 14,65 & 4,15 \\
160 & 14,38 & 4,05 \\
170 & 13,87 & 3,84 \\
180 & 13,39 & 4,09 \\
190 & 13,24 & 3,9 \\
200 & 12,8 & 3,88 \\
\hline
\end{tabular}

Tabelle 6.1: Ergebnisse der Fluoreszenzlebensdauern von Formaldehyd bei unterschiedlichen Temperaturen und bei Anwesenheit von synthetischer Luft. Bei einem Druck von 1 bar sinkt die Lebensdauer drastisch und hat einen nahezu konstanten Wert von 4 ns.

Wie zu erwarten, ist die kürzeste Lebensdauer bei höchster Temperatur und höchs- 
tem Druck und hat einen Wert von $\tau=3,88 \mathrm{~ns}$. Diese Lebensdauer ist immer noch deutlich länger als die in Kapitel 4.5.1 angegebene Thermalisierungszeit, die sich im ps-Zeitbereich befindet. Somit ist eine weitere Bedingung für das Verfahren erfüllt.

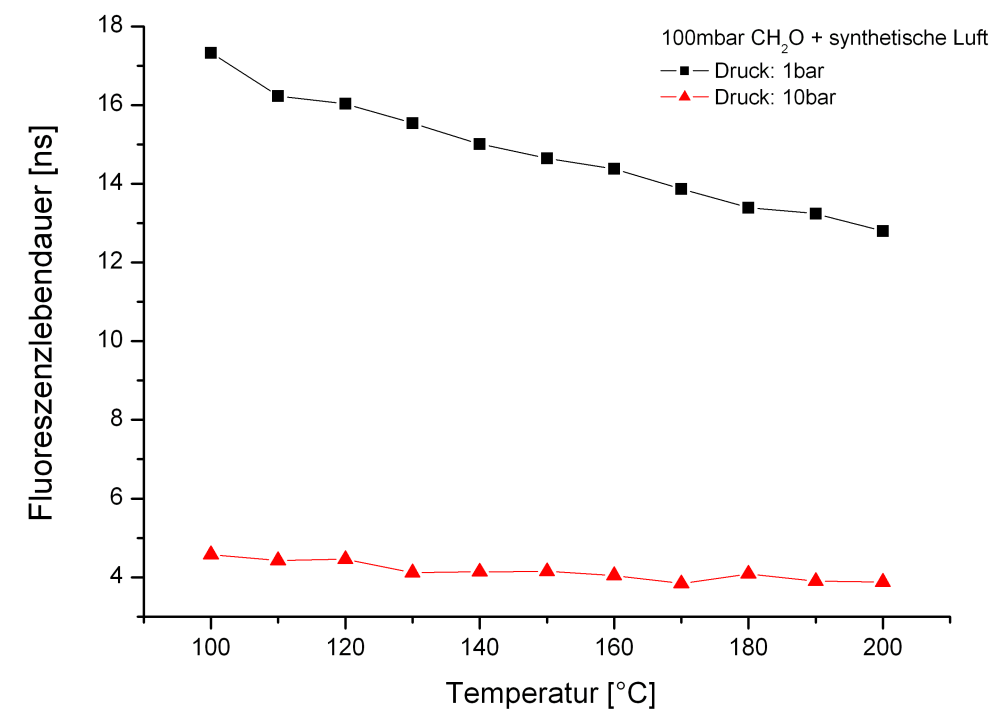

Abbildung 6.5: Fluoreszenzlebensdauern von Formaldehyd bei Anwesenheit von molekularem Sauerstoff. Das Gemisch Formaldehyd/synthetische Luft wurde bei einem Gesamtdruck von 1bar und unter motorrelevanten Bedingungen von 10 bar untersucht. Bei letzterem ist eine nahezu konstante Fluoreszenzlebendauer von $4 \mathrm{~ns}$ zu erkennen.

\subsubsection{Thermalisierung von Formaldehyd}

Die spektroskopischen Untersuchungen ergaben ein positives Resultat bezüglich der Anwendung für das zu entwickelnde Verfahren. Es wurde weiterhin überprüft, ob die Thermaliserung vor allem in den angeregten Zuständen stattgefunden hat, die für die Temperaturbestimmung herangezogen werden. Daher wurde mit dem für die Motormessung relevanten Aufbau der Nachweis der Thermalisierung erfasst. Bei der Erfassung stellt sich weiterhin heraus, welche Kamera-Zeitfenster eingestellt werden müssen, um erst nach Abschluss der Thermalisierung das Fluoreszenzsignal zu detektieren. Die Detektionseinheit besteht aus den Komponenten, die auch bei den Motormessungen Verwendung finden. Erfasst wurde das Signal mit dem Sensor der 2. Generation und mit dem Acton-Spektrometer und der FlowMaster-Kamera verarbeitet. Mit Hilfe eines Engine-Simulators der Firma LaVision wurden die Triggersignale eines Motors bei einer Drehzahl von $\mathrm{n}=995 \mathrm{~min}^{-1}$ simuliert. Der mit Formaldehyd 
vermischte Testkraftstoff Isooktan wurde mit synthetischer Luft in dem verbrennungsrelevanten $\lambda$-Verhältnis von $\lambda=1$ in die Druckkammer bei einem Gesamtdruck von 2 bar eingelassen. Die Temperatur des Gemisches betrug $132,8^{\circ} \mathrm{C}$. Mit Hilfe eines zeitlich sehr kurzen Messfensters von 22 ns wurde entlang der Fluoreszenzabklingzeit das Fluoreszenzsignal abgescannt und das Verhältnis zweier Fluoreszenzlinien gebildet. Der Abschluss der Thermalisierung ist erreicht, wenn dieses Verhältnis konstant bleibt. Aus den vier Verhältniskurven in Abbildung 6.6 wird ersichtlich, dass sich die
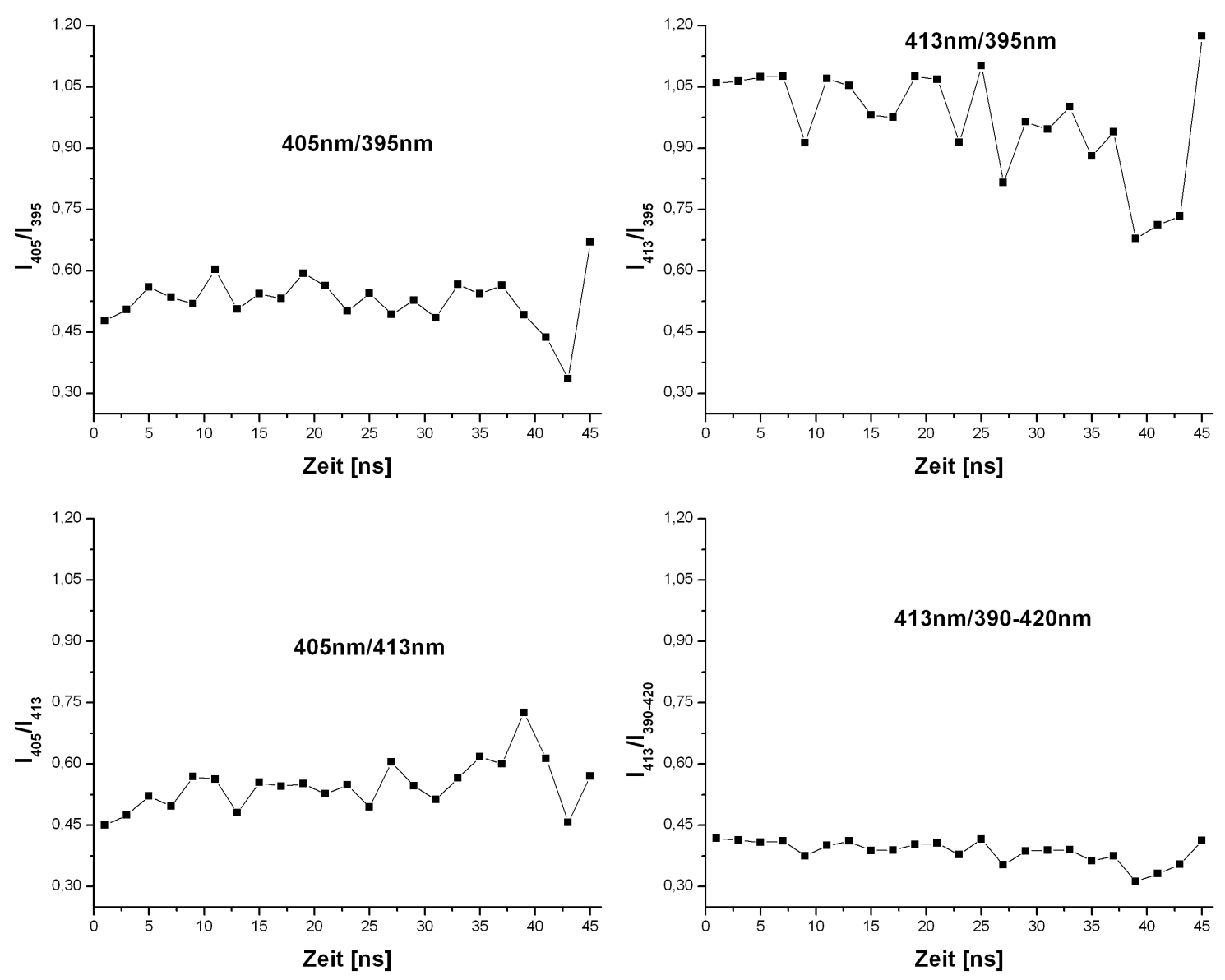

Abbildung 6.6: Überprüfung der Thermalisierung durch unterschiedliche Verhältnisbildungen zweier Fluoreszenzlinien. Am Zeitpunkt $\mathrm{t}=0 \mathrm{~ns}$ setzt der Laserpuls ein. Bei abgeschlossener Thermalisierung bleibt der Linienverhältnis konstant.

Thermalisierung sehr rasch nach dem Laserpuls (in den Kurven bei $t=0$ ns) einstellt. Aufgrund dieser Tatsache kann die Thermalisierung mit dem verwendeten Aufbau nicht zeitlich aufgelöst werden. 


\subsubsection{Fluoreszenzstabilität von Formaldehyd}

Des Weiteren wurde überprüft, wie sich das Fluoreszenzsignal von Formaldehyd unter längerer Einwirkung von Laserbeschuss verhält. Eine Änderung dieses Parameters würde einen negativen Einfluss auf die Temperaturmessung haben und den Messwert verfälschen. Diese Messungen wurden mit der Kamera FlameStar II und dem MultiSpec durchgeführt. In der Druckkammer wurden 50mbar Formaldehyd mit synthetischer Luft zu einem Gesamtdruck von 1bar vermischt. Die Temperatur der Kammer wurde auf $190^{\circ} \mathrm{C}$ konstant gehalten. Der Nd:YAG-Laser beschoss das Gemisch im Freistrahl mit einer Energie von 81mJ. Der Laserbeschuss wurde kontinuierlich bei einer Dauer von 30 Minuten durchgeführt und in 5-Minuten-Abständen die Fluoreszenzintensität bei der Wellenlänge $395 \mathrm{~nm}$ gemessen. Parallel zu diesen Messungen wurde die Energie mit Hilfe eines Energiemessgerätes (LaserMate P) erfasst. Aufgrund der Energieschwankungen des Lasers wurden die Messwerte auf die jeweilige Energie normiert. Um die Werte innerhalb der Messreihe vergleichen zu können, wurden diese wiederum auf den ersten Messpunkt normiert. Die Ergeb-

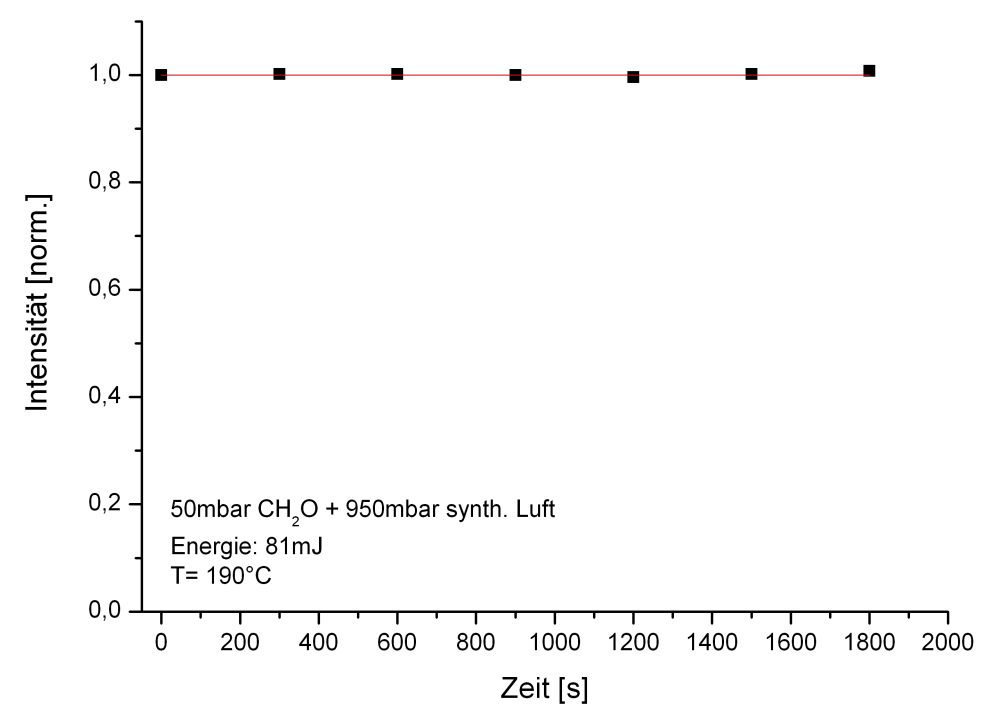

Abbildung 6.7: Normiertes Ergebnis der Fluoreszenzstabilitätsmessungen. Die Messreihe weist nahezu konstante Intensitätswerte auf.

nisse in Abbildung 6.7 zeigen die gute Stabilität des Fluoreszenzsignals hin. Da die Beschussdauer im Verfahren wesentlich kürzer ist als in dem Stabilitätstest, besteht keine Gefahr eines Einflusses durch diese Größe. 


\subsection{Baugruppenspezifische Einflussgrößen}

Für die Entwicklung eines Sensorsystems wurden zuvor einzelne Komponenten daraufhin überprüft, ob spezifische Größen das Messverfahren beeinflussen und für den Einsatz geeignet sind. Neben den in Kapitel 5.5 erbrachten Filtercharakterisierungen lag ein weiterer Schwerpunkt in dem Transmissionsverhalten der verwendeten Quarzglasfasern.

Das Messobjekt war das in Kapitel 5.7.2 beschriebene Faserbündel des Sensorsystems der 2. Generation mit einer Länge von 1,5m. In dieser Untersuchung wurden verschiedene Laserleistungen vor Eintritt in das Faserbündel und direkt beim Austritt am Sensorkopf gemessen. In Abbildung 6.8 sind die Messwerte gegeneinander aufgetragen. Die Resultate der Messung weisen für das Faserbündel eine Transmis-

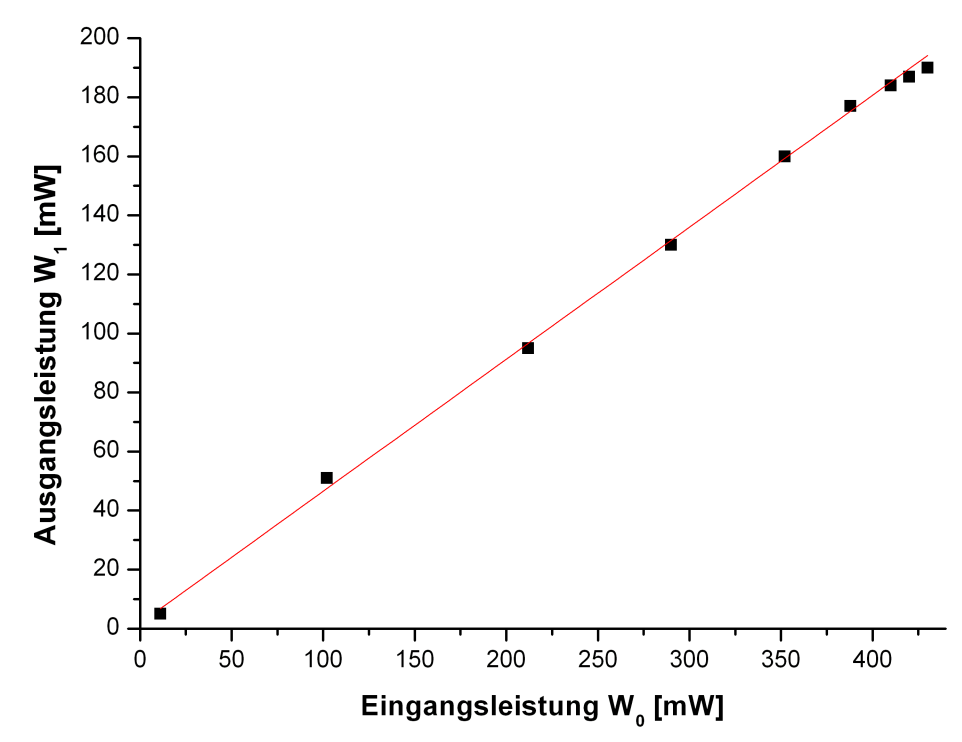

Abbildung 6.8: Transmissionsverhalten des Faserbündels des Sensorsystems der 2. Generation bei unterschiedlichen Eingangsleistungen. Die Steigung der gefitteten Kurve (rot) durch die Messpunkte repräsentiert den Transmissionsfaktor, welcher den Wert von 0,45 besitzt.

sion von $45 \%$ auf. Die relativ hohen Verluste sind jedoch aufgrund der hohen Eingangsleistungen zu erwarten gewesen. Das transmittierte Laserlicht reichte jedoch aus, um eine detektierbare Fluoreszenz erzeugen zu können. Eine positive Erkenntnis dieser Untersuchung war, dass nur sehr geringe, vernachlässigbare Veränderungen der Stirnfläche durch Lasereinkopplung hoher Energie zu erkennen waren. Dies gewährt eine lange Lebensdauer des Faserbündels. Ein Grund dafür ist die recht große Einkoppelfäche, die durch das Verschmelzen mehrerer Fasern mit Hilfe einer Quarz- 
glasplatte realisiert wurde. Damit konnte die Energiedichte verringert werden, bei vollständiger Einkopplung des Laserstrahls.

\subsection{Statische Untersuchung des Verfahrens unter motorrelevanten Umgebungsbedingungen}

Nach Abschluss der spektroskopischen Untersuchungen der Tracersubstanz Formaldehyd und der Anpassung des Messaufbaus auf die verfahrensspezifischen Bedingungen, wurden mit Hilfe der temperierbaren Druckkammer statische Messungen durchgeführt, bei denen motorische Bedingungen vorlagen. Diese Bedingungen beinhalteten Kammerinnendrücke von bis zu 10 bar, was dem typischen Verdichtungsverhältnis eines Ottomotors von 10:1 entspricht. Es wurde Formaldehyd mit dem Testkraftstoff Isooktan vermischt, um mögliche Einflüsse des Kraftstoffes zu untersuchen. Desweiteren sind in der Druckkammer Gemische aus dem tracerangereicherten Kraftstoff mit synthetischer Luft generiert worden. Dabei wurden Gemischzusammensetzungen mit motorrelevanten $\lambda$-Werten erzeugt. Da die Heizstäbe der Kammer eine maximale Kammerinnentemperatur von $200^{\circ} \mathrm{C}$ erzeugen können, wurde im Temperaturbereich von 90 bis $200^{\circ} \mathrm{C}$ gemessen. Dieser Temperaturbereich ist für die Referenzmessungen des entwickelten Sensors ausreichend, da im ungezündeten, geschleppten Motorbetrieb Kompressionstemperaturen von $200^{\circ} \mathrm{C}$ vorliegen. Die gewonnenen Daten wurden zur Kalibrierung der Motormessungen herangezogen.

\subsubsection{Präparation der untersuchten Gemische}

Die für das Verfahren entscheidende Tracersubstanz Formaldehyd wurde, wie auch in den Untersuchungen aus Kapitel 6.2, aus Paraformaldehyd $\left(\mathrm{CH}_{2} \mathrm{O}\right)_{\mathrm{n}}(\mathrm{Merck}$, SigmaAldrich) hergestellt. Diese bei Raumtemperatur in Pulverform vorliegende Substanz wurde in den Voruntersuchungen direkt durch Erhitzen im Probenbehälter in das gasförmige Formaldehyd depolymerisiert und in die Druckkammer geleitet. Für die motorrelevanten Messungen wurde Paraformaldehyd unter Wärmeeinwirkung in Ethanol gelöst. Es wurde eine Löslichkeitsreihe angesetzt, um herauszufinden, welcher maximale Massenanteil von Paraformaldehyd in Ethanol gelöst werden kann. Dabei ergab sich ein maximaler Massenanteil von 30\%. Die in einer gasdicht verschlossenen Laborflasche befindliche Lösung wurde mit Hilfe einer Heizplatte auf eine Temperatur zwischen $130^{\circ} \mathrm{C}$ und $140^{\circ} \mathrm{C}$ gebracht. Das mit Formaldehyd angereicherte Ethanol konnte mit dem Testkraftstoff Isooktan problemlos vermischt werden.

Bei den Messungen sind drei verschiedene $\lambda$-Werte untersucht worden. Es wurden ein mageres $(\lambda=1,5)$, ein fettes $(\lambda=0,5)$ und ein stöchiometrisches Gemisch $(\lambda=1)$ erzeugt. Die prozentualen Volumenanteile der verwendeten Lösung sind 90 Vol.- $\%$ 
Isooktan und 10 Vol.-\% angereichertes Ethanol. Die Stoffmengen der einzelnen Komponenten dieser Lösung ergeben sich durch die folgenden Berechnungen. Ausgehend von einer Lösung mit einem Gesamtvolumen von 100mL ergibt sich für Ethanol eine Stoffmenge von

$$
n_{\mathrm{C}_{2} \mathrm{H}_{5} \mathrm{OH}}=\frac{m_{\mathrm{C}_{2} \mathrm{H}_{5} \mathrm{OH}}}{M_{\mathrm{C}_{2} \mathrm{H}_{5} \mathrm{OH}}}=\frac{6,368 \mathrm{~g} \cdot \mathrm{mol}}{46,07 \mathrm{~g}}=0,14 \mathrm{~mol}
$$

für Formaldehyd erhält man

$$
n_{\mathrm{CH}_{2} \mathrm{O}}=\frac{m_{\mathrm{CH}_{2} \mathrm{O}}}{M_{\mathrm{CH}_{2} \mathrm{O}}}=\frac{2,652 \mathrm{~g} \cdot \mathrm{mol}}{30,03 \mathrm{~g}}=0,09 \mathrm{~mol}
$$

und für Isooktan

$$
n_{\mathrm{C}_{8} \mathrm{H}_{18}}=\frac{m_{\mathrm{C}_{8} \mathrm{H}_{18}}}{M_{\mathrm{C}_{8} \mathrm{H}_{18}}}=\frac{62,1 \mathrm{~g} \cdot \mathrm{mol}}{114,23 \mathrm{~g}}=0,54 \mathrm{~mol} .
$$

Im Nenner steht die molare Masse $M$ der jeweiligen Stoffe.

Für die Berechnung der $\lambda$-Werte innerhalb der Kammer wird von einer stöchiometrischen Reaktionsgleichung ausgegangen, deren Reaktion eine vollständige Verbrennung von Isooktan $\left(\mathrm{C}_{8} \mathrm{H}_{18}\right)$, Ethanol $\left(\mathrm{C}_{2} \mathrm{H}_{5} \mathrm{OH}\right)$ und Formaldehyd $\left(\mathrm{CH}_{2} \mathrm{O}\right)$ darstellt. Schematisch handelt es sich um folgende Form:

$$
\alpha \mathrm{C}_{8} \mathrm{H}_{18}+\beta \mathrm{CH}_{2} \mathrm{O}+\gamma \mathrm{C}_{2} \mathrm{H}_{5} \mathrm{OH}+\delta \mathrm{O}_{2} \rightarrow \epsilon \mathrm{CO}_{2}+\zeta \mathrm{H}_{2} \mathrm{O}
$$

Unter Berücksichtigung der oben ermittelten Stoffmengen ergeben sich folgende Reaktionen.

Ethanol:

$$
0,14\left[\mathrm{C}_{2} \mathrm{H}_{5} \mathrm{OH}+3 \mathrm{O}_{2} \rightarrow 2 \mathrm{CO}_{2}+3 \mathrm{H}_{2} \mathrm{O}\right]
$$

Formaldehyd:

$$
0,09\left[\mathrm{CH}_{2} \mathrm{O}+\mathrm{O}_{2} \rightarrow \mathrm{CO}_{2}+\mathrm{H}_{2} \mathrm{O}\right]
$$

Isooktan:

$$
0,54\left[\mathrm{C}_{8} \mathrm{H}_{18}+12,5 \mathrm{O}_{2} \rightarrow 8 \mathrm{CO}_{2}+9 \mathrm{H}_{2} \mathrm{O}\right]
$$

Durch Multiplikation der Stoffmengen folgt die resultierende Reaktion:

$$
0,54 \mathrm{C}_{8} \mathrm{H}_{18}+0,09 \mathrm{CH}_{2} \mathrm{O}+0,14 \mathrm{C}_{2} \mathrm{H}_{5} \mathrm{OH}+7,23 \mathrm{O}_{2} \rightarrow 4,67 \mathrm{CO}_{2}+5,34 \mathrm{H}_{2} \mathrm{O}
$$

Anstelle von reinem Sauerstoff wurde synthetische Luft zur Befüllung der Kammer verwendet. Es wurde somit ein Sauerstoffanteil von 21\% in der Luft angenommen 
(entspricht einem Anteilfaktor von 1/4,76). Aufgrund der Stoffmenge des Sauerstoffs von $n_{O_{2}}=7,23$ ergibt sich für Luft eine Stoffmenge von

$$
n_{\text {Luft }}=7,23 \cdot 4,76=34,42 .
$$

Die Gemischzusammensetzung wird über den Kammerdruck reguliert. Das KraftstoffLuft-Verhältnis $\lambda$ kann über den Druck durch

$$
\lambda=\frac{p(\text { Gemisch })}{p(\text { Luft })}
$$

ausgedrückt werden. Für die Reaktion R8 ist der $\lambda$-Wert mit folgender Formel definiert

$$
\frac{p\left(\mathrm{C}_{8} \mathrm{H}_{18}\right)+p\left(\mathrm{CH}_{2} \mathrm{O}\right)+p\left(\mathrm{C}_{2} \mathrm{H}_{5} \mathrm{OH}\right)}{p(\text { Luft })}=\frac{n_{\mathrm{C}_{8} \mathrm{H}_{18}} \cdot \frac{R T}{V}+n_{\mathrm{CH}_{2} \mathrm{O}} \cdot \frac{R T}{V}+n_{\mathrm{C}_{2} \mathrm{H}_{5} \mathrm{OH}} \cdot \frac{R T}{V}}{n_{L u f t} \cdot \frac{R T}{V}}
$$

Da der Faktor $\frac{R T}{V}$ für alle Substanzen identisch ist, vereinfacht sich $6.10 \mathrm{zu}$

$$
\frac{n_{\mathrm{C}_{8} \mathrm{H}_{18}}+n_{\mathrm{CH}_{2} \mathrm{O}}+n_{\mathrm{C}_{2} \mathrm{H}_{5} \mathrm{OH}}}{n_{\text {Luft }}}=\frac{0,54+0,09+0,14}{34,42}=\frac{0,77}{34,42} .
$$

Somit gilt für den $\lambda$-Werte bei verschiedenen Drücken:

$$
\lambda=\frac{p(\text { Luft })}{p(\text { Gemisch })} \cdot \frac{1}{44,7}
$$

Bei gegebenem Luftdruck $p(L u f t)$ und $\lambda$-Wert kann der einzustellende Gemischdruck $p($ Gemisch $)$ berechnet werden:

$$
p(\text { Gemisch })=\frac{p(\text { Luft })}{\lambda \cdot 44,7}
$$

\subsubsection{Auswertung der Messergebnisse}

Mit Hilfe einer bildverstärkten CCD-Kamera wurden die Spektren von Formaldehyd aufgenommen. Bei den statischen Untersuchungen an der Druckkammer stellt das gespeicherte Spektrum den Mittelwert von zehn Spektren dar. Jedes dieser zuletzt genannten Spektren besteht wiederum aus zehn Einzelaufnahmen, die mittels OnChip-Integration erfasst worden sind. Die Mittelung über insgesamt 100 Spektren wurde aufgrund der Kalibration für die Motormessungen gewählt. Für die Motormessungen wurden Einzelaufnahmen ohne On-Chip-Integration verwendet.

Die Auswertung der Spektren erfolgte mit Hilfe eines Algorithmus, der über ein Makro in der Bildverarbeitungssoftware DaVis von der Firma LaVision ausgeführt wurde. Mit Hilfe der in Kapitel 4.5.1 abgeleiteten Arrhenius-Funktion (4.27) wird 
über die Besetzung der einzelnen Energieniveaus die Temperatur abgefragt. Ausgangspunkt dieser Erfassung ist die Gleichung

$$
\ln \left(\frac{F_{1}}{F_{2}}\right)=-\frac{\Delta E}{k_{B} T}
$$

Die Population der Niveaus kann über die Fluoreszenzintensitäten der jeweiligen Wellenlänge erfasst werden. Dabei wird durch Integration der spektral aufgelösten Übergangslinie die entsprechende Intensität bestimmt. Es stellte sich heraus, dass schon eine geringe Variation der Integrationsgrenzen einen starken Einfluss auf das Verhältnis hatte und für das empfindliche Verfahren eine große Streuung der Temperaturwerte die Folge war. Daher musste vor der eigentlichen Auswertung eine Glättung des Spektrums erfolgen. Unter der Anwendung verschiedener Glättungsfunktionen ergab der gleitende Mittelwert die besten Resultate. Hierbei werden in einer Datenreihe vorhandene Variationen dahingehend verringert, dass ein arithmetischer Mittelwert von aufeinanderfolgenden Datenpunkten gebildet wird. Der zentrierte, gleitende Mittelwert der Ordnung $\mathrm{n}=3$ würde bei den Datenpunkten $y_{1}$, $y_{2}, y_{3}, \ldots$ durch

$$
\bar{y}_{2}=\frac{y_{1}+y_{2}+y_{3}}{3}, \quad \bar{y}_{3}=\frac{y_{2}+y_{3}+y_{4}}{3}, \ldots
$$

berechnet werden. Für eine ungeradzahlige Ordnung n gilt allgemein die Folge

$$
\begin{aligned}
\bar{y}_{\frac{n+1}{2}} & =\frac{y_{1}+y_{2}+\ldots+y_{\frac{n+1}{2}}+\ldots+y_{n}}{n}, \\
\bar{y}_{\frac{n+1}{2}+1} & =\frac{y_{1}+y_{2}+\ldots+y_{\frac{n+1}{2}+1}+\ldots+y_{n+1}}{n} \ldots
\end{aligned}
$$

$\mathrm{Zu}$ beachten ist, dass für die ersten und letzten $\frac{n-1}{2}$ Datenpunkte kein gleitender Durchschnittswert vorliegt. Da der Spektralbereich des aufgenommenen Spektrums größer als der benötigte Bereich ist, stellt dies kein Problem für die Auswertung dar. 


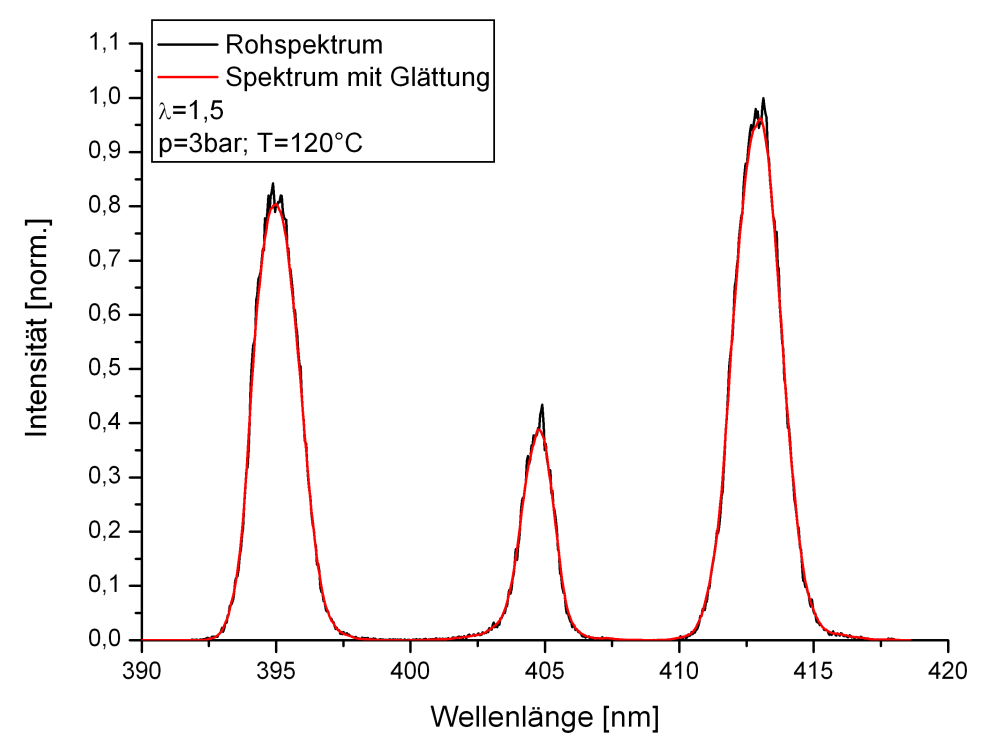

Abbildung 6.9: Glättung der aufgenommenen Spektren. Das Rohspektrum (schwarz) wurde von einem Kraftstoff-Luft-Gemisch mit $\lambda=1,5$ bei einem Druck von 3bar und einer Temperatur von $120^{\circ} \mathrm{C}$ aufgenommen.

Abbildung 6.9 zeigt das Spektrum ohne Glättung (schwarz) und das geglättete Spektrum (rot). Die Aufnahme wurde unter motorischen Bedingungen an der Druckkammer durchgeführt. Es wurde der für die Motormessungen konzipierte Sensoraufbau verwendet und dieser mit Hilfe des Engine-Simulators bei einer eingestellten Drehzahl von 1000 $\mathrm{min}^{-1}$ getriggert. In Abbildung 6.10 verdeutlichen die Graphen die Empfindlichkeit des Verfahrens ohne und mit Glättung des Spektrums. Die Arrhenius-Funktion ist durch die orange Linie dargestellt, während die gestrichelten Linien den oberen und unteren Toleranzbereich verdeutlichen. Um eine Einschätzung zu bekommen, wie empfindlich das Verfahren gegenüber der Variation der Integrationsgrenzen ist, wurden bestimmte Messpunkte für die Analyse herangezogen. Die Untersuchung wurde an Messungen vorgenommen, bei denen ein KraftstoffLuft-Verhältnis von $\lambda=1$ und ein Druck von 2bar vorlag. Das aus nicht geglätteten Spektren generierte Verhältnis 405nm/413nm lieferte die Messpunkte für die Arrhenius-Kurve. Dabei wurden Messpunkte aus einem niedrigen $\left(103-108^{\circ} \mathrm{C}\right)$ und einem hohen Temperaturbereich $\left(123-125^{\circ} \mathrm{C}\right)$ genommen. Innerhalb dieses Temperaturbereichs wurden drei Messpunkte gewählt, die sowohl auf dem Arrhenius-Fit als auch auf dem oberen und unteren Toleranzbereich liegen. Nach Anwendung der oben beschriebenen Glättungsfunktion wurde eine deutlich reduzierte Streuung erzielt und die Empfindlichkeit des Verfahrens auf Variation der Integrationsgrenzen ver- 
ringert. Nach Glättung des Spektrums werden die Fluoreszenzlinien von den spektralen Hintergrundsignalen getrennt. Dies ist eine molekülspezifische Größe und nicht zu verwechseln mit dem Signalrauschen des CCD-Chips oder eventuell vorhandenes Streulicht. Diese Signale werden während der Messung durch ein Dunkelbild erfasst und dieses von dem aufgenommenen Spektrum abgezogen. Zuletzt findet die eigentliche Bildung des Verhältnisses statt. Der Quellcode dieses Auswerte-Algorithmus ist in Anhang B im Detail dargestellt. 

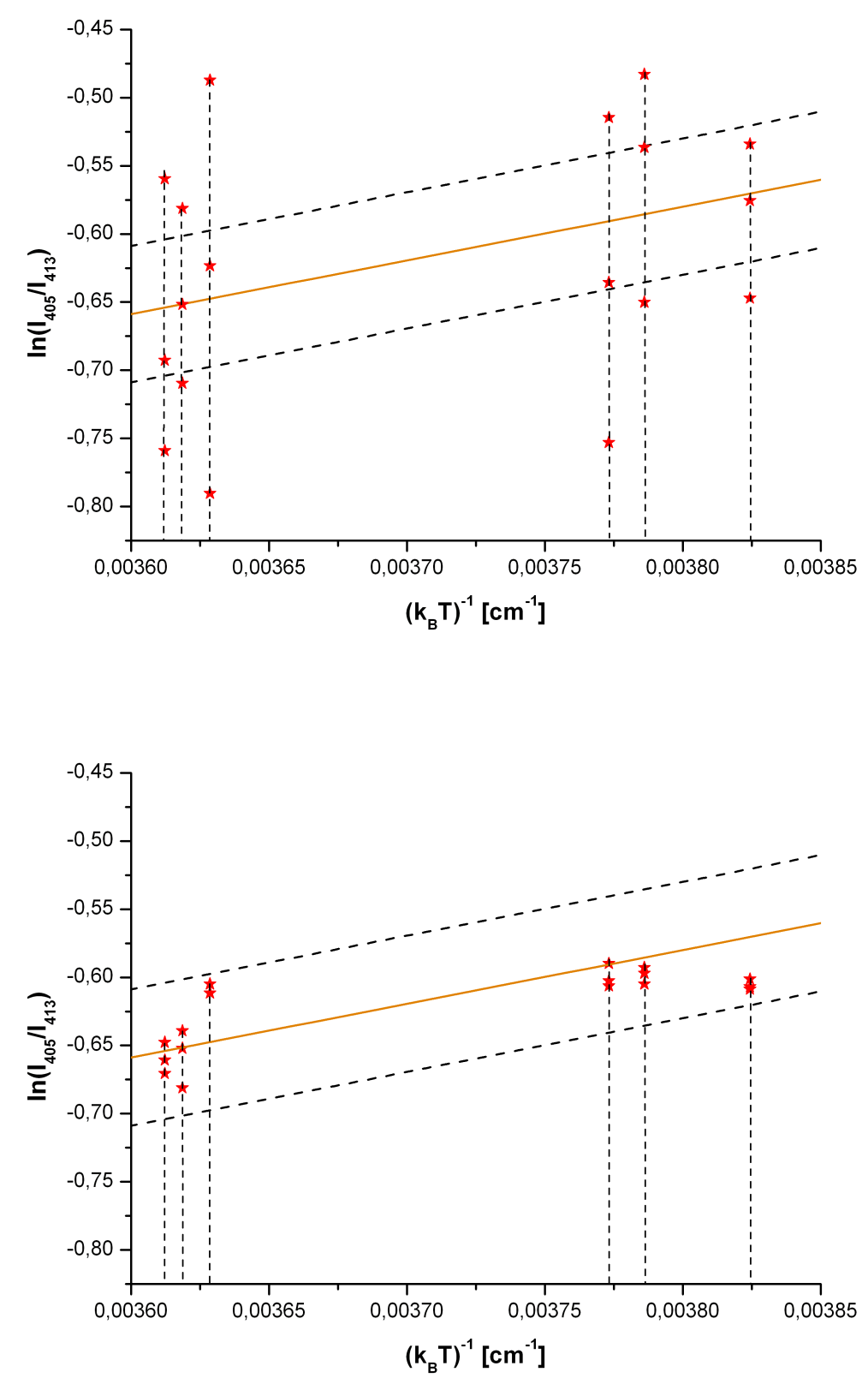

Abbildung 6.10: Einfluss bei Variation der Integrationsgrenzen auf die Verhältnisbildung. Die orange Linie zeigt den Fit der Arrhenius-Funktion. Die gestrichelten Linien repräsentieren die obere und untere Toleranzgrenze. Im oberen Graphen sind die berechneten Verhältnisse ohne Glättung des Spektrums zu sehen, im unteren mit Anwendung einer Glättung. 


\subsubsection{Statische Messungen an der Druckkammer}

Mit Hilfe der in Kapitel 6.1 beschriebenen Experimente an der Druckkammer konnten LIF-Messungen unter statischen Bedingungen durchgeführt werden. Dies hat den Vorteil, mit Hilfe eines relativ unkomplizierten Aufbaus eine Einschätzung über das Verfahren zu bekommen. Die Kammer ist so konzipiert, dass motornahe Umgebungsbedingungen geschaffen werden können. Somit wurden neben den Machbarkeitsstudien auch die für die Temperaturbestimmung notwendigen Kalibrationsmessungen durchgeführt. Des Weiteren sind Testmessungen mit den entwickelten Sensoren an der Kammer realisiert worden. Die Laserenergie der Kammermessungen betrug zwischen 80 und $90 \mathrm{~mJ}$.

In den ersten Messungen wurde mit einem Formaldehyd/Luft-Gemisch bei unterschiedlichen Drücken gearbeitet. In die auf $90^{\circ} \mathrm{C}$ vorgeheizte Kammer wurden $100 \mathrm{mbar}$ Formaldehyd eingeleitet und mit Hilfe von synthetischer Luft auf einen definierten Kammerinnendruck gebracht. Die Messreihen für einen Gesamtdruck von 1 bis 10 bar wurden in 1bar-Schritten nacheinander durchgeführt. Zusätzlich wurde eine nur aus 100mbar Formaldehyd bestehende Probe gemessen. Die Messungen wurden mit der FlameStar II und dem MultiSpec durchgeführt. Während der kontinuierlichen Aufnahme der Spektren wurde das Gemisch von $90^{\circ} \mathrm{C}$ auf $200^{\circ} \mathrm{C}$ stetig aufgeheizt und zu jeder Aufnahme der Temperaturwert notiert. Die Darstellung der Temperaturinformation erfolgte wie im vorherigen Kapitel durch die logarithmische Auftragung der Arrheniusfunktion basierend auf Gleichung 6.15. In Abbildung 6.11 sind alle möglichen Verhältnisse der drei erfassten Übergänge abgebildet. Es stellte sich heraus, dass das Verhältnis 405nm/413nm die geringsten Querempfindlichkeiten bezüglich des Drucks und der Konzentration aufwies. 

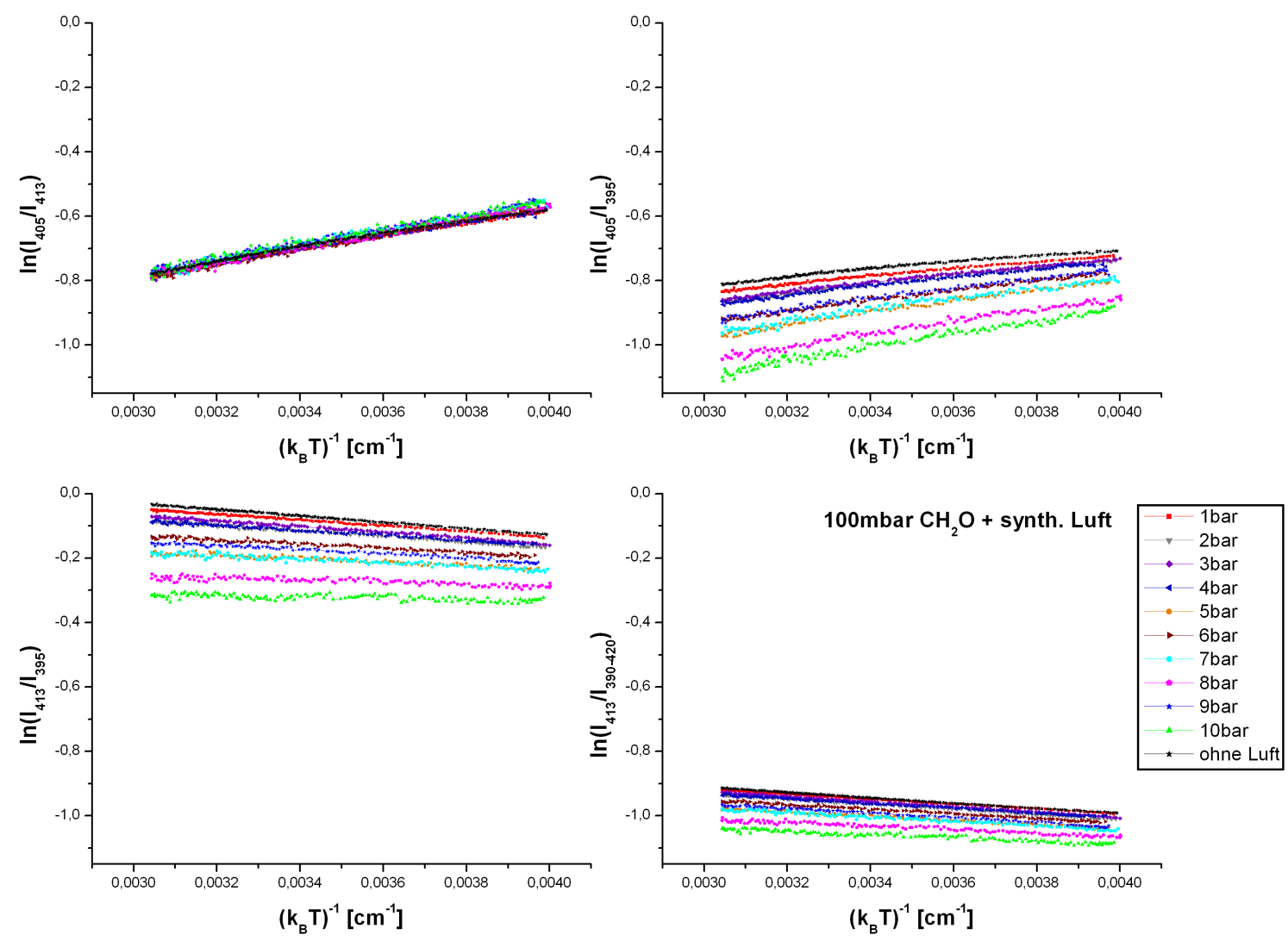

Abbildung 6.11: Logarithmische Auftragung der Arrheniusfunktion der Messungen von Formaldehyd/Luft-Gemischen. Es ist der Druckbereich 1 bis 10bar mit verschiedenen Konzentrationen untersucht worden. Die Kammer wurde im Temperaturbereich von $90^{\circ} \mathrm{C}$ bis $200^{\circ} \mathrm{C}$ betrieben.

Aufgrund der motorischen Applikation wurde der Testkraftstoff Isooktan mit Formaldehyd angereichert. Mit diesem Kraftstoff wurden in der Kammer erste motorisch relevante Kraftstoff-Luft-Gemische erzeugt, um mögliche Einflüsse des Isooktans auf die Fluoreszenz aufzudecken. Die Ergebnisse in Abbildung 6.12 wurden von Proben mit einem Gesamtdruck von jeweils 10 bar erzielt. Die Messreihen hatten die $\lambda$-Werte $\lambda_{1}=0,6, \lambda_{2}=1,1$ und $\lambda_{3}=1,7$. Zum Vergleich ist der Arrheniusplot der zuvor erbrachten Messung mit Formaldehyd bei 1bar in der Grafik eingetragen. Die Daten weisen keinen Einfluss des Isooktans auf die Fluoreszenz des Formaldehyds auf. 


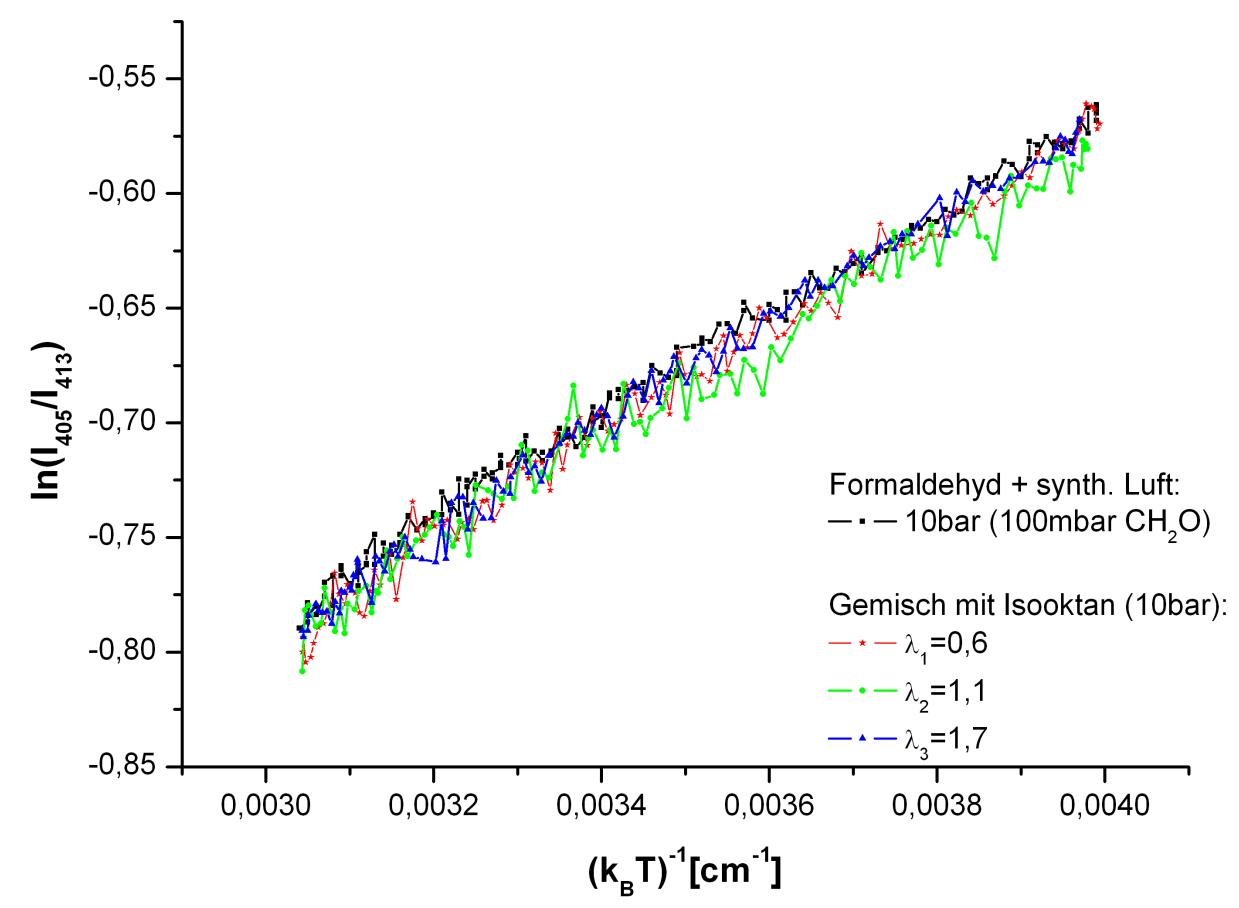

Abbildung 6.12: Exemplarische Arrheniusauftragung des Verhältnisses $405 \mathrm{~nm} / 413 \mathrm{~nm}$ bei motorrelevanten $\lambda$-Werten. Alle drei Kurven weisen einen nahezu identischen Verlauf auf. Die Übereinstimmung mit der Messung von Formaldehyd/Luft deutet auf keinerlei Einflussnahme des Isooktans hin.

Nachdem kein Einfluss des Kraftstoffes festgestellt wurde, sind Messreihen mit definierten $\lambda$-Werten bei verschiedenen Druckbereichen gemacht worden. Auch hier kristallisierte sich das Linien-Verhältnis $405 \mathrm{~nm} / 413 \mathrm{~nm}$ als jenes heraus, welches die geringsten Querempfindlichkeiten aufwies. In den Abbildungen 6.13 bis 6.15 sind die Resultate für die Drücke 1, 2, 4, 6, 8 und 10 bar zu sehen. Es wurden drei Gemische mit den Werten $\lambda_{1}=0,5, \lambda_{2}=1$ und $\lambda_{3}=1,5$ generiert.

Die geringe Querempfindlichkeit des Linienverhältnisses 405nm/413nm in den Formaldehyd/Luft-Gemischen konnte auch unter Anwesenheit des Testkraftstoffes Isooktan bei verschiedenen $\lambda$-Werten reproduziert werden. Bei den Messungen mit Testkraftstoff ist in den Abbildungen 6.13 bis $6.15 \mathrm{zu}$ sehen, dass auch die Verhältnisse $413 \mathrm{~nm} / 395 \mathrm{~nm}$ und vor allem 413nm/390-420nm einen stabilen Verlauf haben und für Druck- und Konzentrationsschwankungen unempfindlicher sind. Ein weiterer wichtiger Aspekt ist die größere Steigung des Verhältnisses 405nm/413nm. Diese Tatsache wirkt sich positiv auf die Temperatursensitivität des Messverfahrens aus. 

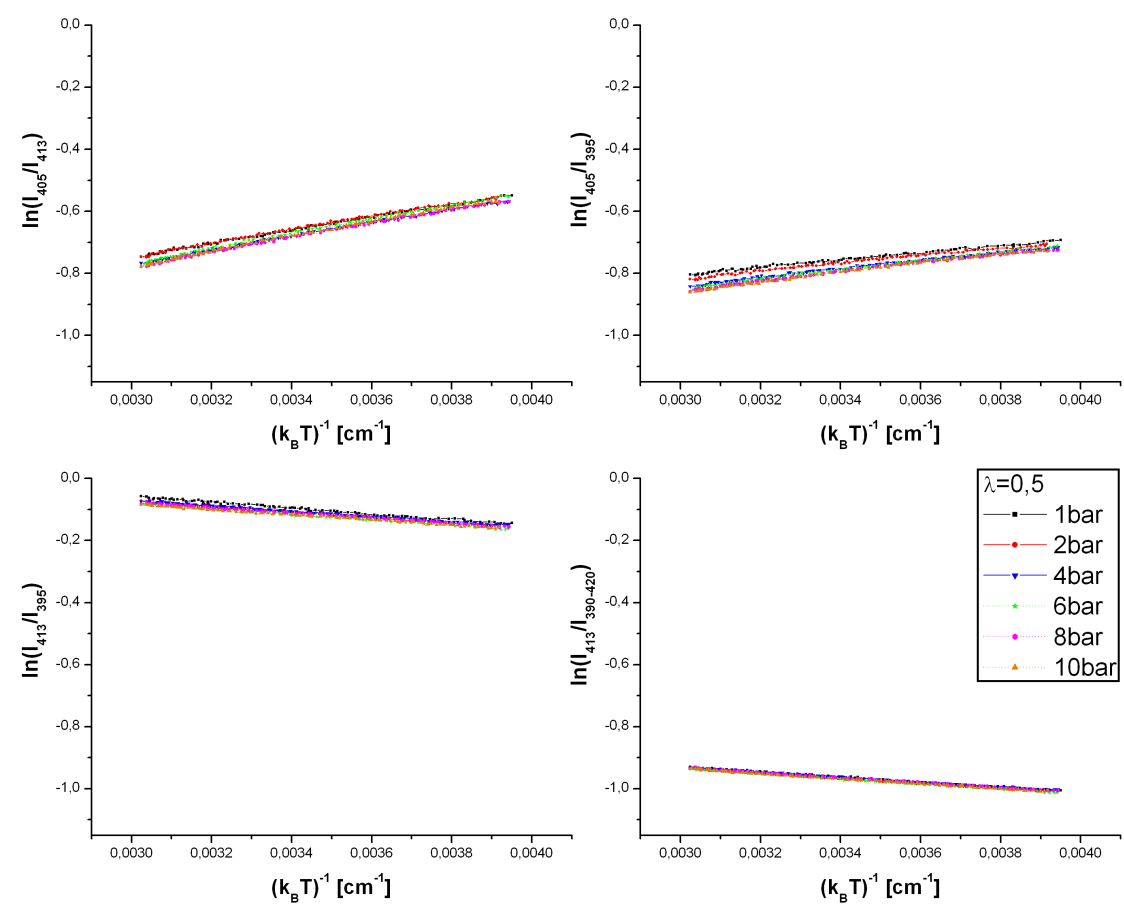

Abbildung 6.13: Arrheniusauftragung der drei detektierten Fluoreszenzlinien für $\lambda=0,5$.
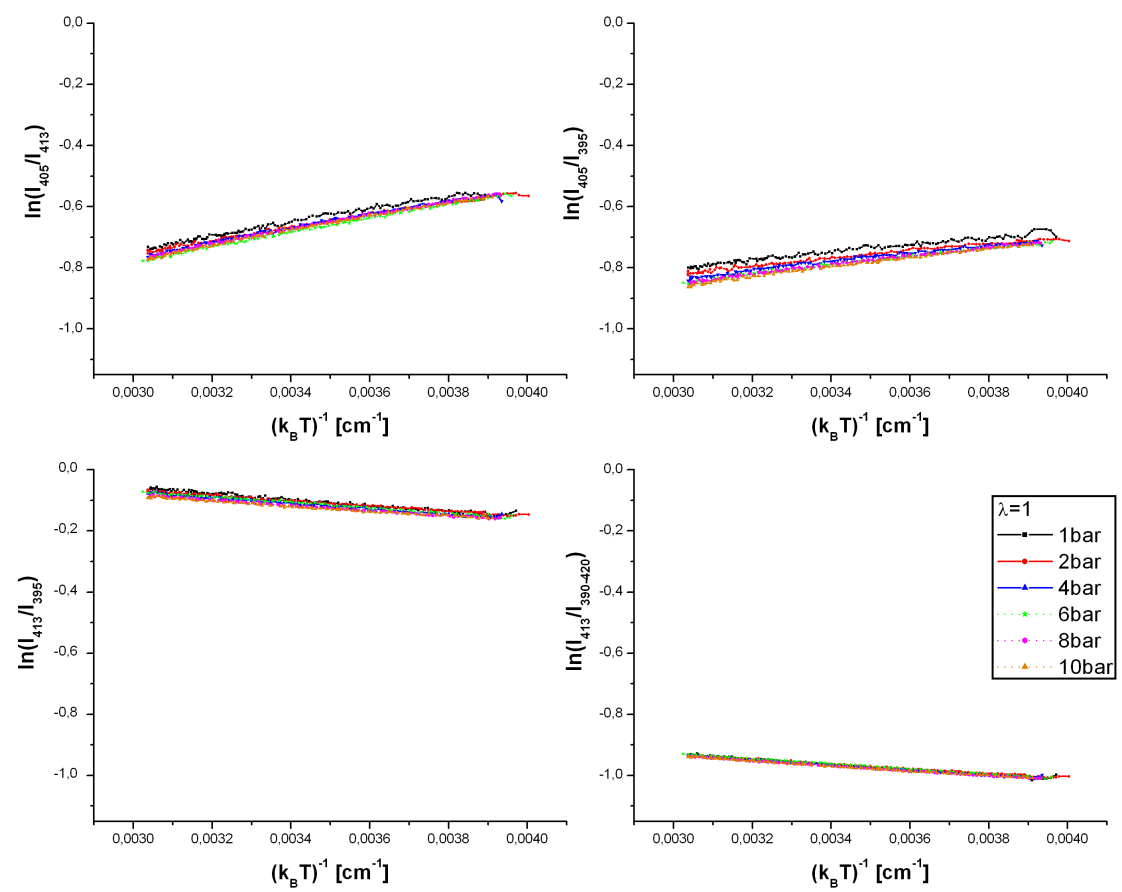

Abbildung 6.14: Arrheniusauftragung der drei detektierten Fluoreszenzlinien für $\lambda=1$. 

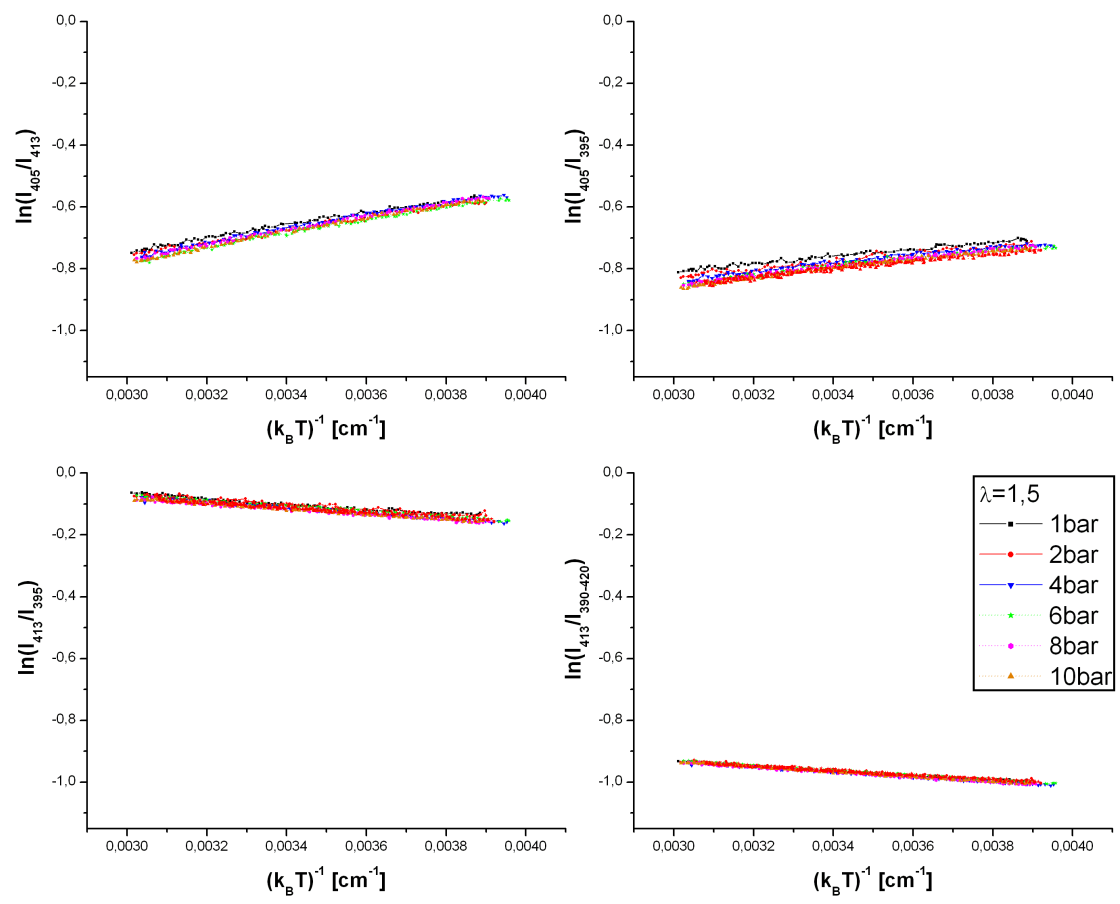

Abbildung 6.15: Arrheniusauftragung der drei detektierten Fluoreszenzlinien für $\lambda=1,5$.

\subsubsection{Sensormessungen an der Druckkammer}

Es wurde versucht, die zuvor erbrachten Resultate mit Hilfe eines Sensorsystems zu reproduzieren und das Verfahren für die motorische Applikation anwendbar zu machen. Dafür wurde der in Kapitel 5.7.1 beschriebene Sensor entwickelt. Im nächsten Schritt wurden die mit dem ersten Sensor gemachten Erfahrungen für eine Weiterentwicklung eines zweiten Prototyps genutzt. Beide Sensoren wurden an der Druckkammer getestet, die mit Hilfe eines Adapterflansches eingelassen wurden (siehe Abbildung 6.16). Aus Sicherheitsgründen wurde bei den Sensormessungen mit niedrigen Drücken gearbeitet. Auch der Temperaturbereich wurde auf maximal $150^{\circ} \mathrm{C}$ beschränkt. Dies liegt darin begründet, dass die Kammer nicht über eine kontrollierbare Kühlung besitzt und bei längeren Messungen der Sensor erhitzt. Die für die Sensoren verwendeten Epoxydharz-Klebstoffe haben eine Temperaturbeständigkeit von maximal $200^{\circ} \mathrm{C}$. Mit der Einschränkung soll eine Verringerung der Klebeeigenschaften verhindert werden. Der Einsatz am Motor hingegen ist problemlos, da der Motorblock einen Kühlkreislauf besitzt, der auf eine Temperatur von $90^{\circ} \mathrm{C}$ herunterkühlen soll. 

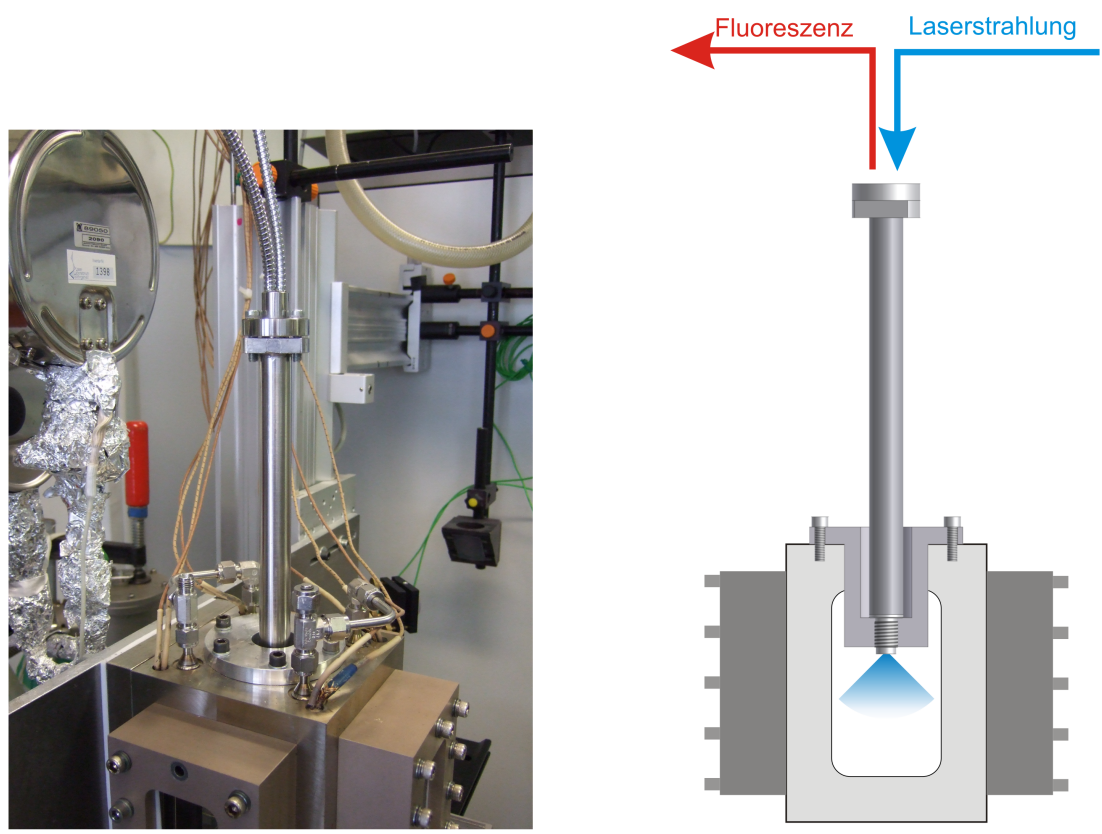

Abbildung 6.16: Druckkammer mit integrierten Sensor (Im Bild ist der weiterentwickelte Sensor zu sehen). Rechts: Schema zur Sensormessung in der Druckkammer.

\section{Messungen mit dem Sensorsystem der 1. Generation}

Der erste Sensor wurde bei einem Kammerinnendruck von 1bar und im Temperaturbereich von $100^{\circ} \mathrm{C}$ bis $150^{\circ} \mathrm{C}$ getestet. Das untersuchte Medium war ein Gemisch aus 100mbar Formaldehyd und synthetischer Luft. Die Lasereinkopplung erfolgte über einen Freistrahl direkt in den Sensorkopf, um relativ viel Laserenergie einzukoppeln. Ein Schema des Experimentes ist in Abbildung $6.17 \mathrm{zu}$ erkennen.

Innerhalb der Testmessungen dieses Sensortyps erfolgte aufgrund einer Fokusbildung durch die Mantelfläche des Quarzglasstabes eine Zerstörung der reflektierenden Umlenkfläche. Dennoch konnten erste Messergebnisse erzielt werden, welche eine Aussage über die Realisierbarkeit eines sensorbasierten Messverfahrens zugelassen haben. In Abbildung 6.18 sind die Sensordaten den Messungen ohne Sensor bei gleichem Druck und $\lambda=1$ gegenübergestellt. 


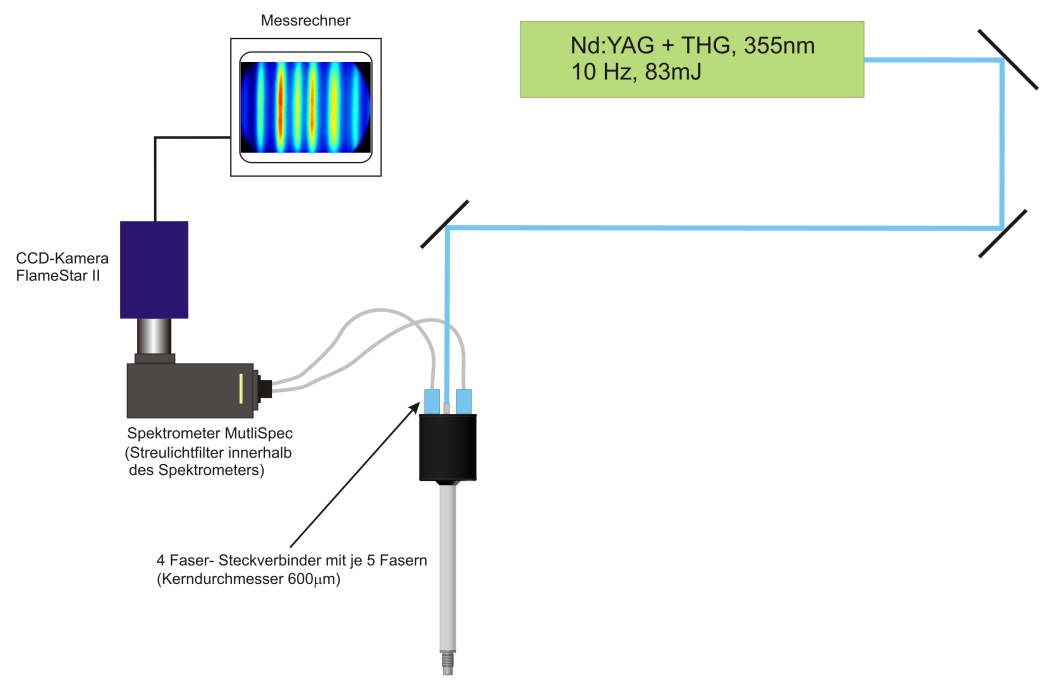

Abbildung 6.17: Schematischer Aufbau mit Freistrahleinkopplung des Lasers.
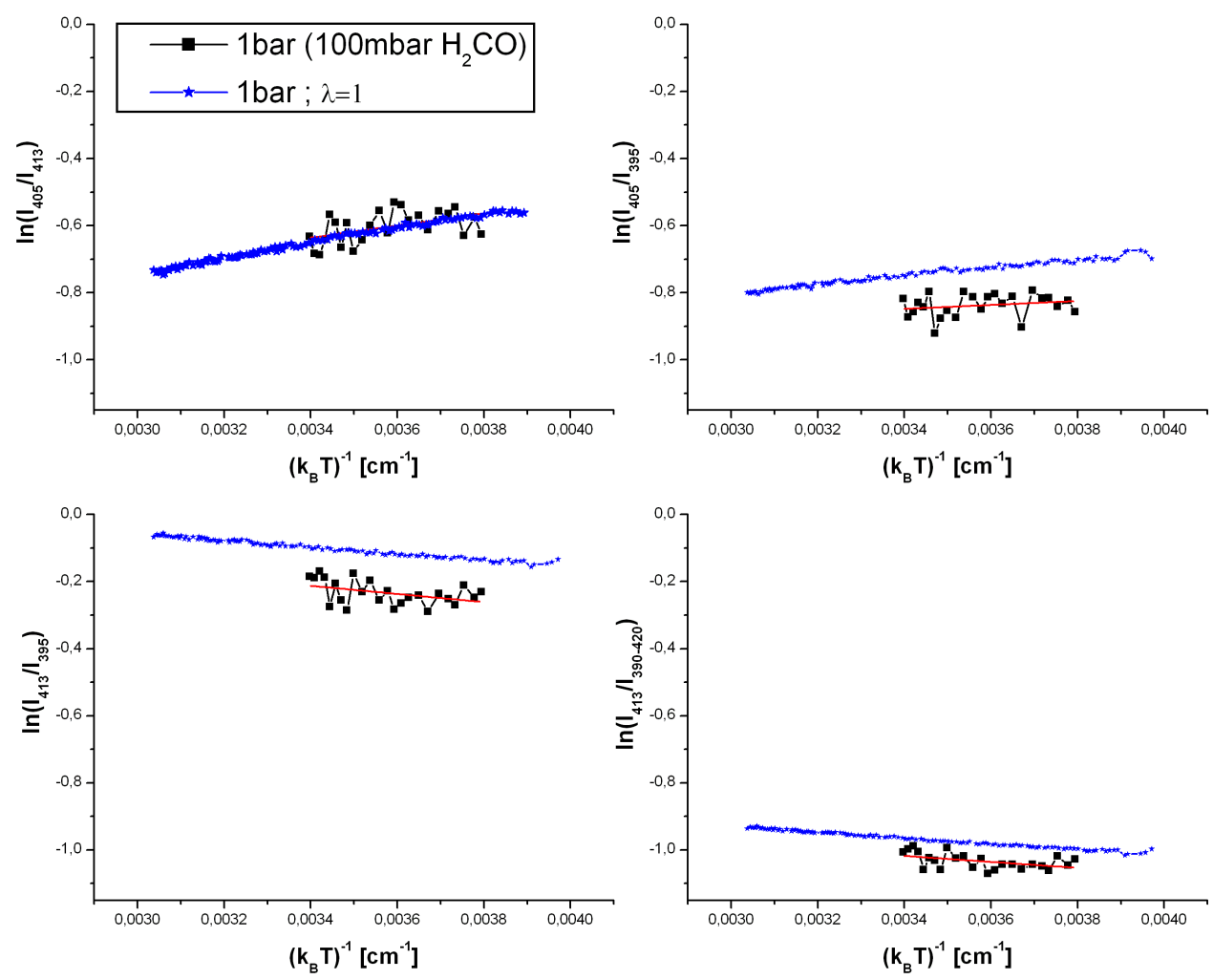

Abbildung 6.18: Durch die Messdaten resultierende Arrheniusauftragung, die mit dem Sensor der 1. Generation gemessen wurden. Zum Vergleich wurden die Daten der Kameramessungen ebenfalls dargestellt (blaue Kurven). 
Die starke Streuung der einzelnen Messwerte ist auf die geringe Detektion des Fluoreszenzsignals zurückzuführen. Hier spiegelt sich die Tatsache wider, dass eine ausreichende Umlenkung des Anregungslichtes nicht gegeben war und nur die Fasern nahe der angewinkelten Spiegelfläche zur Detektion beitrugen. Obwohl hier ein geringes Signal-zu-Rausch-Verhältnis vorliegt, existiert eine gute Übereinstimmung der Regressionsgeraden der sensorerfassten Werte mit den zuvor erbrachten Messungen für das Verhältnis 404nm/413nm. Somit besteht die Möglichkeit, mit den statischen Messungen an der Druckkammer Kalibrationen für dieses Verhältnis zu generieren.

\section{Messungen mit dem Sensorsystem der 2. Generation}

Der Versuchsaufbau des zweiten Sensorsystems entspricht dem Aufbau, wie er auch für die Motormessungen verwendet wird. Es findet hier der Einsatz des Strahlhomogenisators statt und es wurde das Kamerasystem mit der PTU9 verwendet. Abbildung 6.19 verdeutlicht den abgewandelten Aufbau. Um Zerstörungen der eingesetzten Optiken zu vermeiden, wurde die Laserenergie auf 60mJ reduziert.

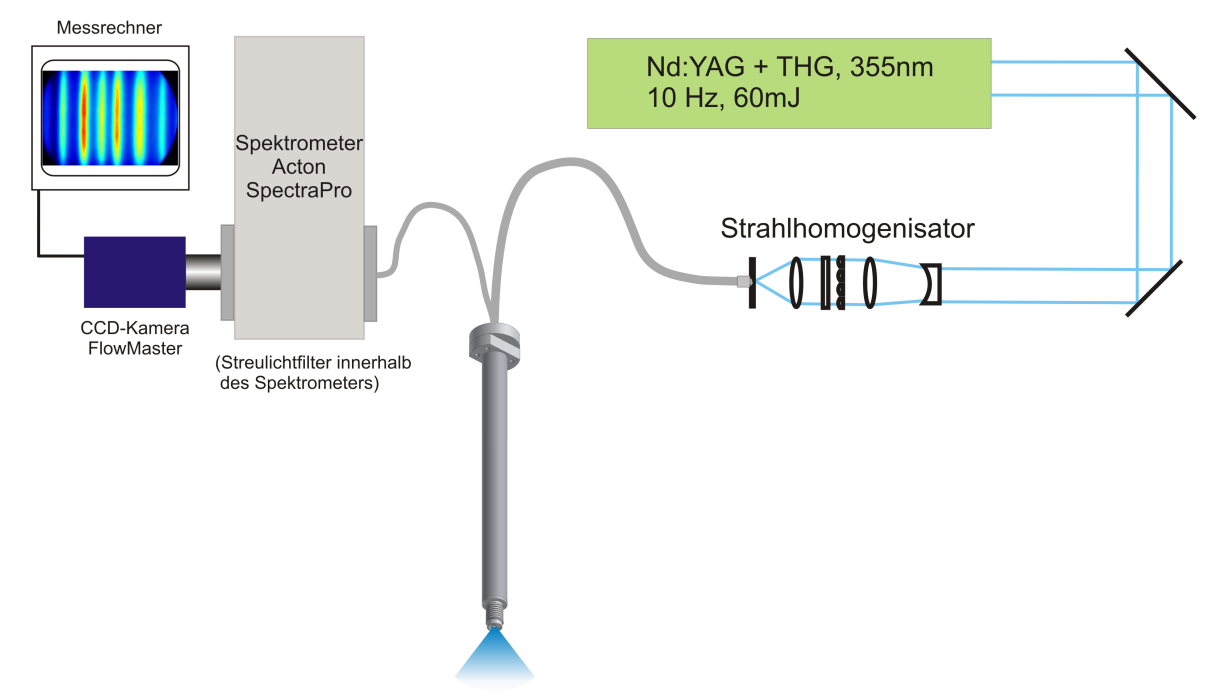

Abbildung 6.19: Schematischer Aufbau mit Strahlhomogenisator zur
Lasereinkopplung.

Die Detektionseinheit besteht bei diesen Messungen aus der FlowMaster Kamera und dem Spektrometer von Acton. Zusätzlich wurde mit Hilfe des Engine Simulators ein Triggerschema generiert, welches ein Motor bei einer Drehzahl von 1000min ${ }^{-1}$ erzeugt. Die Messungen wurden hier auf den Druckbereich von 1 bis 3bar erweitert. Der Temperaturbereich betrug wiederum $100^{\circ} \mathrm{C}$ bis $150^{\circ} \mathrm{C}$. Die Resultate ergaben ein analoges Gesamtbild wie in den vorangegangenen Messungen. In der Abbildung 6.20 sind diese vergleichend mit reinen Kameramessungen aufgetragen. 

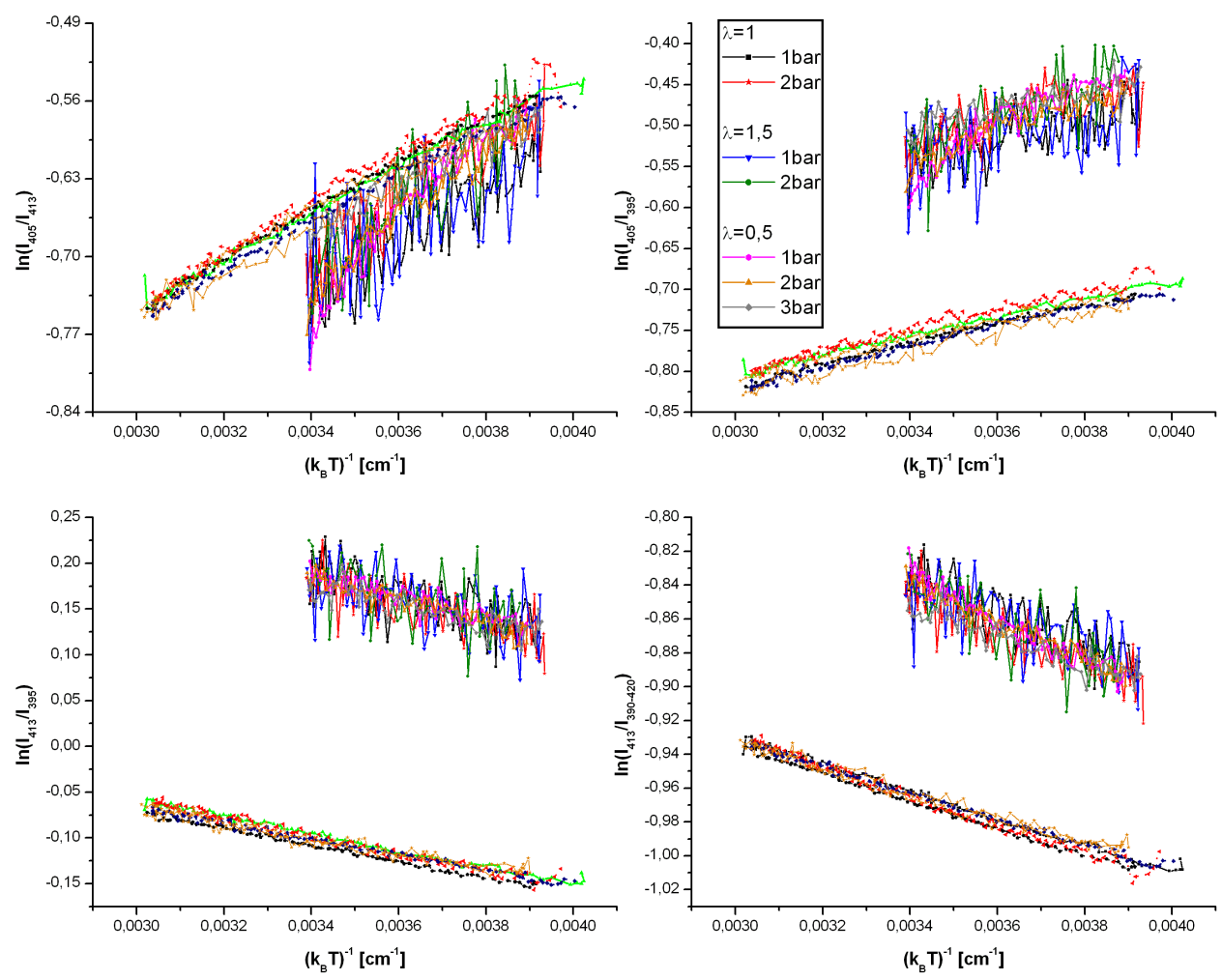

Abbildung 6.20: Arrheniusauftragung der mit dem Sensor 2 erfassten Daten. Auch hier wurden zum Vergleich die entsprechenden Daten der Kameramessungen aufgezeichnet. Diese sind durch den größeren Temperaturbereich erkennbar. 
Die sensorerfassten Messwerte weisen ebenfalls eine stärkere Streuung auf, als die Referenzwerte der statischen Messungen. Hier ist allerdings zu beachten, dass die Lichtleitung sowohl der Anregung als auch der Fluoreszenzdetektion komplett durch optische Fasern realisiert wird. Dadurch entstehen Lichtverluste, die aber durch on-chip-Integration oder Summation von Mehrfachaufnahmen kompensiert werden können. Des Weiteren ist der Einsatz von optischen Fasern die optimale Lösung für eine sehr dynamische Applikationsumgebung, wie sie beim Motor vorliegt. Die schon mit dem ersten Sensor erbrachte Erkenntnis über den stark approximativen Verlauf der Arrheniusauftragungen des Verhältnises 405nm/413nm konnte in diesen Testmessungen bestätigt werden. Somit erhärtet sich die Aussage, dass das Verfahren durch analytische Anwendung des Linienverhältnisses 405nm/413nm eine erhöhte Temperatursensitivität erhält, bei relativ guten Kalibrationsbedingungen, was sich wiederum auf die Genauigkeit auswirkt.

\subsection{Sensormessungen am Motor}

In diesem Abschnitt werden die Messungen vorgestellt, die unter motorischen Bedingungen den Einsatz des entwickelten Sensorsystems bewerten sollen. Neben der Darstellung aller relevanten Kenndaten des Motors wird auf die Vergleichbarkeit der chemischen und physikalischen Bedingungen innerhalb der Druckkammer und des Motors eingegangen. Die Bestimmung von Temperaturwerten innerhalb des Brennraums und eine abschließende Betrachtung der erfassten Resultate runden diesen Abschnitt ab.

\subsubsection{Physikalische Größen des Motors}

Die applikationsnahen Messungen fanden am institutseigenen Motorprüfstand statt. Dieses System besteht im wesentlichen aus einer sogenannten Pendelmaschine und dem Motor selbst. Mit Hilfe der Pendelmaschine kann der Motor ungezündet, im sogenannten geschleppten Zustand, betrieben werden, indem über die Antriebswelle der Motor angetrieben werden kann. Im gezündeten Zustand wird wiederum die durch den Motor erzeugte mechanische Energie von der Pendelmaschine aufgenommen. Da es sich bei dem entwickelten Sensor um einen nicht zündfähigen Sensor handelt, welcher über die Zündkerzenbohrung in den Brennraum gelangt, finden hier nur Untersuchungen im ungezündeten Betrieb statt. Der eingesetzte Motor von der Firma Volkswagen ist ein Vier-Zylinder Einspritzmotor mit Zwei-Ventil-Technologie. Die für diese Arbeit relevanten Kenndaten des Motors sind in Tabelle 6.2 aufgeführt. Um stöchiometrische Berechnungen im Motor durchführen zu können, muss zum einen das kurbelwinkelabhängige Zylindervolumen $V(\varphi)$ ( $\varphi$ entspricht dem Kurbelwinkel) bestimmt werden. Dies kann mit der Gleichung 


\begin{tabular}{|c|c|}
\hline Bohrungsdurchmesser $d$ & $82,5 \mathrm{~mm}$ \\
Hub $s$ & $92,8 \mathrm{~mm}$ \\
Pleuellänge $l$ & $159 \mathrm{~mm}$ \\
Verdichtung $\epsilon$ & 10,0 \\
Hubvolumen $V_{h}$ & $0,496 \mathrm{~L}$ \\
\hline
\end{tabular}

Tabelle 6.2: Kenngrößen des verwendeten Motors.

$$
V(\varphi)=V_{h} \cdot\left(\frac{1}{\epsilon-1}+\frac{1-\cos \varphi}{2}+\frac{1}{2 l_{s}}\left(1-\sqrt{1-l_{s}^{2} \cdot \sin ^{2} \varphi}\right)\right)
$$

realisiert werden. Hierbei ist $l_{s}$ das sogenannte Schubstangenverhältnis (Bohrungsradius/Pleuellänge).

Des Weiteren ist der im Brennraum vorhandene Druck von essenzieller Bedeutung. Dieser wird mit Hilfe piezoelektrischer Drucksensoren erfasst. Da mit diesem Prinzip nur Druckdifferenzen messbar sind, gibt es keine Aussage über die Absolutwerte des Drucks. Um Absolutwerte zu erhalten, wird eine thermodynamische Nulllinienfindung nach Hohenberg durchgeführt. Dieses Verfahren basiert auf dem Vergleich der Kompressionslinie mit einer theoretischen Polytropen. Für eine polytrope Zustandsänderung gilt

$$
\frac{p_{2}}{p_{1}}=\left(\frac{V_{1}}{V_{2}}\right)^{n}
$$

mit $n$ als Polytropenexponent. Für Ottomotoren liegt der Polytropenexponent zwischen 1,33 und 1,35. Bei Dieselmotoren hingegen zwischen 1,36 und 1,38. Wird die Druckdifferenz zweier Messwerte $\Delta p=p_{2}-p_{1}$ in Gleichung 6.19 eingesetzt, ergibt sich für den Absolutdruck

$$
p_{1}=\frac{\Delta p}{\left[\left(\frac{V_{1}}{V_{2}}\right)^{n}-1\right]} .
$$

Nähere Information zu diesem bewährten und sehr genauen Messprinzip sind in [57] enthalten. Abbildung 6.21 zeigt eine gemessene kurbelwinkelaufgelöste Druckkurve für einen kompletten Motorzyklus bei einer Drehzahl von $1000 \mathrm{~min}^{-1}$, wie er auch bei den Messungen vorlag. 


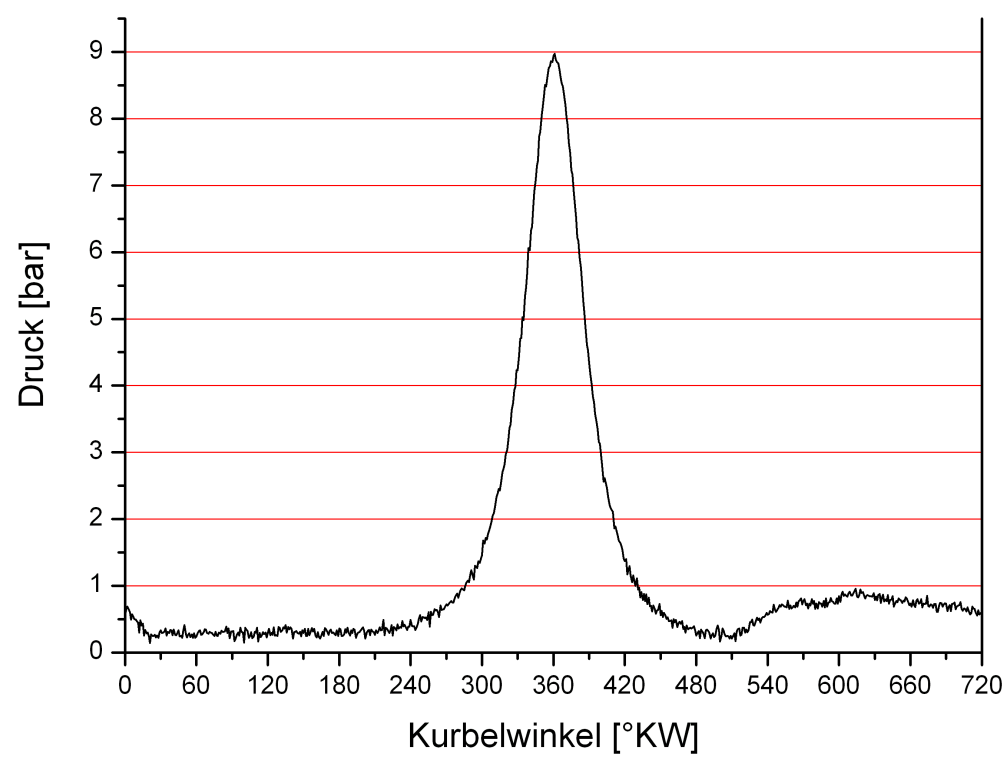

Abbildung 6.21: Kurbelwinkelaufgelöste Druckkurve für einen Motorzyklus bei einer Drehzahl von $1000 \mathrm{~min}^{-1}$. Die Absolutdruckwerte sind mit der Nullpunktfindung korregiert worden.

\subsubsection{Stöchiometrie der Gemische}

Nachdem die nötigen motorischen Größen ermittelt wurden, soll nun überprüft werden, wie genau die in der Druckkammer generierten $\lambda$-Werte mit den stöchiometrischen Bedingungen im Motor übereinstimmen. Dazu werden die Stoffmengen des Kraftstoffs für beide Umgebungsbedingungen bestimmt und verglichen. Zuerst soll die Stoffmenge des Kraftstoffs im Motor berechnet werden. Ausgang dieser Betrachtung ist der motorische Zyklusabschnitt, bei dem sich die Ventile schließen, nachdem eingesaugt wurde. Laut den Kenndaten aus dem Werkstattheft des Motors befindet sich dieser Zustand bei einem Kurbelwinkel von 224 . Mit Hilfe der in Kapitel 6.5.1 vorgestellten Berechnungen erhält man für diesen Kurbelwinkel folgende Werte für den Druck, die Temperatur und das Volumen:

$$
\begin{aligned}
p & =1,76 \mathrm{bar} \\
T & =313,02 \mathrm{~K} \\
V & =0,499 \mathrm{~L}
\end{aligned}
$$


Mit Hilfe des idealen Gasgesetzes kann die Stoffmenge des Luft-Kraftstoff-Gemisches $n_{a / f}$ ermittelt werden.

$$
n_{a / f}=\frac{p \cdot V}{R \cdot T}=\frac{1,76 \mathrm{bar} \cdot 0,499 \mathrm{~L} \cdot \mathrm{mol} \cdot \mathrm{K}}{0,0831472 \cdot \mathrm{bar} \cdot \mathrm{L} \cdot 313,02 \cdot \mathrm{K}}=33,74 \mathrm{mmol}
$$

Unter der Annahme, dass im Motorenbetrieb ein $\lambda$-Wert von 1 im Brennraum generiert wird, kann nach [58] die Stoffmenge des Kraftstoffs mit

$$
n_{\text {Kraftstoff }}=\frac{n_{\text {Luft }} \cdot M_{\text {Luft }}}{\lambda \cdot 14,7 \cdot M_{\text {Kraftstoff }}}
$$

ermittelt werden. Die molare Masse der Luft beträgt $M_{\text {Luft }}=28,97 \mathrm{~g} / \mathrm{mol}$. Mit Hilfe der stöchiometrischen Angaben aus den Gleichungen 6.1 bis 6.3 ergibt sich für die molare Masse des Kraftstoffes der Wert $M_{\text {Kraftstof }}=189,96 \mathrm{~g} / \mathrm{mol}$. Die Stoffmenge der Luft kann durch

$$
n_{\text {Luft }}=n_{a / f}-n_{\text {Kraftstoff }}
$$

in Gleichung 6.22 ersetzt werden. Mit obiger Annahme folgt $\lambda=1$ und es ergibt sich für die Stoffmenge des Kraftstoffes im Motor

$$
n_{\text {Kraftstoff }}=\frac{n_{a / f}}{14,7 \cdot \frac{M_{\text {Kraftstoff }}}{M_{\text {Luft }}}+1}=\frac{0,03374 \mathrm{~mol}}{14,7 \cdot \frac{189,96 \mathrm{~g} \cdot \mathrm{mol}}{28,97 \mathrm{~g} \cdot \mathrm{mol}}}=0,346 \mathrm{mmol}
$$

Innerhalb der Druckkammer wurden die $\lambda$-Werte über den Druck eingestellt. Mit Hilfe der Gleichung 6.14 werden die entsprechenden Kraftstoffdrücke berechnet. Mit Hilfe dieser Werte wurde im Versuch die entsprechende Menge des Gemisches über ein Septum in die Druckkammer injiziiert.

Der obige motorische Zustand entspricht den Druckkammer-Messreihen für $\lambda=1$ mit einem Gesamtdruck von 2bar. Diese Bedingung soll zum Vergleich herangezogen werden. Für den Kraftstoffdruck $p_{\text {Gemisch }}$, der zum Zeitpunkt der Befüllung vorherrschenden Temperatur $T_{\text {Kammer }}$ und dem Volumen der Druckkammer $V_{\text {Kammer }}$ ergeben sich folgende Werte

$$
\begin{aligned}
p_{\text {Gemisch }} & =96 \mathrm{mbar} \quad \text { (bei Gesamtdruck } 2 \text { bar) } \\
T_{\text {Kammer }} & =363 \mathrm{~K} \\
V_{\text {Kammer }} & =0,933 \mathrm{~L}
\end{aligned}
$$

Für die Stoffmenge des Kraftstoffs ergibt sich über das ideale Gasgesetz ein Wert von

$$
n_{\text {Kraftstoff }}=\frac{p \cdot V}{R \cdot T}=\frac{0,096 \mathrm{bar} \cdot 0,933 \mathrm{~L} \cdot \mathrm{mol} \cdot \mathrm{K}}{0,0831472 \cdot \mathrm{bar} \cdot \mathrm{L} \cdot 363 \cdot \mathrm{K}}=0,3 \mathrm{mmol} .
$$

Die gute Übereinstimmung der beiden Werte aus Gleichung 6.24 und 6.25 zeigen, dass die stöchiometrischen Bedingungen in der Druckkammer den motorischen Bedingungen entsprechen. 


\subsubsection{Temperaturbestimmung nach Arrhenius}

Da die Resultate der statischen Messungen unter motorischen Bedingungen recht gut den stöchiometrischen Verhältnissen innerhalb des Brennraums entsprechen, können diese zur Kalibration des Messverfahrens herangezogen werden. Bei den Messungen am Motor wurde der für das Verfahren entscheidende Spektralbereich der Fluoreszenz von Formaldehyd detektiert und die Verhältnisbildung der in Kapitel 4.5.1 vorgestellten Linienpaare durchgeführt. Der daraus erfolgte Verhältniswert $F_{1} / F_{2}$ führt über die logarithmische Arrhenius-Funktion (siehe Gleichung 6.15) zur Temperaturinformation. Aufgelöst nach der Temperatur ergibt sich folgende Auswerteformel:

$$
T=\frac{\Delta E \cdot 1,98648}{\left(\ln \left(\frac{F_{1}}{F_{2}}\right)-A\right) \cdot k_{B}}
$$

Der Faktor 1,98648 ist ein Energie-Umrechnungfaktor von der Einheit J auf die Einheit $\mathrm{cm}^{-1}$. Innerhalb der logarithmischen Arrhenius-Auftragung entspricht $\Delta E$ der Steigung und der Faktor $A$ dem Ordinatenabschnitt. Physikalisch entspricht $\Delta E$ der Energiedifferenz der beiden betrachteten Energieniveaus.

Leider stellte sich während der Motormessungen heraus, dass die Fluoreszenz von Formaldehyd nur am stehenden Motor detektiert werden konnte. Im folgenden Kapitel soll näher auf diese Problematik eingegangen werden. Aufgrund dieser Tatsachen wurde mit Hilfe der Antriebswelle ein Kurbelwinkel eingestellt, bei dem alle Ventile geschlossen sind und der Kolben sich nahe des unteren Totpunkts (UT) befand. Da eine Detektion unter diesen Bedingungen recht schwierig ist, wurde eine mit Formaldehyd angereichte Ethanollösung (30 Massen-\%) direkt in den Brennraum eingespritzt. Nach Einbau des Sensors wurde der Motorblock mit Hilfe des Wasserkreislaufes auf eine hohe Betriebstemperatur gebracht. Bei den Messungen betrug die Temperatur des Wasserkreislaufs $71,1^{\circ} \mathrm{C}$, welche auch die technisch maximal realisierbare Temperatur darstellt. Die Messungen wurden mit Hilfe der FlowMasterKamera und des Acton-Spektrometers durchgeführt. Dabei wurden Mehrfachaufnahmen generiert (Mittelung über 50 Bilder) und zu einem Spektrum integriert. Nach Anwendung des Auswerte-Algorithmus wurde die Temperatur mit Hilfe der in den statischen Messungen erbrachten Kalibrationsfaktoren $\Delta E$ und $A$ errechnet. Um eine breite Bewertung des Verfahrens in Bezug auf Querempfindlichkeiten zu erhalten, wurden die Kalibrationsfaktoren aller motorrelevanter Messungen (gemittelte Werte) herangezogen und verglichen. Es stellte sich heraus, dass mit dem Linienverhältnis $405 \mathrm{~nm} / 413 \mathrm{~nm}$ die besten und stabilsten Werte erhalten wurden. In Tabelle 6.3 sind die mit diesem Linienpaar erfassten Daten dargestellt.

Ein Problem stellt der Referenzwert von $71,1^{\circ} \mathrm{C}$ dar. Dieser ist nur bedingt aussagekräftig, da dies der Temperatur des Wassers entspricht, welches den Motorblock umspült. Aufgrund von Wärmeverlusten durch den Motorblock wird die tatsächliche Temperatur innerhalb des Brennraums geringer sein. Dies kann geringfügig durch 


\begin{tabular}{|c|c|l|c|}
\hline $\begin{array}{c}\lambda \text {-Wert } \\
\text { der statischen Messungen }\end{array}$ & $\begin{array}{c}\Delta E \\
{\left[\mathrm{~cm}^{-1}\right]}\end{array}$ & $A$ & $\begin{array}{c}\text { Temperatur } \\
{\left[{ }^{\circ} \mathrm{C}\right]}\end{array}$ \\
\hline 0,5 & 224,527 & $-1,437$ & 67,7 \\
1,0 & 225,648 & $-1,44$ & 68,4 \\
1,5 & 216,804 & $-1,413$ & 64,3 \\
alle $\lambda$ gemittelt & 222,326 & $-1,43$ & 68,8 \\
alle $\lambda$, Sensor 2 & 283,961 & $-1,686$ & 68,2 \\
\hline
\end{tabular}

Tabelle 6.3: Kalibrationsfaktoren der motorrelevanten statischen Messungen und die damit bestimmten Temperaturwerte für den Brennraum. Die Werte wurden mit dem Linienverhältnis 405nm/413nm ermittelt.

eine lange Aufheizdauer kompensiert werden. Allerdings ist dennoch eine Abschätzung möglich, ob das Verfahren Temperaturwerte liefert, die in einem realistischen Rahmen liegen. Da innerhalb des Brennraums nur eine maximale Temperatur von $71,1^{\circ} \mathrm{C}$ vorliegen kann, befinden die mit dem Verhältnis $405 \mathrm{~nm} / 413 \mathrm{~nm}$ berechneten Werte in guter Näherung zum tatsächlichen Temperaturwert.

\subsubsection{Abschließende Betrachtung}

Die Untersuchungen bei laufendem Motor ergaben, dass das Sensorsystem einwandfrei arbeitet. Durch kontinuierliche Aufnahmen konnte der Einspritzvorgang spektral beobachtet werden, indem ein Fluoreszenzsignal kurz nach dem Einschalten der Einspritzpumpe detektiert werden konnte, welches langsam wieder verschwand, nachdem die Einspritzung eingestellt wurde. Die prinzipielle Funktion des Sensors konnte somit nachgewiesen werden. Ein Problem stellte aber das detektierte, starke und breitbandige Fluoreszenzsignal dar, welches nicht vom Formaldehyd stammt. Da zuvor an dem Motor Versuche mit Realkraftstoff durchgeführt wurden, war der Motor stark kontaminiert mit aromatischen Kohlenwasserstoffen, die Bestandteile des Kraftstoffs sind. Diese liefern ein starkes Fluoreszenzsignal, welches sich über den betrachteten Spektralbereich überlagert. Somit wurde eine gründliche Reinigung der Einspritzanlage und sogar der Austausch einzelner Komponeten vorgenommen, um diesen Störeinfluss zu eliminieren. Während die Kraftstoffversorgung recht gut zu reinigen ist, stößt man innerhalb des Brennraums an die Grenzen. Hier stellte sich heraus, dass auf dem Kolben befindliche Rückstände und Rußpartikel ebenfalls eine starke Fluoreszenz erzeugen. Auch durch einen längeren gezündeten Motorenbetrieb konnte eine Reinigung durch Verbrennung nicht erzielt werden. Erst eine Analyse einzelner Kurbelwinkel bei stehendem Motor und manueller Einspritzung eines Gemmisches machte Formaldehyd nachweisbar. Es war zu beobachten, dass die Kolbenfläche im OT ein starkes Signal generierte, welches verschwand, je weiter sich der Kolben nach unten bewegte. Nachdem der Kolben so weit vom Sensor entfernt 
war, dass das breitbandige Signal verschwand, wurde ein schwaches Spektrum von Formaldehyd detektiert.

Somit existiert zum einen das Problem, dass in dieser Konfiguration eine Temperaturmessung innerhalb der Kompression nicht möglich ist, aufgrund der von der Kolbenstellung abhängigen, überlagerten Fremdfluoreszenz. Dies bedingt wiederum ein weiteres Problem. Um das im Gemisch enthaltene Formaldehyd in die Gasphase zu bekommen, benötigt man Temperaturen, die nur in der kompressiven Phase des Motors entstehen. Um abzuschätzen, welcher Temperaturbereich erreicht werden muss, wurde mit Hilfe der Druckkammer die Siedetemperatur der Ethanol/Formaldehyd-Lösung untersucht. Um die gleichen Bedingungen zu haben, wie sie im Motor vorliegen, wurde das Gemisch unter Atmosphärendruck injiziiert und in der abgeschlossenen Kammer erwärmt. Dabei stellte sich heraus, dass die Lösung bei einer Temperatur von ungefähr $170^{\circ} \mathrm{C}$ siedet. Dieser Versuch unterscheidet sich von den statischen Messungen in der Druckkammer dadurch, dass die Gemische der statischen Messreihen in eine evakuierte Kammer eingespritzt wurden und somit andere Anfangsbedingungen herrschten.

Unter der Annahme einer adiabatischen Zustandsänderung im Motor kann durch die Zustandsgleichung

$$
T_{Z y l}=\left(\frac{p_{Z y l}}{p_{e i n}}\right)^{\frac{\kappa-1}{\kappa}} \cdot T_{e i n}
$$

die kurbelwinkelaufgelöste Temperatur bestimmt werden. Die Indizes $Z y l$ und ein beziehen sich auf die physikalischen Größen Druck $p$ und Temperatur $T$ innerhalb des Zylinders und am Einlassort. $\kappa$ ist der sogenannte Isentropenexponent und hat für das angesaugte Gasgemisch den Wert 1,32. Abbildung 6.22 zeigt das Resultat für den verwendeten Motor. 


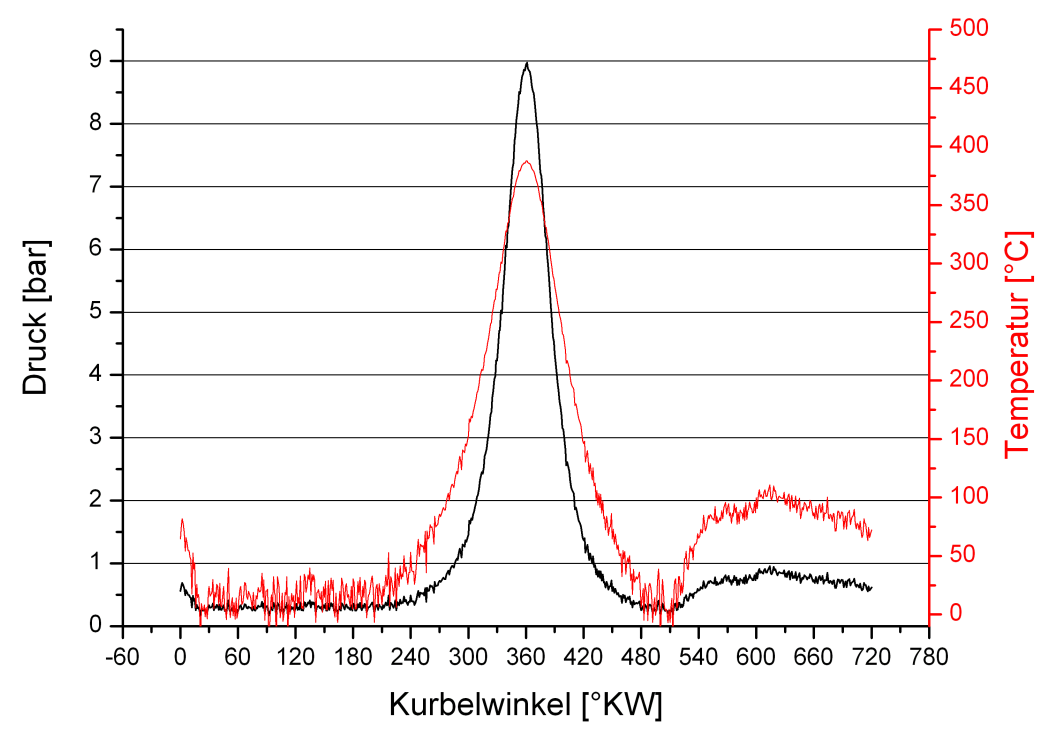

Abbildung 6.22: Kurbelwinkelaufgelöste Druck- und Temperaturkurve für einen Motorzyklus.

In der Abbildung ist zu sehen, dass die benötigte Temperatur ab einem Kurbelwinkel von ungefähr $300^{\circ} \mathrm{KW}$ vorliegt. In dieser Stellung wirkt sich allerdings schon das Störsignal durch den Kolben aus.

Aufgrund dieser Tatsache ist die Detektion der Fluoreszenz von Formaldehyd recht schwierig, da der stehende Motor nicht auf die erforderliche Temperatur erwärmt werden kann. Insofern ist das in diesem Zustand detektierte Fluoreszenzsignal sehr schwach. Mit Hilfe von Mehrfachaufnahmen wurde dieses Signal verstärkt. Dadurch konnte eine Analyse realisiert werden, die ein erstaunliches Resultat hervorbrachte. Wie in Tabelle $6.3 \mathrm{zu}$ sehen ist, erbrachte die Temperaturbestimmung unter Verwendung des Lininenpaares $405 \mathrm{~nm} / 413 \mathrm{~nm}$ sehr realistische Temperaturwerte. Dies zeigt, dass das Verfahren zwar noch einiger Verbesserung hinsichtlich der Sensorkonstruktion bedarf. Die physikalischen Grundlagen, auf die das Messverfahren basiert, können jedoch zur Temperaturbestimmung in der Motordiagnostik herangezogen werden. 


\section{Kapitel 7}

\section{Zusammenfassung}

In dieser Arbeit wurde der Frage nachgegangen, wie die Temperatur eines KraftstoffLuft-Gemisches innerhalb des Brennraums erfasst werden kann. Hierbei bildet die laserunterstützte optische Messtechnik die Basis zur Erfassung der Temperaturinformation, da sie durch ihre berührungslose Anwendung den ottomotoriscchen Brennverlauf am geringsten beeinflusst. Mit Hilfe der Laserinduzierten Fluoreszenz und des Einsatzes eines Tracermoleküls kann über die Zwei-Linien-Thermometrie die Gastemperatur extrahiert werden. Das Tracermolekül wird durch Laserstrahlung zur Emission mehrerer Fluoreszenzlinien angeregt. Die Besetzungen der einzelnen Energieniveaus, die für die spektralen Emissionslinien verantwortlich sind, sind durch eine Boltzmann-Verteilung beschreibbar und von der Temperatur des Gases abhängig. Eine Verhältnisbildung von zwei Emissionslinien gibt demnach Auskunft über die momentane Temperatur. Analytisch besteht dieses Linienverhältnis mit der thermodynaischen Größe über die Arrhenius-Funktion in direkter Relation. Eine Grundvoraussetzung für die Anwendung dieses Verfahrens ist, dass die sogenannte Thermalisierung innerhalb der Fluoreszenzlebensdauer abgeschlossen sein muss. Unter der Thermalisierung ist der Prozess zu verstehen, der die Verteilung der Energie auf die einzelnen angeregten Energieniveaus beinhaltet. Nur die Momentaufnahme des Systems bei abgeschlossener Umverteilung gibt eine Auskunft über die Temperatur. Somit war eines der ersten Ziele dieser Arbeit, das Tracermolekül Formaldehyd daraufhin zu prüfen, ob seine spektroskopischen Eigenschaften für das eingesetzte Messverfahren geeignet sind. Die hier vorgestellten spektroskopischen Untersuchungen zeigten Resultate, welche die Einsatzfähigkeit dieser Substanz untermauern. Druckabhänige Fluoreszenzlebensdauermessungen zeigten eine Lebensdauer der Fluoreszenz von 33 bis 5,9 ns. Die Thermalisierungszeit von Formaldehyd hingegen beläuft sich zwischen 120 und 7,5 ps. Statische Untersuchungen an einer Druckkammer unter motorrelevanten Bedingungen offenbarten drei für das Verfahren geeignete Linienpaare. Dabei handelte es sich um die Verhältnisbildungen aus den Übergängen $2_{1}^{0} 4_{1}^{0}(395 \mathrm{~nm}), 4_{3}^{0}(405 \mathrm{~nm})$ und $2_{1}^{0} 4_{2}^{1}(413 \mathrm{~nm})$. Besonders das Linienpaar 405nm/413nm zeichnete sich durch sehr geringe Querempfindlichkeiten bezüglich Druck- und Konzentrationsschwankungen aus. Des Weiteren stellte sich in Versuchen heraus, dass ein mit Formaldehyd angereichertes Gemisch recht einfach 
durch Lösen von Paraformaldehyd, einer polymerisierten Form von Formaldehyd, in Ethanol erzeugt werden kann. Diese Lösung lässt sich ebenfalls problemlos mit dem Testkraftstoff Isooktan vermischen, welcher wiederum keinen Einfluss auf die Fluoreszenzeigenschaften des Formaldehyds hat. Durch stöchiometrische Berechnungen wurden die $\lambda$-Werte berechnet und mit den stöchiometrischen Verhältnissen im Brennraum verglichen und angepasst. Somit konnten identische Bedingungen in der Druckkammer und im Motor gewährleistet werden.

Die Möglichkeit, dieses Molekül nur mit Hilfe eines frequenzverdreifachten Nd:YAGLasers anregen zu können, vereinfachen das Verfahren deutlich, da der optische Aufbau dadurch weniger Aufwand bedarf. Hier liegt auch der Unterschied zu herkömmlichen Verfahren, bei denen die Abfrage zweier Besetzungen im Grundzustand durch zwei Lasersysteme realisiert wird. Die Anpassung des Messverfahrens auf die motorische Applikation erfolgte durch die Entwicklung zweier Sensorsysteme. Dabei wird das Sensorsystem über die Zündkerzenbohrung in den Brennraum integriert. Der Motor kann dadurch im ungezündeten Betrieb untersucht werden. Somit ist die gesamte in den Brennraum hineinragende Fläche zur Fluoreszenzdetektion verwendbar, da kein Raum durch eine Zündanlage eingenommen wird. Weiterhin entfällt ein durch den Zündfunken entstehendes Hintergrundsignal, welches die Detektion des Fluoreszenzsignals erheblich stören würde. Die Untersuchungen im ungezündeten Betrieb sind hier ausreichend, da die Temperaturentwicklung des Gemisches bis zur Zündung von Interesse ist, um den Einfluss des thermischen Zustands des Gemisches auf die Verbrennung zu evaluieren.

Die Erarbeitung und Konstruktion des ersten Prototyps fand unter Zusammenarbeit mit der Firma LaVision statt. Erste motorrelevante Testmessungen an der Druckkammer lieferten die ersten Ergebnisse, die eine Temperaturmessung zuließen. Eine gute Reproduzierbarkeit wurde erneut mit dem Linienverhältnis 405nm/413nm erzielt. Dieser Sensor hatte allerdings eine geringe Einsatzdauer, da durch eine Fokusbildung der Laserstrahlung eine Beschädigung der reflektiven Oberfläche entstand. Die mit dem ersten Sensorsystem erbrachten Erkenntisse wurden daraufhin genutzt, um einen zweiten Sensor zu entwickeln. Aufgrund verbesserter optischer Führung der gesamten Lichtleitung konnte ein stabiler Einsatz des zweiten Prototyps ermöglicht werden. Auch bei diesem Sensorkonzept konnte eine gute Reproduzierbarkeit mit Hilfe der Linien $405 \mathrm{~nm}$ und $413 \mathrm{~nm}$ erbracht werden.

Den finalen Schritt dieser Arbeit bildet der Einsatz des entwickelten Sensorsystems am institutseigenen Motorprüfstand. Es wurden Experimente unter motorisch realen Bedingungen durchgeführt und erstmals Erfahrungen mit diesem Temperaturmessverfahren gesammelt. Dabei integrierte sich das Sensorkonzept ohne negativen Einfluss auf den laufenden Motorenbetrieb. Die Messungen ergaben jedoch, dass die Kontamination des Brennraums und der Einspritzanlage durch Verbrennungs- und Kraftstoffrückstände ein zu starkes Hintergrundsignal lieferte, welches die Detektion von Formaldehyd unterbunden hatte. Eine Reinigung der Einspritzanlage war zwar erfolgreich, doch eine vollständige Befreiung des Brennraums von den Kon- 
taminanten erwies sich als nicht durchführbar. Die Sensorkonstruktion war darauf ausgelegt, dass eine direkte Laserbestrahlung in den Brennraum zur Anregung des Formaldehyds führen sollte. Die Temperatur, die nötig ist, um das in Lösung vorliegende Formaldehyd in die Gasphase zu bekommen, wird erst in der kompressiven Phase des Motorzyklus erreicht. In diesem Zustand ist der Kolben allerdings schon so nahe am Sensor, dass die auf der Kolbenoberfläche befindlichen Rückstände das zuvor beschriebene Störsignal erzeugen. Die an der Druckkammer durchgeführten Messungen zur Siedetemperatur des mit Formaldehyd angereicherten Ethanols unter motorischen Bedingungen ergab eine Temperatur von ungefähr $170^{\circ} \mathrm{C}$.

Trotz dieser Tatsachen war eine Evaluation des Messverfahrens möglich. Es wurde eine Formaldehyd/Ethanol-Lösung manuell in den Brennraum injiziiert und der abgeschlossene Brennraum mit integriertem Sensor über den Wasserkreislauf erwärmt. Ausgangspunkt der Messungen am stehenden Motor war der Motorzyklus bei $360^{\circ} \mathrm{KW}$. In diesem Zustand befindet sich der Kolben am oberen Totpunkt und generierte die erwartete Fremdfluoreszenz. Es wurde sukzessiv der Kolben in umgekehrter Richtung ,nach unten“" gedreht, um ein Öffnen der Ventile zu vermeiden und weiterhin ein abgeschlossenes Volumen zu haben. Mit zunehmendem Abstand zwischen Kolben und Sensor verringerte sich die Fremdfluoreszenz und bei völliger Abwesenheit des Störsignales konnten die drei Emissionslinien des Formaldehyds detektiert werden. Da das Fluoreszenzsignal sehr schwach war, wurde dieses durch Integration von Mehrfachaufnahmen (50 Bilder) verstärkt. Mit Hilfe dieser spektralen Information war es möglich, mit den Messverfahren einen realistischen Temperaturwert zu erhalten. Um den Motor erwärmen zu können, wurde der Wasserkreislauf verwendet, der den Motorblock umspült. Die dabei technisch maximal erreichbare Temperatur betrug $71,1^{\circ} \mathrm{C}$, was unterhalb der gemessenen Siedetemperatur der Formaldehyd/Ethanol-Lösung liegt. Daher konnte das Gemisch, welches manuell in den Brennraum injiziiert wurde, nur zu einem sehr geringen Teil in die Gasphase gebracht werden. Trotz dieser schwierigen Bedingungen war es möglich, mit Hilfe des Linienverhältnisses $405 \mathrm{~nm} / 413 \mathrm{~nm}$ eine Temperatur von $68^{\circ} \mathrm{C}$ innerhalb des Brennraums zu messen. Der einzig mögliche Referenzwert ist die Temperatur des Wasserkreislaufs mit dem schon erwähnten Wert von $71,1^{\circ} \mathrm{C}$. Da innerhalb des Brennraums maximal dieser Temperaturwert vorliegen kann, ist eine realistische Einschätzung des berechneten Temperaturwertes als gerechtfertigt einzustufen. Dieses Ergebnis, welches weit unter Siedetemperatur erzielt wurde, zeigt, dass die Sensitivität des Messverfahrens hoch ist und dies innerhalb der motorischen Umgebung anwendbar ist. Die Messungen am Motor zeigten auf, dass die technische Umsetzung des Senorkonzeptes überarbeitet werden muss. Es zeigte aber auch, dass das in dieser Arbeit vorgestellte physikalische Messprinzip unter motorischen Bedingungen erfolgreich eingesetzt werden kann. 



\section{Kapitel 8}

\section{Ausblick}

Sowohl die statischen Messungen des Kamerasystems als auch diejenigen des Sensorsystems bestätigen, dass dieses LIF-basierte Messverfahren mit Formaldehyd als Tracermolekül in der Motordiagnostik durchaus erfolgreich eingesetzt werden kann. Die ersten Testmessungen am Motor lieferten Erkenntnisse, die auf konstruktive Veränderungen des Sensors hinweisen, der Anwendung des Messverfahrens aber nicht widersprechen. Ein nächster Schritt ist somit die konstruktive Optimierung des Sensorsystems. Eine verbesserte Konstruktion beinhaltet das Gestalten eines Messvolumens, welches von der Fremdfluoreszenz der Kontaminaten entkoppelt ist, so dass nur die Fluoreszenz des Tracermoleküls erfasst wird. Neben der recht guten Charakteriesierung des Emissionsspektrums von Formaldehyd birgt diese Substanz eine weitere für die motorische Applikation positive Eigenschaft. Innerhalb des Verbrennungsprozesses entsteht in einer Kettenverzweigung Formaldehyd als Zwischenprodukt. Eine Erweiterung dieses Verfahrens auf die Temperaturmessung der sogenannten Vorreaktionsphase, in der sich Formaldehyd bildet, kann aufgrund dieses Moleküls ermöglicht werden. Weiterhin ist der Einsatz des Verfahrens durch den leicht zu realisierenden Zusatz von Formaldehyd, so wie es auch in dieser Arbeit verwendet wurde, in anderen Motorzyklen durchführbar.

Unter der Verwendung eines Glasringmotors besteht sogar die Möglichkeit, dieses Verfahren um eine Dimension zu erweitern und mit Hilfe von zwei Kamerabildern, welche mit Hilfe entsprechender Spektralfilter für jeweils eine Wellenlänge sensibilisiert werden können, eine zweidimensionale Temperaturverteilung zu erhalten. 



\section{Anhang A}

\section{Technische Zeichnungen des Sensorsytems}

In diesem Anhang befinden sich die Konstruktionszeichnungen des FaserbündelSensorsystems, welches innerhalb dieser Arbeit entwickelt wurde.

\section{A.1 Konstruktionszeichungen des Sensors der 1. Generation}

A.2 Konstruktionszeichungen des Sensors der 2. Generation 


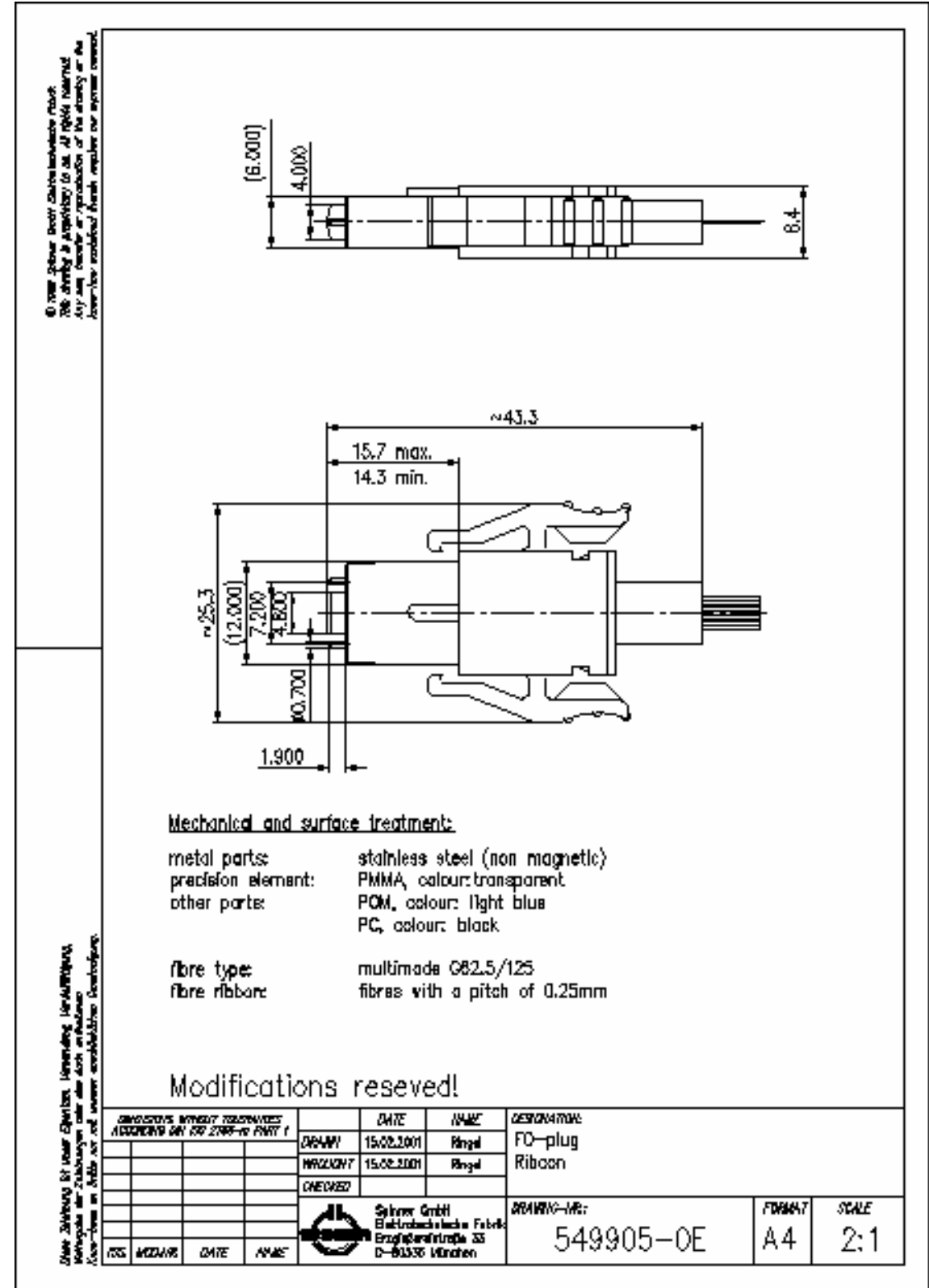

Abbildung A.1: Stecker zur Kopplung der sensorseitigen Detektionsfasern mit den spektrometerseitigen Fasern (CeramOptec) 


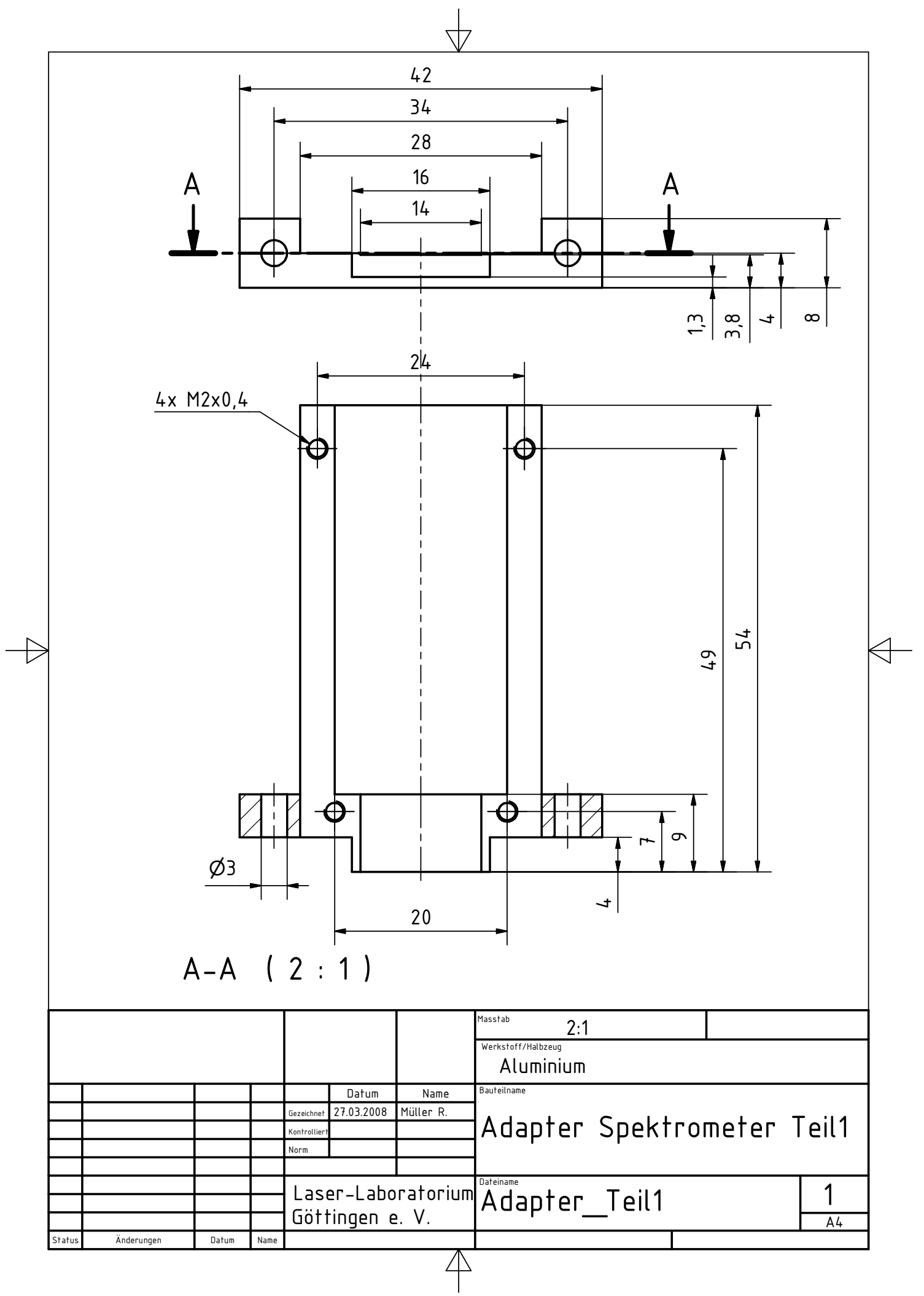

Abbildung A.2: Bauteil 1: Spektrometerferrule 


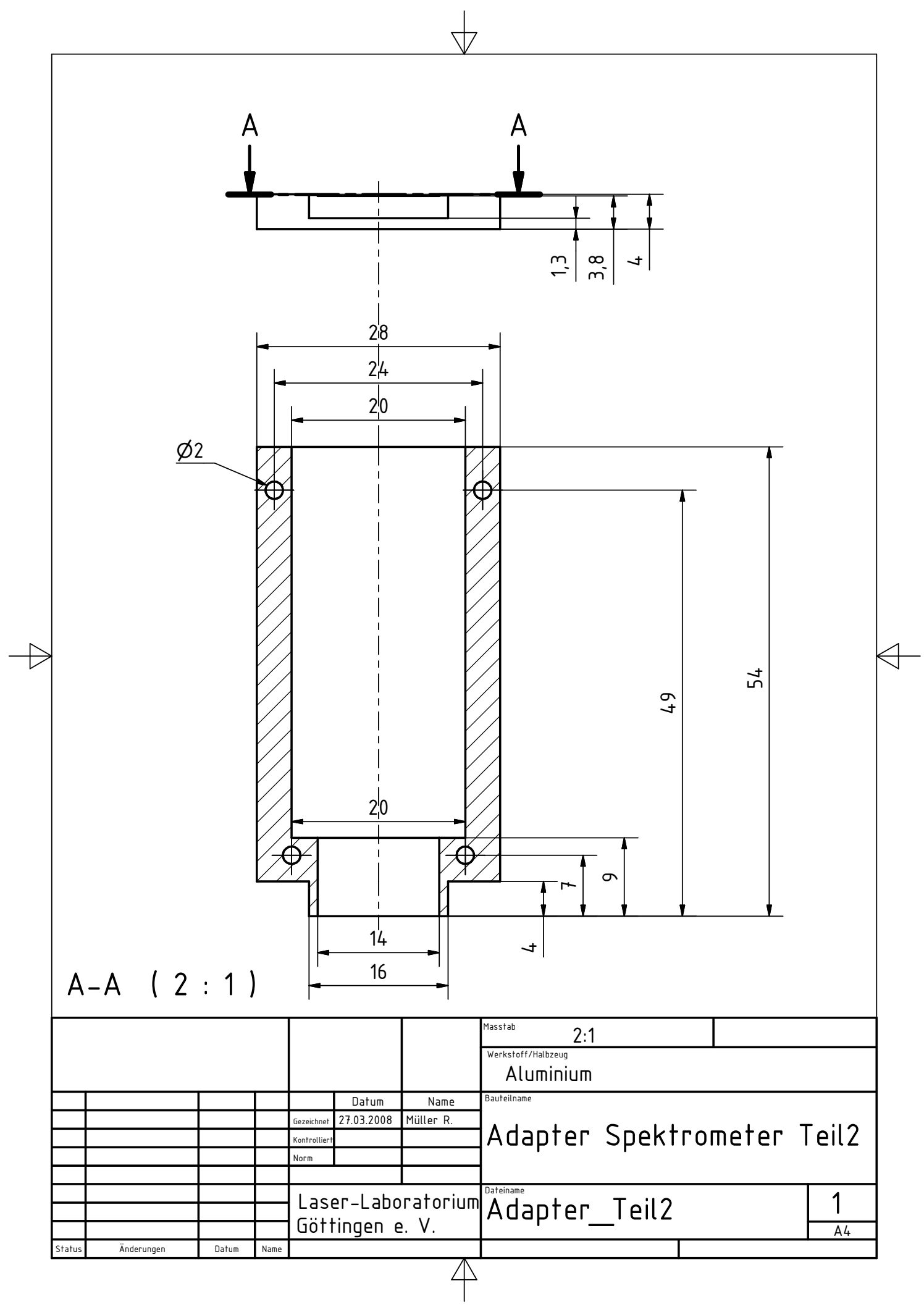

Abbildung A.3: Bauteil 2: Spektrometerferrule 


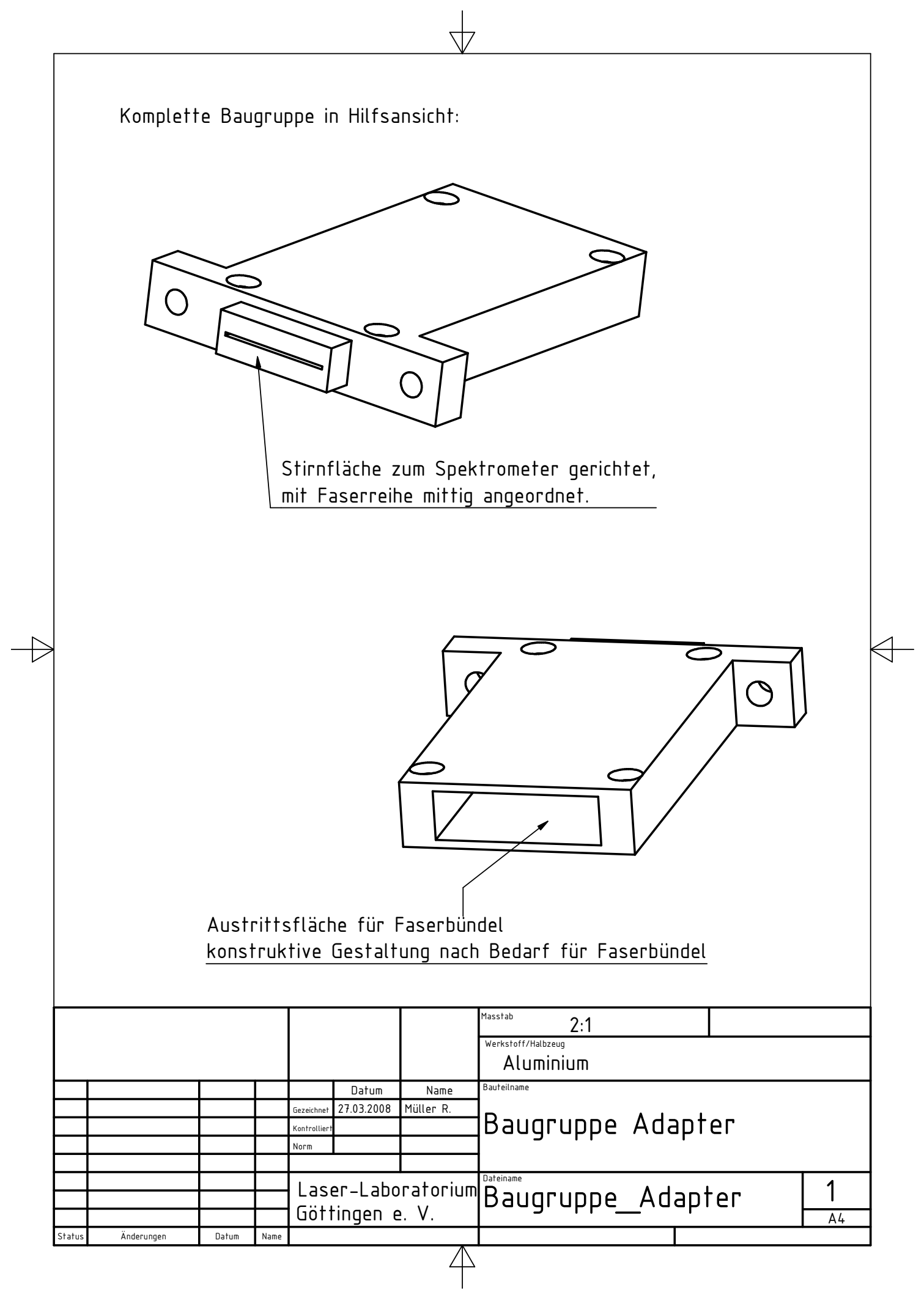

Abbildung A.4: Baugruppe Spektrometerferrule 


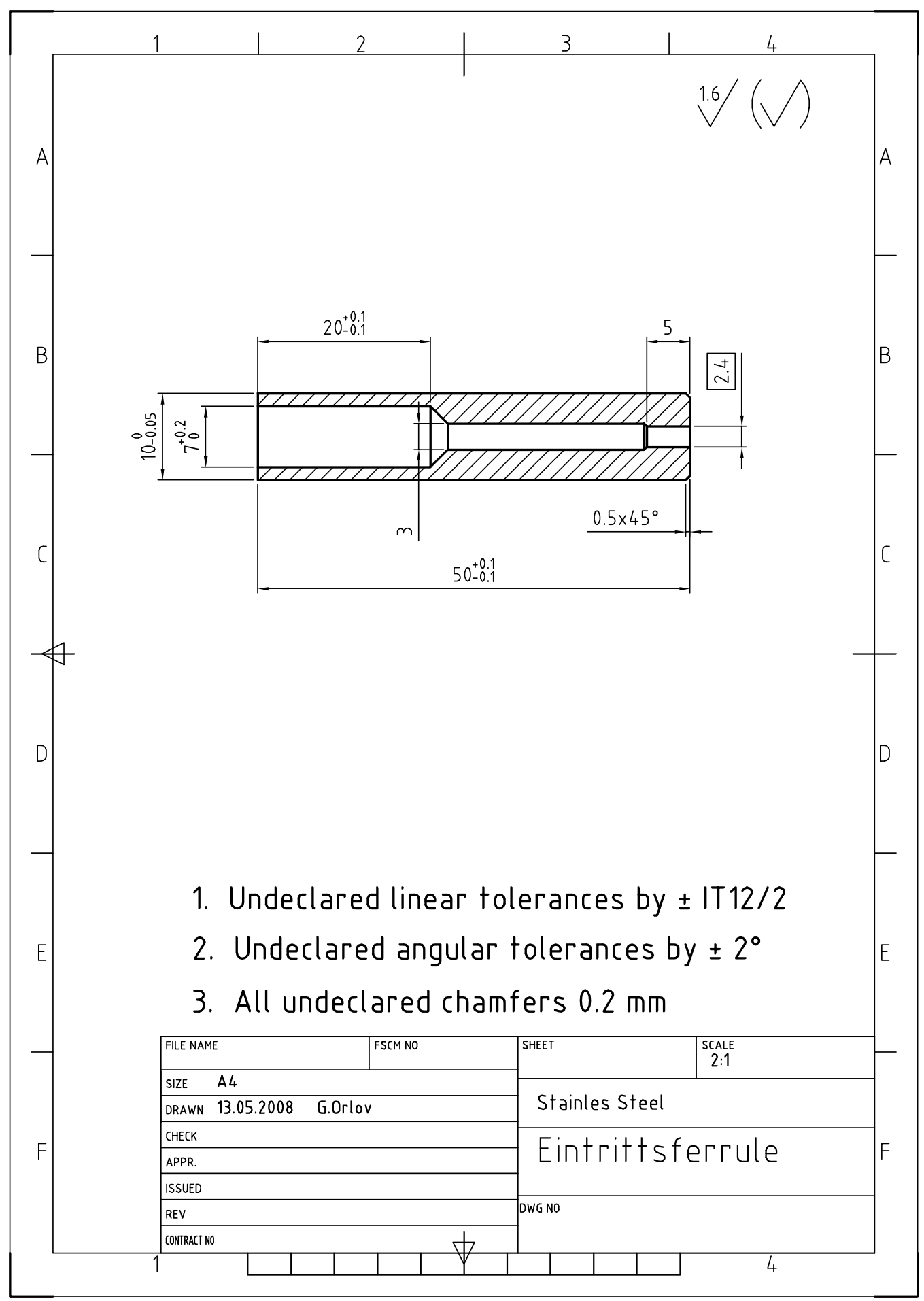

Abbildung A.5: Eintrittsferrule für Anregungsfaserbündel (konstruiert von CeramOptec) 


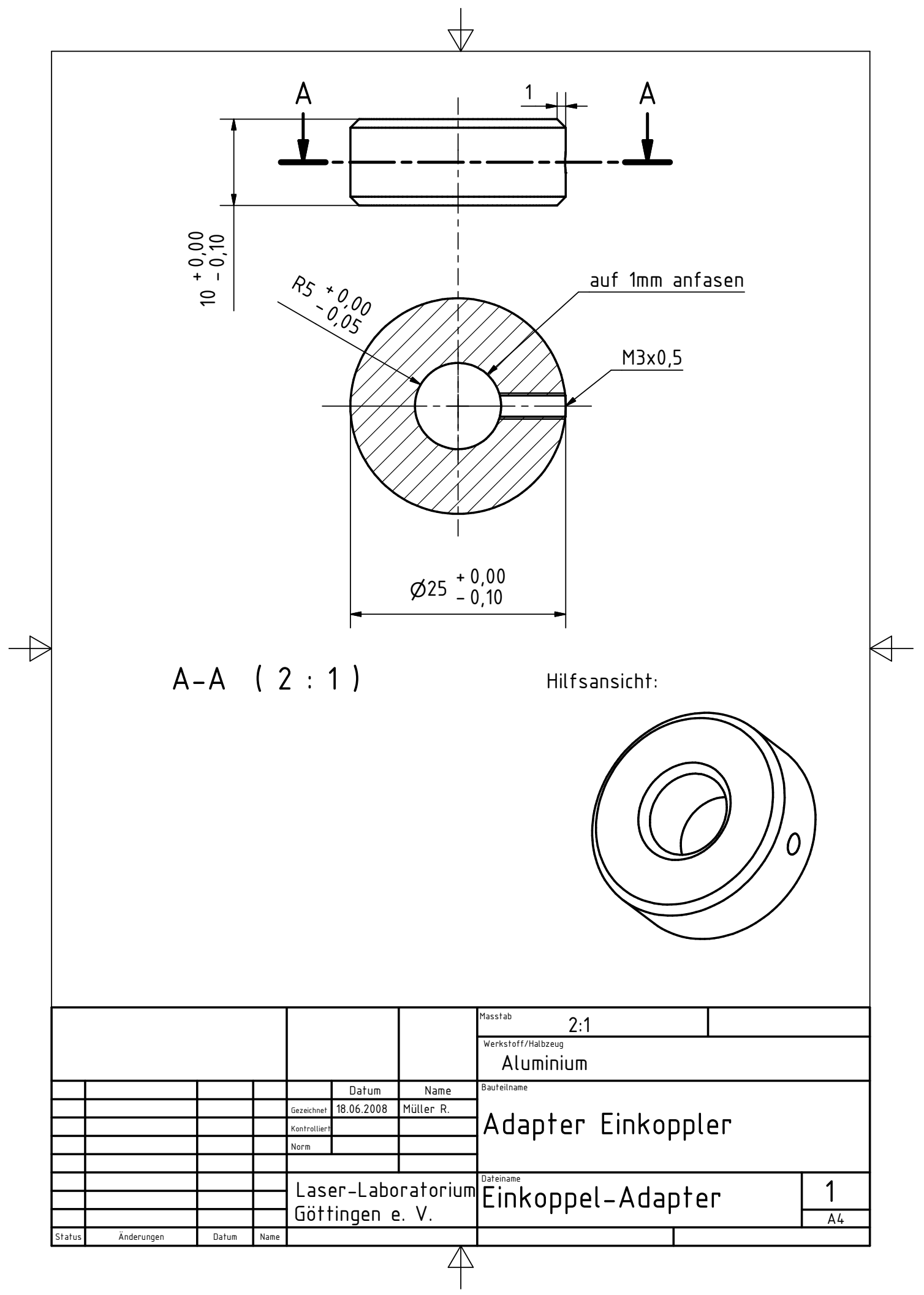

Abbildung A.6: Adapter für Eintrittsferrule für LINOS-Mikrobanksystem 


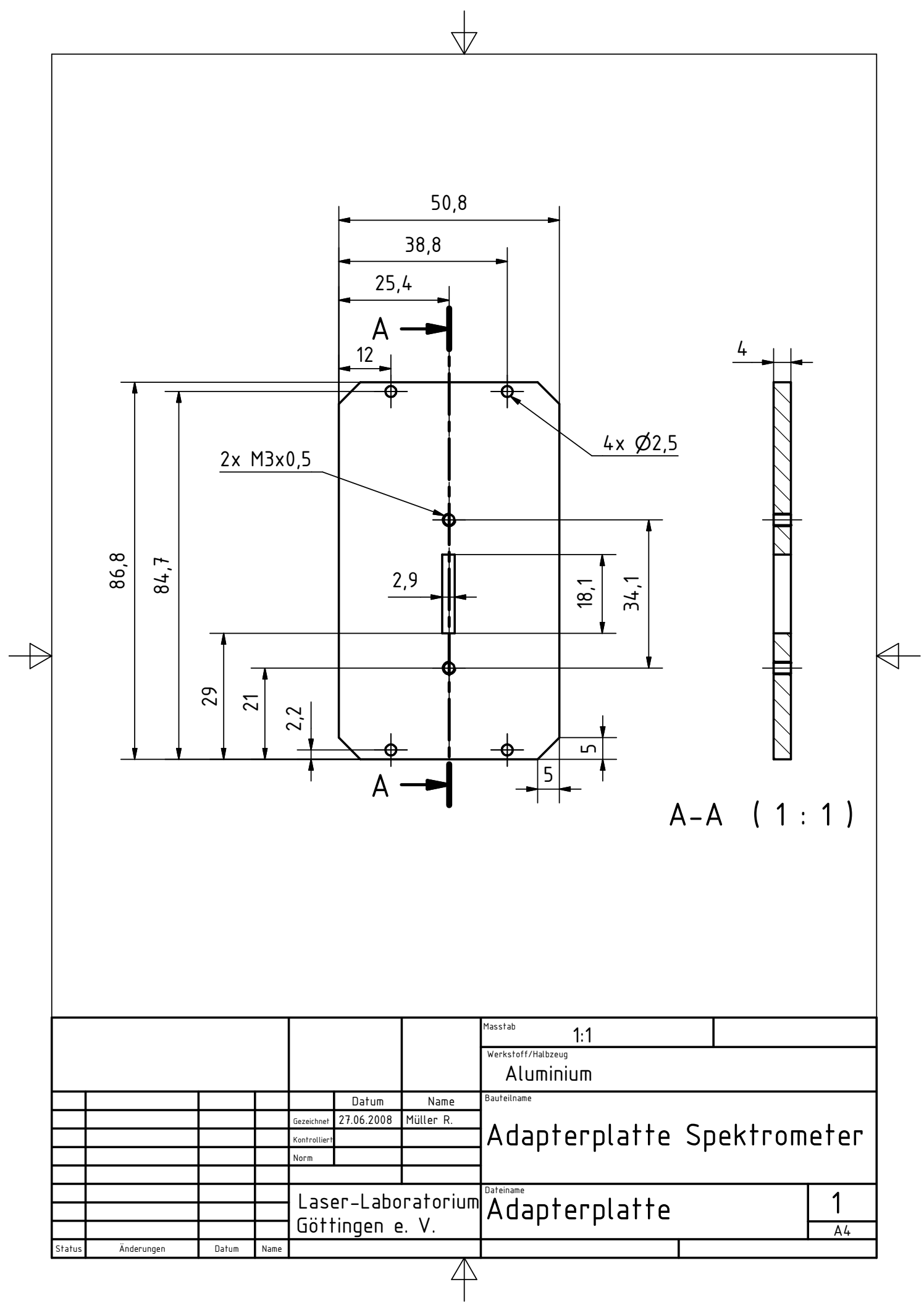

Abbildung A.7: Adapterplatte für Spektrometer 


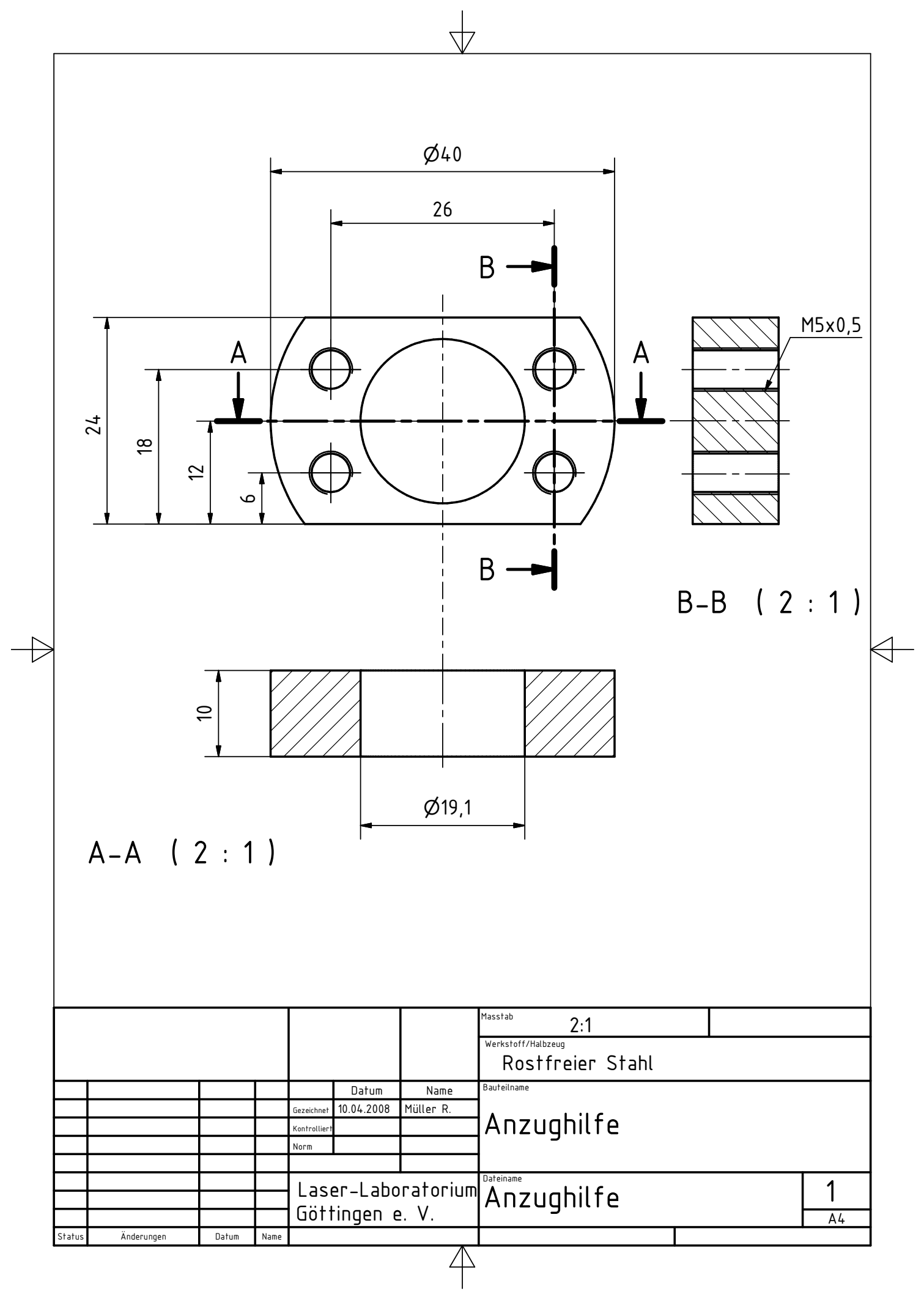

Abbildung A.8: Bauteil 1: Anzughilfe für Sensorhülle 


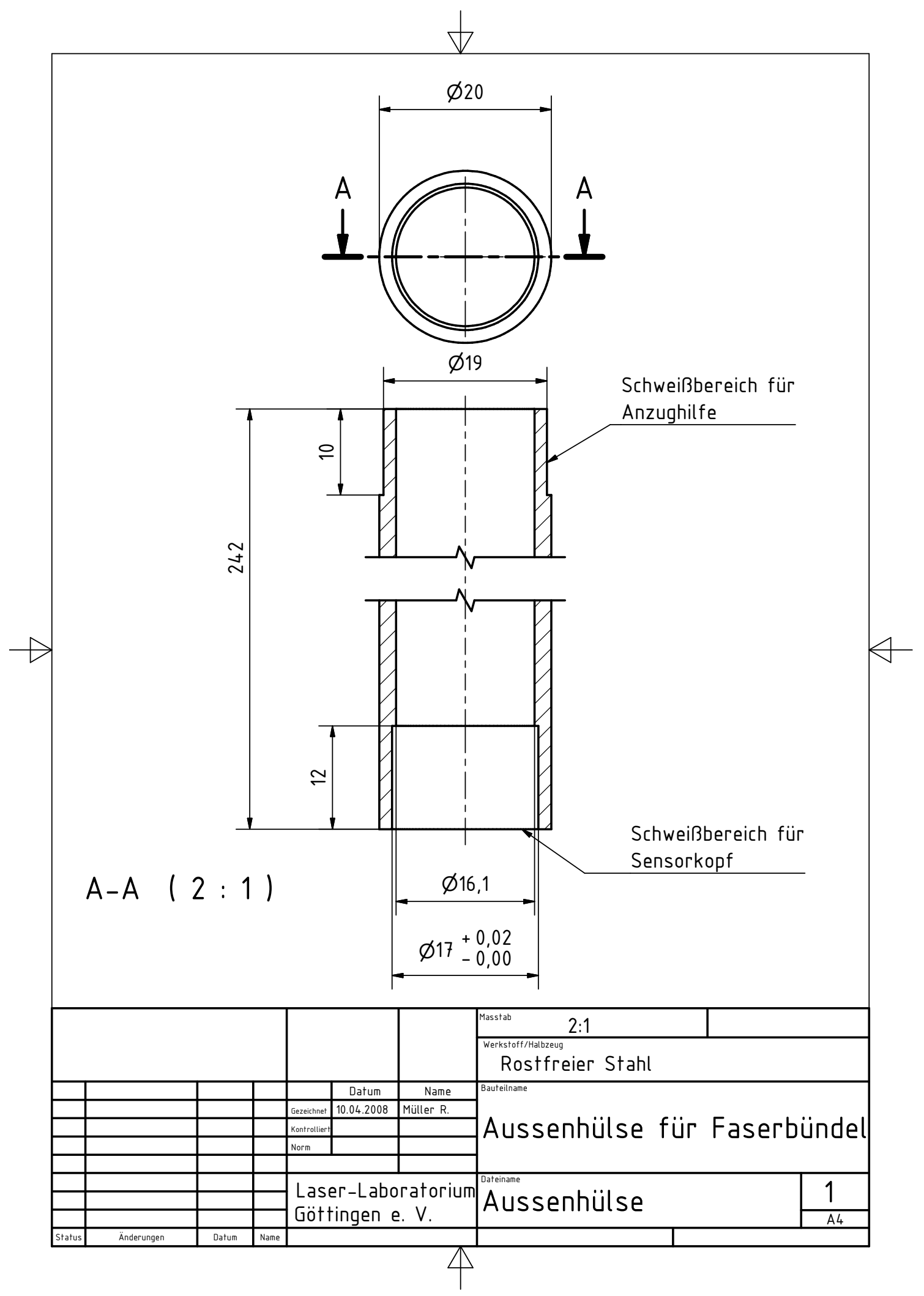

Abbildung A.9: Bauteil 2: Aussenhülse von Sensor 


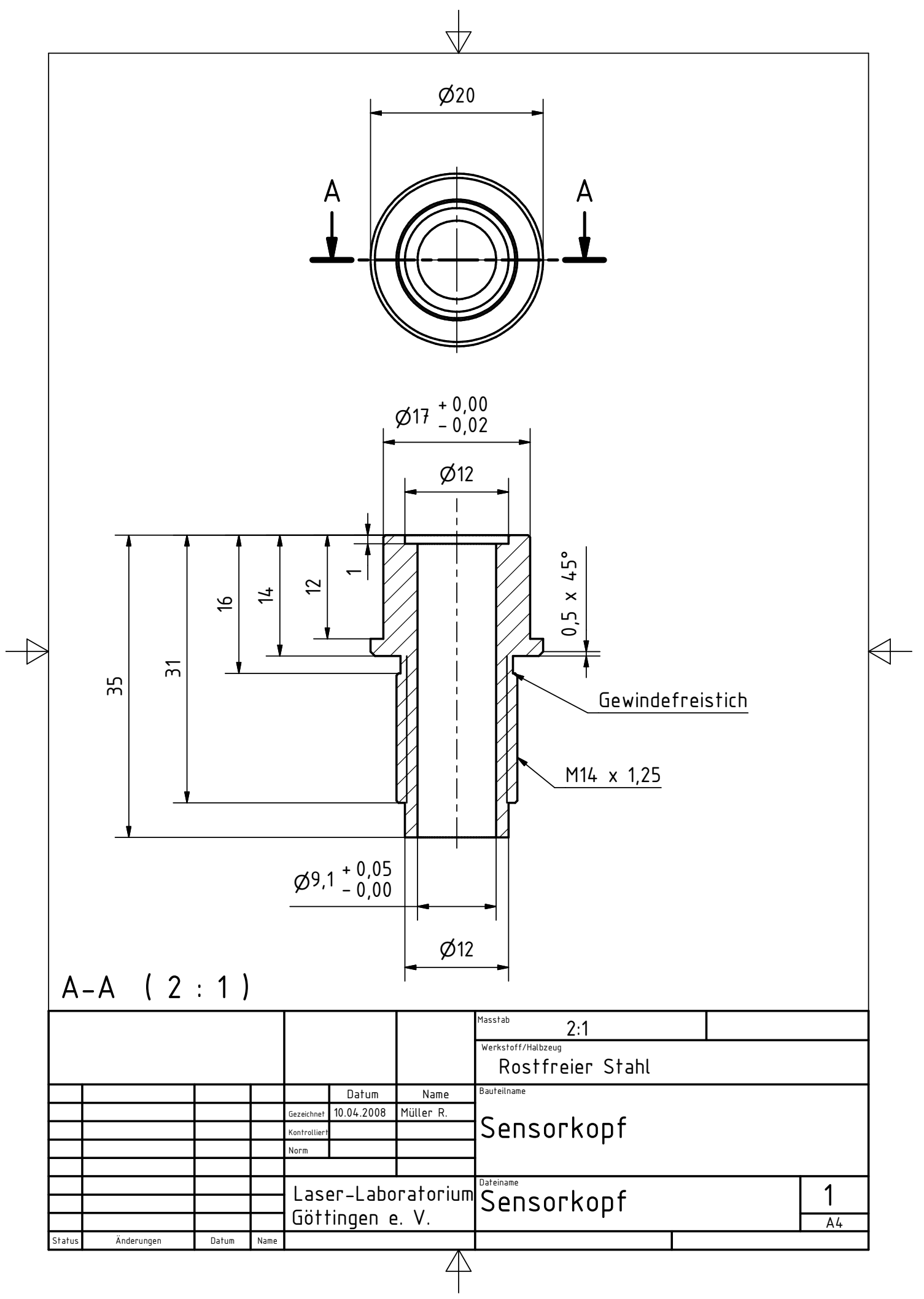

Abbildung A.10: Bauteil 3: Sensorkopf von Aussenhülse 


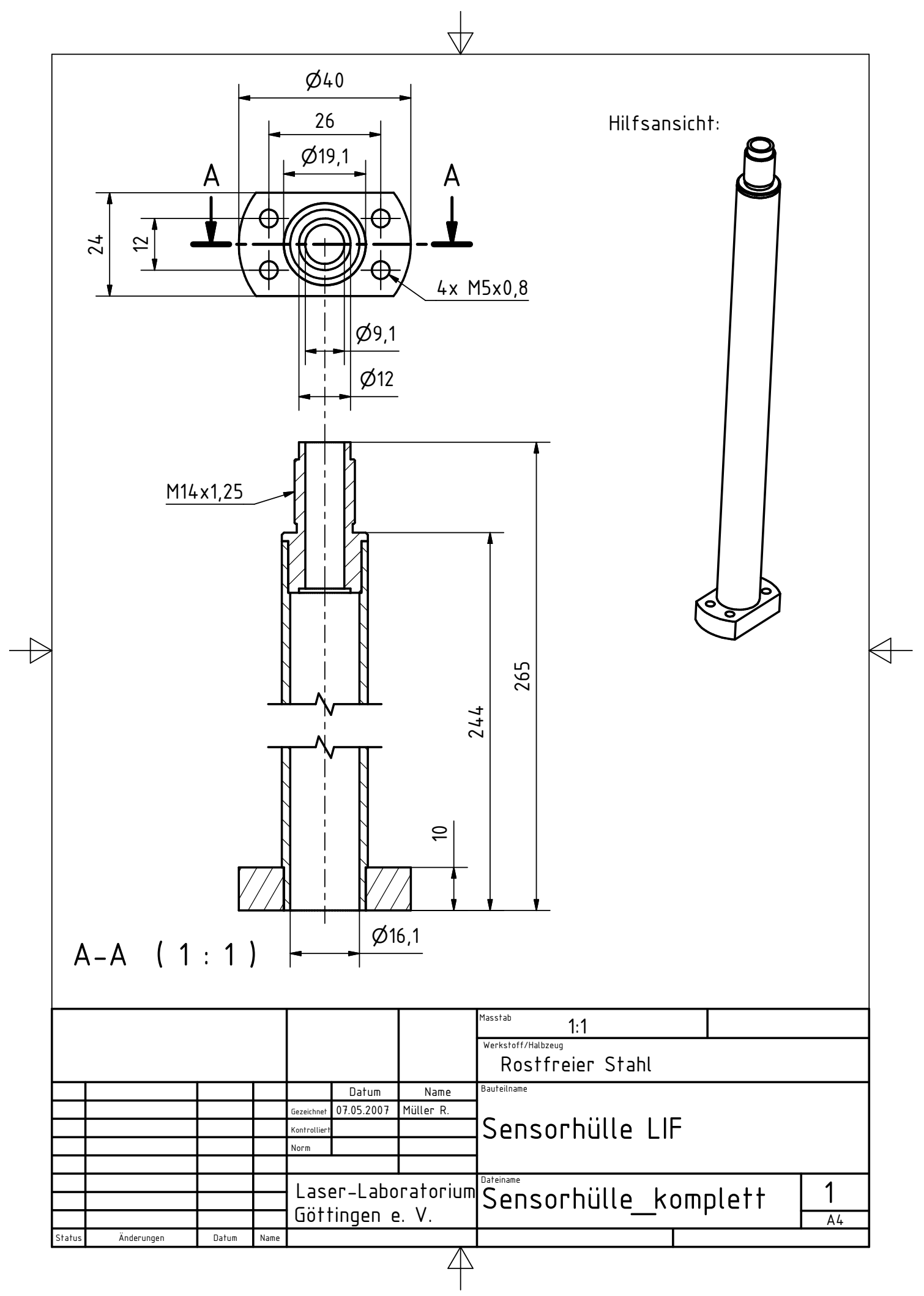

Abbildung A.11: Komplette Sensorhülse 


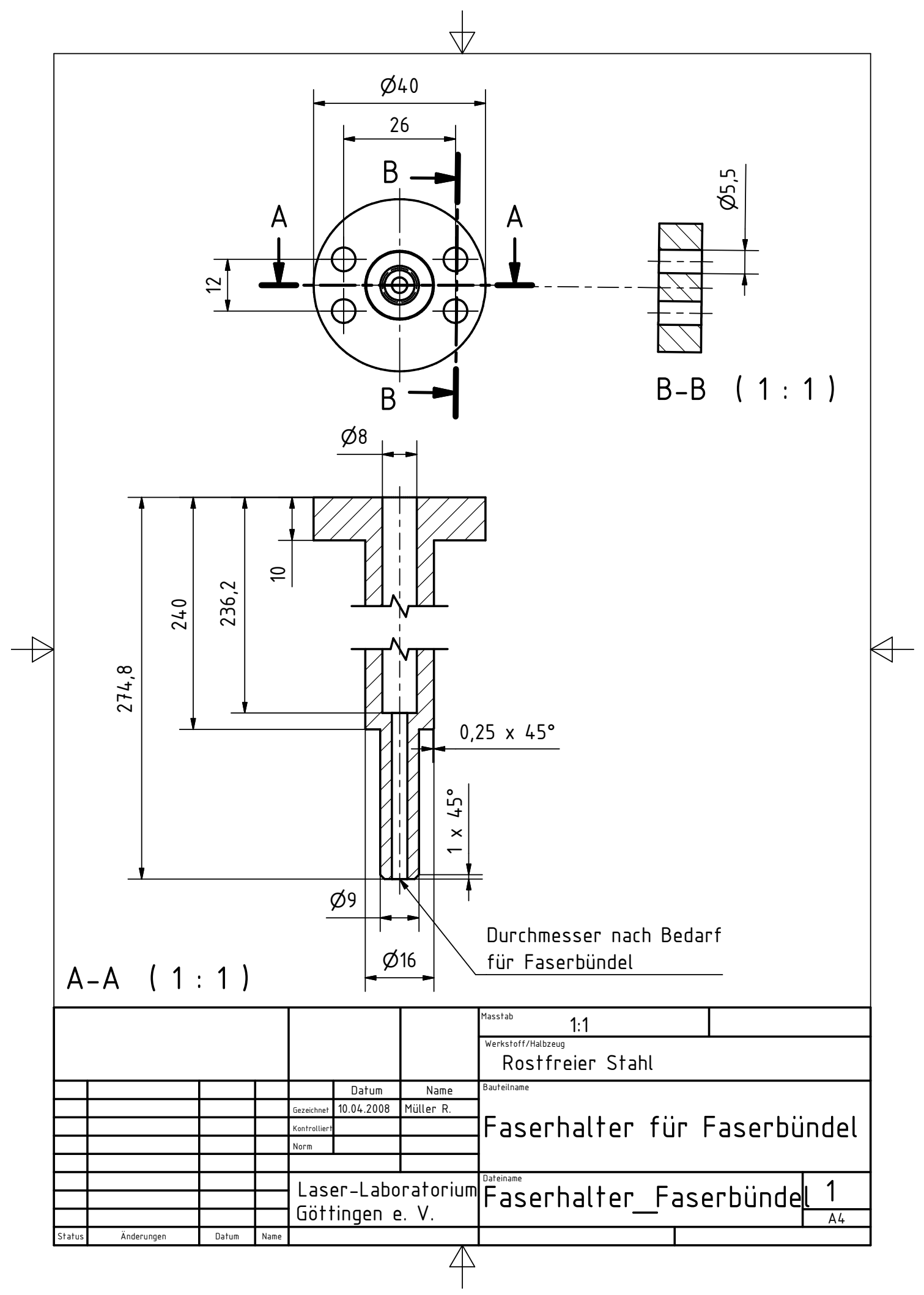

Abbildung A.12: Sensorsystem mit Aussenhülse 


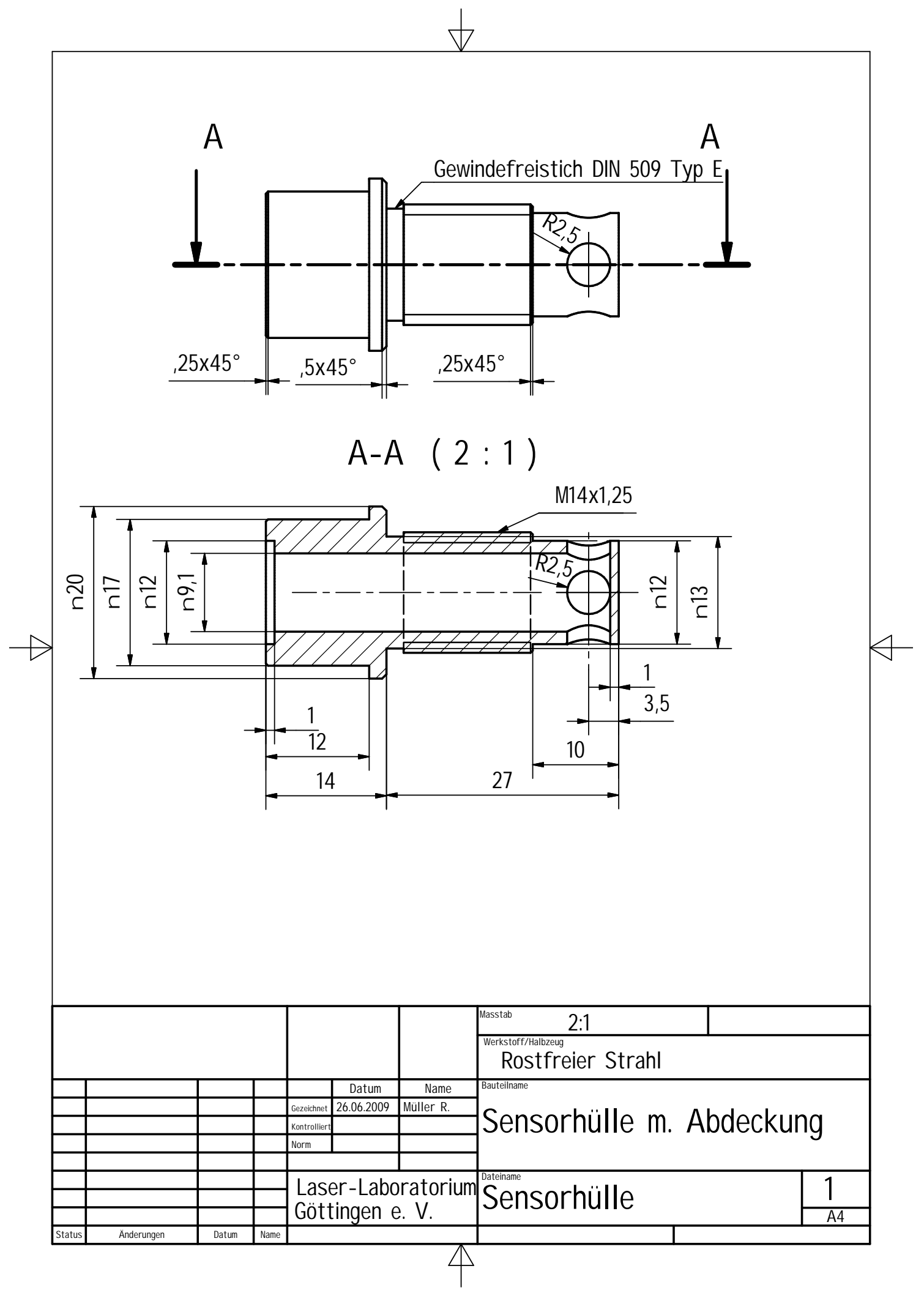

Abbildung A.13: Korrektur Sensorkopf für Motormessungen 


\section{Anhang B}

\section{Quellcode der Auswertung}

Im Folgenden ist der Quellcode des Auswertealgorithmus dargestellt. Dieser selbstgeschriebene Algorithmus ist als Macro in die Bildverarbeitungssoftware DaVis von der Firma LaVision integriert worden. 
void GlaettenBackground (string setpath, string sample, string pressure, float $\times 1$, float $\times 2$, float $\times 3$, float $\times 4$, float $\times 5$, float $\times 6$, float 1 ine1, float 1 ine2)

\{/

$/ / * * * *$ Definition der Variablen: $* * * * * * * * *$

int $d, i, j, k, 1, q, s$;

float $\mathrm{m} 1, \mathrm{~m} 2, \mathrm{~m} 3$;

float $n 1, n 2, n 3$

float $\mathrm{y}_{1}, \mathrm{y} 2, \mathrm{y} 3, \mathrm{y} 4, \mathrm{y} 5, \mathrm{y} 6$;

float area1, area2, area3;

float v395, v404, v413, vGes;

float profratio1, profratio2, profratio3, profratio4;

float $11,12, \mathrm{~s} 1, \mathrm{~s} 2, \mathrm{s3}$;

float glaett1, glaett2

string filenames, topname

string proffilenames, proftopname;

string star:

Freebuffer (0)

FreeBuffer (1)

$/ / * * * * *$ verzeichnisauswah1 \& Erzeugen des Profils: $* * * * * * * * * *$

star="a";

SetChar (star, 0, 42);

filenames=GetDirectory $($ setpath + "/" + star + ".imx", 0);

$i=1$;

topname=GetTokenN $(f j$ lenames, " $\backslash n ", i)$;

InfoText (setpath + "/Korrigierte_Profile")

MkDir(setpath + "/Korrigierte_Profile")

MkDir(setpath + "/Korrigierte_Profile/Glaettung_Profile");

while(topname $!=" ")$

LoadBuffer (setpath + "/" + topname, i)

$11=1$ inei-5

//Aufweiten des Profil-Intervalls für gleitenden

$12=1$ ine $2+5$ Mittelwert

TakeProfi 1

$(1, i, 1,11,12)$

$/ / * * * *$ Glätten des Profils (Spektrums) : $* * * * * * * * *$

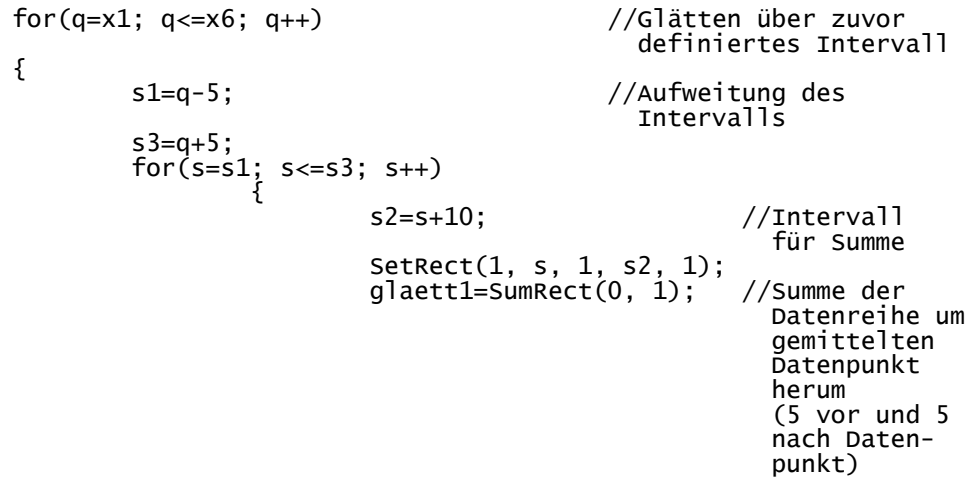




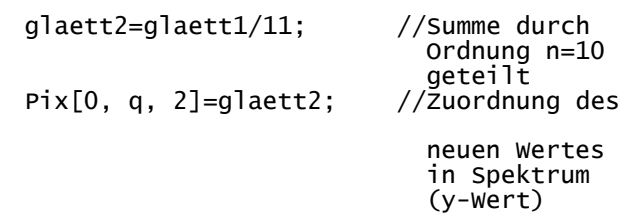

\}

StoreProfile(2, setpath + "/Korrigierte_Profile/

Glaettung_Profile/Profi1-glatt" + FormatNumber("03.0f", i)

+ ".prf");

FreeBuffer(1);

//LoadProfile (1, setpath + "/Korrigierte_Profile/

Glaettung_Profile/Profi1-glatt" + FormatNumber("03.0f", i)
+ ".prf");

$/ / * * * * *$ Berechnen der Background-Geraden:**********

$\mathrm{y} 1=\operatorname{Pix}[0, \mathrm{x} 1,2]$;

$\mathrm{y} 2=\operatorname{Pix}[0, x 2,2]$

$\mathrm{y} 3=\operatorname{Pix}[0$,
$\mathrm{y} 4=\mathrm{Pix}[0$,

$\mathrm{y} 5=\operatorname{Pix}[0, \mathrm{x} 5,2]$

y $6=\operatorname{Pix}[0, x 6,2]$

$\mathrm{m} 1=(\mathrm{y} 2-\mathrm{y} 1) /(\mathrm{x} 2-\mathrm{x} 1)$;

$\mathrm{m} 2=(y 4-y 3) /(x 4-x 3) ;$
$\mathrm{m} 3=(y 6-y 5) /(x 6-x 5) ;$

$\mathrm{n} 1=\mathrm{y} 1-\mathrm{m} 1 * \mathrm{x} 1$;

$\mathrm{n} 2=\mathrm{y} 3-\mathrm{m} 2 * x 3$

$\mathrm{n} 3=\mathrm{y} 5-\mathrm{m} 3 * \times 5$

$/ / * * * *$ Abzug des Backgrounds \& Speichern des korrigierten Profi1s:*****

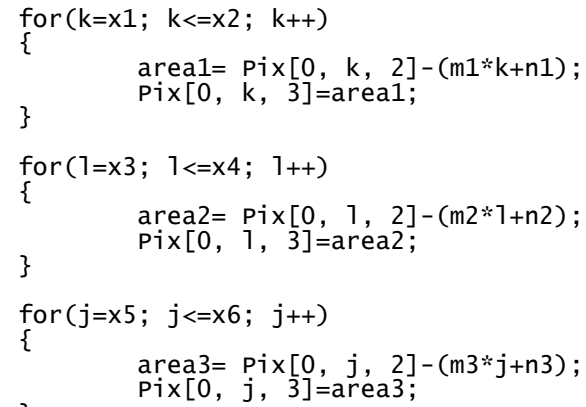




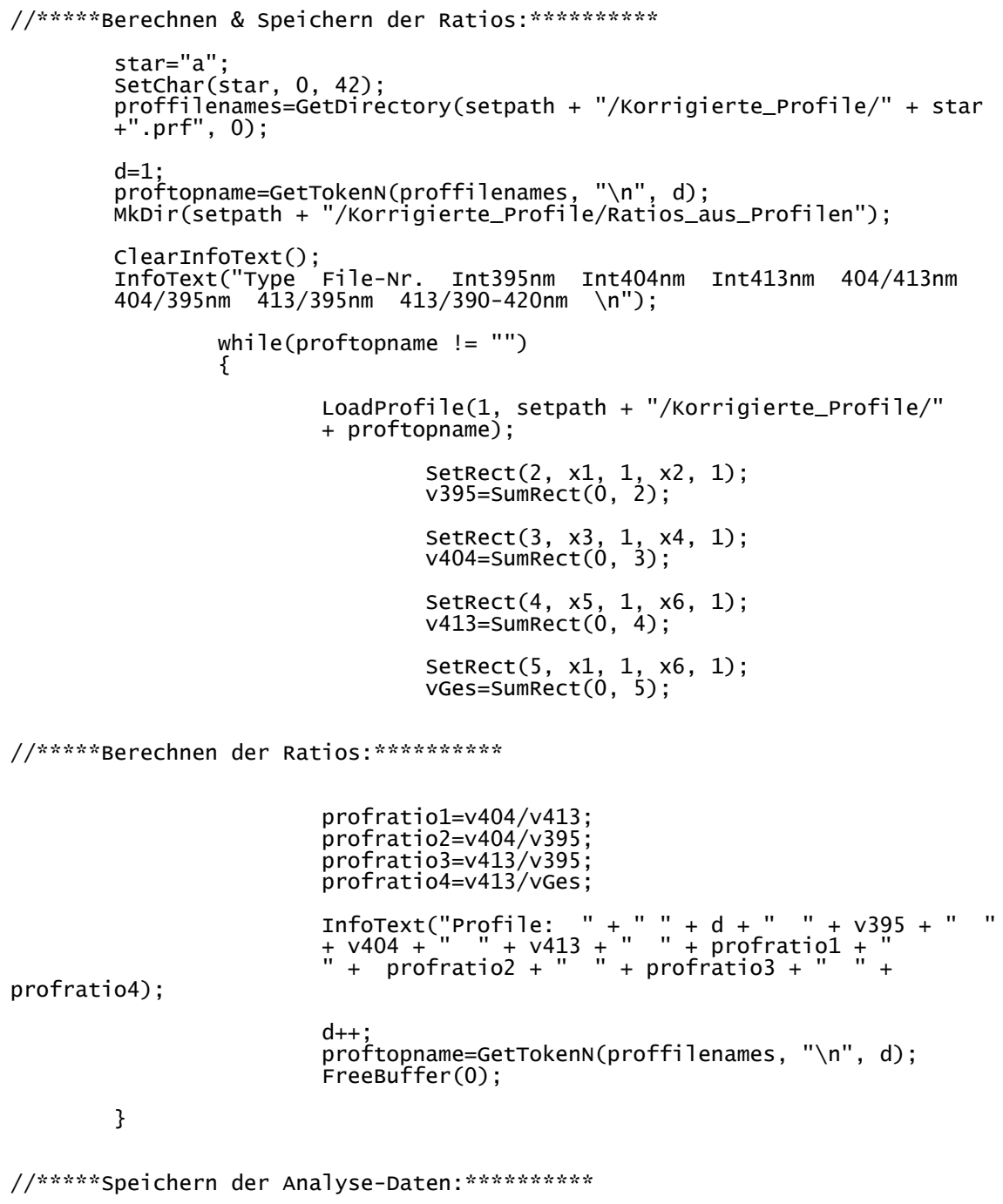

SaveInfoText(setpath + "/Korrigierte_Profile/Ratios_aus_Profilen /KorrProfRat_" + sample + "_" + pressure

+ "bar.txt");

clearinfoText ();

InfoText("Interva11e: " + "x1=" + x1 + "; $x 2="$ + x2 + "; $x 3="$

$+\times 3+" ; x 4="+x 4+" ; x 5="+x 5$

InfoText ("Integrationsbereich: $y 1="$ + 1ine1 + "; y2=" + 1ine2 + "\n");

SaveInfoText(setpath + "/Korrigierte_Profile/Ratios_aus_Profilen

/Analyse-Interval1e_" + sample + "-"

\}

+ pressure + "bar.txt"); 


\section{Abbildungsverzeichnis}

2.1 Entwicklung der Euro-Norm . . . . . . . . . . . . . . . . 4

3.1 Prinzip des Viertakt-Ottomotors . . . . . . . . . . . . . . . . . . 11

3.2 Prinzip des HCCI-Motors . . . . . . . . . . . . . . . 16

4.1 Franck-Condon-Prinzip . . . . . . . . . . . . . . . . . . . . . 19

4.2 Jablonski-Diagramm . . . . . . . . . . . . . . . . . . . . . . . . . 21

4.3 Prinzip der Schwingungsumverteilung . . . . . . . . . . . . . . . . . 24

4.4 Strukturformel von Formaldehyd . . . . . . . . . . . . . . . . . 27

4.5 Schwingungsmoden von Formaldehyd . . . . . . . . . . . . . . 28

4.6 Absorptionsspektrum von Formaldehyd . . . . . . . . . . . . . . . . . 29

4.7 Emissionsspektrum von Formaldehyd . . . . . . . . . . . . . . . . 30

5.1 Struktur der Faser . . . . . . . . . . . . . . . . . . . 34

5.2 Begriff der Totalreflexion . . . . . . . . . . . . . . . . . . . 35

5.3 Begriff der Numerischen Apertur . . . . . . . . . . . . . . . . 36

5.4 Begriff der Modendispersion . . . . . . . . . . . . . . . 37

5.5 Fasertypen . . . . . . . . . . . . . . . . . . . 37

5.6 Nicht-abbildender Homogenisator . . . . . . . . . . . . . . . 39

5.7 Prinzip bildverstärkte CCD-Kamera . . . . . . . . . . . . . . . . 42

5.8 Zeitfenster einer bildverstärkten CCD-Kamera . . . . . . . . . . . . . 43

5.9 Prinzip Czerny-Turner Monochromator . . . . . . . . . . . . . . . 44

5.10 Prinzip F-Zahl . . . . . . . . . . . . . . . . . . . . . . 45

5.11 Schematischer Aufbau des Detektorsystems . . . . . . . . . . . . 46

5.12 Transmissionskurven spektraler Filter . . . . . . . . . . . . . . . . 47

5.13 Meßprinzip am Transparentmotor . . . . . . . . . . . . . . . . 50

5.14 Prinzip des Sensors der 1. Generation . . . . . . . . . . . . . 51

5.15 Struktur des Sensors der 2. Generation . . . . . . . . . . . . 53

5.16 Anwendung des Sensors im Motor . . . . . . . . . . . . . . . 54

6.1 Schema Druckkammer . . . . . . . . . . . . . . 56

6.2 Triggerschema PTU9 . . . . . . . . . . . . . . . . . 57

6.3 Versuchsaufbau Druckkammer . . . . . . . . . . . . . . . 58

6.4 Temperaturabhängiges Emissionsspektrum von Formaldehyd . . . . . 59

6.5 Temperaturabhängige Fluoreszenzlebensdauer von Formaldehyd . . . 61 
6.6 Thermalisierungskurven von Formaldehyd . . . . . . . . . . . . . . 62

6.7 Fluoreszenzstabilität von Formaldehyd . . . . . . . . . . . . . . 63

6.8 Transmission des Faserbündels . . . . . . . . . . . . . . . . . . . . 64

6.9 Spektrum mit Glättungsfunktion . . . . . . . . . . . . . . 69

6.10 Integrationsempfindlichkeit des Verfahrens . . . . . . . . . . . . . . 71

6.11 Arrheniusplots von Formaldehyd/Luft . . . . . . . . . . . . . . 73

6.12 Arrheniusplot: Einfluss von Isooktan . . . . . . . . . . . . . . . . . 74

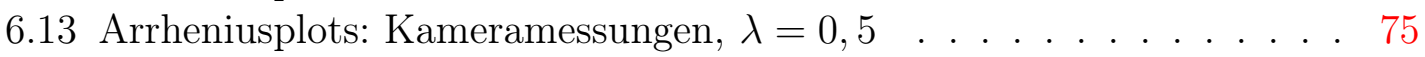

6.14 Arrheniusplots: Kameramessungen, $\lambda=1 \ldots \ldots$. . . . . . . . 75

6.15 Arrheniusplots: Kameramessungen, $\lambda=1,5 \ldots \ldots$. . . . . . . 76

6.16 Sensoreinbau in Druckkammer . . . . . . . . . . . . . . . 77

6.17 Schematischer Aufbau mit Sensor 1 . . . . . . . . . . . . . . . 78

6.18 Arrheniusplots gemessen mit Sensor 1 . . . . . . . . . . . . . . 78

6.19 Schematischer Aufbau mit Sensor 2 . . . . . . . . . . . . . . . . 79

6.20 Arrheniusplots gemessen mit Sensor 1 . . . . . . . . . . . . . . 80

6.21 Kurbelwinkelaufgelöste Druckkurve . . . . . . . . . . . . . . . 83

6.22 Kurbelwinkelaufgelöste Druck- und Temperaturkurve . . . . . . . . . 88

A.1 Steckverbindung für Fasern . . . . . . . . . . . . . . . 96

A.2 Bauteil 1: Spektrometerferrule . . . . . . . . . . . . . . 97

A.3 Bauteil 2: Spektrometerferrule . . . . . . . . . . . . . . . . . 98

A.4 Baugruppe Spektrometerferrule . . . . . . . . . . . . . . . . . . . 99

A.5 Eintrittsferrule für Anregungsfaserbündel (CeramOptec) . . . . . . . 100

A.6 Adapter für Eintrittsferrule für LINOS-Mikrobanksystem . . . . . . . 101

A.7 Adapterplatte für Spektrometer . . . . . . . . . . . . . . . . . . 102

A.8 Bauteil 1: Anzughilfe für Sensorhülle . . . . . . . . . . . . . . . . 103

A.9 Bauteil 2: Aussenhülse von Sensor . . . . . . . . . . . . . . . . . . . . 104

A.10 Bauteil 3: Sensorkopf von Aussenhülse . . . . . . . . . . . . . . . . 105

A.11 Komplette Sensorhülse . . . . . . . . . . . . . . . . . 106

A.12 Sensorsystem mit Aussenhülse . . . . . . . . . . . . . . . . . 107

A.13 Korrektur Sensorkopf für Motormessungen . . . . . . . . . . . . . . 108 


\section{Literaturverzeichnis}

[1] Nicolaus August Otto. Gasmotor. Patentschrift Nr. 532, 1877. 4

[2] Arnold Langen. Nicolaus August Otto. Schöpfer des Verbrennungsmotors. Franckh, 1949. 4

[3] Rudolf Diesel. Arbeitsverfahren und Ausführungsart für Verbrennungskraftmaschinen. Patentschrift Nr. 67207, 1893. 4

[4] Rudolf Diesel. Die Entstehung des Dieselmotors. Springer Verlag, 1913. 4

[5] J. Warnatz, U. Maas, and R.W. Dibble. Verbrennung, Physikalisch-Chemische Grundlagen, Modellierung und Simulation, Experimente, Schadstoffentstehung. Springer Verlag, 2001. 8

[6] E. Bach and P. Pfeiffer. Lehrbrief V6, Teil1: Ottomotoren-Gemischbildung, Zündung und Verbrennung im Ottomotor. Technical report, Hochschule für Technik und Wirtschaft Dresden (FH), 2005. 8, 13

[7] R. Pischinger, G. Kraßing, G. Taučar, and Th. Sams. Thermodynamik der Verbrennungskraftmaschine. Band 5, Springer Verlag, 1989. 9, 12

[8] F. Pischinger. Abschlussbericht SFB 224: Motorische Verbrennung. Technical report, 2001. 9

[9] E. Bach and P. P. Pfeiffer. Lehrbrief V6, Teil2: Ottomotoren-Gemischbildung, Zündung, und Verbrennung. Technical report, Hochschule für Technik und Wirtschaft, 2005. 9, 12

[10] Hermann Teegen. Verfahren zum Betrieb von gemischverdichtenden, selbstzündenden Zweitakt-Brennkraftmaschinen. Patentschrift Nr. 823233, 1951. 14

[11] Hermann Teegen. Gemischverdichtete Zweitakt-Verbrennungskraftmaschine mit einstellbarer Kompression und Selbstzündung. Patentschrift Nr. 864476, 1952. 14

[12] S. Onishi, S. Hong Jo, P. Do, and S. Kato. Active thermo-atmosphere combustion (ATAC) - a new combustion process for internal combustion engines. Society of Automotive Engineers (SAE) technical paper, 790501, 1979. 14 
[13] Ulrich Spicher. Ottomotor mit Direkteinspritzung: Verfahren, Systeme, Entwicklung, Potenzial. Vieweg Verlag, 2007. 15

[14] C. Schulz, V. Sick, J. Wolfrum, V. Drewes, M. Zahn, and R. Maly. Quantitative 2D single-shot imaging of $\mathrm{OH}$ concentrations and temperatures in a transparent SI engine. 26th Symp. (Int.) on Combustion, the Combustion Institute, Pittsburgh, page 2597, 1996. 17

[15] A. Zur Loye and D.A. Santavicca. Temperature and concentration measurements in an internal combustion engine using laser Raman spectroscopy. AIAA 18th Thermophysics Conference, 83:1551, 1983. 17

[16] J.L. Palmer, B.K. McMillan, and R. K. Hanson. Multi-line fluorescence imaging of the rotational temperature field in an shock-tunnel free jet. Appl. Phys., 63:167, 1996. 17

[17] A. Arnold, B. Lange, T. Bouché, T. Heitzmann, G. Schiff, P. Ketterle, P. Monkhouse, and J. Wolfrum. Absolute temperature fields in flames by 2D-LIF of OH using excimer lasers and CARS spectroscopy. Ber. Bunsenges. Phys. Chem., 96:1388, 1992. 18

[18] S. Einecke, C. Schulz, and V. Sick. Measurement of temperature, fuel concentration and equivalence ratio fields using tracer LIF in IC engine combustion. Appl. Phys., B 71:717-723, 2000. 18

[19] R. Müller and V. Beushausen. Two-Line LIF Emission Thermometry for GasTemperature Determnation in IC -Engines. Advanced Microsystems for Automotive Applications, Springer, Berlin:127, 2008. 18

[20] Jeanne L. McHale. Molecular Spectroscopy. Prentice-Hall, Inc., 1999. 23

[21] Irvin Glassman. Combustion. Academic Press Inc., 1987. 25

[22] B. Bäuerle, F. Hoffmann, F. Behrendt, and J. Warnatz. Detection of Hot Spots in the End Gas of an IC Engine Using Two-Dimensional LIF of Formaldehyde. Proc. Comb. Inst., 25:135, 1995. 26

[23] Senatskommission zur Prüfung gesundheitsschädlicher Arbeitsstoffe. MAKund BAT-Werte-Liste 2008. DFG Deutsche Forschungsgemeinschaft, 2008. 27

[24] D.J. Clouthier and D.A. Ramsay. The spectroscopy of formaldehyde and thioformaldehyde. Ann. Rev. Phys. Chem., 34:31-58, 1983. 27, 28

[25] Robert J. H. Klein-Douwel, Jorge Luque, Jay B. Jeffriesand Gregory P. Smith, and David R. Crosley. Laser-induced fluorescence of formaldehyde hot bands in flames. Applied Optics, 39:3712-3715, 2000. 27 
[26] Joel E. Harrington and Kermit C. Smyth. Laser-induced fluorescence measurements of formaldehyde in a methane/air diffusion flame. Chemical Physics Letters, 202:196-202, 1993. 27

[27] R. Bombach and B. Käppeli. Simultaneous visualisation of tranient species in flames by planar-laser-induced fluorescence using a single laser system. Applied Physics, B 68:251-255, 1999. 27

[28] K. H. Becker, U. Schurath, and T. Tatarczyk. Fluorescence Determination of Low Formaldehyde Concentrations in Air by Dye Laser Excitation. Applied Optics, 14, No. 2:310-313, 1975. 27

[29] G.R. Möhlmann. Formaldehyde detection in air by laser-induced fluorescence. Applied Spectroscopy, 39:98-101, 1985. 27

[30] G. H. Dieke and G. B. Kistiakowsky. The Structure of the Ultraviolet Absorption Spectrum of Formaldehyde. Physical Review, 45:4-28, 1934. 27

[31] C. Bradley Moore and James C. Weisshaar. Formaldehyde photochemistry. Ann. Rev. Phys. Chem., 34:525-555, 1983. 27, 28

[32] D. C. Moule and A. D. Walsh. Ultraviolet Spectra and Excited States of Formaldehyde. Chemical Reviews, 75, No.1:67-84, 1975. 27

[33] Gerhard Herzberg. Molecular spectra and molecular structure Band 2: Infrared and Raman spectra of polyatomic molecules. New York, 1947. 28

[34] Jerry D. Rogers. Ultraviolet Absorption Cross Section and Atmospheric Photodissociation Rate Constants of Formaldehyde. J. Phys. Chem., 94:4011-4015, 1990. 29

[35] C. Brackmann, J. Nygren, X. Bai, Z. Li, H. Bladh, B. Axelsson, I. Denbratt, L. Koopmans, P.E. Bengtsson, and M. Aldén. Laser-induced fluorescence of formaldehyde in combustion using third harmonic Nd:YAG laser excitation. Spectrochimica Acta Part A, 59:3347-3356, 2003. 30

[36] J.C. Weisshaar, A.P. Baronavski, A. Cabello, and C. Bradley Moore. Collisionless decay, vibrational relaxation, and intermediate case quenching of $S_{1}$ formaldehyde. J. Chem. Phys., 69(11):4720-4731, 1978. 30

[37] Thomas Metz, Xiao Bai, Frederik Ossler, and Marcus Aldén. Fluorescence lifetimes of formaldehyde $\left(\mathrm{H}_{2} \mathrm{CO}\right)$ in the $\tilde{A}^{1} \mathrm{~A}_{2} \rightarrow \tilde{X}^{1} \mathrm{~A}_{1}$ band system at elevated temperatures and pressures. Spectrochimica Acta Part A, 60:1043-1053, 2004. 30 
[38] A. Burkert and W. Triebel. Temperaturmessungen bis 800K in Modellapparaturen und Verbrennungsmotoren basierend auf laser-induzierter Fluoreszenz von Formaldehyd. Berichte zur Energie- und Verfahrenstechnik BEV, Tagung Motorische Verbrennung- Aktuelle Probleme und moderne Lösungsansätze, Haus der Technik, Erlangen:263-271, 2003. 31

[39] Kazuhiko Shibuya, Paul W. Fairchild, and Edward K.C. Lee. Single rotational level fluorescence quantum yields, radiative lifetimes, and nonradiative decay rates of $\mathrm{S}_{1} \mathrm{D}_{2} \mathrm{CO}$ and $\mathrm{H}_{2} \mathrm{CO}\left(\tilde{A}^{1} \mathrm{~A}_{2}, 4^{1}\right)$ : Rotational dependence. J. Chem. Phys., 75:3397-3406, 1981. 31

[40] Kazuhiko Shibuya and Edward K.C. Lee. Vibrotional and electronic energy transfer from a single vibronic level of $H_{2} C O\left(\tilde{A},{ }^{1} A_{2}\right)$, the $4^{1}$ level. J. Chem. Phys., 69(2):758-766, 1978. 31

[41] Werner Daum, Jürgen Krauser, Peter E. Zamzow, and Olaf Ziemann. POF Optische Polymerfasern für die Datenkommunikation. Springer Verlag, 2001. 37

[42] T. Schmidt-Uhlig, P. Karlitschek, G. Marowsky, and Y. Sano. New simplified coupling scheme for the delivery of $20 \mathrm{MW}$ Nd:YAG laser pulses by large core optical fibers. Appl. Phys., B 72:183-186, 2001. 39

[43] B. P. Varma and C. Ghosh. Some optical properties of a multialkali (S20) photocathode and the processing parameters. J. Phys. D: Appl. Phys., 6:628632, 1973. 41

[44] S. Hallensleben, S.W. Harmer, and P.D. Townsend. Optical constants for the S20 photocathode, and their application to increasing photomultiplier quantum efficiency. Optics Communications, 180:89-102, 2000. 41

[45] Schott Glaswerke, editor. Optische Glasfilter. Schott Glaswerke, 1988. 46

[46] G. Konig and C.G.W. Sheppard. End Gas Autoignition and Knock in a Spark Ignition Engine. SAE Paper, 902135, 1990. 48

[47] P.G. Felton, J. Mantzaras, D.S. Bomse, and R.L. Woodin. Initial TwoDimensional Laser Induced Fluorescence Measurement of $\mathrm{OH}$ Radicals in an Internal Combustion Engine. SAE Paper, 881633, 1988. 48

[48] J.B. Ghandhi, P.G. Felton, B.F. Gajdeczko, and F.V. Bracco. Investigation of the Fuel Distribution in a Two-Stroke Engine with an Air-Assisted Injector. SAE Paper, 940394, 1994. 48

[49] W.M. Scott. Understanding Diesel Combustion through the Use of High Speed Moving Pictures in Color. SAE Paper, 690002, 1969. 48 
[50] J. Dec, A.O. ZurLoye, and D.S. Siebers. Soot Distribution in a DI Diesel Engine Using 2-D Laser Induced Incandescence Imaging. SAE Paper, 910224, 1991. 48

[51] Hua Zhao and Nicos Ladommatos. Engine Combustion Instrumentation and Diagnostics. Society of Automotive Engineers Inc., Warrendale, Pa., 2001. 48, 49

[52] F.W. Bowditch. A New Tool for Combustion Research-A Quarz Piston Engine. SAE Trans., 69:17-23, 1961. 49

[53] S. Bates. A Transparent Engine for Flow and Combustion Visualisation Studies. SAE Paper, 880520, 1988. 49

[54] F. Lewitzka, M. Niederkrüger, and G. Marowsky. Application of TwoDimensional LIF for the Analysis of Aromatic Molecules in water. In P. Hering, J.P. Lay, and S. Strey, editors, Laser in Enviromental and Life Sciences, pages 141-161. Springer Verlag Berlin, 2003. 58

[55] Merck. Sicherheitsdatenblatt paraformaldehyd reinst dac. Technical report, Merck KGaA, 2003. 59

[56] Linde AG. Datenblatt Synthetische Luft. Technical report, Linde Gas, 2007. 60

[57] Reinhold Dolt. Indizierung in der Motorenentwicklung. Verlag Moderne Industrie, 2006. 82

[58] A. Grosch, V. Beushausen, O. Thiele, and R. Grzeszik. Crank Angle Resolved Determination of Fuel-Concentration and Air/Fuel Ratio in a SI-Production Engine by Using a Modified Optical Spark Plug. SAE Paper, 01-0644, 2007. 84 



\section{Danksagung}

Die vorliegende Arbeit entstand am Laser-Laboratorium Göttingen e.V. und ich möchte mich hiermit bei allen Menschen bedanken, die einen Beitrag zum Gelingen dieser Arbeit geleistet haben.

Ich danke Herrn Prof. Werner Lauterborn für die Betreuung meiner Arbeit als Hauptreferent seitens der Universität Göttingen.

Herrn Prof. Gerd Marowsky danke ich dafür, dass er es mir ermöglichte, die Dissertation am Laser-Laboratorium Göttingen e.V. durchführen zu können.

Mein besonderer Dank gilt Herrn Dr. Volker Beushausen, dem Leiter der Arbeitsgruppe Photonische Sensorik am Laser-Laboratorium Göttingen. Er stand mir immer mit kompetenten Ratschlägen zur Seite und trug in zahlreichen wissenschaftlichen Diskussionen entscheidend zum Erfolg dieser Arbeit bei.

Ebenso möchte ich mich bei Thomas Berg und Michael Kaffka von der Firma LaVision bedanken. Herr Berg war für mich stets ein Ansprechpartner bei Fragen über Kamerasysteme und durch die Erfahrungen von Herrn Kaffka habe ich viel über Konstruktion gelernt.

Ich danke auch allen meinen Kollegen am Laser-Laboratorium Göttingen für die freundschaftliche Zusammenarbeit. Sie sorgten in vielen anregenden Diskussionen für neue Denkanstöße und unterstützten mich auf unterschiedlichen Gebieten. Alexander Grosch danke ich für die Unterstützung bei den motorischen Experimenten, Karsten Roetmann für seine Hilfe bei der Einarbeitung in die Kameratechnologie und Alexander Göhmann dafür, dass er mir bei Fragen der technischen Konstruktion immer behilflich war. Für die Anfertigung der mechanischen Teile für die Versuchsaufbauten danke ich Herrn Andreas Sparta.

Einen großen Dank gebührt auch Herrn Dr. Hainer Wackerbarth, der mich in der finalen Phase dieser Arbeit betreute und mich beim Verfassen der Dissertation maßgeblich unterstützte.

Ein herzliches Dankeschön an meine Kollegen Ronja Bäumner, Britta Kreilein, Dr. Sven Groß und Dr. André Selle für die netten abwechslungsreichen Gespräche, die für eine angenehme Atmosphäre sorgten. Dr. Sven Groß, Dr. Selle und Dr. Konstantin Christou danke ich für den Erfahrungsaustausch über die Organisation der Disputationsprüfung.

Zuletzt und als erstes gilt mein besonderer Dank an meine Freundin Annika Linke, die mich zu jeder Zeit moralisch unterstützte und mir immer wieder Kraft und Ansporn lieferte. 



\section{Lebenslauf}

\section{Ralf Müller}

geboren

Nationalität

Tätigkeit

14. Februar 1974 als Sohn von Hermann Müller und Rosa Müller, geb. Harrant, in Alfeld/Leine

deutsch

Doktorand am Laser-Laboratorium Göttingen e.V.

\section{Hochschulausbildung}

seit 2004

Doktorarbeit an der an der Universität Göttingen

2003

Diplomarbeit am Max-Planck-Institut für biophysikalische Chemie, in Göttingen, Thema: „Untersuchung des Rotationsverhaltens von Biomolekülen mit Hilfe von fluoreszenzspektroskopischen Methoden"

$1998-2003$

Diplom-Studiengang Physik

\section{Schulbildung}

1997

Abitur (Leistungskurse Physik und Elektrotechnik)

$1994-1997$

Werner-von-Siemens-Schule in Hildesheim

$1987-1991$

Realschule in Delligsen

\section{Zivildienst}

1997-1998

Zivildienst beim Paritätischen Wohlfahrtsverband in Alfeld/Leine

\section{Zusätzliche Ausbildungen}

$1991-1994$

Ausbildung zum Physiklaboranten bei der Deutschen Spezialglas AG in Grünenplan 